\title{
The Structure of the Star-forming Cluster RCW 38
}

\author{
E. Winston ${ }^{1}$, S. J. Wolk ${ }^{2}$, T. L. Bourke ${ }^{2}$, S.T. Megeath ${ }^{3}$, R. Gutermuth ${ }^{4,5}$, B. Spitzbart ${ }^{2}$ \\ ewinston@rssd.esa.int
}

\begin{abstract}
We present a study of the structure of the high mass star-forming region RCW 38 and the spatial distribution of its young stellar population. Spitzer IRAC photometry $(3-8 \mu \mathrm{m})$ are combined with 2 MASS near-IR data to identify young stellar objects by IR-excess emission from their circumstellar material. Chandra X-ray data are used to identify class III pre-main sequence stars lacking circumstellar material. We identify 624 YSOs: 23 class 0/I and 90 flat spectrum protostars, 437 Class II stars, and 74 Class III stars. We also identify 29 (27 new) O star candidates over the IRAC field. Seventy-two stars exhibit IR-variability, including seven class 0/I and 12 flat spectrum YSOs. A further 177 tentative candidates are identified by their location in the IRAC [3.6] vs. [3.6]-[5.8] cmd. We find strong evidence of subclustering in the region. Three subclusters were identified surrounding the central cluster, with massive and variable stars in each subcluster. The central region shows evidence of distinct spatial distributions of the protostars and pre-main sequence stars. A previously detected IR cluster, DB2001_Obj36, has been established as a subcluster of RCW 38. This suggests that star formation in RCW 38 occurs over a more extended area than previously thought. The gas to dust ratio is examined using the X-ray derived hydrogen column density, $N_{H}$ and the $K$-band extinction, and found to be consistent with the diffuse ISM, in contrast with Serpens \& NGC1333. We posit that the high photoionising flux of massive stars in RCW 38 affects the agglomeration of the dust grains.
\end{abstract}

\footnotetext{
${ }^{1}$ ESA-ESTEC (SRE-SA), Keplerlaan 1, 2201 AZ Noordwijk ZH, The Netherlands.

${ }^{2}$ Harvard Smithsonian Center for Astrophysics, 60 Garden St., Cambridge MA 02138, USA.

${ }^{3}$ Ritter Observatory, Dept. of Physics and Astronomy, University of Toledo, 2801 W. Bancroft Ave., Toledo, OH 43606, USA.

${ }^{4}$ Five Colleges Astronomy Department, Smith College, Northampton, MA 01027

${ }^{5}$ Department of Astronomy, University of Massachusetts, Amherst, MA 01003
} 
Subject headings: infrared: stars — X-rays: stars — stars: pre-main sequence — circumstellar matter

November 20, 2018

\section{Introduction}

The RCW 38 cluster is a region of high mass star formation, lying at a distance of $1.7 \mathrm{kpc}$ from Earth. The region is one of the closest high mass star forming regions known to contain more than a thousand members (Wolk et al. 2006), after clusters such as the Orion Nebula Cluster (Muench et al. 2008), Cep OB3b (Getman et al. 2006), and NGC 2244 (Wang et al. 2008). It was first identified in the $H \alpha$ survey of Rodgers, Campbell, Whiteoak (1960) and the HII survey of Gum (1955). The heart of the RCW 38 cluster is dominated by the O5.5 binary RCW 38 IRS 2 (Frogel \& Persson 1974; DeRose et al. 2009). Yamaguchi et al. (1999) obtained ${ }^{13} \mathrm{CO}$ NANTEN data of a large region surrounding RCW 38, showing two clouds, the eastern one coincident with IRS 2, while the second extends to the west. Previous observations of RCW 38 by DeRose et al. (2009) using VLT/NACO identified 314 members from a total of 336 detections in the $J, H, K_{s}$-bands, in a field 0.5 pc squared surrounding IRS2. RCW 38 has previously been observed with Chandra as reported by Wolk et al. (2006), where 360 X-ray sources from a total of 460 were positively associated with the star forming region. An extensive overview of the previous studies of RCW 38 is to be found in the Handbook of Star Formation article on the cluster (Wolk, Bourke \& Vigil 2008).

Why are observations of young high mass star forming regions important to our understanding of star formation? Current estimates place more than $60 \%$ of star formation in clustered environments (Cartwright \& Whitworth 2004; Megeath et al. 2004). It is therefore important to perform multi-wavelength studies of such regions to understand the influence of the cluster environment in the process of star and planet formation. One fundamental issue is the the spatial structure of clusters and the evolution of that structure. The morphology of the molecular cloud as it undergoes collapse affects the subsequent star formation in the region. By examining the spatial distribution of the young stars in a cluster it is possible to trace the underlying structure of the molecular cloud from which they form. Such studies may differentiate between regions undergoing spherically symmetric collapse and those were the cloud is fragmenting to form distinct subclusters (Feigelson \& Townsley 2008).

Regions of high mass star formation within $\sim 2 \mathrm{kpc}$ have been shown to exhibit varying 
morphologies: For example, Wang et al. (2011) and Wolk et al. (2011) have recently published studies of Trumpler $15 \& 16$ in Carina, a massive star forming complex at a distance of $2.3 \mathrm{kpc}$, and find very different structures. Tr 15 has a compact spherical structure with a continuous envelope of dispersed stars and mass segregation, Tr 16 has seven subclusters and no evidence of mass segregation. The subclusters in $\operatorname{Tr} 16$ are contiguous; they contain massive stars but none are located within $0.2 \mathrm{pc}$ of the cluster center. Both regions have very low circumstellar disk fractions $(<10 \%)$ consistent with ages of 3-10Myr. NGC 2244 in the Rosette Nebula lies at a distance of $1.4 \mathrm{kpc}$ and was recently studied by Wang et al. (2008). It has as an annular morphology and a central cluster of O-stars, with a larger scale spherical structure. There is no evidence of subclustering and a low disk fraction $(\sim 6 \%)$ though its age is estimated at $2 \mathrm{Myr}$. The RCW 108 region, at a distance of $1.3 \mathrm{kpc}$, is a nearby example of triggered star formation (Comerón et al. 2005), with evidence of mass segregation and its YSO population still embedded in its molecular cloud (Wolk et al. 2008). In RCW 38, where the disk fraction is consistent with an age of $1 \mathrm{Myr}$, we will present evidence of four separate subclusters surrounded by a dispersed population, with evidence of mass segregation and possible triggering of a second generation of low mass stars surrounding IRS 2.

Evolution of dust grains in the ISM and within molecular clouds can have important implications for the development of circumstellar disks and hence the formation of planets around young stars. Of specific importance is the study of the effect of massive stars in densely populated star-forming regions, to examine the effects of ionising radiation on the evolution of disks around lower mass stars, and to examine the star formation efficiency where much of the gas and dust are processed by stellar radiation fields. The gas to dust ratio can be examined through the measured dust extinction in the IR and the X-ray calculated hydrogen column density. In previous studies of less massive clusters, the authors found possible evidence for grain growth in Serpens North and NGC 1333 (Winston et al. 2007, 2010). Higher mass regions, such as RCW 108 and $\mathrm{LkH} \alpha 101$ have been found to be similar to the ISM (Wolk et al. 2008, 2010). Wolk et al. (2006) previously found a value of $N_{H}=$ $2 \times 10^{21} A_{V}$ for this relation in RCW 38. The implication that the presence of massive stars in the vicinity impedes grain growth will here be re-examined making use of the improved IRAC evolutionary classification of the YSOs.

Observations with the Spitzer and Chandra Space Telescopes provide the means to identify young stellar objects from the protostellar through the diskless pre-main sequence phase. The IR data further allows for the categorization of the objects into the canonical evolutionary classes (class 0/I, II and III (Lada \& Wilking 1984)). The X-ray data of Wolk et al. (2006) are utilized here in conjunction with the Spitzer IRAC data to identify young diskless members of the cluster. Information on the distribution of star formation sites, the dispersal after formation of the YSOs, and the dynamical state of the complex 
as a whole can be found by examining the number and distribution of the YSOs. Such studies provide a better understanding of the physical processes that govern the collapse and fragmentation of molecular clouds, the effect of massive stars on the evolution of their less massive neighbors, and of the possible future dispersal of the cluster after the natal gas has been dispersed. Clusters also serve as laboratories for the evolution of young stellar objects, and can be used to study the evolution of disks around young stars and the evolution of hot X-ray emitting plasma commonly found around such stars (see for example: Gutermuth et al. (2009); Haisch et al. (2001); Preibisch \& Feigelson (2005); Hernadez et al. (2006); Winston et al. (2007); Wolk et al. (2010)).

In this paper we present the Spitzer IRAC observations of the extended RCW 38 cluster, identifying 624 YSOs in the region. We first present a description of the Spitzer data and its reduction. Second, we discuss the techniques used in the identification of the cluster members, and the evolutionary classification of the young stars. We then discuss the Xray characteristics of the stars as compared to the infrared characteristics, the temporal photometric variability of the young stars, the selection of O-star candidates in the region, and lastly the spatial distribution of the cluster members is presented.

\section{Observations and Data Reduction}

We have obtained observations of the RCW 38 region with the Spitzer InfraRed Array Camera (IRAC; Fazio et al. (2004)) in four wavelength bands: 3.6, 4.5, 5.8, and $8.0 \mu \mathrm{m}$ at four different epochs. Fig. 1 and Fig. 2 show IRAC three band false-color images of the combined field of view of the four epochs and the central core of RCW 38. We also obtain data with at $24 \mu \mathrm{m}$ from the Multiband Imaging Photometer for Spitzer (MIPS; Rieke et al. (2004)) at two separate epochs. This photometry was supplemented by $J, H$ and $K$-band photometry from the 2MASS point source catalogue (Skrutskie et al. 2006). In the following analysis, only data with uncertainties $\leq 0.2$ magnitudes were used. Below, we describe the observations, image reduction and photometry for the Spitzer data.

\subsection{Mid-IR: Spitzer IRAC}

The Spitzer Space Telescope has observed the RCW 38 cluster with the IRAC instrument at four different epochs as part of PID 20127. This observing strategy purposed to fully cover the $\sim 30^{\prime} \times 30^{\prime}$ field while recovering the inner $\sim 15^{\prime} \times 15^{\prime}$ with shorter exposures to overcome the high background of the central core. The first epoch, Epoch1, was taken on 2006-06- 
05 and utilized cluster mode to map the outer field in a spiral pattern with each pointing offset by $\sim 270^{\prime \prime}$, with 12 second frames and 5 dithers per position with the cycling dither turned on. The map ends before the interior region of $\sim 6^{\prime} \times 6^{\prime}$ was observed to avoid the build-up of short-term latents on the image. The second epoch, Epoch 2, was taken directly following Epoch 1 on 2006-06-05 but observed a smaller $\sim 15^{\prime} \times 15^{\prime}$ central field of RCW 38 . An integration time of 2 seconds with 1 dither per position with the cycling dither turned on was used to avoid saturation. The third epoch, Epoch 3, was observed on 2007-02-21 and followed the same spiral pattern over the outer field of $\sim 30^{\prime} \times 30^{\prime}$ surrounding the RCW 38 core, again ending before the core was observed. The last epoch, Epoch 4, directly followed Epoch 3 and was taken on 2007-02-21 and also observed a $\sim 15^{\prime} \times 15^{\prime}$ IRAC overlap region of the RCW 38 core. Table 1 lists the integration times and coverage of the four epochs.

The analysis used the basic calibrated data (BCD) FITS images from the S14.0.0 pipeline of the Spitzer Science Center for Epochs $1 \& 2$ and the S15.3.0 pipeline for Epochs $3 \& 4$. These data were combined into mosaics using WCSmosaic, which also removed cosmic ray and other artifacts. Custom IDL routines, part of the clustergrinder package (Gutermuth et al. 2009) were used to clean the mosaics of bright source artifacts. The pixel scale of the mosaics is $1.2 \%$ pixel. The calibration uncertainty across the IRAC bands is estimated at $0.02 \mathrm{mag}$ (Reach et al. 2005). In addition, there are $\sim 5 \%$ position dependent variations in the calibration of point sources in the flat-fielded BCD data; these have not been corrected for in our data. Furthermore, these systematic uncertainties have not been added into the photometric uncertainties reported in this paper. The noise in a $8 \times 8$ pixel region around the source was calculated to assess the combined instrumental noise, shot noise and noise from the spatially varying extended nebulosity. Point sources were found using Photvis, based on a heavily modified version of the DAOfind program in the IDLPHOT package (Landsman 1989). Point sources with peak values more than $5 \sigma$ above the background were considered candidate detections. After identifying the point sources, aperture photometry was obtained using the aper.pro program in the IDLphot package. An aperture radius of 2 pixels was used, and a sky annulus from 2 to 6 pixels was used to measure the contribution from extended emission in the aperture with $\mathrm{mmm}$.pro, also in the IDLphot package. The zero points for these apertures were: 17.0879, 16.5843, 15.9790, and $15.1945 \mathrm{mag}$ for the 3.6, 4.5, 5.8, $8.0 \mu \mathrm{m}$ bands respectively, in the native BCD image units of $\mathrm{MJy} \mathrm{sr}^{-1}$.

In Epoch 1, there were 4195 detections in the 3.6 $\mu \mathrm{m}$ band with uncertainties less than $0.2 \mathrm{mag}$, and 3346, 1536, 653, in the 4.5, 5.8, and 8.0 $\mathrm{m}$ bands, respectively, with 302 detections in all four IRAC bands and 816 detections in the three shortest IRAC bands. In Epoch 3, there were 4137 detections in the 3.6 $\mu \mathrm{m}$ band, and 3941, 1596, 893, in the 4.5, 5.8, and $8.0 \mu \mathrm{m}$ bands, respectively, with uncertainties less than $0.2 \mathrm{mag}$, with 415 detections in 
all four IRAC bands and 1,039 in the three shortest IRAC bands. In the smaller deeper field Epoch 2, there were 2106 detections in the $3.6 \mu \mathrm{m}$ band with uncertainties less than $0.2 \mathrm{mag}$, and $1605,713,205$, in the 4.5, 5.8, and $8.0 \mu \mathrm{m}$ bands, respectively, with 108 detections in all four IRAC bands and 329 in the three shortest bands. In Epoch 4, there were 1775 detections in the $3.6 \mu \mathrm{m}$ band with uncertainties less than $0.2 \mathrm{mag}$, and $1844,454,299$, in the 4.5, 5.8, and $8.0 \mu \mathrm{m}$ bands, respectively, with 116 detections in all four IRAC bands and 311 in the three shortest bands. The sensitivity of the $8.0 \mu \mathrm{m}$ band is the limiting factor in four band detections: it suffers from lower photospheric fluxes, higher background emission, and the presence of bright, structured nebulosity across much of the image. The arrangement of the detectors ensures that only a fraction of the sources can have 4-band detections in any single epoch (c.f Sec.2.4) The detection threshold in the $3.6 \mu \mathrm{m}$ band was $\sim 17$ magnitudes - just at the hydrogen burning limit for a $1 \mathrm{Myr}$ old star at the distance of RCW 38 ( 16.5 mag at $3.6 \mu \mathrm{m}$ (Baraffe 1998)).

\subsection{MIPS:}

We observed the RCW 38 cluster on two occasions with the Multiband Imaging Photometer for Spitzer (MIPS; Rieke et al. 2004). The observations were taken as part of PID 20127. The first epoch was observed on 2006-03-03 and had an aim point of $8^{h r} 59^{m} 5.78^{s}$, $-47^{d} 30^{m} 43.02^{s}$ (J2000). The second epoch was observed on 2006-06-18 and had an aim point of $8^{h r} 59^{m} 5.49^{s},-47^{d} 30^{m} 41.14^{s}$ (J2000). In both epochs, the medium scan rate was used and the total map sizes were approximately $0.5 \times 0.5$ degrees including the overscan region. The typical integrated exposure time per pixel is about 2.62 seconds at $24 \mu \mathrm{m}$. The MIPS images were processed using the MIPS instrument team Data Analysis Tool, which calibrates the data and applies a distortion correction to each individual exposure before combining into a final mosaic (Gordon et al. 2005). The data were further processed using various median filters to remove saturation effects and column-dependent background structure. The resulting mosaics have a pixel size of $1.25^{\prime \prime}$ at $24 \mu \mathrm{m}$. The $24 \mu \mathrm{m}$ images suffered from saturation of the central core region and strong extended emission over the field; it was not possible to extract reliable photometry for any objects, instead the $24 \mu \mathrm{m}$ mosaic was used to search for deeply embedded protostars surrounding the core (cf. Sec. 5.4).

\subsection{Chandra:}

This paper utilizes the previous Chandra observations of RCW 38, first presented in

Wolk et al. (2006) and now available on the ANCHORS archive (AN archive of Chandra 
Observations of Regions of Star formation 1 ). The observations were reprocessed and an updated source detection procedure was applied for the archive, and this reprocessed dataset is used here. Originally, the data were processed in 2001 through the CXCDS ver.6.12 pipeline. A wavelet based source detection algorithm, originally developed for ROSAT, known as PWDetect (Damiani et al. 1996) was then used to identify sources across the entire ACIS-I array. The array was $2 \times 2$ binned and the threshold significance was set to detect sources between 4 and 5.31 equivalent Gaussian sigmas; the data are searched on scales of 0.5 to $16 "$. With these settings, a false detection rate of $<1 \%$ is expected. A total of 460 sources were found. The 2x2 binning, employed due to memory constraints means the the image is under-sampled with respect to the maximum resolution of ACIS. Hence, some close sources, on-axis, can be conflated. For the new source extraction, a three-pass method was used, with the CIAO 3.4 Wavedetect tool (Freeman et al. 2002). The threepass method searches for sources in images binned by 1,2 and 4 pixels, respectively, using wavelet scales of 2, 4, 8, 16 and 32 pixels on each image. Each image has a size of 2048x2048 pixels. This method was designed to optimally take advantage of additional memory more commonly available in 2007. The source limits were again set to limit false detections to about 1\%, however Wavdetect has more robust error analysis and tests indicated that this was slightly more sensitive than PWDetect at the nominal $1 \%$ false detection probability. The results of the three passes are combined into a single list of 536 sources. This includes 18 sources detected on the S array which was not considered to by Wolk et al. (2006). By comparison the Chandra Source Catalog (Evans et al. 2010) detects 410 sources in the field with a minimum flux threshold of about 10 net counts on axis, which is significantly higher than our equivalent limit. Table 2 lists the identifiers, coordinates and properties of the 536 sources identified in this reprocessed dataset. The table provides the Chandra identifier, source locations, the raw and net number counts (net counts are background subtracted and aperture corrected), plasma temperature $(k T)$, hydrogen column density $\left(N_{H}\right)$, absorbed and unabsorbed X-ray flux $\left(F_{X}\right)$, and flaring statistics.

\subsection{Catalog Merging}

For each epoch of Spitzer data, the photometry of the four IRAC bands were merged with the 2MASS $J, H$, and $K$-band and Chandra X-ray data to form an initial catalogue using custom IDL routines. Sources observed in different wavelength bands, which were located within $1^{\prime \prime}$ of each other were merged in the catalogue; if while comparing two bands, multiple sources in one of the bands satisfied this criteria, the closest was chosen. The

\footnotetext{
${ }^{1}$ http://cxc.harvard.edu/ANCHORS/
} 
Chandra X-ray data were taken from Wolk et al. (2006), where a full description of the data reduction process was presented. The observation, Chandra obsid 2556, had a total exposure time of $97 \mathrm{ks}$, with 536 sources detected in the Chandra field. These catalogues were used in the identification of the YSOs, c.f. Sec.3.

The fields of view (FOVs) at each IRAC epoch, and of the different instruments did not cover the same extent of the cluster, with the IRAC epochs $2 \& 4$ limited to a $15^{\prime} \times 15^{\prime}$ field in the central cluster. The two larger fields, epochs $1 \& 3$, cover a $30^{\prime} \times 30^{\prime} \mathrm{FOV}$ of the cluster, with a $\sim 6^{\prime} \times 6^{\prime}$ gap in coverage to avoid saturation in the cluster core. Furthermore, the IRAC detectors are split into two groups, channels $1 \& 3(3.6 \& 5.8 \mu \mathrm{m})$ and channels 2 $\& 4(4.5 \& 8.0 \mu \mathrm{m})$, whose FOVs are offset from one another by $6.5^{\prime}$. The Chandra field is the smallest overall, covering a region $17^{\prime} \times 17^{\prime}$, centered on IRS 2. The X-ray sample is thus limited to the central region of the cluster, and data are not available for all IR sources in the catalogue. The 2MASS data are not spatially constrained. A final photometric catalogue was then created by matching the sources detected in the four initial catalogues to within 1". This catalogue was used in the variability study, c.f. Sec 5.2 .

\section{Identification of YSOs}

The RCW 38 field contains 26,873 sources with a detection in at least one IR band, at one epoch, across the entire IR field. In the IRAC overlap field, there were 13,950 sources with a detection in at least one IR band. In order to identify the possible young stellar objects (YSOs) in the four epochs, two selection techniques were applied to the data: selection of stars with IR excesses on IR color-color diagrams, and identification of X-ray luminous YSOs by comparison of X-ray sources with IR detections. These methods were applied to each of the four IRAC epochs separately in the IR field, after which, the four catalogues of candidate YSOs were merged into one master list of candidate members of RCW 38. This list was then trimmed of those candidates not appearing in the IRAC overlap field of at least one epoch.

In each epoch, the field was limited to that region observed by all four IRAC bands: the overlap IRAC field. By examining this restricted field, we insure that each individual source has the possibility of being detected at all four IRAC wavelengths. Each of the four IRAC epochs was examined individually from the others, but each was 2 combined with the same X-ray and near-IR observations. 


\subsection{Infrared Excess Emission}

Young stellar objects can be identified by their excess emission at IR wavelengths. This emission arises from reprocessed stellar radiation in the dusty material of their natal envelopes or circumstellar disks. The infrared identification of YSOs is carried out by identifying sources that possess colors indicative of IR excess and distinguishing them from reddened and/or cool stars (Megeath et al. 2004; Allen et al. 2004; Gutermuth et al. 2004). The main limitation of this method is the contamination from extragalactic sources such as PAH-rich star-forming galaxies and AGN, and galactic sources such as AGB stars; both of these groups have colors similar to young stellar objects. Four color-color diagrams were used to determine the color excess of the sources: two IRAC diagrams: [3.6] - [4.5] vs. [5.8] - [8.0] and [3.6] - [4.5] vs. [4.5] - [5.8], and two IRAC-2MASS diagrams: $J-H$ vs. $H-[4.5]$ and $H-K$ vs. $K-[4.5]$. Fig. 3 shows the four diagrams for each IRAC epoch (Ep.1-4; note that the 2MASS photometry is the same throughout). In the following analysis we required photometry to have uncertainties $<0.2$ mags in all bands used for a particular color-color diagram. The numbers of sources for each diagram which satisfy this criteria are, respectively, in Epoch 1: 302, 816, 2748, 3007; in Epoch 2: 108, 329, 1084, 1342; in Epoch 3: 415, 1039, 3068, 3478; in Epoch 4: 116, 311, 1195, 1534.

\subsubsection{IRAC Color-Color Diagram}

The main effort involved in identifying bone fide IR excess sources is distinguishing between reddened or cool stars and those with excess emission arising from circumstellar material. A reddening law in the IRAC bands was determined by Flaherty et al. (2007), which shows the [5.8] - [8.0] color to be particularly insensitive to reddening and stellar temperature, and is thus a reliable measure of excess emission due to dust. Sources with a color $>1 \sigma$ beyond [5.8] $-[8.0]>0.2 \mathrm{mag}$ are likely to possess excesses and not to be reddened or cool stars. Extragalactic sources such as PAH rich star-forming galaxies and AGN will also satisfy this criteria (Stern et al. 1994). Sources with a color more than $1 \sigma$ below [3.6] $-[4.5]<0.1$ were considered galaxies and were removed. AGB stars exhibit colors similar to reddened or cool stars over the IRAC bands (Blum et al. 2006) and are filtered out with the field stars.

Gutermuth et al. (2009) have recently developed a method for substantially reducing extragalactic contamination built on the Bootes Shallow Survey data (Eisenhardt et al. 2004)

and the Spitzer cores to disks legacy program methods (Jorgensen et al. 2006; Harvey et al. 2006). The galaxies are eliminated either by their colors, which unlike YSOs are often dominated by PAH features in the 5.8 and $8.0 \mu \mathrm{m}$ bands, or by their faintness. It should be 
noted that very faint or embedded protostellar sources may be erroneously filtered by this method. A total of 69 candidate YSOs were selected from the IRAC color-color diagram in Epoch 1, with 56 in Epoch 2, 119 in Epoch 3, and 54 in Epoch 4. Of these 17 were identified as contaminating sources and removed from the Epoch 1 catalogue, 8 from Epoch 2, 11 from Epoch 3, and 9 from Epoch 4.

\subsubsection{IRAC-2MASS Color-Color Diagrams}

The shorter wavelength 3.6 and $4.5 \mu \mathrm{m}$ IRAC bands are far more sensitive to stellar photospheres than the longer wavelength 5.8 and $8.0 \mu \mathrm{m}$ bands which suffer from higher backgrounds; e.g. in Epoch 1 where the shorter wavelength bands have 4195 and 3346 detections with $\sigma \leq 0.2$, respectively, the longer wavelength bands have 1536 and 653 detections. Hence many detections cannot be characterized on the IRAC color-color diagram. For this reason, it is important to develop methods to identify sources with infrared excesses that rely only on the shorter wavelength bands.

We combine the near-IR data from the 2MASS point source catalog with the $4.5 \mu \mathrm{m}$ band photometry (Skrutskie et al. 2006). This provides the ability to detect dust around objects which are too faint for detection in the 5.8 and $8.0 \mu \mathrm{m}$ bands. In particular, we concentrate on the $J-H$ vs. $H-[4.5]$ diagram and the $H-K$ vs $K-$ [4.5] diagram (Winston et al. 2007). These diagrams take advantage of the sensitivity of Spitzer in the $4.5 \mu \mathrm{m}$ band and the stronger infrared excess emission at $4.5 \mu \mathrm{m}$ compared to that at shorter wavelength (Gutermuth 2005). For highly reddened sources which are not detected in the $J$-band, the $H-K$ vs. $K-[4.5]$ diagram can be used. It should be noted that the IRAC $4.5 \mu \mathrm{m}$ data can detect sources too faint or reddened to have been detected by 2MASS. AGN are typically fainter than stellar YSOs found in star-forming regions; all candidates with $3.6 \mu \mathrm{m}$ magnitudes fainter than 16 mag that lacked the required bands to be placed

on the Gutermuth et al. (2009) diagrams were removed. Utilizing the $J-H$ vs. $H-[4.5]$ and $H-K$ vs. $K-[4.5]$ diagrams, in Epoch 1, 109 and 120 sources were selected as having an IR excess, 72 and 99 in Epoch 2, 160 and 180 in Epoch 3, and 86 and 118 in Epoch 4, respectively.

\subsubsection{IRAC 3-Band Color-Color Diagram}

Due to the limiting factor of requiring an $8 \mu \mathrm{m}$ detection to place a source on the IRAC CCD, we also utilized the IRAC 3-band CCD [3.6]-[4.5] vs. [4.5]-[5.8] to identify sources with 
excess emission but with photometry only in the $3.6-5.8 \mu \mathrm{m}$ bands. The criteria for young excess sources on this diagram are presented in Gutermuth et al. (2009). Contamination from shocked emission and PAH contamination is dealt with by the selection criteria, however some AGN interlopers may remain. Sources with $3.6 \mu \mathrm{m}$ magnitudes fainter than $16 \mathrm{mag}$ were removed as possible AGN. In Epoch 1, 101 sources were identified as having an excess using this method, of which 24 were not previously known from the other IR diagrams. In Epoch 2, 145 sources were identified, of which 27 were new. In both Epochs 3 \& 4, 101 objects were identified, with 26 and 25 new sources, respectively. Combining the four epochs, a total of 33 additional YSOs were identified using this method.

In addition to this method, the color-magnitude diagram of [3.6] vs. [3.6-5.8] was employed to identify possible cluster members not identified on the previous diagrams due to weak or no excess emission from the disk or a lack of photometry at $8.0 \mu \mathrm{m}$. Figure 4 shows the [3.6] vs. [3.6-5.8] cmd, with field stars shown as black dots, the previously identified YSOs as grey circles, and the newly selected candidates as dark grey stars. Those sources with $8.0 \leq m_{3.6} \leq 14.0$ and a color more than $1 \sigma$ greater than $[3.6-5.8]>0.4$ were selected. This region delimited the color-magnitude space of the previously identified YSOs on this diagram. We identified a further 177 candidates with the appropriate colors. Given that contamination cannot be identified using the contamination removal methods outlined in Gutermuth et al. (2009), we consider these to be tentative candidate members only and do not include them in the following discussions, c.f. Sec. 5. The coordinates and photometry of the 177 candidates are presented in Table 11 .

\subsubsection{Completeness}

The RCW 38 cluster lies at a distance of $1.7 \mathrm{kpc}$ and exhibits a highly variable background across the extent of the cluster. Completeness limits for the Spitzer photometry were assessed using a method of inserting artificial stars into the mosaics and then employing our detection algorithms to identify them. Estimates for the $90 \%$ completion limits for all epochs in each of the IRAC bands were 13.5, 13.0, 12.0, 10.5 mag, respectively. These limits correspond to an approximate stellar mass of $0.5 M_{\odot}$ at $3.6 \mu \mathrm{m}$ and an age of $1 \mathrm{Myr}$ (Baraffe 1998). Fig.5 shows the magnitude histograms of the $3.6 \mu \mathrm{m}$ photometry at each of the four epochs; all detected sources with uncertainties $\leq 0.2$ are shown by the upper black curve. The red histograms show the sources with detections in bands required to place them on at least one of the IR excess diagrams. The sample of identified YSOs is shown by the green histogram. The blue histogram shows the subsample of identified YSOs that possess an X-ray detection. 
The Hydrogen-burning limit, at $1.7 \mathrm{kpc}$, is $\sim 17 \mathrm{mag}$ at $3.6 \mu \mathrm{m}$ and an age of $1 \mathrm{Myr}$ (Baraffe 1998). Though the photometry is sensitive to this magnitude, at this distance source crowding and the bright, highly varying background become serious issues and, as can be seen from the histogram of identified YSOs, our identification threshold is closer to $15 \mathrm{mag}$ at $3.6 \mu \mathrm{m}$, or $\sim 0.15 M_{\odot}$ at $1 \mathrm{Myr}$ (Baraffe 1998). When considering objects that can be placed on the CCDs we are limited to $\sim 13.5 \mathrm{mag}$ at $3.6 \mu \mathrm{m}$ (or $0.5 M_{\odot}$ ). The ratio of the number of sources with $m_{3.6} \leq 13.5 \mathrm{mag}$ that can be placed on the color-color diagrams to the total number of sources with $m_{3.6} \leq 13.5 \mathrm{mag}$ (and correcting this number for completeness by dividing by the fraction of artificial stars recovered in each magnitude bin) is $1778 / 1883$ or $94 \%$ for Epoch 1, 993/1094 or 90\% for Epoch 2, 1822/1966 or 92\% for Epoch 3, 867/975 or $89 \%$ for Epoch 4. These fractions represent upper limits on the completeness of the sample, as the number of contaminating field stars is rising with increasing magnitude, while the number of YSOs does not show an equivalent rise.

The DeRose et al. (2009) study demonstrates the loss of detections towards the RCW 38 nebula in the IRAC data. DeRose et al. (2009) identify 483 sources with uncertainty $<0.2$ in at least one near-IR band in the central 0.5 pc surrounding IRS 2; in comparison, we detect 31 objects in at least one IRAC band with uncertainty $<0.2$ in the same region. The measured stellar surface density in DeRose et al. (2009) is ten times that found with Spitzer due to the higher angular resolution available with the VLT and the reduced confusion with nebular emission achieved toward the RCW 38 nebula in the near-IR.

A more conservative estimate for the $90 \%$ completeness of the pre-main sequence membership of the cluster (those with IR-excess emission) can be made from the green histograms of Fig. [5 as $12 \mathrm{mag}$ at $3.6 \mu \mathrm{m}$, or $\sim 2 M_{\odot}$ at $1 \mathrm{Myr}$ (Siess et al. 2000). This estimate does not account for extinction, which will move completeness to even higher masses. Further, completeness varies across the field due to the varying background emission, being particularly low in the central region surrounding IRS 2. Since the fraction of sources with IR-excess varies with mass and age (Lada 1987; Hernadez et al. 2006), the completeness with respect to all pre-main sequence stars cannot be estimated with the available data.

\subsection{X-ray Luminous Objects}

Young stellar objects possess elevated levels of X-ray emission, with luminosities, $L_{X}$, of $\frac{L_{X b o l}}{L_{X}} \sim 10^{3.5}$ that can be used to distinguish them from foreground or background field stars (Feigelson \& Montmerle 1999; Feigelson \& Kriss 1981). We utilize this property to identify YSOs that do not have emission from a dusty disk (evolutionary class III) and would otherwise be indistinguishable from field stars. Protostars (class 0/I) and pre-main 
sequence stars with disks (class II) with elevated X-ray emission may also be detected. Wolk et al. (2006) observed the core of RCW 38 in a 96 ks observation. The Chandra image of RCW 38 covered a $17^{\prime} \times 17^{\prime}$ field of view centered on the cluster core and IRS2, thus $\mathrm{X}$-ray data is not available over the entire spatial extent of the sources in our catalog. The 2006 catalogue contained 460 detections of which 294 were determined from their X-ray detection and NACO near-IR photometry (DeRose et al. 2009) to be likely young members of the cluster. In this paper we make use of the reprocessed and updated catalogue, available on the ANCHORS online archive (AN archive of Chandra Observations of Regions of Star formation 2), to compare to the IR sources to identify likely cluster members.

There were $536 \mathrm{X}$-ray detections in the catalogue, a mixture of YSOs, AGN, and dMe stars. For each epoch, the coordinates of the X-ray sources were matched to the nearest IR detection to within a radius of $1^{\prime \prime}$. In Epoch 1, 175 X-ray detections were identified with IR sources, 203 in Epoch 2, 190 in Epoch 3, and 198 in Epoch 4. In total, 226 X-ray sources were identified with IR sources. This number is less than the 294 X-ray detections matched in the Wolk et al. (2006) study since the resolution of the Spitzer data is much lower than Chandra in the central $5^{\prime}$ where $\sim 50 \%$ of the X-ray sources are located. The lower limit of the X-ray luminosity detectable in RCW 38 with Chandra can be estimated by comparison with the COUP data, see Feigelson et al. (2005), as $\log _{10} L_{X} \approx 30.1 \mathrm{ergs} \mathrm{s}^{-1}$, assuming a distance of $1.7 \mathrm{kpc}$ to RCW 38, an exposure time of $97 \mathrm{ks}$, and a median value of $\log _{10}\left(N_{H}\right)$ of 22.3. We match the X-ray sources with IR counterparts to reduce the contamination from AGN; an X-ray source with no IR counterpart is assumed to be AGN contamination.

Of those sources identified in X-rays, 97 were previously identified from the IR excess diagrams in Epoch 1, thus 78 YSOs were identified using X-rays. In Epoch 2, 136 were identified in the IR and 67 were X-ray identifications. In Epoch 3, 110 were previously known, with 80 X-ray identifications. In Epoch 4, 131 were identified as excess sources, with 67 detected in X-rays. In total, 74 YSOs were identified through their elevated X-ray emission. Of these, 41 had previously been identified in the Wolk et al. (2006) study, so that 33 are newly identified as members in this work. It should be noted that the ACIS field only partially covers the region, and thus the X-ray detection fractions are only representative of the central region and not the outer distributed YSO population. Wider scale X-ray studies of region would allow us to examine the X-ray properties and dispersion of Class III objects in the region. The completeness of the X-ray data can be assessed from Fig. 5 ; it should be noted that only $67 \%$ of the YSOs from class 0/I to class II lie in the Chandra field of view, thus the completeness of the X-ray data is limited partially by the smaller field. Considering

\footnotetext{
${ }^{2}$ http://cxc.harvard.edu/ANCHORS/
} 
only those young stars located in the Chandra field of view, and those YSOs with $m_{3.6}<12$, $55 \%$ of this sample are detected in X-rays. This percentage remains approximately constant over the range of $3.6 \mu \mathrm{m}$ magnitudes from $8<m_{3.6}<12$. Hence, we estimate the X-ray YSO detection completeness to be $55 \%$ for objects with $m_{3.6}<12$.

\subsection{Summary of Identified Sources}

The four epochs of data were combined, yielding a total of 679 candidate members of the cluster over the IRAC overlap field. Of these, 32 were found to be contaminants: AGN or PAH-rich galaxies, leaving 647 in the IRAC fields. The numbers of YSOs identified in each of epochs 1-4 were: $369,358,504,377$. On trimming this catalog to include only those sources in the IRAC overlap field of one or more epochs, the total number of cluster members was found to be 624 . Of these, 226 were detected in X-rays by Chandra, including 74 that identified as YSOs by their elevated Chandra X-ray emission. The membership of RCW 38 is estimated to be $90 \%$ complete to $12 \mathrm{mag}$ at $3.6 \mu \mathrm{m}$, or $\sim 2 M_{\odot}$ at $1 \mathrm{Myr}$ (Siess et al. 2000). As will be shown, $\sim 40 \%$ of objects in each evolutionary class are detected in X-rays, thus we are possibly missing $\sim 200$ class III members in the ACIS-field. We also identified a further 177 candidate cluster members, which would bring the total membership identified here to 801 .

By scaling the Harvey et al. (2006) results to our $0.2 \mathrm{deg}^{2}$ field, we estimate that there is of order $1 \mathrm{AGB}$ contaminant in our catalogue. We estimate that 1 or less of the class III sources may be a dMe contaminant.

\section{Evolutionary Classification}

The evolutionary classification of the sources was carried out by first noting their locations on the IRAC and IRAC-2MASS color-color diagrams and assigning each source a preliminary class (Winston et al. (2007); Megeath et al. (2004); Allen et al. (2004); Gutermuth et al. (2009), see Fig. 6). Typically, the IRAC color-color diagram was used for the initial assignment of the evolutionary class. This was followed by the construction of SEDs for all sources; where possible the dereddened SED was also constructed. For each SED, a slope $\alpha_{\text {irac }}=$ $d \log \left(\lambda F_{\lambda}\right) / d \log (\lambda)$ was calculated. The conversion from magnitudes to fluxes in $\mathrm{W} \mathrm{cm}^{-2} \mathrm{~s}^{-1}$ used the following zero fluxes for the $J, H, K s$, [3.6], [4.5], [5.8], and [8] bands respectively: $3.13 \times 10^{-13}, 1.13 \times 10^{-13}, 4.28 \times 10^{-14}, 6.57 \times 10^{-15}, 2.65 \times 10^{-15}, 1.03 \times 10^{-15}, 3.02 \times 10^{-16}$ (Skrutskie et al. 2006; Fazio et al. 2004). The slope was calculated by a least-squares fit over 
the available IRAC bands. The 2MASS bands are not included in the fit as they trace the stellar photosphere and tend to bias the fit towards shallower slopes, and thus an earlier evolutionary class. The initial classification from the CCDs is then refined by utilizing $\alpha_{\text {irac }}$, and ${ }_{\text {dered }} \alpha_{\text {irac }}$ where available, to obtain a more precise classification.

The merged master list of YSOs retained the classification assigned in each epoch to those sources detected in multiple epochs. These classes were compared and found to agree in $485 / 624$ cases $(78 \%)$. In those were it did not, 139 cases $(22 \%)$, the class with the most IRAC detections was favored. In 130/139 cases (94\%) the difference in class was between two neighboring evolutionary classes: 13 class 0/I / flat spectrum, 49 flat spectrum / class II, 68 class II / class III. In the case of the class II and class III groups, longer wavelength observations would enable categorization of these objects as potential transition or anaemic disks. Of the remaining nine objects, six were assigned evolutionary classes between class $0 / \mathrm{I}$ and class II, and the remaining three between flat spectrum / class II / class III. The latter three objects were checked and found to be lacking bands in particular epochs, resulting in spurious classifications.

In Fig. 7 the dereddened IRAC slopes, dered $\alpha_{\text {irac }}$, of the YSOs detected at each epoch are shown. The open histograms represent the YSOs, the filled histograms show the Xray selected sample at each epoch, and the lower black histograms indicate the variable sources at each epoch. The median slopes of each epoch are similar; $-1.27 \pm 0.73$ (Epoch $1 ;-1.21 \pm 0.73,-1.36 \pm 0.93,-1.29 \pm 0.88$, for Ep.2, 3, \& 4, respectively). There is no indication of a difference in the median slope (and by extension evolutionary class) between the central core and the outer regions of the cluster. The X-ray sample have median slopes of $-2.19 \pm 0.90$ (Epoch $1 ;-2.12 \pm 0.96,-2.33 \pm 0.94,-2.24 \pm 1.01$ for Ep. 2, 3, \& 4, respectively.), these are biased by the higher fraction of class IIIs included in this sample. The variable sources, which are discussed in Sec. 5.2, have median slopes of $-0.45 \pm 0.99$ (Epoch $1 ;-0.59 \pm 1.05,-0.35 \pm 0.99,-0.29 \pm 0.99$ for Ep. 2, 3, \& 4, respectively). The higher median value of the variables' slopes may be partially due to the smaller sample size, and is indicative of the younger nature of this YSO subsample.

A list of the coordinates and identifiers of the 624 sources identified as young stellar objects in RCW 38 are given in Table 3 to 6. Table 3 lists the coordinates, Spitzer ID, Chandra ID, evolutionary class, and variability indicator of the class 0/I protostars. Table 4 list similar information for the flat spectrum sources, Table 5 the details of the class II objects, and Table 6 the information for the class III members. Similarly, Table 7 to 10 lists the 2MASS and IRAC photometry for the identified YSOs. These tables include measures of the apparent slope of the Spectral Energy Distributions (SEDs) and the extinction at $K_{s}$-band, $A_{K}$. 


\section{Discussion}

There were 26,873 photometric sources detected in at least one IR band across all fields and epochs, of these 13,950 detections were located in the IRAC 4 band overlap region in at least one epoch. Using IR excess emission and X-ray detections, we have identified 624 young stellar objects in the IRAC four band overlap field of view. Of the 624 YSOs, 226 were detected in X-rays with Chandra. The YSOs were of the following evolutionary classes: 23 class 0/I, 90 flat spectrum, 437 class II, and 74 class III. Of the YSOs, 9 class I, 18 flat spectrum, 125 class II, and 74 class III were also identified in the Chandra X-ray observation.

In the following discussion we utilize this sample to address five specific topics. First, we compare the X-ray and IR properties of the YSOs detected with Chandra. Second, we compare the four epochs to determine if their properties vary and to identify variable members of the cluster. Third, we comment on the identification of 28 OB star candidates in the region. Fourth, deeply embedded objects identified in the MIPS observations are briefly discussed. Lastly, we examine the spatial distribution of the cluster YSOs as a function of their evolutionary class, variability and massive star content, and then discuss the implications for the underlying cluster structure.

\subsection{X-ray Characteristics}

In recent years numerous studies have been undertaken to investigate the emission properties of pre-main sequence stars and protostars in the higher energy X-ray region of the spectrum (Wolk et al. 2006; Getman et al. 2005; Winston et al. 2010). In developed, hydrogen burning stars, X-ray activity arises from magnetic fields generated as a result of shear between the core radiative zone and the outer convective zone. In young stars, X-ray emission is thought to arise from mechanisms such as: magnetic disk-locking between the star and disk (Havashi et al. 1996; Isobe et al. 2003; Romanova et al. 2004), accretion onto the star (Kastner et al. 2002; Favata et al. 2003, 2005), or alternative dynamo models for coronal emission (Küker \& Rüdiger 1999; Giampapa et al. 1996). The elevated levels of X-ray emission in PMS stars has been shown to decrease with stellar age (Feigelson et al. 2005), as is observed on the main sequence.

Of the 433 YSOs located within the Chandra field, 226 (52\%) had confirmed X-ray detections. Nine of these were class $0 / \mathrm{I}$ protostars $(9 / 22$ or $41 \%$ of the class $0 / \mathrm{I}$ in the Chandra fov), eighteen were flat spectrum (18/58 or $31 \%$ of the flat spectrum sources), 125 were class II detections (125/279 or $45 \%)$. The remaining 74 sources did not exhibit excess emission and were identified as class III members of the cluster solely on their X-ray 
detection; hence $74 / 624$ or $12 \%$ of the members were identified solely by Chandra. To within $1 \sigma$ there is no apparent trend in rate of detection with evolutionary class, with a $\sim 40 \%$ detection rate for all classes. This detection rate is likely a function of the sensitivity of the Chandra observations. Since we do not possess an independent method of identifying class III young stars we cannot establish the fraction of class III sources detected in Xrays. As we shall subsequently argue the X-ray properties of class II and III objects appear to be similar, we therefore assume that the fraction of class III objects is also similar to that of the class II. Here we neglect any suppression of X-ray activity in class II stars due to accretion (Telleschi et. al. 2007). We thus estimate the total number of class III members to be $(74 / 44.8 \pm 4 \%$ [percentage of CII detected in X-rays]) $60.5 \%$ [correction for Chandra coverage] or $272_{-23}^{+26}$, assuming a disk fraction of $67 \%$ (see following discussion), for an estimated cluster membership of $823_{-23}^{+26}$ objects with $\operatorname{mag}_{3.6 \mu m}>12$.

Of the 226 sources identified as young stars, 138 were previously identified as young members in the Wolk et al. (2006) study. The remaining 88 consisted of 43 which were identified as YSOs by comparison to the IR catalogues in this study and 45 which were new $\mathrm{X}$-ray detections included in this catalogue. Of the 74 class III sources, 41 were previously identified as members by Wolk et al. (2006), the remaining 33 class IIIs are newly identified in this study by their X-ray emission and IR photometry.

The disk fraction of the YSOs, the ratio of the number of X-ray detected disk-bearing pre-main sequence stars (class II) to the total number of X-ray detected pre-main sequence stars (class II \& III) was found to be $125 /(125+74)=63 \pm 6 \%$. On including the Xray detected protostellar sources (class $0 / \mathrm{I} \&$ flat spectrum) this fraction rises to $(9+18+$ $125) /(9+18+125+74)=67 \pm 5 \%$. The disk fractions are calculated based on the assumption of completeness to $12 \mathrm{mag}$ at $3.6 \mu \mathrm{m}$, or $2 M_{\odot}$, and similarity in the detection rates of class II and III sources. These fractions are consistent with a cluster age of $\sim 1$ Myrs (Hernadez et al. 2007). Both are significantly higher than the $29 \pm 3 \%$ found by DeRose et al. (2009) using VLT/NACO in a $\sim 0.5$ pc area surrounding IRS 2. By considering only those of our sources falling in the same field ( 2 class II and 3 class III) we find a disk fraction, $2 / 2+3=40 \pm 28 \%$. Expanding to a 1 pc from the IRS 2 binary, the disk fraction becomes $17 / 17+7=71 \pm 17 \%$, which is comparable to the region as a whole. These results suggest that proximity to the massive IRS 2 stars may have resulted an increased rate of disk processing in the surrounding lower mass YSOs.

There are no discernible trends in $N_{H}$ against $k T$ with evolutionary class, the class II and III objects are similar. The class $0 / \mathrm{I}$ and flat spectrum sources have marginally higher column densities; this would be indicative of their surrounding infalling natal envelopes. The $\mathrm{X}$-ray luminosity of pre-main sequence stars is known to vary with bolometric luminosity 
(Casanova et al. 1995). As was previously found by the authors for Serpens and NGC 1333 (Winston et al. 2007, 2010) and by Hernadez et al. (2007) in $\sigma$ Ori, when using the $J$-band as a proxy for the bolometric luminosity in RCW 38 there is no difference in the dependence of $L_{X}$ on $L_{b o l}$ between class II and III. Fig. 8(right) shows the X-ray luminosity (at an assumed distance of $1.7 \mathrm{kpc}$ ) plotted against the dereddened $J$-band magnitude. A linear fit to the 16 class II sources provides $\log \left(L_{X}\right)=-0.338 \pm 0.027\left(J_{\text {dered }}\right)+35.39 \pm 0.31$, while the fit to eight class II shows $\log \left(L_{X}\right)=-0.331 \pm 0.042\left(J_{\text {dered }}\right)+35.32 \pm 0.52$. This reinforces the suggestion that the mechanisms of X-ray generation are similar for both class II and III and is the basis for our estimate of the class III population. On the left of Fig. 8 the hydrogen column density is plotted against the extinction measured at $K_{s}$-band, $A_{K}$. Only those detections with X-ray count in excess of 100 were considered when examining emission properties to ensure reliable estimates, though in Fig. 8(left) the open symbols also show sources with $>50$ counts.

While this work was not explicitly designed to test gas absorption, $N_{H}$, versus dust extinction, $A_{V} \approx 10 \times A_{K}$, the X-ray data support the use of a simple model of an absorbed thermal plasma to measure $N_{H}$ to within 30\% (Wabs*APEC; Morrison \& McCammon (1983); Smith et al. (2001)). We can then examine the data for any trends in the $N_{H} / A_{K}$ ratio. Historical measurements of $N_{H} / A_{V}$ range from approximately $2.2 \times 10^{21} \mathrm{~cm}^{-2}$ (Ryter 1996) derived from O-star absorption, to roughly $1.6 \times 10^{21} \mathrm{~cm}^{-2}$ (Vuong et al. 2003) derived from a well behaved sample of PMS stars in the $\rho$ Ophichus cluster. Previously, in the lower mass clusters Serpens and NGC 1333, the authors reported on a decreased $N_{H}$ to $A_{K}$ ratio, of $\sim 0.6 \times 10^{22}$ (Winston et al. 2007, 2010), than that quoted for the diffuse interstellar medium in Vuong et al. (2003) of $1.6 \times 10^{22}$. This is thought to be due to the agglomeration of grains in cold dense clouds. However, in RCW 38 the sources are found to fit very well to the ratio of $1.6 \times 10^{22}$, consistent with the Vuong et al. (2003) value for the local ISM and nearby molecular clouds, and with the previous measurement for the region of $2.0 \times 10^{22}$ (Wolk et al. 2006). The class II have a median ratio of $N_{H}=1.66 \pm 0.85 \times 10^{22} A_{K}$ from sixteen sources, while the class III have a median ratio of $N_{H}=1.52 \pm 0.49 \times 10^{22} A_{K}$ from eight objects. The difference may arise from the much higher mass of the RCW 38 cluster. Smith et al. (1999) found that the gas to dust mass ratio towards IRS 1 was $10^{4}: 1$, indicating dust depletion with respect to the ISM. The more active stars may efficiently irradiate the dust in the intercluster medium preventing grain growth or aggregation of dust grains, which would be more likely in the colder medium of the less massive clusters (Cardelli et al. 1989; Stepnik et al. 2003). A larger scale survey of the region, including the outer subclusters, would allow us to ascertain if this difference is more dependent on nascent environment or on the presence of $\mathrm{OB}$ stars in the vicinity. 


\subsection{Variability}

Young stars vary photometrically over time. One of the first criteria, in fact, by which young $\mathrm{T}$ Tauri stars were identified was their photometric variability. This variability occurs across all wavelengths from the UV through optical to IR and into the high energy X-ray regime. The causes are manifold; XU Ori / FU Ori flaring, stellar spots, stellar pulsation, and in the IR, heating of the circumstellar disk (Carpenter et al. 2001). In RCW 38 we have four epochs of observations to search for known YSOs exhibiting variability and for young diskless class III members not detected in X-rays. Given the different regions and exposure times of the outer field and central pointings and the consecutive observations of epochs 1 \& 3 and $2 \& 4$, only the variance of sources between Epochs $1 \& 2$ and $3 \& 4$ were examined. Further, only the IRAC 4 band overlap subsample was considered. Thus we are searching for variability on time scales of $\sim 1$ year. A variable source was defined as one having a difference in magnitude in one or more IRAC bands of $>3 \sigma$ above the combined uncertainties.

Of the 13950 sources in our sample, we identified 72 candidate variable stars. Fig. 9 shows the variation of the 72 variable stars at each of the four IRAC bandpasses for the two timeframes considered. Forty-eight of these were previously identified as YSOs due to excess emission; variability is noted in the final columns of Tables 3.5. The remaining 24 objects were not otherwise found to be YSOs; from an examination of their SEDs it was found that two exhibit excess emission characteristic of circumstellar disks but were not detected in enough bands to be placed on the color-color diagrams, while the other 22 are candidates to be diskless class III young stars. The coordinates, identifiers and photometry of these twenty-four sources are given in Table 12. Of the 24 objects, nine were found to also be O-star candidates and are marked as such in Table 12. Of those 48 previously identified YSOs found to be variable, seven were class 0/I (three X-ray sources), twelve flat spectrum (4 X-ray), and twenty-nine were class II (7 X-ray). No known class III were identified as variable. These numbers represent fractions of variables per class of $30 \pm 11 \%$ for class I, $14 \pm 4 \%$ for flat spectrum, and $7 \pm 1 \%$ for class II. The variability fractions for the X-ray detected sample of YSOs was consistent with that of the non-X-ray detected sample. The protostellar objects have a combined variability fraction of $18 \pm 4 \%$ compared to the $7 \pm 1 \%$ for the disk-bearing class IIs. Such high variability is consistent with larger and/or more frequent accretion events during the protostellar phase.

A study of the properties of these variable sources found that they were evenly spatially distributed across the IRAC overlap field; this is not unexpected as variability is a universal property of young stars. The variables exhibit a similar range of extinctions as the YSOs not found to be varying and there was no trend found between the extinction at $K$-band and the extent of the variance. There was no statistically significant difference in detection 
of variance in X-ray luminous objects or in the X-ray luminosity of the variable members. There is some indication that the percentage of variables decreases with evolutionary class, with $\sim 20 \%$ of the protostellar YSOs varying compared with $\sim 7 \%$ of the class II. None of the previously detected class III sources were found to vary. The extent of variance also appears to show some decrease with evolutionary class: the mean variance of the class 0/I was $0.43 \pm 0.31$, that of the flat spectrum $0.26 \pm 0.16$, the class II $0.27 \pm 0.17$, and those variables showing no infrared excess emission $0.12 \pm 0.12$. The trend is not strongly significant but is consistent with a decrease in the scale of accretion events in the later evolutionary stages.

\subsection{Candidate O Stars}

RCW 38 is one of the nearest massive star forming regions with an estimated membership of more than 1000 members. Previously, Wolk et al. (2006) identified 31 candidate OB stars in a $5 \mathrm{pc}^{2}$ region centered on (and including) IRS2 using an absolute $K$-band magnitude $\geq-0.35$, for a $2.7 M_{\odot}$ at $0.5 \mathrm{Myr}$ (Siess et al. 2000). Twenty were located in the central $\sim 1$ pc. Following Knodlseder (2000) we use the $K$ v. $J-K$ color-magnitude diagram to identify the massive stars in the subsample of objects within the IRAC 4-band overlap region. We identify 29 candidate $\mathrm{O}$ stars and a total of 604 OB star candidates. The coordinates and photometry of the 29 O star candidate sources are given in Table 13. Of the 29 objects, nine were found to overlap with the variable sources and are marked as such in Table 13, An off-field region was subtracted from the cluster to account for field star contamination in the cluster; subtracting the off-field sources yielded an estimate of $26 \mathrm{O}$ stars in the RCW 38 region; thus we estimate that $\sim 3$ of our 29 objects may be contaminants. Two of the objects, 158 and 244, are identified as YSOs and are assigned the evolutionary class II, they are X-ray detected and were also previously identified as candidates by Wolk et al. (2006) as 112 and 396, respectively. The small overlap (2 of 31) between the two lists arises from two causes. Firstly, the smaller field of view of the Wolk et al. (2006) VLT/NACO observations and their higher sensitivity in the cluster centre, where we require a detection in at least one of the IRAC bandpasses. Secondly, the Wolk et al. (2006) study searches for OB star candidates with $M_{K} \leq-0.35$, while here $M_{K} \leq-3.65$. Of the remaining 31 sources, a further nineteen are classified as YSOs, and two more had IRAC detections in too few bands to classify. Seven were not detected as point sources with IRAC. IRS 2, the known O5.5 binary, is not resolved in the IRAC mosaics and does not appear in the candidate list.

Previously, the total population of RCW 38 was estimated as being of order one thousand members. An estimate of the total membership of the RCW 38 region has been presented in Sec. 5.1 as $823_{-23}^{+26}$ objects with $\operatorname{mag}_{3.6 \mu m}>12$. In comparison, Wolk et al. (2011) estimate 
the total population of Trumpler 16, which contains a similar number of massive stars, as being roughly 14,000 young stars. From this we suggest that the total population of young stars forming in the RCW 38 region is closer to $10^{4}$, making it one of the more massive known clusters within $2 \mathrm{kpc}$.

\subsection{Deeply Embedded Objects}

The MIPS $24 \mu \mathrm{m}$ mosaic was examined in a search for embedded protostars or young objects associated with emission regions. Three such emission regions were identified to the $\mathrm{N}$ of the cluster along the ridge of pre-main sequence stars forming the NW subcluster. The diffuse emission is associated with three stars not previously identified as YSOs, variable or O-star candidates. Their IRAC colors and SEDs suggest that they lack circumstellar material and that the emission arises from dust heating of the surrounding nebula. The coordinates and photometry of the three stars are given in Table 14.

A deeply embedded object was detected in the central subcluster, at coordinates $8^{h r} 59^{m} 05.95^{s}$, $-47^{d} 30^{m} 39.7^{s}$. It is offset from IRS 2 by $\sim 3.5^{\prime \prime}$ or $\sim 6000$ AU. This object was detected only in the $8.0 \mu \mathrm{m}$ band (no $24 \mu \mathrm{m}$ photometry is available due to saturation); it does not alter its position during the year between the Ep.2 and Ep.4 observations and hence it is assumed to be a deeply embedded protostar. It has a magnitude of $4.679 \pm 0.099$ at $8.0 \mu \mathrm{m}$ and is faintly visible at $5.8 \mu \mathrm{m}$, though not resolved as a point source. It is not detected at $4.5 \mu \mathrm{m}$ suggesting an absence of shocked molecular hydrogen emission, which would indicate that it is not a jet feature. Therefore we assume that this object is an embedded early protostar and may be embedded behind the O5.5 binary. A second object was also identified only at $8.0 \mu \mathrm{m}$ but was found to move from Ep.2 to Ep.4 and hence considered a contaminating foreground source.

\subsection{Subclustering and YSO Spatial Distribution}

The spatial distribution of young stellar objects in a cluster provides an insight into the fragmentation processes leading to the formation of protostellar cores, evidence for triggered star formation and the subsequent dynamical evolution of the stars as they evolve from the protostellar to the main sequence (?). While DeRose et al. (2009) examined the young stars in the inner core of the cluster at near-IR wavelengths, finding no evidence of subclustering, we will examine the extended molecular cloud. By including the longer mid-IR wavelengths we can obtain a clearer determination of the IR excess emission, and thereby more accurately 
classify the YSOs as protostellar, disk-bearing class II or diskless class III. These data provide a deeper understanding of the star formation history of the extended RCW 38 region.

\subsubsection{Spatial Distribution of Identified YSOs}

The spatial distribution of the young stars in the cluster in each evolutionary class was examined using a nearest neighbor technique. We define the nearest neighbor distance as the projected distance to the nearest YSO of the same class, assuming a distance to RCW 38 of $1.7 \mathrm{kpc}$. For each evolutionary class, $10 \mathrm{~K}$ random distributions of stars were generated, with the number of stars equal to the number of objects in the given evolutionary class. For the class III sources, the randomly distributed stars were constrained to fall within the Chandra field of view, for all other classes, the random distribution covered the region of the IR-field. The resulting nearest neighbor distributions of the observed YSOs and the random distributions were compared for each class using the K-S test. The probabilities that the random and observed distributions were drawn from the same parent distribution are listed in Table 15. The distributions of the class 0/I, flat spectrum and class II are found to be non-random. The class III sources show some evidence of mixing; this is likely a statistical effect of the smaller Chandra field of view.

Fig. 10 shows the nearest neighbor differential distributions by evolutionary class of the young stars. The open histograms show all sources in that class, while the filled histograms show the subsample of X-ray luminous stars. The upper plot shows the random distribution, averaged over the $10 \mathrm{~K}$ runs. The median spacing of the protostellar members was larger than that of the pre-main sequence objects. The median separation over the field for the class $0 / \mathrm{I}$ sources was $0.644 \mathrm{pc}$, and of the flat spectrum $0.433 \mathrm{pc}$. In comparison the pre-main sequence class II members had a median spacing of $0.249 \mathrm{pc}$, and the class III $0.346 \mathrm{pc}$.

Fig. 11 shows the spatial distribution of all identified YSOs as a function of their evolutionary class in the upper plots. The lower plots shows the spatial distribution of the young stars, again as a function of evolutionary class, in an expanded region centered on IRS 2. The detection of class III members is limited by the smaller field of view of the Chandra ACIS instrument. Evidence of subclustering and separate protostellar formation sites can be observed in the upper panels of Fig. 11- indicating that a unique centre of star formation is unlikely. An arc or filament of protostellar objects is also observed surrounding the central IRS 2 cavity. The formation of these young stars may have been influenced by their proximity to the IRS 2 binary. The spatial distributions of the class II and III stars surrounding IRS 2 do not trace this arc/filamentary structure. A SIMBA $1.2 \mathrm{~mm}$ map of the IRS 2 region is overplotted in the lower left of Fig. 11, and shows a ridge of emission which 
is spatially coincident with the filament of protostars (Vigil 2004). This implies that the protostars remain embedded in their natal, dense dust and gas while the pre-main sequence stars have dispersed through the cluster.

\subsubsection{Subclustering: Surface Density}

The stellar surface density of the YSOs was also calculated to determine the underlying structure of the cluster; the resulting map is shown in Fig. 12, The local surface density at each point on a uniform grid was calculated following the method outlined in Casertano \& Hut (1985):

$$
\sigma(i, j)=\frac{N-1}{\pi r_{N}^{2}(i, j)}
$$

where $r_{N}$ is the projected distance to the $N$ th nearest cluster member. In this case, the stellar surface density was calculated with a grid size of $100 \times 100$ using $N=18$, the distance to the 18th nearest neighbor, to smooth out smaller scale structure. Further, Casertano \& Hut (1985) found that the uncertainty goes as $\sigma /(N-2)^{1 / 2}$, so by taking $N=18$, the uncertainty is $25 \%$. The surface density plots show three subclusters surrounding the main IRS 2 core: a large subcluster to the SW, one to the NW, and a possible small grouping to the NE. The central cluster itself appears to have three density peaks which are likely real, though small, overdensities in the YSO population. One of these peaks corresponds to the region immediately surrounding IRS 2. The central subcluster coincides with the eastern cloud of Yamaguchi et al. (1999). The SW subcluster is coincident with the western cloud implying continued star formation in that region.

\subsubsection{Subclustering: Minimum Spanning Tree}

Another method of examining the spatial distribution and structure of a cluster is the minimum spanning tree (MST). The MST is defined as the set of branches or lines connecting a set of points such that the sum of the branch lengths is minimized and there are no closed loops. Fig. 13 shows the MST for the YSOs in RCW 38. The MST provides a better method of characterizing the separation lengths of sources as all points are connected in a single network, whereas the nearest neighbor technique tends to create small subgroupings due to pairing of adjacent points. The MST also has the advantage of not smoothing out geometry in the substructures. By assuming a critical MST branch length, the length where a break occurs in the cumulative distribution of branch lengths, substructures within the cluster 
can be defined. Following Gutermuth et al. (2009), in Fig. 14 we plot the distribution of branch lengths in the MST to obtain the characteristic branch length, found to be $0.412 \mathrm{pc}$. This was done by applying two linear fits to the data using the IDL routine linfit and minimizing the $\chi^{2}$ uncertainty of the two fits. In Fig. 13 the black branches are those with branch lengths less than the characteristic branch lengths while the gray branches are those with longer lengths. Those stars joined by black branches can be said to trace subclusters with the extended region. We note that the central core has two large subclusters to the NW and SW, and a third smaller subcluster to the NE, containing only eight stars, which may be a developing region.

Extending the usage of the MST, Cartwright \& Whitworth (2004) formulated the Qparameter, to quantify and distinguish between smooth large-scale radial density gradients and multiscale or fractal subclustering. This method provides a statistical method of characterizing structure in stellar clusters. The $Q$-parameter is defined as $\bar{m} / \bar{s}$, where $\bar{m}$ is the normalized mean MST branch length, $\bar{m}\left(N_{\text {total }} A\right)^{1 / 2} /\left(N_{\text {total }}-1\right)$, and $\bar{s}$ is the normalized mean separation, $\bar{s} / R_{\text {cluster }}$. A $Q$-parameter equal to 1 indicates a smooth radial distribution of sources, while values closer to zero indicate subclustering. For the RCW 38 region observed with IRAC, $\bar{m}=0.2219 \mathrm{pc}$ and $\bar{s}=0.5407 \mathrm{pc}$, yielding a value of $Q$ equal to 0.41 . This low value indicates that subclustering defines the structure and spatial distribution of the YSOs. DeRose et al. (2009) found a value of $Q$ in the smaller central IRS 2 region of 0.84, indicating that no subclustering was present on this scale. This result is consistent with our own since the DeRose et al. (2009) study covered an area of $\sim 0.5 \times 0.5 p c^{2}$, corresponding to the density peak containing IRS 2 within the central subcluster. On this smaller scale, any structure may be masked by the dynamical evolution of the young stars. On the larger scale the underlying subclustering of the complex is evident.

\subsubsection{The Subclusters}

The RCW 38 region has extended star formation over the $30^{\prime} \times 30^{\prime}$ IRAC field, a larger area than previously assumed. The central cluster where IRS $1 \& 2$ are located is surrounded by three subclusters and distributed star formation. Table 16 presents the populations of the subclusters, their median extinction and IRAC slope, and their protostellar and variable fractions.

Each of the subclusters contains variable stars; the NE subcluster contains just one, the NW contains eight, the SW three, and the central subcluster contains twenty-four. The remaining 36 form part of the distributed population. While the four subclusters contain very similar fractions of variables, the distributed population contains $\sim 50 \%$ more. The 
protostellar fractions of the NE and NW subclusters and the distributed population are similar at $\sim 18 \%$, while the the SW subcluster fraction is appreciably lower $(\sim 10 \%)$ and the central clusters is significantly higher at $\sim 26 \%$. The OB star candidates are distributed throughout the extended cluster region, c.f. Fig. 15, with 20/29 not located in one of the subclusters. Each of the four subclusters contains at least one massive star candidate (the central cluster contains at least 35: four new candidates in this study and 31 from Wolk et al. $(2006))$; indicating that star formation may have been initiated independently in each of the subcluster regions, with a subsequent generation (possibly due to triggering) in the central region around IRS 2.

The median $K$-band extinctions measured towards each subcluster and the distributed population are similar to within $1 \sigma$, consistent with all of the regions being at the same distance. Likewise, the median IRAC SED slope, dered $\alpha_{I R A C}$, are similar. This suggests a similar age and evolutionary history across the whole region. This further supports the idea that star formation is not propagating through the region, but rather that the molecular cloud has undergone collapse and is forming stars in fractal subclusters. Further spectroscopic observations may yet find a trend towards older ages in the distributed population similar to that observed in Serpens and NGC 1333 (Winston et al. 2009). The higher protostar fraction in the central cluster, rather than implying that the outer subclusters and distributed population are older per se, may be due to a second generation of protostars formed via triggering due to the IRS 2 O5.5 binary.

The north west subcluster of YSOs corresponds to the 2MASS cluster Obj 36 (Dutra \& Bica 2001), which is associated with the reflection nebula vdBH-RN43 (van de Bergh \& Herbst 1975) located at $08^{h} 58^{m} 04^{s},-47^{d} 22^{m} 50^{s}$ in the region of RCW 38. The association of this cluster with the main RCW 38 cluster has not previously been established. However, from the Spitzer observations, the subcluster does not appear to be physically isolated from the RCW 38 core; a band of YSOs stretches between the two. Visually, the structure of the molecular cloud appears contiguous between the two regions, c.f. Fig. 1. The ${ }^{13} \mathrm{CO}$ observations of Yamaguchi et al. (1999) also show emission extending between the regions. The K-band extinctions, $A_{K}$, and magnitudes at J-band and $3.6 \mu \mathrm{m}$ were compared to those of the core and other subclusters; no difference was found in either the range or median of the extinction or magnitudes between the NW subcluster and the rest of the region. This indicates that Obj 36 lies at a similar distance to the RCW 38 core and that it can be considered a subcluster in the extended RCW 38 star formation region. 


\section{Conclusion}

We have undertaken an extensive survey of the RCW 38 massive star forming region utilizing the capabilities of the Spitzer and Chandra space telescopes. Combining mid-IR and X-ray observations of this young star forming region, with near-IR data from 2MASS, has enabled the identification of YSOs from the protostellar and disk-bearing phases to diskless young members.

- We identify 624 young stellar objects in RCW 38 in the overlap regions of our four epochs of IRAC data. The initial identification process identified 712 objects, of which 32 were found to be contaminants. Of the remaining 680 YSOs, 56 lay outside the IRAC 4 band overlap region and were not included in the catalogue. Of the 624 YSOs, 226 were detected in X-rays with Chandra. The YSOs were of the following evolutionary classes: 23 class I, 90 flat spectrum, 437 class II, and 74 class III.

- A further 177 candidate YSOs were identified using the [3.6] vs. [3.6-5.8] colormagnitude diagram. Sources with $8.0 \leq m_{3.6} \leq 14.0$ and a color more than $1 \sigma$ greater than $[3.6-5.8]>0.4$ were selected. As the contamination removal methods could not be utilized (Gutermuth et al. 2009), we consider these to be tentative candidates.

- Of the 433 YSOs located in the Chandra field, 226 had confirmed X-ray detections. Nine of these were class $0 / \mathrm{I}$ protostars (9/22 or $41 \%$ of the class $0 / \mathrm{I}$ in the Chandra fov), eighteen were flat spectrum (18/58 or $31 \%$ of the flat spectrum sources), 125 were class II detections (125/279 or 45\%). The remaining 74 sources did not exhibit excess emission and were identified as class III members of the cluster; $74 / 624$ or $12 \%$ of the members were identified solely by Chandra.

- The disk fraction, the ratio of the number of X-ray detected disk-bearing pre-main sequence stars to the total number of X-ray detected pre-main sequence stars, of the YSOs with $\operatorname{mag}_{3.6}>12$ was found to be $63 \pm 6 \%$, consistent with a cluster age of $\sim 1$ Myrs.

- We find an observed relationship of $N_{H}=1.61 \pm 0.26 \times 10^{22} A_{K}$ from sixteen class II sources, and $N_{H}=1.52 \pm 0.49 \times 10^{22} A_{K}$ from eight class III objects. These values are consistent with the Vuong et al. (2003) ratio of $1.6 \times 10^{22}$ for the local ISM and nearby molecular clouds, and with the previous measurement of $2.0 \times 10^{22}$ (Wolk et al. 2006).

- By examining the photometric variance of the sources between epochs 72 variable candidates were identified. Of these 48 had previously been identified as YSOs from their IR excess emission and X-ray luminosity. The remaining 24 were found to consist 
of two class II sources and twenty-two candidate class IIIs. The percentage of identified variables by evolutionary class decreased through the class $0 /$ I sources $(30 \pm 11 \%)$ to the class II $(7 \pm 1 \%)$.

- An examination of the $K$ vs. $J-K$ color-magnitude diagram lead to the identification of 29 candidate O-star members of the cluster, of which nine were also identified as variables.

- Three young stars were identified by association with emission features at $24 \mu \mathrm{m}$; their IRAC colors and SEDs indicate that they are diskless class III members. Another deeply embedded protostar was identified in the centre of RCW 38, slightly offset from the O5.5 binary.

- The spatial distribution of the cluster was found to be fractally subclustered, with a $Q$-parameter of 0.41 . The MST of the cluster had a characteristic branch length of 0.412 pc. An extended filament of protostars in the central subcluster appears to remain embedded in dense dust and gas, which may trace the edge of density enhancement perhaps due to a shock front, and indicates ongoing and perhaps triggered star formation in the central core of RCW 38.

- We identify four subclusters in RCW 38; the central region of star formation in RCW 38 is surrounded by three subclusters and distributed young stars. Variable sources were identified in each of the four subclusters. The $\mathrm{O}$ star candidates are distributed throughout the cluster; candidates are located in each of the four subclusters, while twenty candidates form part of the distributed population. The central cluster and distributed stars have higher protostellar fractions than the three subclusters.

- The NW subcluster contains 62 members and is identified with a previously identified near-IR star cluster: Obj 36 (Dutra \& Bica 2001). Cloud morphology, continuous YSO distribution, and extinction measurements indicate that this region is not separate from, but is rather a subcluster of, RCW 38.

The authors would like to thank the referee for many helpful comments. This work is based on observations made with the Spitzer Space Telescope (PID 20127), which is operated by the Jet Propulsion Laboratory, California Institute of Technology under NASA contract 1407. Support for this work was provided by NASA through contract 1256790 issued by JPL/Caltech. Support for the IRAC instrument was provided by NASA through contract 960541 issued by JPL. This publication makes use of data products from the Two Micron All Sky Survey, which is a joint project of the University of Massachusetts and the Infrared Processing and Analysis Center/California Institute of Technology, funded by 
the National Aeronautics and Space Administration and the National Science Foundation. This research has made use of the NASA/IPAC Infrared Science Archive, which is operated by the Jet Propulsion Laboratory, California Institute of Technology, under contract with the National Aeronautics and Space Administration. This research made use of Montage, funded by the National Aeronautics and Space Administration's Earth Science Technology Office, Computation Technologies Project, under Cooperative Agreement Number NCC5626 between NASA and the California Institute of Technology. Montage is maintained by the NASA/IPAC Infrared Science Archive. TLB acknowledges support from NASA through a grant for HST program 11123 from the Space Telescope Science Institute, which is operated by the Association of Universities for Research in Astronomy, Incorporated, under NASA contract NAS5-26555. 


\section{REFERENCES}

Allen, L., Calvet, N., D’Alessio, P., et al, 2004, ApJS, 154, 363-366.

Andre, P., Montmerle, T., 1994, ApJ, 420, 837.

Baraffe, I., 1998, A\&A, 337, 403.

Blum, R.D., Mould, J.R., Olsen, K.A., et al., 2006, AJ, 132, 2034-2045.

Calvet, N., Hartmann, L., Kenyon, S.J., Whitney, B.A., 1994, ApJ, 434, 330.

Cardelli, J., Clayton, G., Mathis, j., 1989, ApJ, 345, 245.

Carpenter, J.M., Hillenbrand, L.A., Skrutskie, M.F., 2001, AJ, 121, 3160.

Cartwright, A., Whitworth, A., 2004, MNRAS, 348, 589.

Casanova, S., Montmerle, T., Feigelson, E.D., Andre, P., 1995, ApJ, 439, 752.

Casertano, S., Hut, P., 1985, ApJ, 298, 80.

Comerón, F., Schneider, N., Russeil, D., 2005, A\&A, 433, 955-977.

Damiani, F., Maggio, A., Micela, G., \& Sciortino, S. 1996, Astronomical Data Analysis Software and Systems V, 101, 143

D’Antona, F., Mazzitelli, I., 1997, Mem. S.A.It., 68, 807.

DeRose, K. L., Bourke, T. L., Gutermuth, R. A., et al., 2009, AJ, 138, 33.

Doppman, G.W., Greene T.P., Covey K.R., Lada C.J., 2005, ApJ, 130, 1145-1170.

Dutra, C.M., Bica, E., 2001, A\&A, 376, 434-440.

Eisenhardt, P.R.,Stern, D., Brodwin, M., et al., 2004, ApJS, 154, 48.

Evans, I. N., Primini, F.A., Glotfelty, K.J., Anderson, C.S., et al., 2010, ApJS, 189, 37

Favata, Giardino, G., Micela, G., et al., 2003, A\&A, 403, 187.

Favata, F., Flaccomio, E., Reale, F., et al. , 2005, ApJS, 160, 469.

Fazio, G.G., Hora, J.L, Allen L.E., et al., 2004, ApJS, 154, 10.

Feigelson, E., Townsley, L., 2008, ApJ, 673, 354. 
Feigelson, E., Getman, R., Townsley, L., et al., 2005, ApJS, 160, 379.

Feigelson, E. \& Getman, R., 2005, astro-ph/0501207.

Feigelson, E. D., Montmerle, T., 1999, ARA\&A, 37, 363-408.

Feigelson, E. D.; Kriss, G. A., 1981, ApJ, 248, L35-L38.

Flaherty, K.M., Pipher, J, L, Megeath, S.T., et al., 2007, ApJ, 663, 1069F.

Freeman, P. E., Kashyap, V., Rosner, R., \& Lamb, D. Q. 2002, ApJS, 138, 185

Frogel, J.A., Persson, S.E., 1974, ApJ, 192, 351.

Getman, K. V., Flaccomio E., Broos, P.S., et al., 2005, ApJS, 160, 319-352.

Getman, K. V., Feigelson, E. D., Townsley, L., et al., 2006, ApJS, 163, 306.

Giampapa, M.S., Rosner, R., Kashyap, V., et al., 1996, ApJ, 463, 707.

Gordon, K. D., Rieke, G.H., Engelbracht, C.W., et al. 2005, PASP, 117, 503.

Greene, T.P., Wilking, B.A., Andre, P., et al., 1994, ApJ, 434, 614.

Gum, C.S., 1955, MNRAS, 67, 155.

Gutermuth, R., Megeath, S.T., Myers, P.C., et al., 2009, ApJS, 184, 18G.

Gutermuth, R., Myers, P.C., Megeath, S.T., et al., 2008, ApJ, 674, 336.

Gutermuth, R., 2005, PhD Thesis, University of Rochester.

Gutermuth, R., Megeath, S. T., Pipher, J., et al., 2005, ApJ, 632, 397-420.

Gutermuth, R., Megeath, S. T., Muzerolle, J., et al., 2004, ApJS, 154, 374.

Haisch, K. E. Jr., Lada, E. A. \& Lada, C. J. 2001, ApJ, 553, L153.

Harvey, P., Chapman N., Lai S-P., 2005, ApJ, 644, 30-325.

Hayashi, M.R., Hibata, K., Matsumoto. R., 1996, ApJ, 468, L37.

Hernadez, J., Briceno, C., Calvet, N., et al., 2006, ApJ, 652, 472.

Hernadez, J., Clavet, N., Briceno, C., et al., 2007, ApJ, 662, 1067.

Isobe, H., Shibata, K., Yokoyama, T., Imanishi, K., 2003, PASJ, 55, 967. 
Jorgensen, J., Harvey, P., Evans, N.J., et al, 2006, ApJ, 645, 1246-1263.

Kastner, J.H., Huenemoerder, D.P., Schulz, N.S., et al., 2002, ApJ, 567, 434.

Kenyon, S. J., Calvet, N., Hartmann, L., 1993, ApJ, 414, 676.

Knodlseder, J., 2000, A\&A, 360, 539.

Küker, M., \& Rüdiger, G., 1999, A\&A, 346, 922.

Lada, C.J., Wilking. B.A., 1984, ApJ, 287, 610-621.

Lada, C.J., IAU Symp. 115, 1987, Star Forming Regions ed. M Peimbert \& J. Jugaku.

Lada, C.J., Luhman, K., Muench, A., et al., 2006, AJ, 131, 1574.

Landsman, W.B., 1989, BAAS, Vol. 21, p784.

Megeath, S.T., Gutermuth R.A., Allen L.E., et al., 2004, ApJS, 154, 367.

Morrison, R., McCammon, D., 1983, ApJ, 270, 119-122.

Muench, A., Getman, K., Hillenbrand, L., Preibisch, T., 2008, in "Handbook of Star Forming Regions Vol.I", ASP, editor: Reipurth, B..

Preibisch, T., Feigelson, E., 2005, ApJS, 160, 390.

Reach, W.T., Megeath, S.T., Cohen, M., et al., 2005, PASP, 117, 978-990.

Rieke, G.H., Young, E.T., Engelbracht, C.W., et al., 2004, ApJS, 154, 25-29.

Rodgers, A.W., Campbell, C.T., Whiteoak, J.B., 1960, MNRAS, 121, 103.

Romanova, M.M., Ustyugova, G.V., Koldoba, A.V., Lovelace, R.V.E, 2004, ApJ, 616, L151.

Ryter, Ch. E., 1996, 1996, Ap\&SS, 236, 285-291.

Siess, L., Dufour, E., Forestini, M., 2000, A\&A, 358, 593-599.

Skrutskie, M.F., Cutri R.M., Stiening R., et al., 2006, AJ, 131, 1163.

Smith, C.H., Bourke, T.L., Wright, C.M., et al., 1999, MNRAS, 303, 367-379.

Smith, R.K., Brickhouse, N.S., Liedahl, D.A., Raymond, J.C., 2001, ApJ, 556, L91-L95.

Stepnik, B., Abergel, A., Bernard, J-P, et al. 2003, A\&A, 398, 551. 
Stern, R.A., Schmitt, J.H.M.M., Pye, J.P., et al., 1994, ApJ, 427, 808.

Telleschi, A., Gudel, M., Briggs, K.R., Audard, M., Palla, F., 2007, A\&A, 468, 425.

van deBergh, S., Herbst, W., 1975, AJ, 80, 208.

Vigil M., 2004, M.Sc. Thesis, Massachusetts Institute of Technology.

Vuong, M. H., Montmerle, T., Grosso, N., et al., 2003, A\&A, 408, 581-599.

Wang, J., Townsley, L.K., Feigelson, E.D., et al., 2008, ApJ, 675, 464-490.

Wang, J., Feigelson, E.D., Townsley, L.K., et al., 2011, arXiv1103.0785W.

Whitney, B. A., Wood, K., Bjorkman, J. E., Wolff, M. J., 2003, ApJ, 591, 1049-1063.

Whitney, B. A., Wood, K., Bjorkman, J. E., Cohen, M., 2003, ApJ, 598, 1079-1099.

Winston, E., Megeath, S.T., Wolk, S.J., et al., 2007, ApJ, 669, 493.

Winston, E., Megeath, S.T., Wolk, S.J., et al. 2009, AJ, 137, 4777.

Winston, E., Megeath, S.T., Wolk, S.J., et al., 2010, AJ, 140, 266.

Wolk, S. J., Spitzbart, B. D., Bourke, T. L., Alves, J., 2006, AJ, 132, 1100-1125.

Wolk, S. J., Bourke, T. L., Vigil, M., 2008, in "Handbook of Star Forming Regions Vol.II", ASP, editor: Reipurth, B..

Wolk, S. J., Spitzbart, B., Bourke, T.L., et al., 2008, AJ, 135, 693.

Wolk, S. J., Winston, E., Bourke, T. L., et al., 2010, ApJ, 715, 671.

Wolk, S.J., Broos, P.S., Getman, K.V., et al., 2011, arXiv1103.1126W.

Yamaguchi, R., Saito, H., Mizuno, N., et al., 1999, PASJ, 51, 791. 
Table 1. Summary of IRAC Epochs.

\begin{tabular}{rcrrrr}
\hline \hline \multirow{2}{*}{ Epoch ID } & Date & Int. Time (s) & RA (J2000) & Dec (J2000) & Coverage (arcmin) \\
\hline 1 & $2006.06 .0505: 53: 33$ & 12 & $8: 58: 59.7$ & $-47: 26: 37.6$ & $\sim 30 \times 30$ \\
2 & $2006.06 .0506: 58: 53$ & 2 & $8: 59: 25.9$ & $-47: 32: 34.8$ & $\sim 15 \times 15$ \\
3 & $2007.02 .2119: 42: 03$ & 12 & $8: 59: 05.7$ & $-47: 30: 43.6$ & $\sim 30 \times 30$ \\
4 & $2007.02 .2120: 45: 35$ & 2 & $8: 59: 05.9$ & $-47: 30: 38.7$ & $\sim 15 \times 15$ \\
\hline
\end{tabular}


Table 2. NGC 1333: Chandra X-ray Source Photometry

\begin{tabular}{|c|c|c|c|c|c|c|c|c|c|}
\hline $\begin{array}{l}\text { Chandra } \\
\text { ID }\end{array}$ & $\begin{array}{c}\text { RA } \\
(2000)\end{array}$ & $\begin{array}{c}\text { Dec } \\
(2000)\end{array}$ & $\begin{array}{l}\text { Raw } \\
\text { CNT }\end{array}$ & $\begin{array}{l}\text { Net }^{a} \\
\text { CNT }\end{array}$ & $\begin{array}{c}N_{H} \\
10^{22} \mathrm{~cm}^{-2}\end{array}$ & $\begin{array}{l}\mathrm{kT} \\
\mathrm{keV}\end{array}$ & $\begin{array}{l}\text { Abs. Flux } \\
{[e r}\end{array}$ & $\begin{array}{l}\text { Tot. UnAbs. Flux } \\
\left.\mathrm{gcm}^{-2} s^{-1}\right]\end{array}$ & $\chi^{2}$ \\
\hline 1 & $8: 59: 55.58$ & $-47: 28: 28.80$ & 12.0 & -0.3 & $\ldots$ & & $\cdots$ & $\ldots$ & $\ldots$ \\
\hline 2 & $8: 59: 53.60$ & $-47: 28: 46.31$ & 93.0 & 89.5 & $1.87 \pm 0.36$ & $3.90 \pm 1.54$ & $1.45 \mathrm{E}-14$ & $3.28 \mathrm{E}-14$ & 0.53 \\
\hline 3 & $8: 59: 52.33$ & $-47: 34: 01.49$ & 15.0 & 3.2 & $0.00 \pm 0.17$ & $1.83 \pm 36.36$ & $1.14 \mathrm{E}-15$ & $1.14 \mathrm{E}-15$ & 0.60 \\
\hline 4 & $8: 59: 50.36$ & $-47: 26: 57.03$ & 68.0 & 63.5 & $0.35 \pm 0.17$ & $3.12 \pm 2.14$ & $6.54 \mathrm{E}-15$ & $1.02 \mathrm{E}-14$ & 0.57 \\
\hline 5 & $8: 59: 47.99$ & $-47: 28: 56.23$ & 7.0 & 3.4 & $0.00 \pm 0.27$ & $2.32 \pm \ldots$ & $4.18 \mathrm{E}-16$ & $4.18 \mathrm{E}-16$ & 0.37 \\
\hline 6 & $8: 59: 46.70$ & $-47: 31: 53.62$ & 71.0 & 67.0 & $0.75 \pm 0.25$ & $4.63 \pm 5.88$ & $8.52 \mathrm{E}-15$ & $1.42 \mathrm{E}-14$ & 0.57 \\
\hline 7 & $8: 59: 42.96$ & $-47: 31: 58.63$ & 38.0 & 34.1 & $1.90 \pm 0.81$ & $7.54 \pm \ldots$ & $6.32 \mathrm{E}-15$ & $1.18 \mathrm{E}-14$ & 0.54 \\
\hline 8 & $8: 59: 41.58$ & $-47: 35: 07.91$ & 177.0 & 164.2 & $0.81 \pm 0.15$ & $3.02 \pm 0.85$ & $1.86 \mathrm{E}-14$ & $3.63 \mathrm{E}-14$ & 0.87 \\
\hline 9 & $8: 59: 41.47$ & $-47: 26: 08.09$ & 13.0 & 9.7 & $100.00 \pm 88.20$ & $10.00 \pm \ldots$ & $2.16 \mathrm{E}-15$ & $5.98 \mathrm{E}-14$ & 0.43 \\
\hline 10 & $8: 59: 39.22$ & $-47: 30: 37.47$ & 23.0 & 21.3 & $3.24 \pm 1.63$ & $10.00 \pm \ldots$ & $4.80 \mathrm{E}-15$ & $9.87 \mathrm{E}-15$ & 0.34 \\
\hline 11 & $8: 59: 38.66$ & $-47: 29: 18.37$ & 7.0 & 5.8 & $0.00 \pm 0.10$ & $0.15 \pm 0.04$ & $1.50 \mathrm{E}-15$ & $1.50 \mathrm{E}-15$ & 0.20 \\
\hline 12 & $8: 59: 38.32$ & $-47: 32: 01.58$ & 7.0 & 6.0 & $2.54 \pm 2.40$ & $3.71 \pm \ldots$ & $1.40 \mathrm{E}-15$ & $3.57 \mathrm{E}-15$ & 0.22 \\
\hline 13 & $8: 59: 37.44$ & $-47: 31: 25.60$ & 14.0 & 12.6 & $0.36 \pm 0.69$ & $7.54 \pm \ldots$ & $1.55 \mathrm{E}-15$ & $2.09 \mathrm{E}-15$ & 0.28 \\
\hline 14 & $8: 59: 37.37$ & $-47: 34: 35.39$ & 22.0 & 18.4 & $0.96 \pm 0.76$ & $5.96 \pm \ldots$ & $2.92 \mathrm{E}-15$ & $4.86 \mathrm{E}-15$ & 0.45 \\
\hline 15 & $8: 59: 36.92$ & $-47: 33: 04.80$ & 63.0 & 61.9 & $1.75 \pm 0.44$ & $3.95 \pm 2.08$ & $9.71 \mathrm{E}-15$ & $2.14 \mathrm{E}-14$ & 0.37 \\
\hline 16 & $8: 59: 36.78$ & $-47: 29: 21.78$ & 11.0 & 9.8 & $2.55 \pm 1.18$ & $0.94 \pm 0.38$ & $8.51 \mathrm{E}-16$ & $1.08 \mathrm{E}-14$ & 0.22 \\
\hline 17 & $8: 59: 35.84$ & $-47: 29: 11.97$ & 10.0 & 8.8 & $10.20 \pm 2.41$ & $0.45 \pm 0.05$ & $1.03 \mathrm{E}-15$ & $1.19 \mathrm{E}-12$ & 0.23 \\
\hline 18 & $8: 59: 35.58$ & $-47: 31: 37.57$ & 18.0 & 17.1 & $1.39 \pm 0.61$ & $2.00 \pm 1.68$ & $2.43 \mathrm{E}-15$ & $7.16 \mathrm{E}-15$ & 0.23 \\
\hline 19 & $8: 59: 35.39$ & $-47: 28: 18.32$ & 69.0 & 68.3 & $1.69 \pm 0.33$ & $2.09 \pm 0.57$ & $1.29 \mathrm{E}-14$ & $4.00 \mathrm{E}-14$ & 0.31 \\
\hline 20 & $8: 59: 35.36$ & $-47: 27: 23.51$ & 102.0 & 100.4 & $0.80 \pm 0.15$ & $1.63 \pm 0.39$ & $8.57 \mathrm{E}-15$ & $2.26 \mathrm{E}-14$ & 0.39 \\
\hline 21 & $8: 59: 35.22$ & $-47: 26: 32.10$ & 60.0 & 55.9 & $4.25 \pm 1.19$ & $4.70 \pm 2.58$ & $1.18 \mathrm{E}-14$ & $3.27 \mathrm{E}-14$ & 0.61 \\
\hline 22 & $8: 59: 34.83$ & $-47: 28: 25.32$ & 6.0 & 5.2 & $4.30 \pm 1.30$ & $0.33 \pm 0.06$ & $5.61 \mathrm{E}-16$ & $3.40 \mathrm{E}-13$ & 0.15 \\
\hline 23 & $8: 59: 34.77$ & $-47: 28: 40.29$ & 18.0 & 17.0 & $4.23 \pm 1.79$ & $3.24 \pm 2.41$ & $3.92 \mathrm{E}-15$ & $1.33 \mathrm{E}-14$ & 0.24 \\
\hline 24 & $8: 59: 34.43$ & $-47: 30: 53.63$ & 9.0 & 7.6 & $0.00 \pm 0.05$ & $1.22 \pm 1.94$ & $5.82 \mathrm{E}-16$ & $5.83 \mathrm{E}-16$ & 0.25 \\
\hline 25 & $8: 59: 33.32$ & $-47: 32: 54.05$ & 30.0 & 29.0 & $0.97 \pm 0.34$ & $1.82 \pm 0.99$ & $2.66 \mathrm{E}-15$ & $7.13 \mathrm{E}-15$ & 0.29 \\
\hline 26 & $8: 59: 32.46$ & $-47: 32: 36.11$ & 21.0 & 19.6 & $0.37 \pm 0.28$ & $2.15 \pm 1.88$ & $1.66 \mathrm{E}-15$ & $2.92 \mathrm{E}-15$ & 0.29 \\
\hline 27 & $8: 59: 31.63$ & $-47: 31: 56.97$ & 38.0 & 36.4 & $1.16 \pm 0.97$ & $10.00 \pm \ldots$ & $5.64 \mathrm{E}-15$ & $9.03 \mathrm{E}-15$ & 0.40 \\
\hline 28 & $8: 59: 31.61$ & $-47: 26: 08.42$ & 8.0 & 4.4 & $0.00 \pm 0.18$ & $0.47 \pm 0.64$ & $5.78 \mathrm{E}-16$ & $5.78 \mathrm{E}-16$ & 0.38 \\
\hline 29 & $8: 59: 31.55$ & $-47: 33: 50.17$ & 20.0 & 16.9 & $0.73 \pm 0.53$ & $2.32 \pm 3.29$ & $1.56 \mathrm{E}-15$ & $3.28 \mathrm{E}-15$ & 0.39 \\
\hline 30 & $8: 59: 31.27$ & $-47: 33: 30.74$ & 99.0 & 97.6 & $0.00 \pm 0.02$ & $1.06 \pm 0.10$ & $6.72 \mathrm{E}-15$ & $6.72 \mathrm{E}-15$ & 0.37 \\
\hline 31 & $8: 59: 30.60$ & $-47: 28: 00.04$ & 12.0 & 10.7 & $1.70 \pm 0.96$ & $2.58 \pm 3.73$ & $1.40 \mathrm{E}-15$ & $3.78 \mathrm{E}-15$ & 0.25 \\
\hline 32 & $8: 59: 30.00$ & $-47: 26: 34.10$ & 27.0 & 26.0 & $0.62 \pm 0.36$ & $4.43 \pm 44.13$ & $3.13 \mathrm{E}-15$ & $5.07 \mathrm{E}-15$ & 0.27 \\
\hline 33 & $8: 59: 29.94$ & $-47: 31: 50.20$ & 57.0 & 55.5 & $4.24 \pm 0.63$ & $1.35 \pm 0.19$ & $7.43 \mathrm{E}-15$ & $6.71 \mathrm{E}-14$ & 0.37 \\
\hline 34 & $8: 59: 29.03$ & $-47: 27: 57.04$ & 8.0 & 6.4 & $0.00 \pm 0.07$ & $2.32 \pm \ldots$ & $5.37 \mathrm{E}-16$ & $5.38 \mathrm{E}-16$ & 0.24 \\
\hline 35 & $8: 59: 28.92$ & $-47: 32: 17.89$ & 13.0 & 12.1 & $0.69 \pm 0.16$ & $0.19 \pm 0.02$ & $7.05 \mathrm{E}-16$ & $3.62 \mathrm{E}-14$ & 0.17 \\
\hline 36 & $8: 59: 28.92$ & $-47: 31: 40.59$ & 121.0 & 119.0 & $1.40 \pm 0.28$ & $3.84 \pm 1.41$ & $1.59 \mathrm{E}-14$ & $3.30 \mathrm{E}-14$ & 0.53 \\
\hline 37 & $8: 59: 28.79$ & $-47: 32: 22.35$ & 72.0 & 70.7 & $1.28 \pm 0.43$ & $7.54 \pm 30.69$ & $1.08 \mathrm{E}-14$ & $1.83 \mathrm{E}-14$ & 0.40 \\
\hline 38 & $8: 59: 28.63$ & $-47: 29: 37.97$ & 16.0 & 15.1 & $1.29 \pm 0.56$ & $1.64 \pm 1.15$ & $3.05 \mathrm{E}-15$ & $1.01 \mathrm{E}-14$ & 0.21 \\
\hline 39 & $8: 59: 28.33$ & $-47: 32: 14.16$ & 7.0 & 5.9 & $0.00 \pm 1.27$ & $10.00 \pm \ldots$ & $5.86 \mathrm{E}-16$ & $5.86 \mathrm{E}-16$ & 0.19 \\
\hline 40 & $8: 59: 28.32$ & $-47: 26: 20.00$ & 111.0 & 107.1 & $1.30 \pm 0.30$ & $10.00 \pm 28.84$ & $1.88 \mathrm{E}-14$ & $3.08 \mathrm{E}-14$ & 0.63 \\
\hline 41 & $8: 59: 28.22$ & $-47: 35: 45.75$ & 14.0 & 10.3 & $2.40 \pm 1.48$ & $2.00 \pm 2.18$ & $1.26 \mathrm{E}-15$ & $4.81 \mathrm{E}-15$ & 0.39 \\
\hline 42 & $8: 59: 28.18$ & $-47: 29: 26.14$ & 51.0 & 50.1 & $1.37 \pm 0.32$ & $1.97 \pm 0.69$ & $1.30 \mathrm{E}-14$ & $3.86 \mathrm{E}-14$ & 0.31 \\
\hline 43 & $8: 59: 27.84$ & $-47: 28: 03.19$ & 19.0 & 17.2 & $0.65 \pm 0.41$ & $2.53 \pm 4.73$ & $1.65 \mathrm{E}-15$ & $3.24 \mathrm{E}-15$ & 0.30 \\
\hline 44 & $8: 59: 27.02$ & $-47: 34: 10.56$ & 9.0 & 4.4 & $0.03 \pm 0.10$ & $0.11 \pm 0.02$ & $2.38 \mathrm{E}-15$ & $3.64 \mathrm{E}-15$ & 0.42 \\
\hline 45 & $8: 59: 26.68$ & $-47: 33: 10.15$ & 11.0 & 9.9 & $1.91 \pm 0.81$ & $1.14 \pm 0.59$ & $8.69 \mathrm{E}-16$ & $5.57 \mathrm{E}-15$ & 0.23 \\
\hline
\end{tabular}


Table 2-Continued

\begin{tabular}{|c|c|c|c|c|c|c|c|c|c|}
\hline $\begin{array}{l}\text { Chandra } \\
\text { ID }\end{array}$ & $\begin{array}{c}\text { RA } \\
(2000)\end{array}$ & $\begin{array}{c}\text { Dec } \\
(2000)\end{array}$ & $\begin{array}{l}\text { Raw } \\
\text { CNT }\end{array}$ & $\begin{array}{l}\text { Net }^{a} \\
\text { CNT }\end{array}$ & $\begin{array}{c}N_{H} \\
10^{22} \mathrm{~cm}^{-2}\end{array}$ & $\begin{array}{l}\mathrm{kT} \\
\mathrm{keV}\end{array}$ & \multicolumn{2}{|c|}{$\left[\mathrm{ergcm}^{-2} \mathrm{~s}^{-1}\right]$} & $\chi^{2}$ \\
\hline 46 & $8: 59: 26.55$ & $-47: 30: 07.95$ & 32.0 & 31.4 & $2.06 \pm 0.45$ & $1.23 \pm 0.30$ & $5.35 \mathrm{E}-15$ & $3.25 \mathrm{E}-14$ & 0.24 \\
\hline 47 & $8: 59: 26.25$ & $-47: 31: 03.95$ & 9.0 & 7.7 & $1.38 \pm 1.53$ & $10.00 \pm$ & $1.20 \mathrm{E}-15$ & $1.99 \mathrm{E}-15$ & 0.25 \\
\hline 48 & $8: 59: 26.23$ & $-47: 27: 54.64$ & 28.0 & 26.8 & $1.70 \pm 0.49$ & $1.65 \pm 0.71$ & $3.03 \mathrm{E}-15$ & $1.15 \mathrm{E}-14$ & 0.31 \\
\hline 49 & $8: 59: 25.93$ & $-47: 27: 26.15$ & 12.0 & 10.3 & $4.67 \pm 3.17$ & $1.82 \pm 0.98$ & $1.68 \mathrm{E}-15$ & $1.03 \mathrm{E}-14$ & 0.27 \\
\hline 50 & $8: 59: 25.80$ & $-47: 34: 36.69$ & 22.0 & 18.6 & $0.00 \pm 0.15$ & $1.19 \pm 2.97$ & $1.27 \mathrm{E}-15$ & $1.27 \mathrm{E}-15$ & 0.39 \\
\hline 51 & $8: 59: 25.06$ & $-47: 27: 26.91$ & 13.0 & 11.4 & $3.03 \pm 1.49$ & $2.67 \pm 2.23$ & $1.96 \mathrm{E}-15$ & $6.59 \mathrm{E}-15$ & 0.25 \\
\hline 52 & $8: 59: 24.38$ & $-47: 34: 36.28$ & 13.0 & 9.2 & $0.00 \pm 0.11$ & $2.93 \pm 39.86$ & $9.38 \mathrm{E}-16$ & $9.38 \mathrm{E}-16$ & 0.38 \\
\hline 53 & $8: 59: 24.37$ & $-47: 31: 11.46$ & 17.0 & 15.9 & $1.95 \pm 1.12$ & $8.03 \pm \ldots$ & $2.83 \mathrm{E}-15$ & $5.26 \mathrm{E}-15$ & 0.27 \\
\hline 54 & $8: 59: 24.37$ & $-47: 30: 44.35$ & 18.0 & 17.1 & $6.79 \pm 2.69$ & $2.08 \pm 0.74$ & $3.35 \mathrm{E}-15$ & $2.20 \mathrm{E}-14$ & 0.24 \\
\hline 55 & $8: 59: 23.92$ & $-47: 28: 11.76$ & 6.0 & 4.5 & $7.50 \pm 4.22$ & $1.14 \pm 0.40$ & $8.40 \mathrm{E}-16$ & $1.84 \mathrm{E}-14$ & 0.24 \\
\hline 56 & $8: 59: 23.61$ & $-47: 26: 50.11$ & 19.0 & 17.4 & $0.51 \pm 0.38$ & $2.93 \pm 5.06$ & $1.89 \mathrm{E}-15$ & $3.28 \mathrm{E}-15$ & 0.31 \\
\hline 57 & $8: 59: 23.67$ & $-47: 25: 23.68$ & 23.0 & 18.7 & $0.42 \pm 0.32$ & $2.03 \pm 3.20$ & $1.57 \mathrm{E}-15$ & $2.94 \mathrm{E}-15$ & 0.49 \\
\hline 58 & $8: 59: 23.56$ & $-47: 35: 51.80$ & 66.0 & 62.6 & $2.03 \pm 0.43$ & $2.93 \pm 1.03$ & $9.71 \mathrm{E}-15$ & $2.59 \mathrm{E}-14$ & 0.53 \\
\hline 59 & $8: 59: 22.86$ & $-47: 26: 33.91$ & 41.0 & 39.5 & $1.10 \pm 0.42$ & $5.47 \pm 30.75$ & $5.71 \mathrm{E}-15$ & $1.00 \mathrm{E}-14$ & 0.37 \\
\hline 60 & $8: 59: 22.54$ & $-47: 28: 49.73$ & 10.0 & 8.8 & $3.33 \pm 2.85$ & $10.00 \pm \ldots$ & $1.92 \mathrm{E}-15$ & $3.98 \mathrm{E}-15$ & 0.25 \\
\hline 61 & $8: 59: 22.22$ & $-47: 25: 50.73$ & 27.0 & 22.4 & $1.30 \pm 0.39$ & $1.14 \pm 0.42$ & $1.79 \mathrm{E}-15$ & $8.63 \mathrm{E}-15$ & 0.43 \\
\hline 62 & $8: 59: 21.90$ & $-47: 30: 38.49$ & 36.0 & 35.3 & $2.08 \pm 0.49$ & $1.65 \pm 0.51$ & $6.84 \mathrm{E}-15$ & $2.90 \mathrm{E}-14$ & 0.27 \\
\hline 63 & $8: 59: 21.82$ & $-47: 36: 11.11$ & 10.0 & 6.9 & $0.00 \pm 0.05$ & $1.44 \pm 3.33$ & $6.34 \mathrm{E}-16$ & $6.34 \mathrm{E}-16$ & 0.33 \\
\hline 64 & $8: 59: 21.71$ & $-47: 32: 34.25$ & 20.0 & 18.5 & $4.97 \pm 1.66$ & $1.83 \pm 0.63$ & $2.95 \mathrm{E}-15$ & $1.88 \mathrm{E}-14$ & 0.30 \\
\hline 65 & $8: 59: 21.57$ & $-47: 29: 29.11$ & 331.0 & 329.3 & $2.43 \pm 0.20$ & $2.61 \pm 0.28$ & $4.66 \mathrm{E}-14$ & $1.44 \mathrm{E}-13$ & 0.60 \\
\hline 66 & $8: 59: 21.33$ & $-47: 33: 25.12$ & 19.0 & 17.7 & $4.91 \pm 2.68$ & $10.00 \pm \ldots$ & $4.23 \mathrm{E}-15$ & $9.89 \mathrm{E}-15$ & 0.30 \\
\hline 67 & $8: 59: 21.30$ & $-47: 25: 55.91$ & 120.0 & 114.9 & $1.11 \pm 0.22$ & $3.54 \pm 1.25$ & $1.50 \mathrm{E}-14$ & $3.02 \mathrm{E}-14$ & 0.56 \\
\hline 68 & $8: 59: 21.17$ & $-47: 31: 12.45$ & 8.0 & 7.1 & $4.36 \pm 2.10$ & $1.14 \pm 0.44$ & $1.55 \mathrm{E}-15$ & $1.99 \mathrm{E}-14$ & 0.15 \\
\hline 69 & $8: 59: 20.93$ & $-47: 29: 14.29$ & 50.0 & 48.7 & $1.11 \pm 0.30$ & $1.84 \pm 0.76$ & $4.79 \mathrm{E}-15$ & $1.35 \mathrm{E}-14$ & 0.37 \\
\hline 70 & $8: 59: 20.89$ & $-47: 31: 19.28$ & 7.0 & 5.6 & $3.00 \pm 1.90$ & $1.44 \pm 1.29$ & $1.02 \mathrm{E}-15$ & $6.39 \mathrm{E}-15$ & 0.19 \\
\hline 71 & $8: 59: 20.69$ & $-47: 26: 17.49$ & 68.0 & 66.4 & $1.20 \pm 0.20$ & $1.20 \pm 0.21$ & $5.29 \mathrm{E}-15$ & $2.28 \mathrm{E}-14$ & 0.34 \\
\hline 72 & $8: 59: 20.27$ & $-47: 31: 06.17$ & 8.0 & 7.1 & $2.57 \pm 2.64$ & $10.00 \pm \ldots$ & $3.38 \mathrm{E}-15$ & $6.50 \mathrm{E}-15$ & 0.18 \\
\hline 73 & $8: 59: 20.22$ & $-47: 28: 29.38$ & 86.0 & 84.8 & $3.44 \pm 0.60$ & $3.17 \pm 0.80$ & $1.50 \mathrm{E}-14$ & $4.72 \mathrm{E}-14$ & 0.44 \\
\hline 74 & $8: 59: 20.01$ & $-47: 34: 13.87$ & 4.0 & 2.6 & $0.00 \pm 0.10$ & $0.07 \pm 0.01$ & $3.85 \mathrm{E}-15$ & $3.86 \mathrm{E}-15$ & 0.21 \\
\hline 75 & $8: 59: 19.95$ & $-47: 29: 35.20$ & 18.0 & 17.0 & $0.64 \pm 0.15$ & $0.35 \pm 0.06$ & $1.89 \mathrm{E}-15$ & $2.22 \mathrm{E}-14$ & 0.19 \\
\hline 76 & $8: 59: 19.84$ & $-47: 27: 31.05$ & 7.0 & 4.9 & $2.89 \pm 17.43$ & $9.16 \pm \cdots$ & $9.50 \mathrm{E}-16$ & $1.91 \mathrm{E}-15$ & 0.31 \\
\hline 77 & $8: 59: 19.59$ & $-47: 29: 03.57$ & 10.0 & 8.5 & $0.00 \pm 0.18$ & $1.60 \pm 53.10$ & $6.54 \mathrm{E}-16$ & $6.55 \mathrm{E}-16$ & 0.26 \\
\hline 78 & $8: 59: 19.58$ & $-47: 33: 28.61$ & 12.0 & 10.6 & $0.90 \pm 1.29$ & $10.00 \pm \ldots$ & $1.59 \mathrm{E}-15$ & $2.42 \mathrm{E}-15$ & 0.27 \\
\hline 79 & $8: 59: 19.56$ & $-47: 27: 54.80$ & 11.0 & 9.6 & $6.38 \pm 1.86$ & $0.80 \pm 0.17$ & $1.32 \mathrm{E}-15$ & $7.69 \mathrm{E}-14$ & 0.26 \\
\hline 80 & $8: 59: 19.30$ & $-47: 29: 28.73$ & 22.0 & 21.0 & $3.28 \pm 1.13$ & $2.35 \pm 1.13$ & $5.80 \mathrm{E}-15$ & $2.23 \mathrm{E}-14$ & 0.25 \\
\hline 81 & $8: 59: 19.14$ & $-47: 32: 55.22$ & 7.0 & 5.6 & $0.00 \pm 0.07$ & $1.44 \pm 3.17$ & $4.95 \mathrm{E}-16$ & $4.95 \mathrm{E}-16$ & 0.24 \\
\hline 82 & $8: 59: 18.95$ & $-47: 32: 25.31$ & 40.0 & 38.7 & $1.12 \pm 0.39$ & $4.30 \pm 6.35$ & $5.52 \mathrm{E}-15$ & $1.04 \mathrm{E}-14$ & 0.35 \\
\hline 83 & $8: 59: 18.61$ & $-47: 25: 56.37$ & 31.0 & 25.9 & $0.00 \pm 0.06$ & $1.44 \pm 0.81$ & $2.25 \mathrm{E}-15$ & $2.26 \mathrm{E}-15$ & 0.47 \\
\hline 84 & $8: 59: 18.10$ & $-47: 31: 17.59$ & 14.0 & 12.4 & $14.01 \pm 8.77$ & $4.70 \pm 15.23$ & $4.08 \mathrm{E}-15$ & $1.99 \mathrm{E}-14$ & 0.29 \\
\hline 85 & $8: 59: 17.75$ & $-47: 28: 51.64$ & 69.0 & 67.5 & $0.77 \pm 0.26$ & $9.75 \pm 33.19$ & $1.01 \mathrm{E}-14$ & $1.50 \mathrm{E}-14$ & 0.43 \\
\hline 86 & $8: 59: 17.57$ & $-47: 27: 48.30$ & 17.0 & 15.0 & $1.87 \pm 0.62$ & $1.17 \pm 0.49$ & $1.36 \mathrm{E}-15$ & $8.32 \mathrm{E}-15$ & 0.26 \\
\hline 87 & $8: 59: 17.55$ & $-47: 27: 15.56$ & 9.0 & 7.2 & $0.00 \pm 0.06$ & $0.12 \pm 0.02$ & $3.59 \mathrm{E}-15$ & $3.60 \mathrm{E}-15$ & 0.26 \\
\hline 88 & $8: 59: 17.35$ & $-47: 26: 55.68$ & 8.0 & 6.4 & $0.33 \pm 0.14$ & $0.11 \pm 0.02$ & $7.99 \mathrm{E}-16$ & $2.33 \mathrm{E}-14$ & 0.23 \\
\hline 89 & $8: 59: 17.26$ & $-47: 30: 11.41$ & 49.0 & 47.0 & $5.32 \pm 1.13$ & $2.49 \pm 0.62$ & $8.65 \mathrm{E}-15$ & $4.06 \mathrm{E}-14$ & 0.39 \\
\hline 90 & $8: 59: 17.23$ & $-47: 27: 57.10$ & 18.0 & 15.9 & $1.89 \pm 0.82$ & $2.43 \pm 1.99$ & $2.11 \mathrm{E}-15$ & $6.16 \mathrm{E}-15$ & 0.29 \\
\hline
\end{tabular}


Table 2-Continued

\begin{tabular}{|c|c|c|c|c|c|c|c|c|c|}
\hline $\begin{array}{l}\text { Chandra } \\
\text { ID }\end{array}$ & $\begin{array}{c}\text { RA } \\
(2000)\end{array}$ & $\begin{array}{c}\text { Dec } \\
(2000)\end{array}$ & $\begin{array}{l}\text { Raw } \\
\text { CNT }\end{array}$ & $\begin{array}{l}\mathrm{Net}^{\mathrm{a}} \\
\mathrm{CNT}\end{array}$ & $\begin{array}{c}N_{H} \\
10^{22} \mathrm{~cm}^{-2}\end{array}$ & $\begin{array}{l}\mathrm{kT} \\
\mathrm{keV}\end{array}$ & $\begin{array}{l}\text { Abs. Flux } \\
{[e r s}\end{array}$ & $\begin{array}{l}\text { Tot. UnAbs. Flux } \\
\left.\mathrm{cm}^{-2} s^{-1}\right]\end{array}$ & $\chi^{2}$ \\
\hline 91 & $8: 59: 16.98$ & $-47: 33: 02.73$ & 20.0 & 18.9 & $7.22 \pm 3.21$ & $4.30 \pm 5.94$ & $4.40 \mathrm{E}-15$ & $1.60 \mathrm{E}-14$ & 0.25 \\
\hline 92 & $8: 59: 16.83$ & $-47: 27: 48.99$ & 16.0 & 14.1 & $5.53 \pm 4.13$ & $10.00 \pm \ldots$ & $3.57 \mathrm{E}-15$ & $8.71 \mathrm{E}-15$ & 0.25 \\
\hline 93 & $8: 59: 16.80$ & $-47: 30: 04.13$ & 47.0 & 45.1 & $2.37 \pm 0.77$ & $10.00 \pm \ldots$ & $8.66 \mathrm{E}-15$ & $1.63 \mathrm{E}-14$ & 0.36 \\
\hline 94 & $8: 59: 16.67$ & $-47: 30: 38.52$ & 22.0 & 20.7 & $8.82 \pm 2.65$ & $1.83 \pm 0.48$ & $4.07 \mathrm{E}-15$ & $3.86 \mathrm{E}-14$ & 0.30 \\
\hline 95 & $8: 59: 16.31$ & $-47: 28: 42.01$ & 6.0 & 4.3 & $6.24 \pm 3.19$ & $0.73 \pm 0.25$ & $5.34 \mathrm{E}-16$ & $4.24 \mathrm{E}-14$ & 0.24 \\
\hline 96 & $8: 59: 16.04$ & $-47: 33: 12.76$ & 13.0 & 11.7 & $2.53 \pm 1.03$ & $1.44 \pm 0.62$ & $1.41 \mathrm{E}-15$ & $7.83 \mathrm{E}-15$ & 0.24 \\
\hline 97 & $8: 59: 16.08$ & $-47: 32: 32.70$ & 8.0 & 6.7 & $62.18 \pm 20.23$ & $1.44 \pm 0.29$ & $2.55 \mathrm{E}-15$ & $3.64 \mathrm{E}-13$ & 0.25 \\
\hline 98 & $8: 59: 16.00$ & $-47: 31: 10.31$ & 24.0 & 22.2 & $2.30 \pm 1.15$ & $10.00 \pm \ldots$ & $4.07 \mathrm{E}-15$ & $7.61 \mathrm{E}-15$ & 0.36 \\
\hline 99 & $8: 59: 15.90$ & $-47: 32: 57.96$ & 31.0 & 30.0 & $2.53 \pm 0.66$ & $1.89 \pm 0.69$ & $3.96 \mathrm{E}-15$ & $1.63 \mathrm{E}-14$ & 0.25 \\
\hline 100 & $8: 59: 15.83$ & $-47: 28: 53.27$ & 37.0 & 35.6 & $2.33 \pm 0.54$ & $1.69 \pm 0.54$ & $4.06 \mathrm{E}-15$ & $1.79 \mathrm{E}-14$ & 0.33 \\
\hline 101 & $8: 59: 15.76$ & $-47: 29: 22.30$ & 13.0 & 12.0 & $0.42 \pm 0.41$ & $2.47 \pm 7.14$ & $1.75 \mathrm{E}-15$ & $3.05 \mathrm{E}-15$ & 0.20 \\
\hline 102 & $8: 59: 15.77$ & $-47: 27: 01.68$ & 34.0 & 32.4 & $3.75 \pm 1.09$ & $3.28 \pm 1.65$ & $6.05 \mathrm{E}-15$ & $1.93 \mathrm{E}-14$ & 0.31 \\
\hline 103 & $8: 59: 15.70$ & $-47: 30: 09.15$ & 14.0 & 12.4 & $15.85 \pm 7.80$ & $3.71 \pm 2.74$ & $3.76 \mathrm{E}-15$ & $2.33 \mathrm{E}-14$ & 0.25 \\
\hline 104 & $8: 59: 15.66$ & $-47: 29: 39.49$ & 6.0 & 4.6 & $13.87 \pm 5.15$ & $0.71 \pm 0.13$ & $8.27 \mathrm{E}-16$ & $2.64 \mathrm{E}-13$ & 0.20 \\
\hline 105 & $8: 59: 15.63$ & $-47: 26: 09.43$ & 18.0 & 13.6 & $1.39 \pm 0.54$ & $1.12 \pm 0.53$ & $1.26 \mathrm{E}-15$ & $6.54 \mathrm{E}-15$ & 0.46 \\
\hline 106 & $8: 59: 15.59$ & $-47: 27: 46.03$ & 27.0 & 25.4 & $0.88 \pm 0.37$ & $2.10 \pm 1.66$ & $2.37 \mathrm{E}-15$ & $5.62 \mathrm{E}-15$ & 0.33 \\
\hline 107 & $8: 59: 14.89$ & $-47: 29: 28.02$ & 32.0 & 30.4 & $2.93 \pm 0.57$ & $1.14 \pm 0.22$ & $3.25 \mathrm{E}-15$ & $2.94 \mathrm{E}-14$ & 0.26 \\
\hline 108 & $8: 59: 14.88$ & $-47: 26: 58.55$ & 52.0 & 49.9 & $17.27 \pm 2.79$ & $1.83 \pm 0.24$ & $1.28 \mathrm{E}-14$ & $2.12 \mathrm{E}-13$ & 0.35 \\
\hline 109 & $8: 59: 14.73$ & $-47: 31: 47.68$ & 23.0 & 21.3 & $4.47 \pm 2.16$ & $2.93 \pm 1.53$ & $3.86 \mathrm{E}-15$ & $1.44 \mathrm{E}-14$ & 0.34 \\
\hline 110 & $8: 59: 14.73$ & $-47: 30: 16.06$ & 10.0 & 8.4 & $8.34 \pm 7.74$ & $10.00 \pm \ldots$ & $2.24 \mathrm{E}-15$ & $6.42 \mathrm{E}-15$ & 0.25 \\
\hline 111 & $8: 59: 14.74$ & $-47: 29: 31.86$ & 71.0 & 69.0 & $1.90 \pm 0.39$ & $3.29 \pm 1.19$ & $9.86 \mathrm{E}-15$ & $2.42 \mathrm{E}-14$ & 0.37 \\
\hline 112 & $8: 59: 14.58$ & $-47: 26: 50.13$ & 28.0 & 26.7 & $3.82 \pm 1.76$ & $10.00 \pm \ldots$ & $5.82 \mathrm{E}-15$ & $1.26 \mathrm{E}-14$ & 0.32 \\
\hline 113 & $8: 59: 14.43$ & $-47: 31: 19.64$ & 34.0 & 32.3 & $3.82 \pm 0.82$ & $1.52 \pm 0.32$ & $4.30 \mathrm{E}-15$ & $2.95 \mathrm{E}-14$ & 0.36 \\
\hline 114 & $8: 59: 14.43$ & $-47: 30: 51.06$ & 34.0 & 31.8 & $5.58 \pm 1.21$ & $1.53 \pm 0.29$ & $4.86 \mathrm{E}-15$ & 4.35E-14 & 0.41 \\
\hline 115 & $8: 59: 14.33$ & $-47: 29: 44.56$ & 16.0 & 14.5 & $8.10 \pm 4.20$ & $3.80 \pm 3.46$ & $3.54 \mathrm{E}-15$ & $1.47 \mathrm{E}-14$ & 0.27 \\
\hline 116 & $8: 59: 14.00$ & $-47: 28: 56.52$ & 12.0 & 11.4 & $30.02 \pm 9.07$ & $1.44 \pm 0.26$ & $1.41 \mathrm{E}-14$ & $7.34 \mathrm{E}-13$ & 0.20 \\
\hline 117 & $8: 59: 13.43$ & $-47: 32: 48.06$ & 17.0 & 15.9 & $3.24 \pm 0.79$ & $0.78 \pm 0.18$ & $2.87 \mathrm{E}-15$ & $7.98 \mathrm{E}-14$ & 0.24 \\
\hline 118 & $8: 59: 13.40$ & $-47: 29: 33.26$ & 28.0 & 26.0 & $5.00 \pm 1.68$ & $2.66 \pm 1.00$ & $4.99 \mathrm{E}-15$ & $2.13 \mathrm{E}-14$ & 0.35 \\
\hline 119 & $8: 59: 12.72$ & $-47: 30: 00.13$ & 29.0 & 27.1 & $8.45 \pm 2.17$ & $1.87 \pm 0.42$ & $5.38 \mathrm{E}-15$ & $4.80 \mathrm{E}-14$ & 0.38 \\
\hline 120 & $8: 59: 12.63$ & $-47: 30: 55.35$ & 11.0 & 8.8 & $11.84 \pm 7.64$ & $7.54 \pm \cdots$ & $2.79 \mathrm{E}-15$ & $1.01 \mathrm{E}-14$ & 0.34 \\
\hline 121 & $8: 59: 11.86$ & $-47: 29: 43.63$ & 21.0 & 19.0 & $4.76 \pm 2.84$ & $7.26 \pm \cdots$ & $4.34 \mathrm{E}-15$ & $1.08 \mathrm{E}-14$ & 0.35 \\
\hline 122 & $8: 59: 11.71$ & $-47: 28: 10.89$ & 12.0 & 10.8 & $0.63 \pm 0.23$ & $0.56 \pm 0.21$ & $5.71 \mathrm{E}-16$ & $3.84 \mathrm{E}-15$ & 0.23 \\
\hline 123 & $8: 59: 11.80$ & $-47: 27: 04.58$ & 8.0 & 6.3 & $0.00 \pm 0.13$ & $2.51 \pm \cdots$ & $5.27 \mathrm{E}-16$ & $5.27 \mathrm{E}-16$ & 0.28 \\
\hline 124 & $8: 59: 11.59$ & $-47: 30: 16.19$ & 22.0 & 19.9 & $32.98 \pm 6.39$ & $1.34 \pm 0.17$ & $5.80 \mathrm{E}-15$ & $4.18 \mathrm{E}-13$ & 0.36 \\
\hline 125 & 8:59:11.09 & $-47: 31: 23.47$ & 10.0 & 8.0 & $17.55 \pm 7.12$ & $1.50 \pm 0.40$ & $1.99 \mathrm{E}-15$ & $5.14 \mathrm{E}-14$ & 0.28 \\
\hline 126 & $8: 59: 10.43$ & $-47: 29: 42.54$ & 12.0 & 9.0 & $2.57 \pm 1.56$ & $1.83 \pm 1.58$ & $1.24 \mathrm{E}-15$ & $5.33 \mathrm{E}-15$ & 0.35 \\
\hline 127 & $8: 59: 10.25$ & $-47: 31: 08.10$ & 19.0 & 17.5 & $21.75 \pm 9.04$ & $10.00 \pm \cdots$ & $6.35 \mathrm{E}-15$ & $3.04 \mathrm{E}-14$ & 0.55 \\
\hline 128 & $8: 59: 10.05$ & $-47: 28: 24.03$ & 5.0 & 4.3 & $3.40 \pm 1.06$ & $0.30 \pm 0.06$ & $1.09 \mathrm{E}-15$ & $5.58 \mathrm{E}-13$ & 0.15 \\
\hline 129 & 8:59:09.94 & $-47: 30: 27.90$ & 34.0 & 32.5 & $9.31 \pm 2.59$ & $2.71 \pm 0.75$ & $7.40 \mathrm{E}-15$ & $4.41 \mathrm{E}-14$ & 0.59 \\
\hline 130 & $8: 59: 10.00$ & $-47: 29: 41.17$ & 23.0 & 20.5 & $1.89 \pm 0.57$ & $1.45 \pm 0.54$ & $2.31 \mathrm{E}-15$ & $1.06 \mathrm{E}-14$ & 0.36 \\
\hline 131 & 8:59:09.72 & $-47: 31: 31.01$ & 17.0 & 15.5 & $1.30 \pm 0.96$ & $10.00 \mathrm{v} \quad \cdots$ & $2.21 \mathrm{E}-15$ & $3.62 \mathrm{E}-15$ & 0.55 \\
\hline 132 & $8: 59: 09.70$ & $-47: 28: 45.58$ & 20.0 & 18.2 & $4.33 \pm 1.33$ & $1.58 \pm 0.48$ & $2.78 \mathrm{E}-15$ & $1.98 \mathrm{E}-14$ & 0.31 \\
\hline 133 & $8: 59: 09.62$ & $-47: 30: 25.60$ & 293.0 & 291.5 & $3.32 \pm 0.31$ & $3.91 \pm 0.59$ & $5.31 \mathrm{E}-14$ & $1.46 \mathrm{E}-13$ & 0.82 \\
\hline 134 & $8: 59: 09.53$ & $-47: 31: 18.46$ & 33.0 & 31.5 & $21.10 \pm 5.66$ & $4.70 \pm 2.50$ & $1.03 \mathrm{E}-14$ & $6.54 \mathrm{E}-14$ & 0.60 \\
\hline 135 & $8: 59: 09.43$ & $-47: 30: 20.16$ & 20.0 & 18.5 & $5.46 \pm 1.97$ & $2.57 \pm 1.14$ & $3.59 \mathrm{E}-15$ & $1.66 \mathrm{E}-14$ & 0.55 \\
\hline
\end{tabular}


Table 2-Continued

\begin{tabular}{|c|c|c|c|c|c|c|c|c|c|}
\hline $\begin{array}{l}\text { Chandra } \\
\text { ID }\end{array}$ & $\begin{array}{c}\mathrm{RA} \\
(2000)\end{array}$ & $\begin{array}{c}\text { Dec } \\
(2000)\end{array}$ & $\begin{array}{l}\text { Raw } \\
\text { CNT }\end{array}$ & $\begin{array}{l}\text { Net }^{a} \\
\text { CNT }\end{array}$ & $\begin{array}{c}N_{H} \\
10^{22} \mathrm{~cm}^{-2}\end{array}$ & $\begin{array}{l}\mathrm{kT} \\
\mathrm{keV}\end{array}$ & $\begin{array}{l}\text { Abs. Flux } \\
{[e r .}\end{array}$ & $\begin{array}{l}\text { Tot. UnAbs. Flux } \\
\left.\mathrm{cm}^{-2} s^{-1}\right]\end{array}$ & $\chi^{2}$ \\
\hline 136 & $8: 59: 09.30$ & $-47: 29: 19.06$ & 27.0 & 24.0 & $2.85 \pm 0.96$ & $1.88 \pm 0.77$ & $3.28 \mathrm{E}-15$ & $1.45 \mathrm{E}-14$ & 0.43 \\
\hline 137 & 8:59:09.14 & $-47: 34: 05.36$ & 506.0 & 502.6 & $0.93 \pm 0.10$ & $6.40 \pm 2.24$ & $7.14 \mathrm{E}-14$ & $1.17 \mathrm{E}-13$ & 0.77 \\
\hline 138 & $8: 59: 09.10$ & $-47: 31: 00.59$ & 15.0 & 13.5 & $6.59 \pm 1.62$ & $0.73 \pm 0.12$ & $1.60 \mathrm{E}-15$ & $1.39 \mathrm{E}-13$ & 0.52 \\
\hline 139 & 8:59:09.08 & $-47: 29: 16.82$ & 27.0 & 24.0 & $0.86 \pm 0.44$ & $3.77 \pm 14.55$ & $2.99 \mathrm{E}-15$ & $5.46 \mathrm{E}-15$ & 0.41 \\
\hline 140 & $8: 59: 09.06$ & $-47: 30: 41.50$ & 43.0 & 41.5 & $2.87 \pm 0.62$ & $1.90 \pm 0.53$ & $5.63 \mathrm{E}-15$ & $2.47 \mathrm{E}-14$ & 0.60 \\
\hline 141 & 8:59:09.06 & $-47: 27: 50.95$ & 9.0 & 7.6 & $0.23 \pm 0.43$ & $1.36 \pm \cdots$ & $4.22 \mathrm{E}-16$ & 7.33E-16 & 0.23 \\
\hline 142 & $8: 59: 08.75$ & $-47: 30: 37.34$ & 290.0 & 288.5 & $3.56 \pm 0.30$ & $2.76 \pm 0.30$ & $4.85 \mathrm{E}-14$ & $1.71 \mathrm{E}-13$ & 0.84 \\
\hline 143 & $8: 59: 08.75$ & $-47: 30: 27.08$ & 79.0 & 77.5 & $6.89 \pm 1.36$ & $5.29 \pm 2.51$ & $1.89 \mathrm{E}-14$ & $6.07 \mathrm{E}-14$ & 0.69 \\
\hline 144 & $8: 59: 08.68$ & $-47: 30: 59.32$ & 20.0 & 18.5 & $1.68 \pm 0.69$ & $2.39 \pm 1.77$ & $2.24 \mathrm{E}-15$ & $6.31 \mathrm{E}-15$ & 0.54 \\
\hline 145 & $8: 59: 08.55$ & $-47: 30: 49.73$ & 82.0 & 80.5 & $3.44 \pm 0.49$ & $1.81 \pm 0.30$ & $1.13 \mathrm{E}-14$ & $5.80 \mathrm{E}-14$ & 0.67 \\
\hline 146 & $8: 59: 08.38$ & $-47: 29: 54.95$ & 121.0 & 117.6 & $1.10 \pm 0.21$ & $2.80 \pm 0.77$ & $1.33 \mathrm{E}-14$ & $2.94 \mathrm{E}-14$ & 0.59 \\
\hline 147 & $8: 59: 08.29$ & $-47: 35: 16.39$ & 36.0 & 32.2 & $1.45 \pm 0.47$ & $2.60 \pm 1.39$ & $4.37 \mathrm{E}-15$ & $1.11 \mathrm{E}-14$ & 0.43 \\
\hline 148 & $8: 59: 08.08$ & $-47: 30: 47.60$ & 87.0 & 85.5 & $3.35 \pm 0.49$ & $2.14 \pm 0.40$ & $1.28 \mathrm{E}-14$ & $5.43 \mathrm{E}-14$ & 0.67 \\
\hline 149 & 8:59:08.04 & $-47: 30: 38.77$ & 74.0 & 72.5 & $3.45 \pm 0.57$ & $2.08 \pm 0.42$ & $1.07 \mathrm{E}-14$ & $4.74 \mathrm{E}-14$ & 0.67 \\
\hline 150 & $8: 59: 07.96$ & $-47: 31: 22.42$ & 22.0 & 20.5 & $8.23 \pm 3.08$ & $3.80 \pm 2.83$ & $4.85 \mathrm{E}-15$ & $2.02 \mathrm{E}-14$ & 0.56 \\
\hline 151 & $8: 59: 07.97$ & $-47: 30: 25.94$ & 73.0 & 71.5 & $3.53 \pm 0.68$ & $3.71 \pm 1.23$ & $1.40 \mathrm{E}-14$ & $4.06 \mathrm{E}-14$ & 0.68 \\
\hline 152 & $8: 59: 07.91$ & $-47: 31: 00.83$ & 126.0 & 124.5 & $2.76 \pm 0.43$ & $4.53 \pm 1.52$ & $2.21 \mathrm{E}-14$ & $5.32 \mathrm{E}-14$ & 0.75 \\
\hline 153 & $8: 59: 07.85$ & $-47: 31: 36.63$ & 13.0 & 10.9 & $8.51 \pm 5.54$ & $10.00 \pm \ldots$ & $2.96 \mathrm{E}-15$ & $8.56 \mathrm{E}-15$ & 0.27 \\
\hline 154 & $8: 59: 07.85$ & $-47: 31: 12.15$ & 21.0 & 19.5 & $7.29 \pm 3.52$ & $4.82 \pm 6.59$ & $4.84 \mathrm{E}-15$ & $1.66 \mathrm{E}-14$ & 0.56 \\
\hline 155 & $8: 59: 07.73$ & $-47: 30: 44.75$ & 22.0 & 20.5 & $3.69 \pm 1.16$ & $1.83 \pm 0.72$ & $2.93 \mathrm{E}-15$ & $1.54 \mathrm{E}-14$ & 0.55 \\
\hline 156 & $8: 59: 07.44$ & $-47: 29: 16.12$ & 45.0 & 42.7 & $3.20 \pm 0.96$ & $3.79 \pm 2.01$ & $7.87 \mathrm{E}-15$ & $2.17 \mathrm{E}-14$ & 0.44 \\
\hline 157 & $8: 59: 07.49$ & $-47: 25: 38.88$ & 11.0 & 6.8 & $0.00 \pm 0.13$ & $1.83 \pm 9.97$ & $7.81 \mathrm{E}-16$ & $7.81 \mathrm{E}-16$ & 0.34 \\
\hline 158 & $8: 59: 07.37$ & $-47: 30: 46.17$ & 39.0 & 37.5 & $1.60 \pm 0.49$ & $2.79 \pm 1.36$ & $4.84 \mathrm{E}-15$ & $1.22 \mathrm{E}-14$ & 0.59 \\
\hline 159 & $8: 59: 07.35$ & $-47: 33: 05.44$ & 258.0 & 256.3 & $1.17 \pm 0.13$ & $2.51 \pm 0.37$ & $2.85 \mathrm{E}-14$ & $6.81 \mathrm{E}-14$ & 0.60 \\
\hline 160 & $8: 59: 07.33$ & $-47: 31: 36.08$ & 26.0 & 23.8 & $1.06 \pm 0.59$ & $10.00 \pm \ldots$ & $3.69 \mathrm{E}-15$ & $5.80 \mathrm{E}-15$ & 0.28 \\
\hline 161 & $8: 59: 07.26$ & $-47: 31: 14.88$ & 61.0 & 59.5 & $6.19 \pm 1.18$ & $2.58 \pm 0.56$ & $1.17 \mathrm{E}-14$ & $5.77 \mathrm{E}-14$ & 0.67 \\
\hline 162 & 8:59:07.19 & $-47: 29: 57.97$ & 47.0 & 45.5 & $18.72 \pm 2.68$ & $1.20 \pm 0.10$ & $9.45 \mathrm{E}-15$ & $4.94 \mathrm{E}-13$ & 0.62 \\
\hline 163 & $8: 59: 07.16$ & $-47: 31: 18.01$ & 103.0 & 101.5 & $5.94 \pm 0.92$ & $3.10 \pm 0.57$ & $2.10 \mathrm{E}-14$ & $8.64 \mathrm{E}-14$ & 0.71 \\
\hline 164 & $8: 59: 06.98$ & $-47: 30: 32.73$ & 14.0 & 12.5 & $3.98 \pm 1.29$ & $1.23 \pm 0.40$ & $1.70 \mathrm{E}-15$ & $1.71 \mathrm{E}-14$ & 0.52 \\
\hline 165 & $8: 59: 06.95$ & $-47: 30: 06.22$ & 12.0 & 10.5 & $3.15 \pm 1.72$ & $2.72 \pm 2.97$ & $1.72 \mathrm{E}-15$ & $5.79 \mathrm{E}-15$ & 0.52 \\
\hline 166 & 8:59:06.91 & $-47: 31: 02.53$ & 216.0 & 214.5 & $5.83 \pm 0.66$ & $5.05 \pm 1.10$ & $4.94 \mathrm{E}-14$ & $1.50 \mathrm{E}-13$ & 0.80 \\
\hline 167 & $8: 59: 06.82$ & $-47: 29: 41.39$ & 87.0 & 82.7 & $3.95 \pm 0.88$ & $5.96 \pm 4.77$ & $1.78 \mathrm{E}-14$ & $4.38 \mathrm{E}-14$ & 0.65 \\
\hline 168 & $8: 59: 06.72$ & $-47: 29: 57.96$ & 45.0 & 43.5 & $2.62 \pm 0.87$ & $10.00 \pm \ldots$ & $8.80 \mathrm{E}-15$ & $1.70 \mathrm{E}-14$ & 0.63 \\
\hline 169 & $8: 59: 06.60$ & $-47: 31: 00.48$ & 80.0 & 78.5 & $5.29 \pm 1.00$ & $4.34 \pm 1.49$ & $1.72 \mathrm{E}-14$ & $5.41 \mathrm{E}-14$ & 0.68 \\
\hline 170 & $8: 59: 06.63$ & $-47: 30: 22.13$ & 280.0 & 278.5 & $23.62 \pm 1.89$ & $10.00 \pm \cdots$ & $1.17 \mathrm{E}-13$ & $5.92 \mathrm{E}-13$ & 0.88 \\
\hline 171 & $8: 59: 06.62$ & $-47: 30: 18.58$ & 69.0 & 67.5 & $2.77 \pm 0.70$ & $7.68 \pm 54.54$ & $1.33 \mathrm{E}-14$ & $2.75 \mathrm{E}-14$ & 0.68 \\
\hline 172 & $8: 59: 06.55$ & $-47: 31: 15.90$ & 30.0 & 28.5 & $5.70 \pm 2.19$ & $10.00 \pm \cdots$ & $6.98 \mathrm{E}-15$ & $1.72 \mathrm{E}-14$ & 0.59 \\
\hline 173 & $8: 59: 06.51$ & $-47: 31: 26.33$ & 31.0 & 29.5 & $20.60 \pm 3.32$ & $0.88 \pm 0.06$ & $5.30 \mathrm{E}-15$ & $1.10 \mathrm{E}-12$ & 0.57 \\
\hline 174 & $8: 59: 06.44$ & $-47: 30: 37.96$ & 128.0 & 126.5 & $1.67 \pm 0.26$ & $3.26 \pm 0.85$ & $1.81 \mathrm{E}-14$ & $4.28 \mathrm{E}-14$ & 0.73 \\
\hline 175 & $8: 59: 06.39$ & $-47: 30: 03.82$ & 10.0 & 8.5 & $5.08 \pm 4.08$ & $4.70 \pm \ldots$ & $1.99 \mathrm{E}-15$ & $5.88 \mathrm{E}-15$ & 0.51 \\
\hline 176 & $8: 59: 06.36$ & $-47: 29: 10.16$ & 53.0 & 50.8 & $2.17 \pm 0.59$ & $3.71 \pm 2.01$ & $9.14 \mathrm{E}-15$ & $2.21 \mathrm{E}-14$ & 0.45 \\
\hline 177 & $8: 59: 06.33$ & $-47: 31: 07.78$ & 19.0 & 17.5 & $3.13 \pm 1.73$ & $10.00 \pm \ldots$ & $3.66 \mathrm{E}-15$ & $7.44 \mathrm{E}-15$ & 0.55 \\
\hline 178 & $8: 59: 06.25$ & $-47: 30: 26.08$ & 208.0 & 206.5 & $1.68 \pm 0.22$ & $4.20 \pm 1.09$ & $3.47 \mathrm{E}-14$ & 7.37E-14 & 0.81 \\
\hline 179 & $8: 59: 06.26$ & $-47: 25: 31.09$ & 34.0 & 29.6 & $2.62 \pm 1.10$ & $10.00 \pm \ldots$ & $6.46 \mathrm{E}-15$ & $1.25 \mathrm{E}-14$ & 0.51 \\
\hline 180 & $8: 59: 06.19$ & $-47: 30: 44.71$ & 62.0 & 60.5 & $1.86 \pm 0.32$ & $1.36 \pm 0.27$ & $6.09 \mathrm{E}-15$ & $3.00 \mathrm{E}-14$ & 0.63 \\
\hline
\end{tabular}


Table 2-Continued

\begin{tabular}{|c|c|c|c|c|c|c|c|c|c|}
\hline $\begin{array}{l}\text { Chandra } \\
\text { ID }\end{array}$ & $\begin{array}{c}\text { RA } \\
(2000)\end{array}$ & $\begin{array}{c}\text { Dec } \\
(2000)\end{array}$ & $\begin{array}{l}\text { Raw } \\
\text { CNT }\end{array}$ & $\begin{array}{l}\text { Net }^{a} \\
\text { CNT }\end{array}$ & $\begin{array}{c}N_{H} \\
10^{22} \mathrm{~cm}^{-2}\end{array}$ & $\begin{array}{l}\mathrm{kT} \\
\mathrm{keV}\end{array}$ & \multicolumn{2}{|c|}{$\left[\mathrm{ergcm}^{-2} \mathrm{~s}^{-1}\right]$} & $\chi^{2}$ \\
\hline 181 & $8: 59: 06.23$ & $-47: 29: 58.75$ & 36.0 & 34.5 & $2.53 \pm 0.58$ & $1.65 \pm 0.47$ & $4.24 \mathrm{E}-15$ & $2.01 \mathrm{E}-14$ & 0.58 \\
\hline 182 & 8:59:06.19 & $-47: 30: 51.86$ & 45.0 & 43.5 & $2.32 \pm 0.67$ & $2.92 \pm 1.27$ & $7.09 \mathrm{E}-15$ & $2.00 \mathrm{E}-14$ & 0.63 \\
\hline 183 & 8:59:06.11 & $-47: 31: 01.33$ & 57.0 & 55.5 & $3.93 \pm 1.02$ & $9.00 \pm$ & $1.25 \mathrm{E}-14$ & $2.78 \mathrm{E}-14$ & 0.65 \\
\hline 184 & $8: 59: 06.14$ & $-47: 30: 35.38$ & 337.0 & 335.5 & $1.71 \pm 0.17$ & $4.20 \pm 0.78$ & $5.25 \mathrm{E}-14$ & $1.12 \mathrm{E}-13$ & 0.85 \\
\hline 185 & 8:59:06.03 & $-47: 31: 34.09$ & 17.0 & 13.6 & $13.24 \pm 4.34$ & $1.25 \pm 0.23$ & $2.91 \mathrm{E}-15$ & $9.02 \mathrm{E}-14$ & 0.35 \\
\hline 186 & 8:59:06.02 & $-47: 30: 21.00$ & 25.0 & 23.5 & $2.45 \pm 0.75$ & $1.82 \pm 0.78$ & $2.88 \mathrm{E}-15$ & $1.20 \mathrm{E}-14$ & 0.56 \\
\hline 187 & 8:59:05.95 & $-47: 35: 19.58$ & 138.0 & 133.6 & $2.65 \pm 0.50$ & $10.00 \pm \ldots$ & $2.88 \mathrm{E}-14$ & $5.59 \mathrm{E}-14$ & 0.68 \\
\hline 188 & 8:59:05.94 & $-47: 31: 51.62$ & 27.0 & 25.1 & $6.94 \pm 3.25$ & $10.00 \pm \ldots$ & $6.65 \mathrm{E}-15$ & $1.77 \mathrm{E}-14$ & 0.32 \\
\hline 189 & 8:59:05.95 & $-47: 30: 55.03$ & 44.0 & 42.5 & $1.11 \pm 0.46$ & $10.00 \pm 51.23$ & $7.22 \mathrm{E}-15$ & $1.15 \mathrm{E}-14$ & 0.62 \\
\hline 190 & 8:59:05.85 & $-47: 30: 36.81$ & 262.0 & 260.5 & $1.77 \pm 0.24$ & $10.00 \pm \ldots$ & $4.85 \mathrm{E}-14$ & $8.50 \mathrm{E}-14$ & 0.89 \\
\hline 191 & $8: 59: 05.88$ & $-47: 30: 30.92$ & 297.0 & 295.5 & $1.73 \pm 0.17$ & $3.28 \pm 0.49$ & $4.67 \mathrm{E}-14$ & $1.11 \mathrm{E}-13$ & 0.81 \\
\hline 192 & $8: 59: 05.70$ & $-47: 34: 47.63$ & 4.0 & 0.4 & $\ldots$ & $\ldots$ & $\ldots$ & $\ldots$ & $\ldots$ \\
\hline 193 & $8: 59: 05.75$ & $-47: 30: 09.17$ & 403.0 & 401.5 & $1.78 \pm 0.16$ & $4.45 \pm 0.82$ & $6.23 \mathrm{E}-14$ & $1.32 \mathrm{E}-13$ & 0.88 \\
\hline 194 & $8: 59: 05.67$ & $-47: 30: 46.72$ & 293.0 & 291.5 & $1.59 \pm 0.17$ & $3.31 \pm 0.53$ & $4.11 \mathrm{E}-14$ & $9.47 \mathrm{E}-14$ & 0.84 \\
\hline 195 & 8:59:05.65 & $-47: 30: 41.16$ & 6767.0 & 6765.5 & $1.57 \pm 0.03$ & $1.53 \pm 0.03$ & $6.79 \mathrm{E}-13$ & $2.64 \mathrm{E}-12$ & 1.18 \\
\hline 196 & 8:59:05.52 & $-47: 30: 35.98$ & 931.0 & 929.5 & $1.69 \pm 0.09$ & $3.18 \pm 0.26$ & $1.41 \mathrm{E}-13$ & $3.39 \mathrm{E}-13$ & 0.93 \\
\hline 197 & 8:59:05.45 & $-47: 30: 30.49$ & 315.0 & 313.5 & $1.34 \pm 0.13$ & $2.49 \pm 0.33$ & $3.61 \mathrm{E}-14$ & $9.10 \mathrm{E}-14$ & 0.81 \\
\hline 198 & $8: 59: 05.44$ & $-47: 30: 48.50$ & 124.0 & 122.5 & $1.51 \pm 0.25$ & $3.21 \pm 0.86$ & $1.69 \mathrm{E}-14$ & $3.90 \mathrm{E}-14$ & 0.72 \\
\hline 199 & 8:59:05.40 & $-47: 30: 03.03$ & 79.0 & 77.5 & $2.90 \pm 0.46$ & $2.08 \pm 0.43$ & $1.11 \mathrm{E}-14$ & $4.47 \mathrm{E}-14$ & 0.67 \\
\hline 200 & $8: 59: 05.21$ & $-47: 30: 17.43$ & 54.0 & 52.5 & $0.88 \pm 0.31$ & $6.07 \pm 29.52$ & $7.25 \mathrm{E}-15$ & $1.18 \mathrm{E}-14$ & 0.62 \\
\hline 201 & $8: 59: 05.15$ & $-47: 29: 53.26$ & 39.0 & 35.2 & $2.20 \pm 0.86$ & $4.12 \pm 4.28$ & $5.85 \mathrm{E}-15$ & $1.36 \mathrm{E}-14$ & 0.45 \\
\hline 202 & $8: 59: 05.12$ & $-47: 31: 01.58$ & 84.0 & 82.5 & $1.98 \pm 0.40$ & $3.42 \pm 1.16$ & $1.28 \mathrm{E}-14$ & $3.13 \mathrm{E}-14$ & 0.71 \\
\hline 203 & $8: 59: 05.06$ & $-47: 33: 07.90$ & 18.0 & 16.4 & $0.00 \pm 0.07$ & $1.23 \pm 9.72$ & $1.09 \mathrm{E}-15$ & $1.09 \mathrm{E}-15$ & 0.30 \\
\hline 204 & 8:59:05.05 & $-47: 30: 47.82$ & 166.0 & 164.5 & $1.52 \pm 0.21$ & $3.35 \pm 0.80$ & $2.32 \mathrm{E}-14$ & $5.26 \mathrm{E}-14$ & 0.75 \\
\hline 205 & 8:59:05.01 & $-47: 31: 26.86$ & 29.0 & 27.5 & $4.16 \pm 1.07$ & $1.60 \pm 0.44$ & $3.70 \mathrm{E}-15$ & $2.49 \mathrm{E}-14$ & 0.58 \\
\hline 206 & 8:59:05.04 & $-47: 30: 58.43$ & 151.0 & 149.5 & $4.10 \pm 0.45$ & $2.14 \pm 0.27$ & $2.53 \mathrm{E}-14$ & $1.20 \mathrm{E}-13$ & 0.75 \\
\hline 207 & 8:59:04.91 & $-47: 32: 41.40$ & 39.0 & 37.5 & $1.82 \pm 0.76$ & $4.68 \pm 9.74$ & $6.03 \mathrm{E}-15$ & $1.26 \mathrm{E}-14$ & 0.37 \\
\hline 208 & $8: 59: 04.84$ & $-47: 30: 55.01$ & 75.0 & 73.5 & $2.17 \pm 0.40$ & $2.75 \pm 0.80$ & $1.09 \mathrm{E}-14$ & $3.10 \mathrm{E}-14$ & 0.68 \\
\hline 209 & 8:59:04.79 & $-47: 30: 36.75$ & 122.0 & 120.5 & $1.54 \pm 0.23$ & $2.32 \pm 0.49$ & $1.42 \mathrm{E}-14$ & $3.94 \mathrm{E}-14$ & 0.70 \\
\hline 210 & 8:59:04.72 & $-47: 28: 33.45$ & 13.0 & 11.0 & $6.94 \pm 2.02$ & $0.74 \pm 0.15$ & $1.36 \mathrm{E}-15$ & $1.18 \mathrm{E}-13$ & 0.32 \\
\hline 211 & 8:59:04.64 & $-47: 30: 28.24$ & 116.0 & 114.5 & $1.35 \pm 0.28$ & $6.94 \pm 10.26$ & $1.80 \mathrm{E}-14$ & $3.15 \mathrm{E}-14$ & 0.73 \\
\hline 212 & 8:59:04.59 & $-47: 30: 51.05$ & 297.0 & 295.5 & $1.93 \pm 0.20$ & $4.03 \pm 0.72$ & $4.84 \mathrm{E}-14$ & $1.09 \mathrm{E}-13$ & 0.85 \\
\hline 213 & $8: 59: 04.52$ & $-47: 30: 34.94$ & 89.0 & 87.5 & $2.22 \pm 0.28$ & $1.35 \pm 0.19$ & $9.07 \mathrm{E}-15$ & $5.12 \mathrm{E}-14$ & 0.66 \\
\hline 214 & 8:59:04.43 & $-47: 30: 56.45$ & 231.0 & 229.5 & $2.05 \pm 0.25$ & $5.34 \pm 1.79$ & $4.05 \mathrm{E}-14$ & $8.40 \mathrm{E}-14$ & 0.84 \\
\hline 215 & 8:59:04.33 & $-47: 31: 31.15$ & 18.0 & 16.5 & $7.76 \pm 1.94$ & $0.95 \pm 0.16$ & $2.40 \mathrm{E}-15$ & $9.59 \mathrm{E}-14$ & 0.54 \\
\hline 216 & 8:59:04.33 & $-47: 31: 05.90$ & 188.0 & 186.5 & $2.34 \pm 0.29$ & $3.76 \pm 0.82$ & $3.15 \mathrm{E}-14$ & $7.77 \mathrm{E}-14$ & 0.77 \\
\hline 217 & 8:59:04.09 & $-47: 31: 12.16$ & 35.0 & 33.5 & $3.17 \pm 0.87$ & $1.83 \pm 0.50$ & $4.84 \mathrm{E}-15$ & $2.33 \mathrm{E}-14$ & 0.59 \\
\hline 218 & 8:59:04.12 & $-47: 27: 34.90$ & 24.0 & 23.3 & $1.98 \pm 0.88$ & $7.54 \pm \ldots$ & $1.52 \mathrm{E}-14$ & $2.87 \mathrm{E}-14$ & 0.25 \\
\hline 219 & 8:59:04.02 & $-47: 30: 56.03$ & 48.0 & 46.5 & $1.10 \pm 0.41$ & $10.00 \pm \ldots$ & $7.88 \mathrm{E}-15$ & $1.25 \mathrm{E}-14$ & 0.63 \\
\hline 220 & 8:59:04.03 & $-47: 30: 34.80$ & 20.0 & 18.5 & $1.33 \pm 0.51$ & $1.72 \pm 1.03$ & $1.86 \mathrm{E}-15$ & $6.00 \mathrm{E}-15$ & 0.54 \\
\hline 221 & $8: 59: 03.97$ & $-47: 31: 22.21$ & 38.0 & 36.5 & $3.49 \pm 0.80$ & $1.93 \pm 0.55$ & $5.46 \mathrm{E}-15$ & $2.63 \mathrm{E}-14$ & 0.60 \\
\hline 222 & $8: 59: 03.87$ & $-47: 31: 04.90$ & 73.0 & 71.5 & $1.63 \pm 0.33$ & $2.69 \pm 0.86$ & $9.47 \mathrm{E}-15$ & $2.45 \mathrm{E}-14$ & 0.67 \\
\hline 223 & 8:59:03.89 & $-47: 29: 54.35$ & 19.0 & 16.2 & $0.83 \pm 0.62$ & $4.19 \pm \ldots$ & $2.03 \mathrm{E}-15$ & $3.58 \mathrm{E}-15$ & 0.33 \\
\hline 224 & 8:59:03.82 & $-47: 31: 09.26$ & 75.0 & 73.5 & $2.84 \pm 0.53$ & $2.71 \pm 0.68$ & $1.20 \mathrm{E}-14$ & $3.86 \mathrm{E}-14$ & 0.67 \\
\hline 225 & 8:59:03.81 & $-47: 30: 48.54$ & 104.0 & 102.5 & $1.01 \pm 0.20$ & $2.58 \pm 0.74$ & $1.11 \mathrm{E}-14$ & $2.48 \mathrm{E}-14$ & 0.68 \\
\hline
\end{tabular}


Table 2-Continued

\begin{tabular}{|c|c|c|c|c|c|c|c|c|c|}
\hline $\begin{array}{l}\text { Chandra } \\
\text { ID }\end{array}$ & $\begin{array}{c}\mathrm{RA} \\
(2000)\end{array}$ & $\begin{array}{c}\text { Dec } \\
(2000)\end{array}$ & $\begin{array}{l}\text { Raw } \\
\text { CNT }\end{array}$ & $\begin{array}{l}\mathrm{Net}^{\mathrm{a}} \\
\mathrm{CNT}\end{array}$ & $\begin{array}{c}N_{H} \\
10^{22} \mathrm{~cm}^{-2}\end{array}$ & $\begin{array}{l}\mathrm{kT} \\
\mathrm{keV}\end{array}$ & $\begin{array}{r}\text { Abs. Flux } \\
{[e r}\end{array}$ & $\begin{array}{l}\text { Tot. UnAbs. Flux } \\
\left.\mathrm{gcm}^{-2} \mathrm{~s}^{-1}\right]\end{array}$ & $\chi^{2}$ \\
\hline 226 & 8:59:03.81 & $-47: 30: 04.56$ & 29.0 & 27.5 & $3.10 \pm 0.67$ & $1.16 \pm 0.27$ & $2.86 \mathrm{E}-15$ & $2.64 \mathrm{E}-14$ & 0.57 \\
\hline 227 & 8:59:03.74 & $-47: 31: 42.58$ & 33.0 & 30.6 & $8.81 \pm 1.52$ & $1.02 \pm 0.12$ & $4.93 \mathrm{E}-15$ & $1.81 \mathrm{E}-13$ & 0.32 \\
\hline 228 & $8: 59: 03.75$ & $-47: 27: 59.79$ & 33.0 & 31.8 & $0.49 \pm 0.22$ & $1.44 \pm 0.67$ & $2.57 \mathrm{E}-15$ & $5.87 \mathrm{E}-15$ & 0.28 \\
\hline 229 & $8: 59: 03.66$ & $-47: 30: 40.32$ & 510.0 & 508.5 & $2.76 \pm 0.23$ & $9.31 \pm 6.93$ & $1.04 \mathrm{E}-13$ & $2.06 \mathrm{E}-13$ & 0.91 \\
\hline 230 & 8:59:03.61 & $-47: 31: 50.04$ & 61.0 & 58.9 & $5.53 \pm 1.12$ & $2.32 \pm 0.45$ & $1.11 \mathrm{E}-14$ & $5.71 \mathrm{E}-14$ & 0.40 \\
\hline 231 & $8: 59: 03.57$ & $-47: 30: 32.67$ & 105.0 & 103.5 & $1.77 \pm 0.31$ & $3.33 \pm 1.01$ & $1.47 \mathrm{E}-14$ & $3.51 \mathrm{E}-14$ & 0.71 \\
\hline 232 & 8:59:03.51 & $-47: 30: 58.52$ & 511.0 & 509.5 & $1.76 \pm 0.13$ & $3.45 \pm 0.40$ & $7.41 \mathrm{E}-14$ & $1.73 \mathrm{E}-13$ & 0.85 \\
\hline 233 & 8:59:03.36 & $-47: 30: 45.71$ & 154.0 & 152.5 & $1.68 \pm 0.24$ & $2.93 \pm 0.63$ & $2.04 \mathrm{E}-14$ & $5.08 \mathrm{E}-14$ & 0.77 \\
\hline 234 & 8:59:03.34 & $-47: 28: 53.79$ & 36.0 & 33.3 & $2.55 \pm 0.95$ & $7.68 \pm \ldots$ & $6.63 \mathrm{E}-15$ & $1.33 \mathrm{E}-14$ & 0.43 \\
\hline 235 & 8:59:03.10 & $-47: 30: 09.03$ & 20.0 & 18.5 & $1.33 \pm 0.54$ & $1.83 \pm 1.26$ & $1.85 \mathrm{E}-15$ & $5.68 \mathrm{E}-15$ & 0.53 \\
\hline 236 & 8:59:03.07 & $-47: 29: 37.46$ & 161.0 & 158.6 & $2.33 \pm 0.32$ & $4.07 \pm 1.07$ & $2.85 \mathrm{E}-14$ & $6.79 \mathrm{E}-14$ & 0.57 \\
\hline 237 & 8:59:03.05 & $-47: 30: 51.63$ & 44.0 & 42.5 & $2.59 \pm 0.83$ & $10.00 \pm \ldots$ & $8.52 \mathrm{E}-15$ & $1.64 \mathrm{E}-14$ & 0.63 \\
\hline 238 & 8:59:03.03 & $-47: 30: 43.74$ & 168.0 & 166.5 & $1.71 \pm 0.24$ & $4.46 \pm 1.44$ & $2.57 \mathrm{E}-14$ & $5.36 \mathrm{E}-14$ & 0.80 \\
\hline 239 & 8:59:03.00 & $-47: 30: 35.85$ & 54.0 & 52.5 & $1.04 \pm 0.40$ & $9.34 \pm 40.13$ & $8.00 \mathrm{E}-15$ & $1.26 \mathrm{E}-14$ & 0.64 \\
\hline 240 & 8:59:02.96 & $-47: 30: 40.12$ & 59.0 & 57.5 & $2.52 \pm 0.48$ & $1.99 \pm 0.50$ & $7.64 \mathrm{E}-15$ & $2.99 \mathrm{E}-14$ & 0.65 \\
\hline 241 & 8:59:02.92 & $-47: 31: 02.78$ & 24.0 & 22.5 & $0.97 \pm 0.49$ & $4.27 \pm 58.68$ & $2.97 \mathrm{E}-15$ & $5.41 \mathrm{E}-15$ & 0.55 \\
\hline 242 & $8: 59: 02.88$ & $-47: 26: 12.34$ & 16.0 & 11.4 & $0.00 \pm 0.05$ & $1.44 \pm 2.65$ & $9.17 \mathrm{E}-16$ & $9.18 \mathrm{E}-16$ & 0.47 \\
\hline 243 & $8: 59: 02.82$ & $-47: 33: 29.91$ & 7.0 & 1.7 & $0.00 \pm 0.07$ & $0.07 \pm 0.01$ & $7.10 \mathrm{E}-15$ & $7.12 \mathrm{E}-15$ & 0.45 \\
\hline 244 & $8: 59: 02.80$ & $-47: 31: 13.34$ & 20.0 & 18.5 & $3.09 \pm 0.99$ & $1.83 \pm 0.79$ & $2.79 \mathrm{E}-15$ & $1.32 \mathrm{E}-14$ & 0.54 \\
\hline 245 & $8: 59: 02.67$ & $-47: 31: 37.18$ & 81.0 & 78.3 & $1.92 \pm 0.47$ & $10.00 \pm \cdots$ & $1.52 \mathrm{E}-14$ & $2.72 \mathrm{E}-14$ & 0.49 \\
\hline 246 & $8: 59: 02.61$ & $-47: 28: 46.52$ & 47.0 & 44.7 & $1.88 \pm 0.43$ & $2.11 \pm 0.69$ & $5.67 \mathrm{E}-15$ & $1.84 \mathrm{E}-14$ & 0.42 \\
\hline 247 & $8: 59: 02.53$ & $-47: 31: 47.66$ & 188.0 & 184.5 & $2.08 \pm 0.33$ & $10.00 \pm \cdots$ & $3.47 \mathrm{E}-14$ & $6.33 \mathrm{E}-14$ & 0.65 \\
\hline 248 & $8: 59: 02.56$ & $-47: 30: 46.54$ & 91.0 & 89.5 & $2.49 \pm 0.41$ & $2.48 \pm 0.53$ & $1.30 \mathrm{E}-14$ & $4.22 \mathrm{E}-14$ & 0.69 \\
\hline 249 & $8: 59: 02.47$ & $-47: 30: 53.04$ & 105.0 & 103.5 & $2.08 \pm 0.39$ & $5.16 \pm 3.43$ & $1.75 \mathrm{E}-14$ & $3.69 \mathrm{E}-14$ & 0.73 \\
\hline 250 & $8: 59: 02.40$ & $-47: 31: 10.22$ & 31.0 & 29.5 & $1.17 \pm 0.57$ & $10.00 \pm \ldots$ & $4.78 \mathrm{E}-15$ & $7.67 \mathrm{E}-15$ & 0.59 \\
\hline 251 & $8: 59: 02.41$ & $-47: 29: 47.87$ & 34.0 & 31.6 & $2.34 \pm 0.65$ & $2.31 \pm 1.00$ & $4.66 \mathrm{E}-15$ & $1.55 \mathrm{E}-14$ & 0.35 \\
\hline 252 & $8: 59: 02.37$ & $-47: 29: 57.83$ & 149.0 & 147.5 & $2.22 \pm 0.30$ & $3.15 \pm 0.63$ & $2.45 \mathrm{E}-14$ & $6.51 \mathrm{E}-14$ & 0.76 \\
\hline 253 & $8: 59: 02.16$ & $-47: 30: 16.83$ & 1094.0 & 1092.5 & $1.74 \pm 0.09$ & $3.35 \pm 0.25$ & $1.57 \mathrm{E}-13$ & $3.70 \mathrm{E}-13$ & 0.92 \\
\hline 254 & $8: 59: 02.08$ & $-47: 32: 09.81$ & 303.0 & 300.6 & $4.11 \pm 0.43$ & $10.00 \pm 28.25$ & $6.97 \mathrm{E}-14$ & $1.54 \mathrm{E}-13$ & 0.65 \\
\hline 255 & 8:59:01.97 & $-47: 31: 02.46$ & 190.0 & 188.5 & $1.94 \pm 0.22$ & $2.78 \pm 0.48$ & $2.61 \mathrm{E}-14$ & $7.10 \mathrm{E}-14$ & 0.76 \\
\hline 256 & 8:59:01.95 & $-47: 31: 15.20$ & 190.0 & 188.5 & $1.54 \pm 0.24$ & $10.00 \pm 19.94$ & $3.29 \mathrm{E}-14$ & $5.58 \mathrm{E}-14$ & 0.80 \\
\hline 257 & 8:59:01.94 & $-47: 30: 35.73$ & 46.0 & 44.5 & $1.05 \pm 0.40$ & $7.25 \pm \ldots$ & $6.70 \mathrm{E}-15$ & $1.10 \mathrm{E}-14$ & 0.63 \\
\hline 258 & $8: 59: 01.87$ & $-47: 30: 57.24$ & 161.0 & 159.5 & $1.03 \pm 0.20$ & $7.71 \pm 12.21$ & $2.40 \mathrm{E}-14$ & $3.89 \mathrm{E}-14$ & 0.79 \\
\hline 259 & 8:59:01.73 & $-47: 30: 46.36$ & 87.0 & 85.5 & $1.13 \pm 0.25$ & $3.65 \pm 1.53$ & $1.10 \mathrm{E}-14$ & $2.19 \mathrm{E}-14$ & 0.69 \\
\hline 260 & $8: 59: 01.65$ & $-47: 32: 11.56$ & 20.0 & 17.5 & $2.84 \pm 0.73$ & $0.90 \pm 0.18$ & $1.77 \mathrm{E}-15$ & $2.76 \mathrm{E}-14$ & 0.32 \\
\hline 261 & $8: 59: 01.67$ & $-47: 30: 37.41$ & 110.0 & 108.5 & $1.09 \pm 0.21$ & $3.04 \pm 0.95$ & $1.29 \mathrm{E}-14$ & $2.74 \mathrm{E}-14$ & 0.70 \\
\hline 262 & $8: 59: 01.57$ & $-47: 31: 19.52$ & 122.0 & 120.5 & $2.38 \pm 0.34$ & $2.72 \pm 0.58$ & $1.77 \mathrm{E}-14$ & $5.29 \mathrm{E}-14$ & 0.74 \\
\hline 263 & $8: 59: 01.56$ & $-47: 31: 10.27$ & 216.0 & 214.5 & $2.31 \pm 0.24$ & $2.48 \pm 0.34$ & $3.02 \mathrm{E}-14$ & $9.50 \mathrm{E}-14$ & 0.84 \\
\hline 264 & 8:59:01.21 & $-47: 31: 32.43$ & 24.0 & 20.9 & $3.23 \pm 1.06$ & $1.86 \pm 0.78$ & $3.19 \mathrm{E}-15$ & $1.53 \mathrm{E}-14$ & 0.41 \\
\hline 265 & 8:59:01.12 & $-47: 32: 42.13$ & 21.0 & 15.3 & $4.15 \pm 2.01$ & $2.32 \pm 1.33$ & $2.81 \mathrm{E}-15$ & $1.23 \mathrm{E}-14$ & 0.46 \\
\hline 266 & 8:59:01.13 & $-47: 30: 00.81$ & 9.0 & 7.5 & $0.00 \pm 0.11$ & $1.83 \pm 6.41$ & $7.24 \mathrm{E}-16$ & $7.24 \mathrm{E}-16$ & 0.49 \\
\hline 267 & 8:59:01.12 & $-47: 30: 08.98$ & 24.0 & 22.5 & $11.95 \pm 2.25$ & $0.76 \pm 0.08$ & $3.75 \mathrm{E}-15$ & $6.63 \mathrm{E}-13$ & 0.55 \\
\hline 268 & $8: 59: 00.98$ & $-47: 31: 03.62$ & 99.0 & 97.5 & $1.35 \pm 0.25$ & $2.99 \pm 0.92$ & $1.24 \mathrm{E}-14$ & $2.84 \mathrm{E}-14$ & 0.69 \\
\hline 269 & $8: 59: 00.88$ & $-47: 29: 42.72$ & 30.0 & 27.6 & $2.41 \pm 1.22$ & $10.00 \pm \ldots$ & $5.46 \mathrm{E}-15$ & $1.03 \mathrm{E}-14$ & 0.35 \\
\hline 270 & 8:59:00.91 & $-47: 28: 19.24$ & 45.0 & 43.7 & $1.35 \pm 0.49$ & $10.00 \pm \ldots$ & $8.18 \mathrm{E}-15$ & $1.35 \mathrm{E}-14$ & 0.36 \\
\hline
\end{tabular}


Table 2-Continued

\begin{tabular}{|c|c|c|c|c|c|c|c|c|c|}
\hline $\begin{array}{l}\text { Chandra } \\
\text { ID }\end{array}$ & $\begin{array}{c}\text { RA } \\
(2000)\end{array}$ & $\begin{array}{c}\text { Dec } \\
(2000)\end{array}$ & $\begin{array}{l}\text { Raw } \\
\text { CNT }\end{array}$ & $\begin{array}{l}\text { Net }^{a} \\
\text { CNT }\end{array}$ & $\begin{array}{c}N_{H} \\
10^{22} \mathrm{~cm}^{-2}\end{array}$ & $\begin{array}{l}\mathrm{kT} \\
\mathrm{keV}\end{array}$ & \multicolumn{2}{|c|}{$\left[\mathrm{ergcm}^{-2} \mathrm{~s}^{-1}\right]$} & $\chi^{2}$ \\
\hline 271 & 8:59:00.77 & $-47: 30: 47.11$ & 275.0 & 273.5 & $0.94 \pm 0.11$ & $2.79 \pm 0.49$ & $3.02 \mathrm{E}-14$ & $6.36 \mathrm{E}-14$ & 0.80 \\
\hline 272 & $8: 59: 00.70$ & $-47: 30: 51.09$ & 190.0 & 188.5 & $2.67 \pm 0.28$ & $2.41 \pm 0.33$ & $2.79 \mathrm{E}-14$ & $9.54 \mathrm{E}-14$ & 0.79 \\
\hline 273 & $8: 59: 00.36$ & $-47: 25: 58.37$ & 113.0 & 109.3 & $2.86 \pm 0.41$ & $2.32 \pm 0.42$ & $1.78 \mathrm{E}-14$ & $6.47 \mathrm{E}-14$ & 0.63 \\
\hline 274 & $8: 59: 00.31$ & $-47: 31: 14.55$ & 124.0 & 122.5 & $5.25 \pm 0.77$ & $3.72 \pm 0.81$ & $2.64 \mathrm{E}-14$ & $9.00 \mathrm{E}-14$ & 0.75 \\
\hline 275 & $8: 59: 00.32$ & $-47: 29: 40.51$ & 53.0 & 50.9 & $0.96 \pm 0.30$ & $3.42 \pm 2.32$ & $6.13 \mathrm{E}-15$ & $1.20 \mathrm{E}-14$ & 0.36 \\
\hline 276 & $8: 59: 00.26$ & $-47: 27: 58.77$ & 18.0 & 13.2 & $1.05 \pm 0.64$ & $2.75 \pm 4.83$ & $1.79 \mathrm{E}-15$ & $3.94 \mathrm{E}-15$ & 0.41 \\
\hline 277 & $8: 59: 00.17$ & $-47: 33: 11.71$ & 46.0 & 40.5 & $1.27 \pm 0.37$ & $2.19 \pm 1.15$ & $4.44 \mathrm{E}-15$ & $1.18 \mathrm{E}-14$ & 0.55 \\
\hline 278 & $8: 59: 00.10$ & $-47: 32: 19.49$ & 35.0 & 24.8 & $7.18 \pm 1.84$ & $1.41 \pm 0.25$ & $4.72 \mathrm{E}-15$ & $6.09 \mathrm{E}-14$ & 0.52 \\
\hline 279 & $8: 59: 00.12$ & $-47: 30: 07.09$ & 45.0 & 43.5 & $1.27 \pm 0.37$ & $2.47 \pm 1.12$ & $5.80 \mathrm{E}-15$ & $1.44 \mathrm{E}-14$ & 0.62 \\
\hline 280 & 8:59:00.07 & $-47: 30: 40.76$ & 14.0 & 12.5 & $5.25 \pm 2.25$ & $1.55 \pm 0.61$ & $1.86 \mathrm{E}-15$ & $1.56 \mathrm{E}-14$ & 0.52 \\
\hline 281 & 8:59:00.00 & $-47: 31: 09.46$ & 113.0 & 111.5 & $11.80 \pm 1.87$ & $7.60 \pm 9.45$ & $3.41 \mathrm{E}-14$ & $1.23 \mathrm{E}-13$ & 0.73 \\
\hline 282 & $8: 58: 59.94$ & $-47: 29: 47.51$ & 129.0 & 126.1 & $2.38 \pm 0.32$ & $2.44 \pm 0.45$ & $1.82 \mathrm{E}-14$ & $5.85 \mathrm{E}-14$ & 0.51 \\
\hline 283 & $8: 58: 59.87$ & $-47: 30: 14.54$ & 36.0 & 34.5 & $1.55 \pm 0.53$ & $4.28 \pm 6.18$ & $5.64 \mathrm{E}-15$ & $1.16 \mathrm{E}-14$ & 0.59 \\
\hline 284 & $8: 58: 59.64$ & $-47: 32: 28.86$ & 30.0 & 21.3 & $11.64 \pm 2.65$ & $0.96 \pm 0.12$ & $3.94 \mathrm{E}-15$ & $2.47 \mathrm{E}-13$ & 0.37 \\
\hline 285 & $8: 58: 59.54$ & $-47: 29: 52.38$ & 36.0 & 32.8 & $2.56 \pm 0.78$ & $3.30 \pm 1.91$ & $5.37 \mathrm{E}-15$ & $1.47 \mathrm{E}-14$ & 0.44 \\
\hline 286 & $8: 58: 59.42$ & $-47: 32: 37.71$ & 214.0 & 207.0 & $5.10 \pm 0.66$ & $10.00 \mathrm{v} 23.26$ & $5.21 \mathrm{E}-14$ & $1.24 \mathrm{E}-13$ & 0.56 \\
\hline 287 & $8: 58: 59.44$ & $-47: 31: 11.03$ & 32.0 & 30.5 & $6.82 \pm 1.54$ & $1.50 \pm 0.28$ & $4.86 \mathrm{E}-15$ & $5.30 \mathrm{E}-14$ & 0.59 \\
\hline 288 & $8: 58: 59.17$ & $-47: 32: 32.72$ & 48.0 & 41.4 & $7.95 \pm 2.25$ & $3.01 \pm 0.93$ & $9.81 \mathrm{E}-15$ & $4.81 \mathrm{E}-14$ & 0.33 \\
\hline 289 & 8:58:59.09 & $-47: 32: 16.95$ & 24.0 & 13.5 & $2.18 \pm 1.95$ & $10.00 \pm \ldots$ & $3.11 \mathrm{E}-15$ & $5.74 \mathrm{E}-15$ & 0.55 \\
\hline 290 & $8: 58: 59.18$ & $-47: 26: 58.26$ & 65.0 & 60.1 & $2.28 \pm 0.37$ & $1.4 \pm 0.27$ & $2.02 \mathrm{E}-14$ & $1.04 \mathrm{E}-13$ & 0.52 \\
\hline 291 & $8: 58: 59.11$ & $-47: 30: 34.11$ & 35.0 & 32.2 & $12.44 \pm 3.36$ & $2.93 \pm 0.88$ & $9.06 \mathrm{E}-15$ & $5.96 \mathrm{E}-14$ & 0.42 \\
\hline 292 & $8: 58: 59.12$ & $-47: 28: 09.31$ & 165.0 & 158.9 & $1.10 \pm 0.17$ & $2.79 \pm 0.69$ & $1.95 \mathrm{E}-14$ & $4.32 \mathrm{E}-14$ & 0.63 \\
\hline 293 & $8: 58: 58.91$ & $-47: 33: 44.36$ & 12.0 & 7.2 & $1.44 \pm 0.80$ & $1.14 \pm 0.75$ & 7.33E-16 & $3.82 \mathrm{E}-15$ & 0.47 \\
\hline 294 & $8: 58: 58.89$ & $-47: 30: 54.68$ & 113.0 & 109.6 & $4.46 \pm 0.48$ & $1.44 \pm 0.12$ & $1.60 \mathrm{E}-14$ & $1.34 \mathrm{E}-13$ & 0.49 \\
\hline 295 & $8: 58: 58.88$ & $-47: 27: 25.15$ & 23.0 & 17.6 & $1.02 \pm 0.52$ & $2.22 \pm 1.78$ & $2.17 \mathrm{E}-15$ & $5.26 \mathrm{E}-15$ & 0.31 \\
\hline 296 & $8: 58: 58.72$ & $-47: 31: 04.70$ & 67.0 & 64.2 & $4.54 \pm 0.85$ & $2.23 \pm 0.44$ & $1.12 \mathrm{E}-14$ & $5.37 \mathrm{E}-14$ & 0.34 \\
\hline 297 & $8: 58: 58.59$ & $-47: 31: 10.52$ & 135.0 & 131.8 & $1.41 \pm 0.27$ & $6.59 \pm 9.79$ & $2.13 \mathrm{E}-14$ & $3.80 \mathrm{E}-14$ & 0.43 \\
\hline 298 & $8: 58: 58.64$ & $-47: 30: 16.90$ & 16.0 & 13.3 & $2.05 \pm 1.28$ & $3.84 \pm \ldots$ & $2.07 \mathrm{E}-15$ & $4.83 \mathrm{E}-15$ & 0.31 \\
\hline 299 & $8: 58: 58.52$ & $-47: 30: 59.25$ & 167.0 & 164.3 & $6.60 \pm 0.72$ & $2.45 \pm 0.28$ & $3.39 \mathrm{E}-14$ & $1.82 \mathrm{E}-13$ & 0.47 \\
\hline 300 & $8: 58: 58.29$ & $-47: 32: 58.15$ & 60.0 & 54.5 & $1.92 \pm 0.40$ & $1.97 \pm 0.61$ & $6.58 \mathrm{E}-15$ & $2.27 \mathrm{E}-14$ & 0.44 \\
\hline 301 & $8: 58: 58.31$ & $-47: 27: 27.78$ & 60.0 & 54.6 & $0.81 \pm 0.22$ & $1.54 \pm 0.54$ & $4.54 \mathrm{E}-15$ & $1.25 \mathrm{E}-14$ & 0.38 \\
\hline 302 & $8: 58: 58.16$ & $-47: 31: 07.00$ & 21.0 & 19.7 & $8.71 \pm 5.11$ & $10.00 \pm \ldots$ & $5.52 \mathrm{E}-15$ & $1.61 \mathrm{E}-14$ & 0.14 \\
\hline 303 & $8: 58: 58.10$ & $-47: 31: 45.13$ & 29.0 & 20.6 & $3.63 \pm 1.39$ & $2.26 \pm 0.97$ & $3.75 \mathrm{E}-15$ & $1.57 \mathrm{E}-14$ & 0.68 \\
\hline 304 & $8: 58: 57.95$ & $-47: 31: 22.63$ & 23.0 & 20.7 & $4.05 \pm 1.91$ & $10.00 \pm \ldots$ & $4.98 \mathrm{E}-15$ & $1.09 \mathrm{E}-14$ & 0.32 \\
\hline 305 & $8: 58: 57.93$ & $-47: 30: 31.64$ & 32.0 & 30.4 & $1.21 \pm 0.43$ & $2.72 \pm 1.94$ & $3.73 \mathrm{E}-15$ & $8.65 \mathrm{E}-15$ & 0.31 \\
\hline 306 & $8: 58: 57.89$ & $-47: 32: 43.95$ & 31.0 & 26.2 & $1.69 \pm 0.84$ & $10.00 \pm \ldots$ & $4.85 \mathrm{E}-15$ & $8.42 \mathrm{E}-15$ & 0.20 \\
\hline 307 & $8: 58: 57.93$ & $-47: 30: 17.95$ & 30.0 & 27.2 & $2.15 \pm 0.73$ & $2.47 \pm 1.23$ & $3.93 \mathrm{E}-15$ & $1.20 \mathrm{E}-14$ & 0.36 \\
\hline 308 & $8: 58: 57.74$ & $-47: 29: 19.12$ & 39.0 & 36.6 & $0.95 \pm 0.46$ & $10.00 \pm \ldots$ & $6.20 \mathrm{E}-15$ & $9.56 \mathrm{E}-15$ & 0.43 \\
\hline 309 & $8: 58: 57.51$ & $-47: 27: 43.72$ & 28.0 & 21.4 & $5.07 \pm 2.68$ & $5.46 \pm \cdots$ & $4.88 \mathrm{E}-15$ & $1.36 \mathrm{E}-14$ & 0.42 \\
\hline 310 & $8: 58: 57.21$ & $-47: 31: 06.10$ & 56.0 & 49.7 & $20.30 \pm 2.70$ & $1.14 \pm 0.08$ & $1.28 \mathrm{E}-14$ & $8.90 \mathrm{E}-13$ & 0.59 \\
\hline 311 & $8: 58: 57.11$ & $-47: 32: 38.16$ & 24.0 & 17.9 & $6.99 \pm 1.84$ & $1.11 \pm 0.21$ & $3.06 \mathrm{E}-15$ & $6.70 \mathrm{E}-14$ & 0.31 \\
\hline 312 & $8: 58: 56.84$ & $-47: 33: 06.20$ & 14.0 & 10.7 & $7.48 \pm 1.71$ & $0.56 \pm 0.07$ & $1.42 \mathrm{E}-15$ & $4.14 \mathrm{E}-13$ & 0.28 \\
\hline 313 & $8: 58: 55.81$ & $-47: 32: 51.28$ & 114.0 & 108.0 & $2.87 \pm 0.36$ & $1.83 \pm 0.30$ & $1.57 \mathrm{E}-14$ & $7.11 \mathrm{E}-14$ & 0.59 \\
\hline 314 & $8: 58: 55.58$ & $-47: 27: 44.84$ & 39.0 & 33.1 & $1.91 \pm 1.19$ & $10.00 \pm \ldots$ & $6.11 \mathrm{E}-15$ & $1.09 \mathrm{E}-14$ & 0.57 \\
\hline 315 & $8: 58: 55.48$ & $-47: 30: 24.32$ & 243.0 & 235.2 & $2.91 \pm 0.33$ & $4.82 \pm 1.19$ & $4.55 \mathrm{E}-14$ & $1.09 \mathrm{E}-13$ & 0.83 \\
\hline
\end{tabular}


Table 2-Continued

\begin{tabular}{|c|c|c|c|c|c|c|c|c|c|}
\hline $\begin{array}{l}\text { Chandra } \\
\text { ID }\end{array}$ & $\begin{array}{c}\text { RA } \\
(2000)\end{array}$ & $\begin{array}{c}\text { Dec } \\
(2000)\end{array}$ & $\begin{array}{l}\text { Raw } \\
\text { CNT }\end{array}$ & $\begin{array}{l}\mathrm{Net}^{\mathrm{a}} \\
\mathrm{CNT}\end{array}$ & $\begin{array}{c}N_{H} \\
10^{22} \mathrm{~cm}^{-2}\end{array}$ & $\begin{array}{l}\mathrm{kT} \\
\mathrm{keV}\end{array}$ & $\begin{array}{r}\text { Abs. Flux } \\
{[e r}\end{array}$ & $\begin{array}{l}\text { Tot. UnAbs. Flux } \\
\left.\mathrm{gcm}^{-2} \mathrm{~s}^{-1}\right]\end{array}$ & $\chi^{2}$ \\
\hline 316 & $8: 58: 55.24$ & $-47: 31: 54.78$ & 267.0 & 260.1 & $0.61 \pm 0.04$ & $0.41 \pm 0.02$ & $1.38 \mathrm{E}-14$ & $1.23 \mathrm{E}-13$ & 0.82 \\
\hline 317 & $8: 58: 55.23$ & $-47: 31: 50.07$ & 293.0 & 286.0 & $0.06 \pm 0.05$ & $10.00 \pm 16.90$ & 3.37E-14 & $3.67 \mathrm{E}-14$ & 0.75 \\
\hline 318 & $8: 58: 55.11$ & $-47: 35: 00.41$ & 114.0 & 100.6 & $1.47 \pm 0.26$ & $2.23 \pm 0.56$ & $1.24 \mathrm{E}-14$ & $3.46 \mathrm{E}-14$ & 0.77 \\
\hline 319 & $8: 58: 55.06$ & $-47: 31: 38.33$ & 26.0 & 19.7 & $3.64 \pm 1.98$ & $5.91 \pm \ldots$ & $4.61 \mathrm{E}-15$ & $1.11 \mathrm{E}-14$ & 0.52 \\
\hline 320 & $8: 58: 54.95$ & $-47: 26: 58.66$ & 26.0 & 20.9 & $0.89 \pm 0.59$ & $2.32 \pm 1.85$ & $2.38 \mathrm{E}-15$ & $5.36 \mathrm{E}-15$ & 0.52 \\
\hline 321 & $8: 58: 54.83$ & $-47: 34: 33.47$ & 105.0 & 91.1 & $3.07 \pm 0.39$ & $1.31 \pm 0.17$ & $1.12 \mathrm{E}-14$ & $8.22 \mathrm{E}-14$ & 0.76 \\
\hline 322 & $8: 58: 54.75$ & $-47: 33: 58.33$ & 27.0 & 22.2 & $3.16 \pm 1.54$ & $8.88 \pm \ldots$ & $5.15 \mathrm{E}-15$ & $1.07 \mathrm{E}-14$ & 0.46 \\
\hline 323 & $8: 58: 54.47$ & $-47: 32: 00.66$ & 21.0 & 14.2 & $2.58 \pm 1.18$ & $2.13 \pm 1.44$ & $2.30 \mathrm{E}-15$ & $8.59 \mathrm{E}-15$ & 0.44 \\
\hline 324 & $8: 58: 54.25$ & $-47: 29: 49.74$ & 148.0 & 141.7 & $0.76 \pm 0.16$ & $3.73 \pm 1.49$ & $1.78 \mathrm{E}-14$ & $3.17 \mathrm{E}-14$ & 0.71 \\
\hline 325 & $8: 58: 54.25$ & $-47: 28: 41.88$ & 320.0 & 313.0 & $4.13 \pm 0.44$ & $10.00 \pm 31.67$ & $7.64 \mathrm{E}-14$ & $1.69 \mathrm{E}-13$ & 0.90 \\
\hline 326 & $8: 58: 54.02$ & $-47: 31: 02.09$ & 47.0 & 40.4 & $5.13 \pm 1.17$ & $2.02 \pm 0.51$ & $7.12 \mathrm{E}-15$ & $4.08 \mathrm{E}-14$ & 0.56 \\
\hline 327 & $8: 58: 53.92$ & $-47: 26: 20.78$ & 33.0 & 30.1 & $0.00 \pm 0.07$ & $1.02 \pm 0.30$ & $5.30 \mathrm{E}-15$ & $5.30 \mathrm{E}-15$ & 0.30 \\
\hline 328 & $8: 58: 53.90$ & $-47: 30: 51.44$ & 706.0 & 699.5 & $1.44 \pm 0.10$ & $4.14 \pm 0.50$ & $1.04 \mathrm{E}-13$ & $2.12 \mathrm{E}-13$ & 0.71 \\
\hline 329 & $8: 58: 53.17$ & $-47: 32: 24.44$ & 19.0 & 13.0 & $1.17 \pm 0.99$ & $10.00 \pm \ldots$ & $2.23 \mathrm{E}-15$ & $3.58 \mathrm{E}-15$ & 0.50 \\
\hline 330 & $8: 58: 52.94$ & $-47: 33: 08.98$ & 51.0 & 45.0 & $4.24 \pm 0.71$ & $1.21 \pm 0.19$ & $6.02 \mathrm{E}-15$ & $6.68 \mathrm{E}-14$ & 0.59 \\
\hline 331 & $8: 58: 51.75$ & $-47: 31: 26.98$ & 60.0 & 55.2 & $10.42 \pm 1.83$ & $1.82 \pm 0.27$ & $1.21 \mathrm{E}-14$ & $1.31 \mathrm{E}-13$ & 0.53 \\
\hline 332 & $8: 58: 50.97$ & $-47: 29: 24.07$ & 23.0 & 17.8 & $0.38 \pm 0.18$ & $0.78 \pm 0.21$ & $1.16 \mathrm{E}-15$ & $3.45 \mathrm{E}-15$ & 0.44 \\
\hline 333 & $8: 58: 50.66$ & $-47: 32: 39.13$ & 56.0 & 51.2 & $0.95 \pm 0.26$ & $1.98 \pm 0.87$ & $5.07 \mathrm{E}-15$ & $1.28 \mathrm{E}-14$ & 0.52 \\
\hline 334 & $8: 58: 50.35$ & $-47: 33: 19.56$ & 307.0 & 301.3 & $0.31 \pm 0.03$ & $0.26 \pm 0.01$ & $2.47 \mathrm{E}-14$ & $1.42 \mathrm{E}-13$ & 0.57 \\
\hline 335 & $8: 58: 49.95$ & $-47: 28: 57.79$ & 39.0 & 33.5 & $2.73 \pm 0.68$ & $1.53 \pm 0.42$ & $4.23 \mathrm{E}-15$ & 2.30E-14 & 0.54 \\
\hline 336 & $8: 58: 49.79$ & $-47: 29: 24.92$ & 12.0 & 6.0 & $0.00 \pm 0.09$ & $1.83 \pm 41.07$ & $7.02 \mathrm{E}-16$ & $7.03 \mathrm{E}-16$ & 0.33 \\
\hline 337 & $8: 58: 49.57$ & $-47: 32: 55.46$ & 44.0 & 39.1 & $1.05 \pm 0.47$ & $9.76 \pm 49.20$ & $6.67 \mathrm{E}-15$ & $1.05 \mathrm{E}-14$ & 0.43 \\
\hline 338 & $8: 58: 49.21$ & $-47: 31: 14.99$ & 41.0 & 37.1 & $0.00 \pm 0.05$ & $0.32 \pm 0.05$ & $4.06 \mathrm{E}-15$ & $4.06 \mathrm{E}-15$ & 0.40 \\
\hline 339 & $8: 58: 48.82$ & $-47: 32: 15.77$ & 55.0 & 51.9 & $1.50 \pm 0.39$ & $3.39 \pm 1.74$ & $7.56 \mathrm{E}-15$ & $1.69 \mathrm{E}-14$ & 0.49 \\
\hline 340 & $8: 58: 48.63$ & $-47: 32: 21.96$ & 95.0 & 91.8 & $3.05 \pm 0.46$ & $1.93 \pm 0.34$ & $1.33 \mathrm{E}-14$ & $5.93 \mathrm{E}-14$ & 0.57 \\
\hline 341 & $8: 58: 47.74$ & $-47: 28: 16.53$ & 89.0 & 84.7 & $0.65 \pm 0.22$ & $5.69 \pm 11.71$ & $1.16 \mathrm{E}-14$ & $1.80 \mathrm{E}-14$ & 0.56 \\
\hline 342 & $8: 58: 47.47$ & $-47: 28: 29.73$ & 11.0 & 5.5 & $0.00 \pm 0.09$ & $1.14 \pm 2.31$ & $5.20 \mathrm{E}-16$ & $5.20 \mathrm{E}-16$ & 0.41 \\
\hline 343 & $8: 58: 43.86$ & $-47: 28: 57.20$ & 304.0 & 288.5 & $0.76 \pm 0.11$ & $3.53 \pm 0.79$ & $3.59 \mathrm{E}-14$ & $6.51 \mathrm{E}-14$ & 0.83 \\
\hline 344 & $8: 59: 56.04$ & $-47: 30: 03.88$ & 19.0 & 15.4 & $1.44 \pm 1.03$ & $10.00 \pm \cdots$ & $3.01 \mathrm{E}-15$ & $5.04 \mathrm{E}-15$ & 0.46 \\
\hline 345 & $8: 59: 54.77$ & $-47: 28: 43.18$ & 13.0 & 9.4 & $0.00 \pm 0.06$ & $10.00 \pm \ldots$ & $8.98 \mathrm{E}-16$ & $8.98 \mathrm{E}-16$ & 0.28 \\
\hline 346 & $8: 59: 53.90$ & $-47: 27: 42.54$ & 47.0 & 33.0 & $1.90 \pm 0.34$ & $0.87 \pm 0.17$ & $3.23 \mathrm{E}-15$ & $3.69 \mathrm{E}-14$ & 0.68 \\
\hline 347 & $8: 59: 52.70$ & $-47: 27: 37.31$ & 25.0 & 10.8 & $0.71 \pm 0.35$ & $0.90 \pm 0.41$ & $8.70 \mathrm{E}-16$ & $3.70 \mathrm{E}-15$ & 0.68 \\
\hline 348 & $8: 59: 51.54$ & $-47: 33: 10.03$ & 22.0 & 17.7 & $0.35 \pm 0.27$ & $1.38 \pm 1.38$ & $1.31 \mathrm{E}-15$ & $2.67 \mathrm{E}-15$ & 0.37 \\
\hline 349 & $8: 59: 51.40$ & $-47: 33: 23.72$ & 32.0 & 19.3 & $1.32 \pm 0.55$ & $1.32 \pm 0.79$ & $1.85 \mathrm{E}-15$ & $7.64 \mathrm{E}-15$ & 0.63 \\
\hline 350 & $8: 59: 49.95$ & $-47: 31: 43.14$ & 13.0 & 9.5 & $6.10 \pm 4.36$ & $3.62 \pm 7.36$ & $2.43 \mathrm{E}-15$ & $9.04 \mathrm{E}-15$ & 0.43 \\
\hline 351 & $8: 59: 49.59$ & $-47: 31: 17.07$ & 18.0 & 13.7 & $0.57 \pm 0.65$ & $10.00 \pm \ldots$ & $2.07 \mathrm{E}-15$ & $2.91 \mathrm{E}-15$ & 0.45 \\
\hline 352 & $8: 59: 49.11$ & $-47: 32: 27.59$ & 25.0 & 20.1 & $2.93 \pm 1.20$ & $2.38 \pm 1.39$ & $3.14 \mathrm{E}-15$ & $1.13 \mathrm{E}-14$ & 0.51 \\
\hline 353 & $8: 59: 48.38$ & $-47: 26: 40.06$ & 33.0 & 28.9 & $0.72 \pm 0.33$ & $2.04 \pm 1.54$ & $2.80 \mathrm{E}-15$ & $6.25 \mathrm{E}-15$ & 0.40 \\
\hline 354 & $8: 59: 45.70$ & $-47: 28: 41.82$ & 26.0 & 22.7 & $0.00 \pm 0.10$ & $1.05 \pm 0.45$ & $1.58 \mathrm{E}-15$ & $1.58 \mathrm{E}-15$ & 0.42 \\
\hline 355 & $8: 59: 45.24$ & $-47: 31: 01.59$ & 21.0 & 16.6 & $3.39 \pm 3.53$ & $10.00 \pm \ldots$ & $3.23 \mathrm{E}-15$ & $6.72 \mathrm{E}-15$ & 0.49 \\
\hline 356 & $8: 59: 42.77$ & $-47: 32: 59.45$ & 7.0 & 3.9 & $0.00 \pm 0.15$ & $1.36 \pm 13.85$ & 7.07E-16 & 7.07E-16 & 0.35 \\
\hline 357 & $8: 59: 42.17$ & $-47: 30: 43.00$ & 24.0 & 22.4 & $12.43 \pm 5.86$ & $9.55 \pm \ldots$ & $7.00 \mathrm{E}-15$ & $2.44 \mathrm{E}-14$ & 0.34 \\
\hline 358 & $8: 59: 40.88$ & $-47: 25: 26.68$ & 29.0 & 25.2 & $0.08 \pm 0.18$ & $1.79 \pm 2.65$ & $2.02 \mathrm{E}-15$ & $2.56 \mathrm{E}-15$ & 0.49 \\
\hline 359 & $8: 59: 39.05$ & $-47: 27: 19.37$ & 10.0 & 6.8 & $1.21 \pm 1.52$ & $10.00 \pm \ldots$ & $5.33 \mathrm{E}-15$ & $8.61 \mathrm{E}-15$ & 0.41 \\
\hline 360 & $8: 59: 37.11$ & $-47: 28: 15.41$ & 9.0 & 7.9 & $1.42 \pm 1.39$ & $10.00 \pm \ldots$ & $1.43 \mathrm{E}-15$ & $2.38 \mathrm{E}-15$ & 0.22 \\
\hline
\end{tabular}


Table 2-Continued

\begin{tabular}{|c|c|c|c|c|c|c|c|c|c|}
\hline $\begin{array}{c}\text { Chandra } \\
\text { ID }\end{array}$ & $\begin{array}{c}\text { RA } \\
(2000)\end{array}$ & $\begin{array}{c}\text { Dec } \\
(2000)\end{array}$ & $\begin{array}{l}\text { Raw } \\
\text { CNT }\end{array}$ & $\begin{array}{l}\text { Net }^{a} \\
\text { CNT }\end{array}$ & $\begin{array}{c}N_{H} \\
10^{22} \mathrm{~cm}^{-2}\end{array}$ & $\begin{array}{l}\mathrm{kT} \\
\mathrm{keV}\end{array}$ & \multicolumn{2}{|c|}{$\left[\mathrm{erg} \mathrm{cm}^{-2} \mathrm{~s}^{-1}\right]$} & $\chi^{2}$ \\
\hline 361 & 8:59:36.11 & $-47: 25: 59.30$ & 23.0 & 19.8 & $0.68 \pm 0.44$ & $3.20 \pm \ldots$ & $2.21 \mathrm{E}-15$ & $4.01 \mathrm{E}-15$ & 0.43 \\
\hline 362 & 8:59:34.43 & $-47: 27: 15.55$ & 24.0 & 22.9 & $0.89 \pm 0.32$ & $1.24 \pm 0.71$ & $1.61 \mathrm{E}-15$ & $5.53 \mathrm{E}-15$ & 0.26 \\
\hline 363 & 8:59:33.65 & $-47: 34: 44.93$ & 14.0 & 11.0 & $0.00 \pm 0.08$ & $4.34 \pm \ldots$ & $9.54 \mathrm{E}-16$ & $9.54 \mathrm{E}-16$ & 0.42 \\
\hline 364 & 8:59:33.23 & $-47: 24: 51.96$ & 13.0 & 9.7 & $0.00 \pm 0.10$ & $7.91 \pm \ldots$ & $1.22 \mathrm{E}-15$ & $1.22 \mathrm{E}-15$ & 0.38 \\
\hline 365 & $8: 59: 30.71$ & $-47: 24: 50.31$ & 25.0 & 20.6 & $2.50 \pm 0.84$ & $1.83 \pm 0.88$ & $2.85 \mathrm{E}-15$ & $1.20 \mathrm{E}-14$ & 0.48 \\
\hline 366 & $8: 59: 29.00$ & $-47: 34: 57.52$ & 10.0 & 6.2 & $0.00 \pm 0.06$ & $1.42 \pm 2.76$ & $5.34 \mathrm{E}-16$ & $5.35 \mathrm{E}-16$ & 0.41 \\
\hline 367 & $8: 59: 28.62$ & $-47: 33: 36.02$ & 11.0 & 9.7 & $0.46 \pm 0.80$ & $10.00 \pm \ldots$ & $1.24 \mathrm{E}-15$ & $1.69 \mathrm{E}-15$ & 0.26 \\
\hline 368 & $8: 59: 26.21$ & $-47: 29: 01.53$ & 11.0 & 9.3 & $4.08 \pm 3.18$ & $7.64 \pm \ldots$ & $2.10 \mathrm{E}-15$ & $4.88 \mathrm{E}-15$ & 0.27 \\
\hline 369 & $8: 59: 25.93$ & $-47: 33: 26.57$ & 16.0 & 14.6 & $13.00 \pm 6.93$ & $10.00 \pm \ldots$ & $4.58 \mathrm{E}-15$ & $1.62 \mathrm{E}-14$ & 0.29 \\
\hline 370 & $8: 59: 22.69$ & $-47: 26: 11.40$ & 14.0 & 8.8 & $2.21 \pm 0.61$ & $0.40 \pm 0.09$ & $5.51 \mathrm{E}-16$ & $5.10 \mathrm{E}-14$ & 0.42 \\
\hline 371 & $8: 59: 21.56$ & $-47: 26: 44.60$ & 12.0 & 10.3 & $0.06 \pm 0.07$ & $0.09 \pm 0.01$ & $3.56 \mathrm{E}-15$ & $1.06 \mathrm{E}-14$ & 0.28 \\
\hline 372 & $8: 59: 21.07$ & $-47: 35: 56.12$ & 17.0 & 13.2 & $0.00 \pm 0.08$ & $3.83 \pm \ldots$ & $1.25 \mathrm{E}-15$ & $1.25 \mathrm{E}-15$ & 0.39 \\
\hline 373 & $8: 59: 20.88$ & $-47: 24: 46.17$ & 74.0 & 69.7 & $3.41 \pm 0.88$ & $10.00 \pm \ldots$ & $1.63 \mathrm{E}-14$ & $3.39 \mathrm{E}-14$ & 0.59 \\
\hline 374 & $8: 59: 19.77$ & $-47: 24: 02.10$ & 28.0 & 17.9 & $2.29 \pm 2.07$ & $10.00 \pm \cdots$ & $5.12 \mathrm{E}-15$ & $9.56 \mathrm{E}-15$ & 0.69 \\
\hline 375 & $8: 59: 18.14$ & $-47: 35: 24.31$ & 10.0 & 6.8 & $0.00 \pm 0.36$ & $10.00 \pm \ldots$ & $6.69 \mathrm{E}-16$ & $6.69 \mathrm{E}-16$ & 0.39 \\
\hline 376 & $8: 59: 18.10$ & $-47: 24: 43.15$ & 23.0 & 19.7 & $16.73 \pm 5.42$ & $2.93 \pm 1.09$ & $7.06 \mathrm{E}-15$ & $5.69 \mathrm{E}-14$ & 0.40 \\
\hline 377 & 8:59:17.35 & $-47: 26: 50.90$ & 8.0 & 6.5 & $0.00 \pm 0.18$ & $1.53 \pm 28.69$ & $5.39 \mathrm{E}-16$ & $5.39 \mathrm{E}-16$ & 0.23 \\
\hline 378 & 8:59:16.01 & $-47: 25: 02.38$ & 31.0 & 27.9 & $4.09 \pm 1.63$ & $10.00 \pm \ldots$ & $7.48 \mathrm{E}-15$ & $1.65 \mathrm{E}-14$ & 0.44 \\
\hline 379 & $8: 59: 15.30$ & $-47: 27: 05.95$ & 13.0 & 11.4 & $3.01 \pm 3.90$ & $10.00 \pm \ldots$ & $2.31 \mathrm{E}-15$ & $4.64 \mathrm{E}-15$ & 0.25 \\
\hline 380 & $8: 59: 14.86$ & $-47: 32: 52.60$ & 11.0 & 9.8 & $11.37 \pm 5.32$ & $2.32 \pm 1.10$ & $2.38 \mathrm{E}-15$ & $1.93 \mathrm{E}-14$ & 0.23 \\
\hline 381 & 8:59:14.36 & $-47: 24: 21.56$ & 26.0 & 23.2 & $0.23 \pm 0.24$ & $3.05 \pm 9.02$ & $2.20 \mathrm{E}-15$ & $3.14 \mathrm{E}-15$ & 0.42 \\
\hline 382 & $8: 59: 14.31$ & $-47: 33: 44.94$ & 17.0 & 16.0 & $5.89 \pm 3.16$ & $10.00 \pm \ldots$ & $4.42 \mathrm{E}-15$ & $1.10 \mathrm{E}-14$ & 0.25 \\
\hline 383 & $8: 59: 14.10$ & $-47: 36: 11.59$ & 26.0 & 13.8 & $5.07 \pm 10.33$ & $5.96 \pm \ldots$ & $2.84 \mathrm{E}-15$ & $7.63 \mathrm{E}-15$ & 0.72 \\
\hline 384 & $8: 59: 13.56$ & $-47: 30: 16.27$ & 10.0 & 8.4 & $2.91 \pm 3.72$ & $10.00 \pm \ldots$ & $1.68 \mathrm{E}-15$ & $3.34 \mathrm{E}-15$ & 0.28 \\
\hline 385 & $8: 59: 12.61$ & $-47: 34: 13.85$ & 21.0 & 16.6 & $0.00 \pm 0.07$ & $1.83 \pm 9.55$ & $1.45 \mathrm{E}-15$ & $1.45 \mathrm{E}-15$ & 0.47 \\
\hline 386 & 8:59:10.07 & $-47: 35: 17.80$ & 13.0 & 9.3 & $0.00 \pm 0.20$ & $2.81 \pm \ldots$ & $9.09 \mathrm{E}-16$ & $9.09 \mathrm{E}-16$ & 0.37 \\
\hline 387 & 8:59:09.87 & $-47: 28: 56.78$ & 13.0 & 10.6 & $0.00 \pm 0.25$ & $10.00 \pm \ldots$ & $1.07 \mathrm{E}-15$ & $1.07 \mathrm{E}-15$ & 0.34 \\
\hline 388 & 8:59:09.66 & $-47: 24: 38.88$ & 37.0 & 33.1 & $2.23 \pm 0.61$ & $2.00 \pm 0.87$ & $4.43 \mathrm{E}-15$ & $1.62 \mathrm{E}-14$ & 0.49 \\
\hline 389 & 8:59:09.64 & $-47: 28: 12.12$ & 10.0 & 8.8 & $0.36 \pm 0.48$ & $3.18 \pm \cdots$ & $1.48 \mathrm{E}-15$ & $2.30 \mathrm{E}-15$ & 0.22 \\
\hline 390 & 8:59:09.14 & $-47: 28: 01.06$ & 9.0 & 7.7 & $1.11 \pm 0.60$ & $0.82 \pm 2.39$ & $4.93 \mathrm{E}-16$ & $3.86 \mathrm{E}-15$ & 0.21 \\
\hline 391 & 8:59:08.33 & $-47: 24: 48.40$ & 12.0 & 7.7 & $0.00 \pm 0.15$ & $1.14 \pm 17.40$ & $4.03 \mathrm{E}-16$ & $4.03 \mathrm{E}-16$ & 0.41 \\
\hline 392 & 8:59:08.11 & $-47: 24: 06.74$ & 55.0 & 41.3 & $0.87 \pm 0.11$ & $0.23 \pm 0.02$ & $2.44 \mathrm{E}-15$ & $1.23 \mathrm{E}-13$ & 0.61 \\
\hline 393 & 8:59:07.03 & $-47: 31: 50.51$ & 10.0 & 8.0 & $6.77 \pm 3.59$ & $1.83 \pm 0.91$ & $1.59 \mathrm{E}-15$ & $1.24 \mathrm{E}-14$ & 0.26 \\
\hline 394 & 8:59:06.14 & $-47: 34: 33.86$ & 16.0 & 13.5 & $8.54 \pm 6.46$ & $10.00 \pm \ldots$ & $3.39 \mathrm{E}-15$ & $9.82 \mathrm{E}-15$ & 0.36 \\
\hline 395 & 8:59:05.39 & $-47: 32: 03.74$ & 12.0 & 10.6 & $2.88 \pm 1.39$ & $1.55 \pm 1.01$ & $1.18 \mathrm{E}-15$ & $6.57 \mathrm{E}-15$ & 0.26 \\
\hline 396 & 8:59:03.86 & $-47: 24: 56.99$ & 69.0 & 64.7 & $2.75 \pm 0.54$ & $2.72 \pm 0.82$ & $1.07 \mathrm{E}-14$ & $3.38 \mathrm{E}-14$ & 0.57 \\
\hline 397 & 8:59:03.44 & $-47: 24: 28.19$ & 59.0 & 45.2 & $1.50 \pm 0.45$ & $2.93 \pm 1.68$ & $6.80 \mathrm{E}-15$ & $1.63 \mathrm{E}-14$ & 0.78 \\
\hline 398 & 8:59:02.18 & $-47: 26: 39.85$ & 28.0 & 23.0 & $5.20 \pm 1.30$ & $1.19 \pm 0.24$ & $3.38 \mathrm{E}-15$ & $4.65 \mathrm{E}-14$ & 0.51 \\
\hline 399 & 8:59:01.49 & $-47: 28: 31.44$ & 13.0 & 10.9 & $0.44 \pm 0.44$ & $1.82 \pm 3.10$ & $9.36 \mathrm{E}-16$ & $1.86 \mathrm{E}-15$ & 0.30 \\
\hline 400 & 8:59:00.95 & $-47: 35: 48.38$ & 16.0 & 5.9 & $0.00 \pm 0.35$ & $2.32 \pm 61.46$ & $8.95 \mathrm{E}-16$ & $8.95 \mathrm{E}-16$ & 0.63 \\
\hline 401 & $8: 59: 00.67$ & $-47: 27: 24.61$ & 24.0 & 21.5 & $0.37 \pm 0.34$ & $7.11 \pm \cdots$ & $3.05 \mathrm{E}-15$ & $4.16 \mathrm{E}-15$ & 0.33 \\
\hline 402 & $8: 58: 59.77$ & $-47: 32: 22.24$ & 56.0 & 44.9 & $3.56 \pm 0.91$ & $2.33 \pm 0.72$ & $7.69 \mathrm{E}-15$ & $3.10 \mathrm{E}-14$ & 0.55 \\
\hline 403 & $8: 58: 59.52$ & $-47: 27: 38.06$ & 20.0 & 15.3 & $1.91 \pm 0.67$ & $1.15 \pm 0.56$ & $1.42 \mathrm{E}-15$ & $8.99 \mathrm{E}-15$ & 0.30 \\
\hline 404 & $8: 58: 58.78$ & $-47: 31: 44.72$ & 16.0 & 13.9 & $1.45 \pm 1.36$ & $10.00 \pm \ldots$ & $2.40 \mathrm{E}-15$ & $4.03 \mathrm{E}-15$ & 0.26 \\
\hline 405 & 8:58:58.02 & $-47: 26: 56.34$ & 10.0 & 5.0 & $5.96 \pm 2.81$ & $0.76 \pm 0.25$ & $1.35 \mathrm{E}-15$ & $8.57 \mathrm{E}-14$ & 0.42 \\
\hline
\end{tabular}


Table 2-Continued

\begin{tabular}{|c|c|c|c|c|c|c|c|c|c|}
\hline $\begin{array}{l}\text { Chandra } \\
\text { ID }\end{array}$ & $\begin{array}{c}\text { RA } \\
(2000)\end{array}$ & $\begin{array}{c}\text { Dec } \\
(2000)\end{array}$ & $\begin{array}{l}\text { Raw } \\
\text { CNT }\end{array}$ & $\begin{array}{l}\mathrm{Net}^{\mathrm{a}} \\
\mathrm{CNT}\end{array}$ & $\begin{array}{c}N_{H} \\
10^{22} \mathrm{~cm}^{-2}\end{array}$ & $\begin{array}{l}\mathrm{kT} \\
\mathrm{keV}\end{array}$ & $\begin{array}{l}\text { Abs. Flux } \\
{[e r}\end{array}$ & $\begin{array}{l}\text { Tot. UnAbs. Flux } \\
\left.\mathrm{gcm}^{-2} \mathrm{~s}^{-1}\right]\end{array}$ & $\chi^{2}$ \\
\hline 406 & 8:58:58.00 & $-47: 27: 33.38$ & 26.0 & 19.5 & $0.70 \pm 0.43$ & $2.32 \pm 2.02$ & $2.23 \mathrm{E}-15$ & $4.62 \mathrm{E}-15$ & 0.32 \\
\hline 407 & $8: 58: 57.45$ & $-47: 28: 41.59$ & 21.0 & 14.3 & $1.85 \pm 0.44$ & $0.56 \pm 0.12$ & $1.15 \mathrm{E}-15$ & $3.89 \mathrm{E}-14$ & 0.54 \\
\hline 408 & 8:58:57.36 & $-47: 27: 29.87$ & 17.0 & 10.4 & $1.87 \pm 0.98$ & $1.31 \pm 0.72$ & $1.30 \mathrm{E}-15$ & $6.82 \mathrm{E}-15$ & 0.38 \\
\hline 409 & 8:58:57.06 & $-47: 32: 29.05$ & 28.0 & 21.6 & $3.23 \pm 0.61$ & $0.70 \pm 0.12$ & $2.31 \mathrm{E}-15$ & $9.18 \mathrm{E}-14$ & 0.42 \\
\hline 410 & 8:58:53.69 & $-47: 30: 14.30$ & 18.0 & 13.2 & $6.34 \pm 5.02$ & $2.93 \pm 2.18$ & $2.94 \mathrm{E}-15$ & $1.31 \mathrm{E}-14$ & 0.38 \\
\hline 411 & $8: 58: 53.46$ & $-47: 30: 33.43$ & 16.0 & 10.5 & $0.09 \pm 0.89$ & $10.00 \pm \cdots$ & $1.16 \mathrm{E}-15$ & $1.31 \mathrm{E}-15$ & 0.33 \\
\hline 412 & $8: 58: 52.83$ & $-47: 31: 06.66$ & 18.0 & 12.3 & $5.42 \pm 3.53$ & $2.93 \pm 2.36$ & $2.75 \mathrm{E}-15$ & $1.12 \mathrm{E}-14$ & 0.31 \\
\hline 413 & $8: 58: 52.74$ & $-47: 33: 15.27$ & 36.0 & 30.3 & $3.99 \pm 0.82$ & $0.92 \pm 0.15$ & $3.49 \mathrm{E}-15$ & $7.39 \mathrm{E}-14$ & 0.54 \\
\hline 414 & $8: 58: 52.08$ & $-47: 34: 20.54$ & 29.0 & 12.6 & $6.79 \pm 3.33$ & $1.86 \pm 0.74$ & $3.58 \mathrm{E}-15$ & $2.73 \mathrm{E}-14$ & 0.62 \\
\hline 415 & 8:58:51.61 & $-47: 31: 08.34$ & 26.0 & 21.3 & $1.65 \pm 0.99$ & $8.11 \pm \ldots$ & $4.06 \mathrm{E}-15$ & $7.23 \mathrm{E}-15$ & 0.36 \\
\hline 416 & 8:58:51.38 & $-47: 27: 22.76$ & 25.0 & 19.9 & $0.55 \pm 0.54$ & $10.00 \pm \ldots$ & $2.80 \mathrm{E}-15$ & $3.92 \mathrm{E}-15$ & 0.54 \\
\hline 417 & $8: 58: 50.75$ & $-47: 30: 43.50$ & 19.0 & 13.6 & $17.86 \pm 5.67$ & $0.90 \pm 0.13$ & $2.40 \mathrm{E}-15$ & $3.65 \mathrm{E}-13$ & 0.48 \\
\hline 418 & $8: 58: 50.81$ & $-47: 30: 13.15$ & 21.0 & 16.5 & $1.16 \pm 0.60$ & $1.83 \pm 1.07$ & $2.00 \mathrm{E}-15$ & $5.77 \mathrm{E}-15$ & 0.42 \\
\hline 419 & $8: 58: 50.08$ & $-47: 30: 07.62$ & 34.0 & 30.0 & $1.27 \pm 0.63$ & $10.00 \pm \ldots$ & $5.21 \mathrm{E}-15$ & $8.50 \mathrm{E}-15$ & 0.48 \\
\hline 420 & 8:58:49.10 & $-47: 29: 17.36$ & 38.0 & 32.9 & $0.59 \pm 0.31$ & $4.14 \pm 37.81$ & $3.97 \mathrm{E}-15$ & $6.48 \mathrm{E}-15$ & 0.40 \\
\hline 421 & $8: 58: 48.90$ & $-47: 31: 31.35$ & 16.0 & 11.3 & $2.90 \pm 2.47$ & $10.00 \pm \ldots$ & $2.86 \mathrm{E}-15$ & $5.69 \mathrm{E}-15$ & 0.40 \\
\hline 422 & $8: 58: 47.68$ & $-47: 29: 39.82$ & 19.0 & 12.4 & $0.68 \pm 0.53$ & $2.03 \pm 5.14$ & $1.16 \mathrm{E}-15$ & $2.55 \mathrm{E}-15$ & 0.51 \\
\hline 423 & $8: 58: 47.50$ & $-47: 33: 23.63$ & 85.0 & 70.3 & $13.89 \pm 2.91$ & $5.75 \pm 3.40$ & $2.20 \mathrm{E}-14$ & $9.61 \mathrm{E}-14$ & 0.77 \\
\hline 424 & $8: 58: 46.93$ & $-47: 33: 14.69$ & 44.0 & 30.0 & $0.65 \pm 0.50$ & $10.00 \pm \ldots$ & $4.55 \mathrm{E}-15$ & $6.55 \mathrm{E}-15$ & 0.65 \\
\hline 425 & 8:58:46.70 & $-47: 30: 34.80$ & 24.0 & 19.2 & $0.32 \pm 0.68$ & $10.00 \pm \ldots$ & $2.47 \mathrm{E}-15$ & $3.19 \mathrm{E}-15$ & 0.51 \\
\hline 426 & 8:58:45.61 & $-47: 29: 33.47$ & 56.0 & 49.9 & $0.62 \pm 0.31$ & $10.00 \pm \ldots$ & $7.25 \mathrm{E}-15$ & $1.04 \mathrm{E}-14$ & 0.56 \\
\hline 427 & 8:58:43.58 & $-47: 29: 34.38$ & 50.0 & 34.3 & $0.34 \pm 0.37$ & $10.00 \pm \cdots$ & $4.62 \mathrm{E}-15$ & $6.04 \mathrm{E}-15$ & 0.60 \\
\hline 428 & $8: 59: 57.96$ & $-47: 28: 47.37$ & 24.0 & 12.7 & $1.22 \pm 0.92$ & $3.66 \pm \cdots$ & $1.93 \mathrm{E}-15$ & $3.92 \mathrm{E}-15$ & 0.65 \\
\hline 429 & $8: 59: 48.27$ & $-47: 26: 21.64$ & 20.0 & 10.4 & $0.32 \pm 0.46$ & $2.04 \pm 6.07$ & $1.17 \mathrm{E}-15$ & $2.00 \mathrm{E}-15$ & 0.62 \\
\hline 430 & $8: 59: 27.70$ & $-47: 35: 50.74$ & 19.0 & 15.1 & $2.80 \pm 1.13$ & $1.59 \pm 0.78$ & $1.87 \mathrm{E}-15$ & $9.85 \mathrm{E}-15$ & 0.38 \\
\hline 431 & $8: 59: 26.25$ & $-47: 35: 42.18$ & 15.0 & 11.6 & $8.65 \pm 6.13$ & $3.71 \pm 3.91$ & $3.18 \mathrm{E}-15$ & $1.38 \mathrm{E}-14$ & 0.38 \\
\hline 432 & $8: 59: 25.55$ & $-47: 29: 11.40$ & 13.0 & 11.4 & $0.57 \pm 0.63$ & $4.97 \pm \ldots$ & $1.26 \mathrm{E}-15$ & $1.95 \mathrm{E}-15$ & 0.28 \\
\hline 433 & $8: 59: 24.89$ & $-47: 24: 51.24$ & 14.0 & 9.7 & $0.25 \pm 0.22$ & $0.71 \pm 0.29$ & $6.27 \mathrm{E}-16$ & $1.42 \mathrm{E}-15$ & 0.45 \\
\hline 434 & $8: 59: 21.92$ & $-47: 34: 33.37$ & 14.0 & 10.5 & $3.49 \pm 5.05$ & $10.00 \pm \ldots$ & $2.39 \mathrm{E}-15$ & $5.01 \mathrm{E}-15$ & 0.42 \\
\hline 435 & 8:59:11.75 & $-47: 31: 32.47$ & 11.0 & 8.7 & $1.13 \pm 1.65$ & $10.00 \pm \ldots$ & $1.43 \mathrm{E}-15$ & $2.27 \mathrm{E}-15$ & 0.31 \\
\hline 436 & 8:59:09.44 & $-47: 34: 57.09$ & 15.0 & 11.1 & $0.97 \pm 0.66$ & $2.01 \pm 3.31$ & $1.05 \mathrm{E}-15$ & $2.66 \mathrm{E}-15$ & 0.41 \\
\hline 437 & 8:59:06.66 & $-47: 36: 17.20$ & 27.0 & 15.1 & $0.00 \pm 0.09$ & $2.32 \pm 28.79$ & $1.58 \mathrm{E}-15$ & $1.58 \mathrm{E}-15$ & 0.68 \\
\hline 438 & 8:58:54.40 & $-47: 25: 51.54$ & 31.0 & 17.2 & $0.82 \pm 0.52$ & $1.83 \pm 2.38$ & $1.76 \mathrm{E}-15$ & $4.36 \mathrm{E}-15$ & 0.65 \\
\hline 439 & 8:58:53.37 & $-47: 26: 14.75$ & 35.0 & 22.2 & $0.73 \pm 0.42$ & $2.77 \pm 2.99$ & $5.91 \mathrm{E}-15$ & $1.16 \mathrm{E}-14$ & 0.65 \\
\hline 440 & 8:58:47.15 & $-47: 31: 53.49$ & 21.0 & 16.6 & $4.75 \pm 1.29$ & $0.92 \pm 0.19$ & $2.10 \mathrm{E}-15$ & $5.39 \mathrm{E}-14$ & 0.46 \\
\hline 441 & $8: 58: 42.76$ & $-47: 30: 15.39$ & 39.0 & 23.4 & $1.20 \pm 0.15$ & $0.15 \pm 0.01$ & $1.39 \mathrm{E}-15$ & $8.48 \mathrm{E}-13$ & 0.67 \\
\hline 442 & 8:59:08.61 & $-47: 25: 42.89$ & 11.0 & 7.9 & $1.08 \pm 1.51$ & $10.00 \pm \cdots$ & $1.38 \mathrm{E}-15$ & $2.18 \mathrm{E}-15$ & 0.35 \\
\hline 443 & 9:00:09.36 & $-47: 28: 33.71$ & 86.0 & 59.1 & $2.89 \pm 1.06$ & $10.00 \pm \ldots$ & $1.47 \mathrm{E}-14$ & $2.92 \mathrm{E}-14$ & 1.06 \\
\hline 444 & 9:00:06.80 & $-47: 30: 32.34$ & 44.0 & 32.3 & $0.49 \pm 0.28$ & $2.32 \pm 2.28$ & $3.14 \mathrm{E}-15$ & $5.84 \mathrm{E}-15$ & 0.70 \\
\hline 445 & 9:00:06.53 & $-47: 31: 48.75$ & 24.0 & 13.4 & $0.00 \pm 0.04$ & $1.44 \pm 2.41$ & $1.44 \mathrm{E}-15$ & $1.44 \mathrm{E}-15$ & 0.68 \\
\hline 446 & 9:00:04.82 & $-47: 27: 07.95$ & 120.0 & 94.8 & $0.42 \pm 0.15$ & $3.33 \pm 1.88$ & $1.16 \mathrm{E}-14$ & $1.85 \mathrm{E}-14$ & 0.96 \\
\hline 447 & 9:00:04.41 & $-47: 29: 46.56$ & 78.0 & 66.0 & $0.00 \pm 0.05$ & $0.31 \pm 0.03$ & $7.03 \mathrm{E}-15$ & $7.03 \mathrm{E}-15$ & 0.76 \\
\hline 448 & $8: 59: 59.93$ & $-47: 34: 09.20$ & 58.0 & 44.8 & $4.55 \pm 1.76$ & $10.00 \pm \cdots$ & $1.15 \mathrm{E}-14$ & $2.61 \mathrm{E}-14$ & 0.80 \\
\hline 449 & $8: 59: 58.23$ & $-47: 27: 31.28$ & 27.0 & 15.8 & $0.00 \pm 0.16$ & $5.35 \pm \cdots$ & $2.09 \mathrm{E}-15$ & $2.09 \mathrm{E}-15$ & 0.59 \\
\hline 450 & $8: 59: 57.95$ & $-47: 27: 08.49$ & 176.0 & 163.4 & $0.00 \pm 0.05$ & $1.05 \pm 0.06$ & $1.29 \mathrm{E}-14$ & $1.30 \mathrm{E}-14$ & 0.74 \\
\hline
\end{tabular}


Table 2-Continued

\begin{tabular}{|c|c|c|c|c|c|c|c|c|c|}
\hline $\begin{array}{l}\text { Chandra } \\
\text { ID }\end{array}$ & $\begin{array}{c}\mathrm{RA} \\
(2000)\end{array}$ & $\begin{array}{c}\text { Dec } \\
(2000)\end{array}$ & $\begin{array}{l}\text { Raw } \\
\text { CNT }\end{array}$ & $\begin{array}{l}\text { Net }^{\mathrm{a}} \\
\mathrm{CNT}\end{array}$ & $\begin{array}{c}N_{H} \\
10^{22} \mathrm{~cm}^{-2}\end{array}$ & $\begin{array}{l}\mathrm{kT} \\
\mathrm{keV}\end{array}$ & $\begin{array}{l}\text { Abs. Flux } \\
\qquad e r\end{array}$ & $\begin{array}{l}\text { Tot. UnAbs. Flux } \\
\left.\mathrm{cm}^{-2} \mathrm{~s}^{-1}\right]\end{array}$ & $\chi^{2}$ \\
\hline 451 & $8: 59: 57.93$ & $-47: 27: 02.19$ & 201.0 & 188.2 & $0.00 \pm 0.04$ & $1.01 \pm 0.06$ & $1.47 \mathrm{E}-14$ & $1.47 \mathrm{E}-14$ & 0.78 \\
\hline 452 & $8: 59: 57.40$ & $-47: 27: 28.62$ & 25.0 & 13.3 & $0.00 \pm 0.06$ & $1.93 \pm 6.89$ & $1.24 \mathrm{E}-15$ & $1.24 \mathrm{E}-15$ & 0.58 \\
\hline 453 & $8: 59: 56.07$ & $-47: 33: 04.63$ & 399.0 & 385.2 & $1.08 \pm 0.04$ & $0.30 \pm 0.01$ & $2.37 \mathrm{E}-14$ & $1.07 \mathrm{E}-12$ & 0.74 \\
\hline 454 & $8: 59: 55.19$ & $-47: 37: 19.36$ & 160.0 & 143.1 & $0.23 \pm 0.08$ & $1.27 \pm 0.16$ & $2.15 \mathrm{E}-14$ & $3.85 \mathrm{E}-14$ & 0.87 \\
\hline 455 & $8: 59: 41.93$ & $-47: 24: 19.79$ & 27.0 & 16.8 & $0.00 \pm 0.32$ & $4.81 \pm \ldots$ & $1.97 \mathrm{E}-15$ & $1.97 \mathrm{E}-15$ & 0.74 \\
\hline 456 & $8: 59: 28.98$ & $-47: 20: 56.90$ & 131.0 & 111.2 & $0.48 \pm 0.13$ & $2.05 \pm 0.71$ & $1.16 \mathrm{E}-14$ & $2.26 \mathrm{E}-14$ & 0.96 \\
\hline 457 & $8: 59: 28.70$ & $-47: 38: 19.69$ & 43.0 & 16.5 & $0.00 \pm 0.29$ & $4.50 \pm \ldots$ & $1.23 \mathrm{E}-15$ & $1.23 \mathrm{E}-15$ & 0.75 \\
\hline 458 & $8: 59: 25.12$ & $-47: 23: 26.22$ & 21.0 & 10.3 & $0.00 \pm 0.06$ & $1.83 \pm 9.86$ & $9.92 \mathrm{E}-16$ & $9.92 \mathrm{E}-16$ & 0.69 \\
\hline 459 & $8: 59: 24.68$ & $-47: 38: 29.13$ & 144.0 & 121.0 & $0.62 \pm 0.21$ & $10.00 \pm 23.76$ & $1.85 \mathrm{E}-14$ & $2.65 \mathrm{E}-14$ & 0.95 \\
\hline 460 & $8: 59: 24.76$ & $-47: 38: 02.54$ & 51.0 & 40.6 & $1.46 \pm 0.23$ & $0.69 \pm 0.13$ & $2.75 \mathrm{E}-15$ & $4.26 \mathrm{E}-14$ & 0.50 \\
\hline 461 & $8: 59: 23.62$ & $-47: 37: 50.65$ & 82.0 & 71.8 & $1.42 \pm 0.41$ & $5.74 \pm 32.56$ & $1.23 \mathrm{E}-14$ & $2.27 \mathrm{E}-14$ & 0.69 \\
\hline 462 & $8: 59: 15.62$ & $-47: 37: 23.68$ & 40.0 & 28.4 & $2.60 \pm 1.17$ & $10.00 \pm \ldots$ & $6.63 \mathrm{E}-15$ & $1.28 \mathrm{E}-14$ & 0.77 \\
\hline 463 & $8: 59: 14.45$ & $-47: 22: 40.93$ & 22.0 & 9.7 & $0.00 \pm 0.27$ & $10.00 \pm \cdots$ & $1.54 \mathrm{E}-15$ & $1.54 \mathrm{E}-15$ & 0.66 \\
\hline 464 & $8: 59: 10.54$ & $-47: 23: 49.86$ & 37.0 & 25.2 & $0.23 \pm 0.30$ & $3.75 \pm 32.05$ & $2.98 \mathrm{E}-15$ & $4.11 \mathrm{E}-15$ & 0.59 \\
\hline 465 & $8: 59: 08.83$ & $-47: 36: 37.95$ & 51.0 & 39.9 & $0.00 \pm 0.07$ & $0.47 \pm 0.12$ & $3.12 \mathrm{E}-15$ & $3.12 \mathrm{E}-15$ & 0.63 \\
\hline 466 & $8: 59: 08.10$ & $-47: 23: 45.69$ & 15.0 & 0.9 & $17.90 \pm \cdots$ & $1.42 \pm 1.99$ & $4.40 \mathrm{E}-16$ & $1.34 \mathrm{E}-14$ & 0.51 \\
\hline 467 & $8: 59: 07.27$ & $-47: 23: 36.02$ & 52.0 & 38.7 & $0.74 \pm 0.43$ & $10.00 \pm \ldots$ & $6.48 \mathrm{E}-15$ & $9.55 \mathrm{E}-15$ & 0.68 \\
\hline 468 & $8: 59: 04.22$ & $-47: 23: 21.55$ & 133.0 & 120.0 & $2.59 \pm 0.46$ & $4.23 \pm 1.54$ & $2.29 \mathrm{E}-14$ & $5.57 \mathrm{E}-14$ & 0.82 \\
\hline 469 & $8: 59: 01.47$ & $-47: 23: 24.83$ & 84.0 & 71.9 & $1.73 \pm 0.38$ & $2.61 \pm 0.95$ & $1.04 \mathrm{E}-14$ & $2.82 \mathrm{E}-14$ & 0.63 \\
\hline 470 & $8: 59: 00.24$ & $-47: 23: 52.79$ & 19.0 & 5.4 & $0.00 \pm 0.05$ & $0.05 \pm 0.01$ & $3.96 \mathrm{E}-14$ & $3.97 \mathrm{E}-14$ & 0.51 \\
\hline 471 & $8: 58: 59.85$ & $-47: 35: 51.54$ & 40.0 & 30.5 & $2.39 \pm 0.69$ & $1.44 \pm 0.36$ & $3.68 \mathrm{E}-15$ & $1.98 \mathrm{E}-14$ & 0.69 \\
\hline 472 & $8: 58: 57.52$ & $-47: 24: 26.28$ & 50.0 & 36.8 & $0.47 \pm 0.21$ & $1.36 \pm 0.82$ & $2.97 \mathrm{E}-15$ & $6.92 \mathrm{E}-15$ & 0.56 \\
\hline 473 & $8: 58: 56.09$ & $-47: 35: 20.99$ & 42.0 & 31.4 & $0.89 \pm 0.54$ & $9.99 \pm \ldots$ & $4.56 \mathrm{E}-15$ & $6.93 \mathrm{E}-15$ & 0.70 \\
\hline 474 & $8: 58: 55.06$ & $-47: 25: 02.52$ & 48.0 & 32.7 & $0.10 \pm 0.12$ & $0.68 \pm 0.18$ & $2.16 \mathrm{E}-15$ & $3.17 \mathrm{E}-15$ & 0.64 \\
\hline 475 & $8: 58: 54.50$ & $-47: 24: 29.94$ & 131.0 & 115.3 & $0.72 \pm 0.16$ & $2.41 \pm 0.79$ & $1.31 \mathrm{E}-14$ & $2.69 \mathrm{E}-14$ & 0.64 \\
\hline 476 & $8: 58: 52.92$ & $-47: 23: 53.60$ & 18.0 & 9.0 & $0.15 \pm 1.38$ & $10.00 \pm \ldots$ & $1.15 \mathrm{E}-15$ & $1.36 \mathrm{E}-15$ & 0.62 \\
\hline 477 & $8: 58: 52.22$ & $-47: 24: 56.11$ & 39.0 & 26.0 & $0.84 \pm 0.44$ & $2.94 \pm 3.87$ & $3.64 \mathrm{E}-15$ & $7.22 \mathrm{E}-15$ & 0.63 \\
\hline 478 & $8: 58: 51.10$ & $-47: 34: 37.71$ & 40.0 & 27.1 & $2.01 \pm 0.73$ & $2.73 \pm 1.80$ & $4.18 \mathrm{E}-15$ & $1.16 \mathrm{E}-14$ & 0.57 \\
\hline 479 & $8: 58: 49.12$ & $-47: 34: 09.82$ & 74.0 & 60.6 & $1.21 \pm 0.32$ & $3.02 \pm 1.64$ & $8.05 \mathrm{E}-15$ & $1.77 \mathrm{E}-14$ & 0.75 \\
\hline 480 & $8: 58: 44.37$ & $-47: 32: 53.85$ & 118.0 & 104.2 & $0.80 \pm 0.17$ & $2.02 \pm 0.60$ & $1.05 \mathrm{E}-14$ & $2.46 \mathrm{E}-14$ & 0.82 \\
\hline 481 & $8: 58: 44.14$ & $-47: 34: 40.51$ & 47.0 & 35.9 & $6.20 \pm 1.80$ & $2.22 \pm 0.62$ & $7.74 \mathrm{E}-15$ & $4.47 \mathrm{E}-14$ & 0.66 \\
\hline 482 & $8: 58: 42.61$ & $-47: 31: 55.93$ & 57.0 & 43.7 & $2.69 \pm 0.54$ & $1.54 \pm 0.35$ & $6.45 \mathrm{E}-15$ & $3.46 \mathrm{E}-14$ & 0.81 \\
\hline 483 & $8: 58: 41.60$ & $-47: 28: 35.59$ & 56.0 & 42.4 & $0.53 \pm 0.28$ & $2.93 \pm 2.92$ & $4.65 \mathrm{E}-15$ & $8.16 \mathrm{E}-15$ & 0.74 \\
\hline 484 & $8: 58: 41.03$ & $-47: 29: 34.14$ & 20.0 & 8.4 & $0.00 \pm 0.05$ & $0.07 \pm 0.01$ & $1.65 \mathrm{E}-14$ & $1.66 \mathrm{E}-14$ & 0.52 \\
\hline 485 & $8: 58: 40.87$ & $-47: 29: 45.46$ & 16.0 & 4.4 & $0.00 \pm 0.14$ & $1.44 \pm \ldots$ & $4.20 \mathrm{E}-16$ & $4.20 \mathrm{E}-16$ & 0.54 \\
\hline 486 & $8: 58: 39.68$ & $-47: 31: 27.60$ & 37.0 & 23.2 & $2.01 \pm 1.42$ & $10.00 \pm \ldots$ & $4.99 \mathrm{E}-15$ & $9.03 \mathrm{E}-15$ & 0.68 \\
\hline 487 & $8: 58: 36.39$ & $-47: 32: 50.72$ & 79.0 & 68.2 & $3.20 \pm 0.68$ & $4.13 \pm 1.90$ & $1.49 \mathrm{E}-14$ & 3.93E-14 & 0.78 \\
\hline 488 & $8: 58: 36.09$ & $-47: 31: 36.23$ & 33.0 & 21.5 & $2.96 \pm 0.93$ & $1.57 \pm 0.55$ & $3.28 \mathrm{E}-15$ & $1.82 \mathrm{E}-14$ & 0.52 \\
\hline 489 & $8: 58: 34.61$ & $-47: 28: 16.33$ & 143.0 & 114.1 & $1.38 \pm 0.28$ & $3.10 \pm 1.08$ & $1.61 \mathrm{E}-14$ & $3.66 \mathrm{E}-14$ & 0.98 \\
\hline 490 & $8: 58: 31.45$ & $-47: 28: 08.65$ & 85.0 & 71.5 & $0.03 \pm 0.10$ & $3.38 \pm 6.64$ & $8.34 \mathrm{E}-15$ & $9.10 \mathrm{E}-15$ & 0.78 \\
\hline 491 & $8: 58: 29.85$ & $-47: 28: 23.50$ & 112.0 & 99.7 & $0.54 \pm 0.17$ & $2.93 \pm 1.23$ & $1.70 \mathrm{E}-14$ & $3.00 \mathrm{E}-14$ & 0.79 \\
\hline 492 & $8: 58: 27.61$ & $-47: 30: 08.56$ & 91.0 & 59.3 & $2.10 \pm 0.44$ & $1.62 \pm 0.47$ & $7.68 \mathrm{E}-15$ & $3.34 \mathrm{E}-14$ & 0.99 \\
\hline 493 & $8: 58: 26.16$ & $-47: 22: 46.53$ & 260.0 & 128.6 & $1.38 \pm 0.18$ & $1.02 \pm 0.17$ & $8.09 \mathrm{E}-15$ & $5.04 \mathrm{E}-14$ & 1.33 \\
\hline 494 & $8: 58: 21.20$ & $-47: 31: 06.55$ & 102.0 & 72.0 & $0.00 \pm 0.09$ & $2.72 \pm 2.95$ & $8.15 \mathrm{E}-15$ & $8.15 \mathrm{E}-15$ & 1.03 \\
\hline 495 & $8: 59: 57.87$ & $-47: 32: 15.05$ & 28.0 & 16.4 & $0.00 \pm 0.37$ & $7.41 \pm \ldots$ & $2.07 \mathrm{E}-15$ & $2.07 \mathrm{E}-15$ & 0.69 \\
\hline
\end{tabular}


Table 2-Continued

\begin{tabular}{|c|c|c|c|c|c|c|c|c|c|}
\hline $\begin{array}{l}\text { Chandra } \\
\text { ID }\end{array}$ & $\begin{array}{c}\text { RA } \\
(2000)\end{array}$ & $\begin{array}{c}\text { Dec } \\
(2000)\end{array}$ & $\begin{array}{l}\text { Raw } \\
\text { CNT }\end{array}$ & $\begin{array}{l}\text { Net }^{a} \\
\text { CNT }\end{array}$ & $\begin{array}{c}N_{H} \\
10^{22} \mathrm{~cm}^{-2}\end{array}$ & $\begin{array}{l}\mathrm{kT} \\
\mathrm{keV}\end{array}$ & \multicolumn{2}{|c|}{$\left[\mathrm{ergcm}^{-2} \mathrm{~s}^{-1}\right]$} & $\chi^{2}$ \\
\hline 496 & $8: 59: 55.90$ & $-47: 35: 45.88$ & 39.0 & 12.7 & $2.68 \pm 1.65$ & $1.94 \pm 1.57$ & $2.44 \mathrm{E}-15$ & $1.01 \mathrm{E}-14$ & 0.78 \\
\hline 497 & $8: 59: 46.86$ & $-47: 38: 04.09$ & 62.0 & 39.9 & $0.05 \pm 0.26$ & $10.00 \pm \cdots$ & $4.63 \mathrm{E}-15$ & $5.00 \mathrm{E}-15$ & 0.95 \\
\hline 498 & $8: 59: 37.86$ & $-47: 23: 03.10$ & 53.0 & 42.4 & $2.10 \pm 0.83$ & $10.00 \pm \ldots$ & $8.84 \mathrm{E}-15$ & $1.61 \mathrm{E}-14$ & 0.81 \\
\hline 499 & $8: 59: 34.18$ & $-47: 38: 35.03$ & 47.0 & 20.3 & $0.00 \pm 0.07$ & $1.62 \pm 8.92$ & $1.88 \mathrm{E}-15$ & $1.88 \mathrm{E}-15$ & 0.87 \\
\hline 500 & $8: 59: 33.20$ & $-47: 20: 45.11$ & 48.0 & 39.6 & $0.00 \pm 0.06$ & $0.97 \pm 0.13$ & $6.91 \mathrm{E}-15$ & $6.91 \mathrm{E}-15$ & 0.64 \\
\hline 501 & $8: 59: 27.63$ & $-47: 37: 13.57$ & 32.0 & 19.2 & $1.53 \pm 0.82$ & $1.60 \pm 1.51$ & $1.85 \mathrm{E}-15$ & $6.82 \mathrm{E}-15$ & 0.73 \\
\hline 502 & $8: 59: 22.15$ & $-47: 40: 05.86$ & 35.0 & 14.9 & $0.00 \pm 0.13$ & $1.14 \pm 0.91$ & $1.53 \mathrm{E}-15$ & $1.53 \mathrm{E}-15$ & 0.92 \\
\hline 503 & $8: 59: 20.70$ & $-47: 38: 13.90$ & 39.0 & 26.3 & $2.96 \pm 1.90$ & $10.00 \pm \ldots$ & $6.07 \mathrm{E}-15$ & $1.21 \mathrm{E}-14$ & 0.70 \\
\hline 504 & $8: 59: 13.86$ & $-47: 19: 07.29$ & 98.0 & 80.3 & $0.00 \pm 0.05$ & $1.34 \mathrm{v} 0.56$ & $1.24 \mathrm{E}-14$ & $1.24 \mathrm{E}-14$ & 0.94 \\
\hline 505 & $8: 59: 13.74$ & $-47: 23: 18.11$ & 42.0 & 29.1 & $7.17 \pm 4.09$ & $10.00 \pm \cdots$ & $8.04 \mathrm{E}-15$ & $2.16 \mathrm{E}-14$ & 0.74 \\
\hline 506 & $8: 59: 08.42$ & $-47: 37: 19.46$ & 33.0 & 22.5 & $3.12 \pm 2.02$ & $4.70 \pm \ldots$ & 4.17E-15 & $1.03 \mathrm{E}-14$ & 0.70 \\
\hline 507 & $8: 58: 59.91$ & $-47: 22: 45.04$ & 62.0 & 41.5 & $0.18 \pm 0.25$ & $5.95 \pm 30.31$ & $5.19 \mathrm{E}-15$ & $6.49 \mathrm{E}-15$ & 0.95 \\
\hline 508 & $8: 58: 57.83$ & $-47: 23: 58.48$ & 39.0 & 24.1 & $0.00 \pm 0.24$ & $4.58 \pm 51.33$ & $3.00 \mathrm{E}-15$ & $3.00 \mathrm{E}-15$ & 0.57 \\
\hline 509 & $8: 58: 49.82$ & $-47: 35: 20.67$ & 27.0 & 15.2 & $3.61 \pm 2.58$ & $3.72 \pm 5.85$ & $3.41 \mathrm{E}-15$ & $9.93 \mathrm{E}-15$ & 0.69 \\
\hline 510 & $8: 58: 44.53$ & $-47: 34: 07.37$ & 44.0 & 31.3 & $1.42 \pm 0.58$ & $3.15 \pm 2.70$ & $4.68 \mathrm{E}-15$ & $1.06 \mathrm{E}-14$ & 0.68 \\
\hline 511 & $8: 58: 43.73$ & $-47: 26: 45.91$ & 34.0 & 18.8 & $2.84 \pm 2.21$ & $10.00 \pm \cdots$ & $4.83 \mathrm{E}-15$ & $9.56 \mathrm{E}-15$ & 0.73 \\
\hline 512 & $8: 58: 43.24$ & $-47: 34: 54.21$ & 27.0 & 16.8 & $7.87 \pm 2.42$ & $1.13 \pm 0.19$ & $3.28 \mathrm{E}-15$ & $7.71 \mathrm{E}-14$ & 0.61 \\
\hline 513 & $8: 58: 41.82$ & $-47: 26: 27.14$ & 42.0 & 29.6 & $3.47 \pm 1.91$ & $5.93 \pm \ldots$ & $6.75 \mathrm{E}-15$ & $1.59 \mathrm{E}-14$ & 0.73 \\
\hline 514 & $8: 58: 38.21$ & $-47: 33: 14.68$ & 24.0 & 13.4 & $1.60 \pm 0.85$ & $1.75 \pm 1.45$ & $1.99 \mathrm{E}-15$ & $6.93 \mathrm{E}-15$ & 0.63 \\
\hline 515 & $8: 58: 33.14$ & $-47: 22: 55.20$ & 374.0 & 332.7 & $0.21 \pm 0.06$ & $10.00 \pm 11.88$ & $3.70 \mathrm{E}-14$ & $4.56 \mathrm{E}-14$ & 1.34 \\
\hline 516 & $8: 58: 33.24$ & $-47: 31: 55.84$ & 57.0 & 29.9 & $2.75 \pm 1.78$ & $10.00 \pm \ldots$ & $6.75 \mathrm{E}-15$ & $1.32 \mathrm{E}-14$ & 0.85 \\
\hline 517 & $8: 58: 30.98$ & $-47: 32: 21.92$ & 54.0 & 28.7 & $0.00 \pm 0.35$ & $10.00 \pm \ldots$ & $3.96 \mathrm{E}-15$ & $3.96 \mathrm{E}-15$ & 0.87 \\
\hline 518 & $8: 58: 23.76$ & $-47: 30: 01.19$ & 63.0 & 51.5 & $1.40 \pm 0.54$ & $6.32 \pm 46.15$ & $8.72 \mathrm{E}-15$ & $1.57 \mathrm{E}-14$ & 0.70 \\
\hline 519 & $8: 58: 16.78$ & $-47: 25: 34.50$ & 435.0 & 413.9 & $0.40 \pm 0.07$ & $10.00 \pm 14.19$ & $5.10 \mathrm{E}-14$ & $6.81 \mathrm{E}-14$ & 0.91 \\
\hline 520 & $8: 58: 37.89$ & $-47: 31: 10.45$ & 33.0 & 20.6 & $2.12 \pm 1.42$ & $10.00 \pm \ldots$ & $4.05 \mathrm{E}-15$ & $7.43 \mathrm{E}-15$ & 0.63 \\
\hline 521 & $8: 58: 29.95$ & $-47: 23: 00.99$ & 315.0 & 246.7 & $0.65 \pm 0.13$ & $5.23 \pm 5.00$ & $2.75 \mathrm{E}-14$ & $4.34 \mathrm{E}-14$ & 1.39 \\
\hline 522 & $8: 58: 22.11$ & $-47: 25: 16.55$ & 181.0 & 155.1 & $0.00 \pm 0.01$ & $10.00 \pm 44.44$ & $1.54 \mathrm{E}-14$ & $1.54 \mathrm{E}-14$ & 1.22 \\
\hline 523 & $8: 58: 20.20$ & $-47: 23: 50.32$ & 485.0 & 250.9 & $0.00 \pm 0.01$ & $8.09 \pm 9.00$ & $2.19 \mathrm{E}-14$ & $2.19 \mathrm{E}-14$ & 1.54 \\
\hline 524 & $8: 58: 20.20$ & $-47: 24: 30.46$ & 305.0 & 216.6 & $0.96 \pm 0.14$ & $2.03 \pm 0.62$ & $1.85 \mathrm{E}-14$ & $4.63 \mathrm{E}-14$ & 1.06 \\
\hline 525 & $8: 58: 12.64$ & $-47: 24: 49.69$ & 688.0 & 397.8 & $1.03 \pm 0.12$ & $2.67 \pm 0.65$ & $4.19 \mathrm{E}-14$ & $9.27 \mathrm{E}-14$ & 1.28 \\
\hline 526 & $8: 58: 12.04$ & $-47: 27: 08.22$ & 252.0 & 242.9 & $1.12 \pm 0.21$ & $10.00 \pm \ldots$ & $3.52 \mathrm{E}-14$ & $5.59 \mathrm{E}-14$ & 1.05 \\
\hline 527 & $8: 58: 08.25$ & $-47: 26: 44.36$ & 412.0 & 333.1 & $0.00 \pm 0.01$ & $10.00 \pm \ldots$ & $2.44 \mathrm{E}-14$ & $2.44 \mathrm{E}-14$ & 1.53 \\
\hline 528 & $8: 58: 05.61$ & $-47: 28: 00.18$ & 382.0 & 354.8 & $0.00 \pm 0.00$ & $10.00 \pm \ldots$ & $3.06 \mathrm{E}-14$ & $3.06 \mathrm{E}-14$ & 1.64 \\
\hline 529 & $8: 58: 51.65$ & $-47: 16: 22.86$ & 493.0 & 311.2 & $0.00 \pm 0.01$ & $1.00 \pm 0.06$ & $2.15 \mathrm{E}-14$ & $2.15 \mathrm{E}-14$ & 1.04 \\
\hline 530 & $8: 58: 25.27$ & $-47: 20: 47.70$ & 389.0 & 196.0 & $0.98 \pm 0.14$ & $1.25 \pm 0.30$ & 1.10E-14 & $4.00 \mathrm{E}-14$ & 1.52 \\
\hline 531 & $8: 58: 06.70$ & $-47: 20: 31.67$ & 704.0 & 330.9 & $0.00 \pm 0.00$ & $1.08 \pm \ldots$ & $1.46 \mathrm{E}-14$ & $1.46 \mathrm{E}-14$ & 1.73 \\
\hline 532 & $8: 58: 05.07$ & $-47: 19: 04.06$ & 559.0 & 223.7 & $0.37 \pm 0.17$ & $10.00 \pm 26.54$ & 2.33E-14 & $3.08 \mathrm{E}-14$ & 1.48 \\
\hline 533 & $8: 58: 04.36$ & $-47: 23: 02.24$ & 1333.0 & 846.4 & $1.62 \pm 0.10$ & $2.77 \pm 0.34$ & $1.28 \mathrm{E}-13$ & $3.25 \mathrm{E}-13$ & 2.36 \\
\hline 534 & $8: 58: 44.37$ & $-47: 14: 43.52$ & 364.0 & 194.3 & $1.24 \pm 0.27$ & $5.69 \pm 7.74$ & $3.68 \mathrm{E}-14$ & $6.56 \mathrm{E}-14$ & 1.21 \\
\hline 535 & $8: 58: 02.94$ & $-47: 25: 17.86$ & 669.0 & 378.3 & $0.00 \pm 0.00$ & $10.00 \pm 17.77$ & $2.81 \mathrm{E}-14$ & $2.81 \mathrm{E}-14$ & 2.01 \\
\hline 536 & $8: 58: 14.31$ & $-47: 16: 11.21$ & 306.0 & 121.7 & $0.00 \pm 0.02$ & $0.85 \pm 0.09$ & $8.08 \mathrm{E}-15$ & 8.09E-15 & 1.66 \\
\hline
\end{tabular}

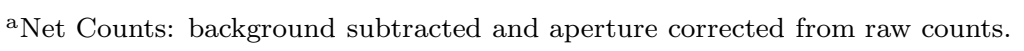

${ }^{b}$ Abs. Flux: Absorbed flux. Tot. UnAbs. Flux: Total unabsorbed flux. 
Table 3. Astrometry of the Class 0/I YSOs in RCW 38.

\begin{tabular}{rrrrrr}
\hline \hline Spitzer ID & Epoch ID & RA (J2000) & Dec $(\mathrm{J} 2000)$ & Class & X-ray, Variable $^{\mathrm{a}}$ \\
\hline 72 & 3 & $8: 59: 05.86$ & $-47: 36: 29.41$ & $0 / \mathrm{I}$ & $\ldots$ \\
114 & 3 & $8: 58: 59.19$ & $-47: 32: 32.86$ & $0 / \mathrm{I}$ & $\mathrm{x}$ \\
138 & 3 & $8: 59: 11.11$ & $-47: 31: 23.85$ & $0 / \mathrm{I}$ & $\mathrm{x}$ \\
141 & 3 & $8: 59: 00.64$ & $-47: 27: 24.65$ & $0 / \mathrm{I}$ & $\mathrm{x}, \mathrm{v}$ \\
165 & 3 & $8: 58: 25.45$ & $-47: 31: 34.96$ & $0 / \mathrm{I}$ & $\mathrm{v}$ \\
185 & 3 & $8: 59: 16.56$ & $-47: 30: 25.30$ & $0 / \mathrm{I}$ & $\cdots$ \\
239 & 3 & $8: 59: 27.93$ & $-47: 32: 56.47$ & $0 / \mathrm{I}$ & $\cdots$ \\
258 & 3 & $8: 59: 09.31$ & $-47: 29: 18.92$ & $0 / \mathrm{I}$ & $\mathrm{x}$ \\
270 & 3 & $8: 58: 25.59$ & $-47: 30: 51.43$ & $0 / \mathrm{I}$ & $\mathrm{v}$ \\
274 & 1 & $8: 59: 10.63$ & $-47: 22: 39.05$ & $0 / \mathrm{I}$ & $\mathrm{x}, \mathrm{v}$ \\
291 & 3 & $8: 59: 15.30$ & $-47: 27: 05.79$ & $0 / \mathrm{I}$ & $\cdots$ \\
292 & 3 & $8: 58: 39.39$ & $-47: 21: 11.58$ & $0 / \mathrm{I}$ & $\cdots$ \\
453 & 1 & $8: 59: 48.22$ & $-47: 17: 57.92$ & $0 / \mathrm{I}$ & $\cdots$ \\
472 & 4 & $8: 59: 01.28$ & $-47: 26: 05.38$ & $0 / \mathrm{I}$ & $\cdots$ \\
475 & 4 & $8: 59: 09.97$ & $-47: 26: 09.38$ & $0 / \mathrm{I}$ & $\cdots$ \\
478 & 3 & $8: 59: 13.78$ & $-47: 29: 05.75$ & $0 / \mathrm{I}$ & $\mathrm{x}$ \\
492 & 3 & $8: 59: 05.95$ & $-47: 31: 51.48$ & $0 / \mathrm{I}$ & $\mathrm{x}$ \\
493 & 3 & $8: 59: 15.68$ & $-47: 30: 9.78$ & $0 / \mathrm{I}$ & $\mathrm{v}$ \\
559 & 3 & $8: 58: 23.85$ & $-47: 32: 40.80$ & $0 / \mathrm{I}$ & $\mathrm{v}$ \\
571 & 3 & $8: 58: 46.03$ & $-47: 34: 47.68$ & $0 / \mathrm{I}$ & $\mathrm{x}$ \\
577 & 3 & $8: 59: 06.29$ & $-47: 31: 07.65$ & $0 / \mathrm{I}$ & $\mathrm{x}, \mathrm{v}$ \\
581 & 3 & $8: 59: 02.92$ & $-47: 30: 54.34$ & $0 / \mathrm{I}$ & \\
589 & 3 & $8: 59: 03.00$ & $-47: 30: 44.50$ & $0 / \mathrm{I}$ & \\
& & & & & \\
\hline & & & & \\
\hline
\end{tabular}

${ }^{a} \mathrm{v}$ : indicates source is a candidate variable. 
Table 4. Astrometry of the flat spectrum YSOs in RCW 38.

\begin{tabular}{|c|c|c|c|c|c|}
\hline Spitzer ID & Epoch ID & RA (J2000) & Dec (J2000) & Class & $\mathrm{X}$-ray, Variable ${ }^{\mathrm{a}}$ \\
\hline 19 & 2 & $8: 58: 39.71$ & $-47: 36: 58.28$ & FS & $\ldots$ \\
\hline 26 & 4 & $8: 58: 41.82$ & $-47: 38: 22.66$ & $\mathrm{FS}$ & $\cdots$ \\
\hline 42 & 2 & $8: 58: 25.91$ & $-47: 36: 34.24$ & FS & $\cdots$ \\
\hline 52 & 3 & $8: 59: 30.12$ & $-47: 38: 39.42$ & $\mathrm{FS}$ & $\ldots$ \\
\hline 64 & 2 & $8: 58: 45.67$ & $-47: 38: 24.67$ & FS & $\cdots$ \\
\hline 69 & 2 & $8: 59: 37.74$ & $-47: 38: 43.84$ & FS & $\ldots$ \\
\hline 78 & 2 & $8: 58: 35.16$ & $-47: 40: 09.08$ & FS & $\cdots$ \\
\hline 80 & 4 & $8: 58: 29.70$ & $-47: 38: 18.43$ & FS & $\ldots$ \\
\hline 84 & 2 & 9:00:02.26 & $-47: 36: 31.24$ & FS & $\ldots$ \\
\hline 85 & 4 & $8: 58: 30.06$ & $-47: 37: 41.95$ & FS & $\mathrm{v}$ \\
\hline 86 & 4 & $8: 59: 54.11$ & $-47: 36: 20.97$ & FS & $\cdots$ \\
\hline 92 & 4 & $8: 58: 08.70$ & $-47: 25: 04.40$ & FS & $\cdots$ \\
\hline 95 & 3 & $8: 58: 41.19$ & $-47: 31: 04.24$ & FS & $\ldots$ \\
\hline 97 & 1 & $8: 58: 01.21$ & $-47: 23: 36.75$ & FS & $\mathrm{v}$ \\
\hline 98 & 3 & $8: 59: 00.64$ & $-47: 32: 08.51$ & FS & $\ldots$ \\
\hline 106 & 3 & $8: 59: 13.95$ & $-47: 27: 43.51$ & FS & $\cdots$ \\
\hline 116 & 3 & $8: 59: 15.78$ & $-47: 27: 01.18$ & FS & $\mathrm{x}, \mathrm{v}$ \\
\hline 126 & 1 & $8: 58: 04.20$ & $-47: 23: 10.00$ & FS & $\ldots$ \\
\hline 144 & 3 & $8: 59: 00.31$ & $-47: 31: 14.50$ & FS & $\mathrm{x}$ \\
\hline 153 & 1 & $8: 58: 20.73$ & $-47: 23: 29.84$ & FS & $\ldots$ \\
\hline 156 & 3 & $8: 59: 09.72$ & $-47: 28: 45.36$ & $\mathrm{FS}$ & $\mathrm{x}$ \\
\hline 164 & 3 & $8: 59: 02.24$ & $-47: 26: 39.20$ & $\mathrm{FS}$ & $\mathrm{x}, \mathrm{v}$ \\
\hline 173 & 4 & $8: 57: 56.64$ & $-47: 30: 59.89$ & FS & $\ldots$ \\
\hline 174 & 3 & $8: 59: 18.21$ & $-47: 28: 54.43$ & FS & $\ldots$ \\
\hline 181 & 3 & $8: 59: 31.27$ & $-47: 33: 18.14$ & FS & $\ldots$ \\
\hline 182 & 3 & $8: 58: 57.58$ & $-47: 24: 26.07$ & FS & $\mathrm{x}$ \\
\hline 183 & 3 & $8: 59: 04.53$ & $-47: 25: 57.78$ & FS & $\ldots$ \\
\hline 187 & 3 & $8: 58: 58.14$ & $-47: 26: 55.77$ & FS & $\ldots$ \\
\hline 193 & 2 & $8: 58: 26.71$ & $-47: 23: 37.68$ & FS & $\cdots$ \\
\hline 198 & 3 & $8: 59: 16.40$ & $-47: 28: 25.85$ & FS & $\cdots$ \\
\hline 201 & 3 & $8: 58: 26.09$ & $-47: 27: 23.65$ & FS & $\ldots$ \\
\hline 203 & 3 & $8: 59: 40.25$ & $-47: 30: 27.70$ & FS & $\mathrm{v}$ \\
\hline 207 & 1 & $8: 58: 19.28$ & $-47: 22: 28.94$ & FS & $\mathrm{v}$ \\
\hline 208 & 3 & $8: 58: 58.74$ & $-47: 31: 04.32$ & FS & $\mathrm{x}$ \\
\hline 214 & 4 & $8: 58: 22.90$ & $-47: 28: 14.85$ & FS & $\ldots$ \\
\hline 215 & 3 & $8: 58: 53.48$ & $-47: 32: 07.11$ & FS & $\ldots$ \\
\hline 220 & 3 & $8: 58: 25.31$ & $-47: 30: 53.74$ & FS & $\cdots$ \\
\hline 227 & 3 & $8: 58: 32.69$ & $-47: 31: 18.69$ & FS & $\mathrm{v}$ \\
\hline 230 & 4 & $8: 59: 03.70$ & $-47: 31: 50.81$ & FS & $\cdots$ \\
\hline 240 & 4 & $8: 57: 57.13$ & $-47: 30: 18.53$ & $\mathrm{FS}$ & $\ldots$ \\
\hline 260 & 3 & $8: 58: 32.82$ & $-47: 26: 58.37$ & FS & $\cdots$ \\
\hline 266 & 4 & $8: 58: 28.16$ & $-47: 24: 33.64$ & $\mathrm{FS}$ & $\mathrm{v}$ \\
\hline 271 & 3 & $8: 59: 24.35$ & $-47: 30: 44.34$ & FS & $\mathrm{x}$ \\
\hline 306 & 3 & $8: 58: 19.87$ & $-47: 32: 27.01$ & FS & $\ldots$ \\
\hline 316 & 1 & $8: 58: 05.52$ & $-47: 28: 13.50$ & FS & $\ldots$ \\
\hline 324 & 3 & $8: 59: 16.77$ & $-47: 27: 54.69$ & FS & $\ldots$ \\
\hline
\end{tabular}


Table 4-Continued

\begin{tabular}{|c|c|c|c|c|c|}
\hline Spitzer ID & Epoch ID & RA (J2000) & $\operatorname{Dec}(\mathrm{J} 2000)$ & Class & X-ray, Variable ${ }^{a}$ \\
\hline 325 & 3 & 8:59:07.09 & $-47: 31: 49.93$ & FS & $\mathrm{x}$ \\
\hline 344 & 1 & $8: 58: 26.99$ & $-47: 23: 08.13$ & FS & $\ldots$ \\
\hline 346 & 4 & $8: 59: 16.68$ & $-47: 29: 39.40$ & FS & $\ldots$ \\
\hline 351 & 3 & $8: 59: 12.62$ & $-47: 30: 07.43$ & FS & $\cdots$ \\
\hline 352 & 3 & $8: 59: 04.23$ & $-47: 23: 21.94$ & FS & $\mathrm{x}$ \\
\hline 353 & 3 & $8: 59: 20.29$ & $-47: 31: 06.11$ & FS & $\mathrm{x}$ \\
\hline 356 & 2 & $8: 58: 58.24$ & $-47: 23: 29.90$ & FS & $\ldots$ \\
\hline 362 & 3 & $8: 58: 43.04$ & $-47: 32: 04.61$ & FS & $\cdots$ \\
\hline 381 & 3 & 8:59:19.64 & $-47: 30: 59.62$ & FS & $\ldots$ \\
\hline 382 & 3 & $8: 58: 29.85$ & $-47: 28: 23.04$ & FS & $\mathrm{x}$ \\
\hline 388 & 3 & $8: 59: 18.97$ & $-47: 32: 24.93$ & FS & $\mathrm{x}$ \\
\hline 396 & 3 & $8: 58: 44.09$ & $-47: 33: 23.98$ & FS & $\ldots$ \\
\hline 403 & 2 & $8: 58: 03.24$ & $-47: 22: 56.48$ & FS & $\ldots$ \\
\hline 404 & 3 & $8: 58: 43.59$ & $-47: 31: 18.57$ & FS & $\mathrm{v}$ \\
\hline 414 & 3 & 8:59:06.84 & $-47: 29: 41.29$ & FS & $\mathrm{x}$ \\
\hline 434 & 1 & 9:00:15.50 & $-47: 26: 24.67$ & $\mathrm{FS}$ & $\ldots$ \\
\hline 438 & 1 & $8: 59: 11.86$ & $-47: 14: 35.83$ & FS & $\ldots$ \\
\hline 443 & 1 & $8: 59: 27.89$ & $-47: 15: 37.31$ & FS & $\ldots$ \\
\hline 459 & 3 & $8: 59: 42.59$ & $-47: 26: 29.04$ & FS & $\ldots$ \\
\hline 460 & 3 & $8: 59: 32.12$ & $-47: 18: 09.27$ & FS & $\ldots$ \\
\hline 466 & 1 & $8: 58: 05.67$ & $-47: 19: 16.20$ & FS & $\ldots$ \\
\hline 467 & 1 & $8: 58: 23.66$ & $-47: 30: 03.36$ & FS & $\ldots$ \\
\hline 471 & 2 & $8: 58: 33.09$ & $-47: 38: 37.15$ & FS & $\ldots$ \\
\hline 477 & 3 & $8: 59: 14.58$ & $-47: 26: 49.77$ & FS & $\mathrm{x}$ \\
\hline 481 & 1 & $8: 59: 46.26$ & $-47: 17: 35.11$ & FS & $\ldots$ \\
\hline 490 & 3 & $8: 58: 53.67$ & $-47: 30: 14.44$ & FS & $\mathrm{x}, \mathrm{v}$ \\
\hline 502 & 2 & $8: 58: 11.81$ & $-47: 42: 49.92$ & FS & $\ldots$ \\
\hline 505 & 3 & $8: 59: 25.50$ & $-47: 39: 59.48$ & FS & $\ldots$ \\
\hline 516 & 3 & $8: 58: 45.61$ & $-47: 34: 52.50$ & FS & $\mathrm{v}$ \\
\hline 524 & 2 & $8: 58: 49.11$ & $-47: 46: 25.19$ & FS & $\ldots$ \\
\hline 531 & 2 & 8:58:08.99 & $-47: 42: 08.09$ & FS & $\cdots$ \\
\hline 536 & 2 & $9: 0: 10.77$ & $-47: 39: 49.06$ & FS & $\ldots$ \\
\hline 543 & 3 & $8: 58: 49.15$ & $-47: 34: 09.14$ & FS & $\mathrm{x}$ \\
\hline 561 & 3 & $8: 58: 58.05$ & $-47: 32: 44.00$ & FS & $\ldots$ \\
\hline 572 & 4 & $8: 58: 23.59$ & $-47: 30: 04.14$ & FS & $\cdots$ \\
\hline 573 & 3 & $8: 58: 55.64$ & $-47: 35: 49.67$ & FS & $\ldots$ \\
\hline 585 & 3 & $8: 59: 14.00$ & $-47: 28: 56.50$ & FS & $\mathrm{x}, \mathrm{v}$ \\
\hline 590 & 3 & $8: 59: 16.88$ & $-47: 27: 48.28$ & FS & $\mathrm{x}$ \\
\hline 598 & 2 & $8: 58: 26.17$ & $-47: 30: 41.48$ & FS & $\ldots$ \\
\hline 756 & 1 & $8: 58: 07.52$ & $-47: 24: 41.75$ & FS & $\ldots$ \\
\hline 762 & 1 & $8: 58: 38.79$ & $-47: 16: 32.73$ & FS & $\ldots$ \\
\hline 774 & 4 & $8: 59: 15.66$ & $-47: 25: 35.42$ & FS & $\ldots$ \\
\hline 813 & 4 & 8:59:07.01 & $-47: 30: 23.07$ & FS & $\ldots$ \\
\hline 814 & 4 & 8:59:7.93 & $-47: 30: 34.00$ & FS & $\cdots$ \\
\hline
\end{tabular}

$\mathrm{a}_{\mathrm{x}}$ : indicates Chandra X-ray detection. v: indicates source is a candidate variable. 
Table 5. Astrometry of the Class II YSOs in RCW 38.

\begin{tabular}{|c|c|c|c|c|c|}
\hline Spitzer ID & Epoch ID & RA (J2000) & Dec (J2000) & Class & $\mathrm{X}$-ray, Var., O? ${ }^{\mathrm{a}}$ \\
\hline 1 & 4 & $8: 58: 40.06$ & $-47: 37: 49.64$ & II & $\ldots$ \\
\hline 2 & 3 & $8: 59: 08.45$ & $-47: 37: 19.47$ & II & $\mathrm{x}$ \\
\hline 3 & 3 & $8: 59: 41.57$ & $-47: 35: 07.99$ & II & $\mathrm{x}$ \\
\hline 4 & 4 & $8: 57: 59.98$ & $-47: 36: 02.43$ & II & $\cdots$ \\
\hline 5 & 2 & $8: 59: 31.08$ & $-47: 41: 36.04$ & II & $\ldots$ \\
\hline 6 & 4 & $8: 58: 00.31$ & $-47: 36: 47.36$ & II & $\ldots$ \\
\hline 7 & 3 & $8: 58: 42.75$ & $-47: 35: 13.33$ & II & $\ldots$ \\
\hline 8 & 2 & $8: 59: 11.80$ & $-47: 40: 43.99$ & II & $\cdots$ \\
\hline 9 & 4 & $8: 57: 54.40$ & $-47: 36: 33.98$ & II & $\ldots$ \\
\hline 10 & 2 & $8: 59: 32.57$ & $-47: 41: 11.99$ & II & $\mathrm{v}$ \\
\hline 11 & 4 & $8: 58: 12.59$ & $-47: 37: 53.39$ & II & $\ldots$ \\
\hline 12 & 3 & $8: 58: 15.06$ & $-47: 34: 52.99$ & II & $\ldots$ \\
\hline 13 & 2 & $8: 58: 18.11$ & $-47: 39: 06.99$ & II & $\cdots$ \\
\hline 14 & 4 & $8: 57: 56.33$ & $-47: 35: 27.23$ & II & $\ldots$ \\
\hline 15 & 2 & $8: 59: 21.36$ & $-47: 40: 31.48$ & II & $\ldots$ \\
\hline 16 & 4 & $8: 59: 47.53$ & $-47: 36: 34.16$ & II & $\ldots$ \\
\hline 18 & 4 & $8: 58: 09.54$ & $-47: 37: 52.94$ & II & $\ldots$ \\
\hline 20 & 2 & $8: 58: 52.62$ & $-47: 39: 39.07$ & II & $\ldots$ \\
\hline 21 & 2 & $8: 59: 02.15$ & $-47: 39: 33.18$ & II & $\ldots$ \\
\hline 22 & 3 & 8:59:01.19 & $-47: 37: 54.14$ & II & $\ldots$ \\
\hline 23 & 2 & $8: 58: 50.96$ & $-47: 40: 44.83$ & II & $\ldots$ \\
\hline 24 & 3 & $8: 59: 18.37$ & $-47: 37: 42.51$ & II & $\ldots$ \\
\hline 25 & 2 & $8: 58: 26.41$ & $-47: 39: 24.26$ & II & $\ldots$ \\
\hline 27 & 4 & $8: 58: 07.88$ & $-47: 36: 09.41$ & II & $\ldots$ \\
\hline 28 & 3 & $8: 58: 34.39$ & $-47: 36: 31.87$ & II & $\mathrm{v}$ \\
\hline 29 & 3 & $8: 59: 38.24$ & $-47: 37: 09.89$ & II & $\cdots$ \\
\hline 30 & 3 & $8: 58: 50.56$ & $-47: 36: 36.43$ & II & $\ldots$ \\
\hline 31 & 3 & $8: 59: 23.59$ & $-47: 37: 50.85$ & II & $\mathrm{x}, \mathrm{v}$ \\
\hline 32 & 3 & $8: 59: 24.73$ & $-47: 38: 29.03$ & II & $\mathrm{x}, \mathrm{v}$ \\
\hline 33 & 1 & $8: 59: 28.14$ & $-47: 34: 20.49$ & II & $\cdots$ \\
\hline 34 & 2 & $8: 57: 38.17$ & $-47: 37: 15.29$ & II & $\ldots$ \\
\hline 35 & 2 & $8: 58: 19.78$ & $-47: 39: 08.93$ & II & $\ldots$ \\
\hline 37 & 3 & 8:59:07.64 & $-47: 38: 23.12$ & II & $\mathrm{v}$ \\
\hline 38 & 2 & $8: 58: 56.96$ & $-47: 40: 28.44$ & II & $\mathrm{v}$ \\
\hline 39 & 3 & $8: 59: 30.61$ & $-47: 39: 40.99$ & II & $\cdots$ \\
\hline 40 & 2 & $8: 58: 29.21$ & $-47: 39: 51.57$ & II & $\mathrm{v}$ \\
\hline 41 & 3 & $8: 58: 20.42$ & $-47: 35: 27.64$ & II & $\cdots$ \\
\hline 43 & 4 & $8: 58: 07.37$ & $-47: 37: 26.29$ & II & $\ldots$ \\
\hline 44 & 3 & $8: 58: 55.49$ & $-47: 36: 01.11$ & II & $\cdots$ \\
\hline 45 & 2 & $8: 59: 41.98$ & $-47: 38: 45.96$ & II & $\ldots$ \\
\hline 46 & 3 & $8: 59: 24.70$ & $-47: 38: 02.86$ & II & $\mathrm{x}$ \\
\hline 47 & 2 & $8: 58: 27.99$ & $-47: 39: 50.23$ & II & $\ldots$ \\
\hline 48 & 3 & $8: 58: 22.69$ & $-47: 35: 46.13$ & II & $\ldots$ \\
\hline 49 & 3 & $8: 59: 18.44$ & $-47: 35: 51.89$ & II & $\ldots$ \\
\hline 50 & 4 & $8: 58: 14.75$ & $-47: 36: 43.57$ & II & $\ldots$ \\
\hline 51 & 4 & 8:59:09.36 & $-47: 34: 57.59$ & II & $\mathrm{x}$ \\
\hline
\end{tabular}


Table 5-Continued

\begin{tabular}{|c|c|c|c|c|c|}
\hline Spitzer ID & Epoch ID & RA (J2000) & Dec (J2000) & Class & X-ray, Var., O? ${ }^{a}$ \\
\hline 53 & 2 & $8: 59: 23.81$ & $-47: 38: 33.69$ & II & $\cdots$ \\
\hline 54 & 3 & $8: 59: 27.41$ & $-47: 37: 11.90$ & II & $\ldots$ \\
\hline 55 & 3 & $8: 58: 40.61$ & $-47: 36: 23.70$ & II & $\mathrm{v}$ \\
\hline 56 & 2 & $8: 58: 51.82$ & $-47: 40: 41.51$ & II & $\ldots$ \\
\hline 57 & 2 & $8: 58: 32.48$ & $-47: 39: 29.11$ & II & $\cdots$ \\
\hline 58 & 4 & $8: 58: 32.39$ & $-47: 36: 25.48$ & II & $\cdots$ \\
\hline 59 & 4 & $8: 58: 23.37$ & $-47: 36: 53.73$ & II & $\ldots$ \\
\hline 60 & 4 & $8: 59: 41.14$ & $-47: 36: 36.31$ & II & $\cdots$ \\
\hline 61 & 2 & $8: 58: 23.51$ & $-47: 39: 31.56$ & II & $\ldots$ \\
\hline 62 & 2 & $8: 59: 38.78$ & $-47: 39: 35.82$ & II & $\mathrm{v}$ \\
\hline 63 & 3 & $8: 59: 23.54$ & $-47: 35: 51.66$ & II & $\mathrm{x}$ \\
\hline 66 & 4 & $8: 57: 51.93$ & $-47: 34: 09.97$ & II & $\cdots$ \\
\hline 67 & 3 & $8: 58: 31.58$ & $-47: 35: 35.00$ & II & $\cdots$ \\
\hline 68 & 4 & $8: 58: 22.96$ & $-47: 38: 20.88$ & II & $\cdots$ \\
\hline 70 & 2 & $8: 58: 30.00$ & $-47: 39: 51.15$ & II & $\cdots$ \\
\hline 71 & 2 & $8: 58: 51.30$ & $-47: 40: 24.87$ & II & $\ldots$ \\
\hline 73 & 2 & $8: 58: 56.01$ & $-47: 39: 43.02$ & II & $\cdots$ \\
\hline 74 & 3 & $8: 58: 47.53$ & $-47: 37: 18.07$ & II & $\ldots$ \\
\hline 75 & 4 & $8: 58: 25.49$ & $-47: 38: 20.94$ & II & $\cdots$ \\
\hline 76 & 3 & $8: 59: 31.52$ & $-47: 37: 24.38$ & II & $\ldots$ \\
\hline 79 & 1 & $8: 59: 55.26$ & $-47: 37: 19.95$ & II & $\mathrm{x}$ \\
\hline 81 & 4 & $8: 58: 10.93$ & $-47: 36: 57.82$ & II & $\ldots$ \\
\hline 83 & 3 & $8: 59: 26.79$ & $-47: 38: 37.90$ & II & $\cdots$ \\
\hline 87 & 2 & $8: 59: 55.18$ & $-47: 40: 23.37$ & II & $\ldots$ \\
\hline 88 & 4 & $8: 59: 47.81$ & $-47: 37: 34.40$ & II & $\ldots$ \\
\hline 89 & 3 & $8: 59: 17.22$ & $-47: 27: 56.83$ & II & $\mathrm{x}$ \\
\hline 90 & 2 & $8: 59: 50.73$ & $-47: 40: 24.78$ & II & $\cdots$ \\
\hline 91 & 1 & $8: 59: 24.41$ & $-47: 26: 57.64$ & II & $\ldots$ \\
\hline 94 & 4 & $8: 59: 19.54$ & $-47: 27: 53.74$ & II & $\ldots$ \\
\hline 96 & 2 & $8: 59: 42.35$ & $-47: 38: 59.44$ & II & $\cdots$ \\
\hline 101 & 1 & $8: 59: 51.94$ & $-47: 37: 58.62$ & II & $\ldots$ \\
\hline 102 & 3 & $8: 59: 19.58$ & $-47: 33: 28.49$ & II & $\mathrm{x}$ \\
\hline 103 & 3 & $8: 58: 43.88$ & $-47: 28: 56.86$ & II & $\mathrm{x}, \mathrm{v}$ \\
\hline 104 & 3 & $8: 58: 23.82$ & $-47: 30: 01.65$ & II & $\mathrm{x}$ \\
\hline 105 & 3 & $8: 58: 59.08$ & $-47: 27: 47.78$ & II & $\ldots$ \\
\hline 107 & 1 & $8: 57: 48.10$ & $-47: 22: 24.33$ & II & $\cdots$ \\
\hline 108 & 1 & $8: 58: 10.77$ & $-47: 23: 14.10$ & II & $\ldots$ \\
\hline 110 & 4 & $8: 58: 12.07$ & $-47: 25: 02.96$ & II & $\ldots$ \\
\hline 111 & 4 & $8: 59: 21.14$ & $-47: 29: 36.31$ & II & $\cdots$ \\
\hline 112 & 3 & $8: 59: 20.92$ & $-47: 31: 19.32$ & II & $\mathrm{x}$ \\
\hline 113 & 4 & $8: 58: 22.21$ & $-47: 24: 11.80$ & II & $\cdots$ \\
\hline 115 & 3 & $8: 59: 00.05$ & $-47: 29: 57.39$ & II & $\ldots$ \\
\hline 117 & 3 & $8: 59: 01.51$ & $-47: 23: 24.51$ & II & $\mathrm{x}, \mathrm{v}$ \\
\hline 118 & 3 & $8: 58: 35.85$ & $-47: 26: 27.87$ & II & $\ldots$ \\
\hline 119 & 3 & $8: 59: 15.91$ & $-47: 32: 57.51$ & II & $\mathrm{x}$ \\
\hline 120 & 3 & $8: 58: 59.97$ & $-47: 22: 44.50$ & II & $\mathrm{x}$ \\
\hline
\end{tabular}


Table 5-Continued

\begin{tabular}{|c|c|c|c|c|c|}
\hline Spitzer ID & Epoch ID & RA (J2000) & Dec (J2000) & Class & X-ray, Var., O? ${ }^{\text {a }}$ \\
\hline 121 & 3 & $8: 58: 51.37$ & $-47: 27: 22.21$ & II & $\mathrm{x}$ \\
\hline 122 & 1 & $8: 57: 38.52$ & $-47: 30: 31.67$ & II & $\ldots$ \\
\hline 124 & 3 & $8: 59: 03.76$ & $-47: 24: 42.67$ & II & $\cdots$ \\
\hline 127 & 3 & $8: 58: 54.49$ & $-47: 24: 29.39$ & II & $\mathrm{x}, \mathrm{v}$ \\
\hline 128 & 3 & $8: 58: 57.47$ & $-47: 27: 44.22$ & II & $\mathrm{x}$ \\
\hline 130 & 3 & $8: 58: 31.48$ & $-47: 25: 49.70$ & II & $\cdots$ \\
\hline 131 & 3 & $8: 58: 57.53$ & $-47: 27: 14.72$ & II & $\ldots$ \\
\hline 132 & 3 & $8: 59: 02.07$ & $-47: 32: 09.42$ & II & $\mathrm{x}$ \\
\hline 134 & 3 & $8: 59: 31.51$ & $-47: 33: 50.36$ & II & $\mathrm{x}$ \\
\hline 135 & 3 & $8: 58: 47.13$ & $-47: 28: 44.22$ & II & $\cdots$ \\
\hline 136 & 3 & $8: 59: 17.74$ & $-47: 28: 51.45$ & II & $\mathrm{x}$ \\
\hline 137 & 3 & $8: 59: 22.68$ & $-47: 26: 11.59$ & II & $\mathrm{x}$ \\
\hline 139 & 3 & $8: 59: 03.47$ & $-47: 24: 28.12$ & II & $\mathrm{x}$ \\
\hline 140 & 2 & $8: 58: 21.14$ & $-47: 22: 28.16$ & II & $\cdots$ \\
\hline 142 & 3 & $8: 59: 46.73$ & $-47: 31: 53.48$ & II & $\mathrm{x}$ \\
\hline 143 & 3 & $8: 59: 10.86$ & $-47: 32: 14.14$ & II & $\ldots$ \\
\hline 145 & 3 & $8: 58: 20.18$ & $-47: 33: 17.64$ & II & $\cdots$ \\
\hline 146 & 3 & $8: 58: 50.83$ & $-47: 30: 12.91$ & II & $\mathrm{x}$ \\
\hline 147 & 3 & $8: 59: 31.26$ & $-47: 33: 30.43$ & II & $\mathrm{x}$ \\
\hline 148 & 3 & $8: 59: 28.17$ & $-47: 29: 26.02$ & II & $\mathrm{x}$ \\
\hline 149 & 3 & $8: 58: 48.63$ & $-47: 32: 21.39$ & II & $\mathrm{x}$ \\
\hline 150 & 3 & $8: 58: 39.69$ & $-47: 31: 27.12$ & II & $\mathrm{x}$ \\
\hline 151 & 1 & $8: 58: 10.98$ & $-47: 22: 48.39$ & II & \\
\hline 152 & 3 & $8: 58: 59.16$ & $-47: 26: 58.15$ & II & $\mathrm{x}$ \\
\hline 154 & 2 & $8: 58: 53.43$ & $-47: 25: 25.00$ & II & $\mathrm{v}$ \\
\hline 155 & 3 & $8: 59: 25.95$ & $-47: 27: 26.32$ & II & $\mathrm{x}$ \\
\hline 157 & 3 & $8: 58: 59.95$ & $-47: 29: 47.56$ & II & $\mathrm{x}$ \\
\hline 158 & 3 & $8: 58: 59.82$ & $-47: 32: 22.82$ & II & $\mathrm{x}, \mathrm{O}$ \\
\hline 159 & 3 & $8: 58: 49.20$ & $-47: 31: 15.12$ & II & $\mathrm{x}$ \\
\hline 160 & 3 & $8: 59: 29.51$ & $-47: 28: 21.52$ & II & $\ldots$ \\
\hline 161 & 2 & $8: 58: 01.75$ & $-47: 23: 51.93$ & II & $\ldots$ \\
\hline 162 & 3 & $8: 58: 55.07$ & $-47: 25: 02.12$ & II & $\mathrm{x}$ \\
\hline 163 & 1 & $8: 58: 05.56$ & $-47: 29: 33.13$ & II & $\cdots$ \\
\hline 167 & 4 & $8: 58: 26.50$ & $-47: 26: 44.54$ & II & $\ldots$ \\
\hline 168 & 4 & $8: 59: 14.74$ & $-47: 31: 47.36$ & II & $\mathrm{x}$ \\
\hline 170 & 3 & $8: 59: 37.45$ & $-47: 31: 25.63$ & II & $\mathrm{x}$ \\
\hline 171 & 3 & $8: 58: 53.91$ & $-47: 30: 51.39$ & II & $\mathrm{x}$ \\
\hline 172 & 3 & $8: 59: 19.83$ & $-47: 27: 31.19$ & II & $\mathrm{x}$ \\
\hline 175 & 3 & $8: 59: 22.51$ & $-47: 29: 04.07$ & II & $\ldots$ \\
\hline 176 & 3 & $8: 58: 49.74$ & $-47: 28: 10.35$ & II & $\ldots$ \\
\hline 177 & 4 & $8: 59: 26.85$ & $-47: 29: 40.29$ & II & $\ldots$ \\
\hline 178 & 3 & $8: 59: 21.92$ & $-47: 30: 38.33$ & II & $\mathrm{x}$ \\
\hline 179 & 4 & $8: 58: 22.89$ & $-47: 25: 38.58$ & II & $\ldots$ \\
\hline 180 & 1 & $8: 58: 08.41$ & $-47: 26: 05.88$ & II & $\cdots$ \\
\hline 184 & 1 & $8: 58: 00.83$ & $-47: 23: 17.64$ & II & $\ldots$ \\
\hline 186 & 3 & $8: 58: 58.31$ & $-47: 27: 27.62$ & II & $\mathrm{x}$ \\
\hline
\end{tabular}


Table 5-Continued

\begin{tabular}{|c|c|c|c|c|c|}
\hline Spitzer ID & Epoch ID & RA (J2000) & Dec (J2000) & Class & X-ray, Var., O? ${ }^{a}$ \\
\hline 188 & 3 & $8: 59: 32.11$ & $-47: 29: 24.95$ & II & $\ldots$ \\
\hline 189 & 2 & $8: 59: 19.80$ & $-47: 24: 01.38$ & II & $\mathrm{x}$ \\
\hline 191 & 4 & $8: 58: 20.41$ & $-47: 24: 07.82$ & II & $\cdots$ \\
\hline 192 & 4 & $8: 58: 48.39$ & $-47: 29: 29.73$ & II & $\cdots$ \\
\hline 196 & 4 & $8: 58: 46.69$ & $-47: 30: 35.73$ & II & $\mathrm{x}$ \\
\hline 197 & 3 & $8: 58: 55.56$ & $-47: 27: 45.20$ & II & $\mathrm{x}$ \\
\hline 199 & 3 & $8: 58: 22.00$ & $-47: 31: 07.54$ & II & $\ldots$ \\
\hline 200 & 3 & $8: 59: 02.46$ & $-47: 29: 47.76$ & II & $\mathrm{x}$ \\
\hline 202 & 3 & $8: 59: 30.01$ & $-47: 26: 33.87$ & II & $\mathrm{x}$ \\
\hline 204 & 1 & $8: 58: 00.70$ & $-47: 22: 02.64$ & II & $\cdots$ \\
\hline 205 & 3 & $8: 59: 35.54$ & $-47: 30: 48.08$ & II & $\ldots$ \\
\hline 206 & 2 & $8: 57: 42.25$ & $-47: 26: 04.60$ & II & $\cdots$ \\
\hline 209 & 3 & $8: 59: 26.26$ & $-47: 27: 54.60$ & II & $\mathrm{x}$ \\
\hline 211 & 3 & $8: 59: 27.84$ & $-47: 28: 03.04$ & II & $\mathrm{x}, \mathrm{v}$ \\
\hline 212 & 3 & $8: 59: 31.00$ & $-47: 30: 02.98$ & II & $\cdots$ \\
\hline 213 & 3 & $8: 59: 08.49$ & $-47: 31: 57.25$ & II & $\ldots$ \\
\hline 216 & 3 & $8: 58: 34.64$ & $-47: 28: 16.43$ & II & $\mathrm{x}$ \\
\hline 217 & 4 & $8: 58: 54.58$ & $-47: 24: 24.20$ & II & $\ldots$ \\
\hline 218 & 3 & $8: 59: 03.84$ & $-47: 24: 56.74$ & II & $\mathrm{x}$ \\
\hline 219 & 1 & $8: 57: 59.72$ & $-47: 22: 22.45$ & II & $\ldots$ \\
\hline 222 & 3 & $8: 58: 32.80$ & $-47: 25: 56.65$ & II & $\cdots$ \\
\hline 223 & 3 & $8: 58: 53.44$ & $-47: 30: 32.30$ & II & $\ldots$ \\
\hline 224 & 4 & $8: 57: 43.70$ & $-47: 25: 21.53$ & II & $\ldots$ \\
\hline 226 & 4 & $8: 57: 59.04$ & $-47: 25: 23.40$ & II & $\ldots$ \\
\hline 228 & 3 & $8: 59: 22.20$ & $-47: 32: 43.61$ & II & $\ldots$ \\
\hline 231 & 4 & $8: 58: 16.75$ & $-47: 24: 46.42$ & II & $\cdots$ \\
\hline 232 & 4 & $8: 58: 00.67$ & $-47: 25: 34.51$ & II & $\ldots$ \\
\hline 233 & 3 & $8: 58: 36.26$ & $-47: 25: 24.30$ & II & $\cdots$ \\
\hline 234 & 1 & $8: 58: 23.79$ & $-47: 23: 24.80$ & II & $\mathrm{v}$ \\
\hline 235 & 1 & $8: 58: 47.83$ & $-47: 30: 18.33$ & II & $\ldots$ \\
\hline 236 & 3 & $8: 59: 00.40$ & $-47: 25: 59.14$ & II & $\mathrm{x}$ \\
\hline 237 & 1 & $8: 58: 12.95$ & $-47: 22: 45.57$ & II & $\ldots$ \\
\hline 238 & 3 & $8: 59: 07.37$ & $-47: 31: 36.08$ & II & $\mathrm{x}$ \\
\hline 241 & 3 & $8: 59: 07.14$ & $-47: 28: 18.65$ & II & $\ldots$ \\
\hline 242 & 4 & $8: 58: 54.99$ & $-47: 28: 02.67$ & II & $\ldots$ \\
\hline 244 & 3 & $8: 59: 28.82$ & $-47: 32: 21.67$ & II & $\mathrm{x}, \mathrm{O}$ \\
\hline 245 & 1 & $8: 58: 03.48$ & $-47: 25: 02.53$ & II & $\cdots$ \\
\hline 246 & 3 & $8: 59: 35.38$ & $-47: 27: 23.39$ & II & $\mathrm{x}$ \\
\hline 247 & 3 & $8: 59: 11.24$ & $-47: 22: 37.58$ & II & $\mathrm{v}$ \\
\hline 248 & 4 & $8: 58: 05.29$ & $-47: 24: 32.31$ & II & $\mathrm{v}$ \\
\hline 249 & 3 & 8:59:01.95 & $-47: 31: 15.04$ & II & $\mathrm{x}$ \\
\hline 250 & 1 & 8:58:02.09 & $-47: 19: 43.08$ & II & $\cdots$ \\
\hline 251 & 3 & $8: 59: 03.35$ & $-47: 28: 53.60$ & II & $\mathrm{x}$ \\
\hline 252 & 3 & $8: 59: 10.04$ & $-47: 28: 24.12$ & II & $\mathrm{x}$ \\
\hline 253 & 4 & $8: 59: 28.73$ & $-47: 29: 24.77$ & II & $\ldots$ \\
\hline 254 & 3 & $8: 58: 47.98$ & $-47: 29: 40.98$ & II & $\ldots$ \\
\hline
\end{tabular}


Table 5-Continued

\begin{tabular}{|c|c|c|c|c|c|}
\hline Spitzer ID & Epoch ID & RA (J2000) & Dec (J2000) & Class & X-ray, Var., O? ${ }^{a}$ \\
\hline 256 & 3 & $8: 59: 10.40$ & $-47: 27: 40.11$ & II & $\ldots$ \\
\hline 257 & 3 & $8: 59: 14.46$ & $-47: 31: 18.94$ & II & $\mathrm{x}$ \\
\hline 259 & 3 & $8: 59: 14.45$ & $-47: 30: 50.63$ & II & $\mathrm{x}$ \\
\hline 261 & 2 & $8: 58: 00.57$ & $-47: 23: 04.87$ & II & $\ldots$ \\
\hline 264 & 3 & $8: 59: 10.57$ & $-47: 23: 49.43$ & II & $\mathrm{x}$ \\
\hline 265 & 3 & $8: 58: 38.22$ & $-47: 29: 27.89$ & II & $\ldots$ \\
\hline 267 & 1 & $8: 58: 05.58$ & $-47: 22: 10.20$ & II & $\cdots$ \\
\hline 268 & 3 & $8: 59: 24.90$ & $-47: 24: 50.70$ & II & $\mathrm{x}$ \\
\hline 269 & 4 & $8: 58: 51.03$ & $-47: 27: 43.03$ & II & $\ldots$ \\
\hline 272 & 4 & $8: 58: 16.69$ & $-47: 25: 33.66$ & II & $\mathrm{v}$ \\
\hline 273 & 3 & $8: 58: 48.92$ & $-47: 31: 31.87$ & II & $\mathrm{x}$ \\
\hline 275 & 1 & $8: 58: 17.44$ & $-47: 22: 03.55$ & II & $\ldots$ \\
\hline 276 & 4 & $8: 58: 16.31$ & $-47: 25: 03.93$ & II & $\cdots$ \\
\hline 277 & 1 & $8: 58: 10.17$ & $-47: 23: 50.20$ & II & $\ldots$ \\
\hline 279 & 2 & $8: 58: 30.00$ & $-47: 32: 10.82$ & II & $\cdots$ \\
\hline 282 & 3 & $8: 58: 55.25$ & $-47: 31: 54.94$ & II & $\mathrm{x}$ \\
\hline 283 & 4 & $8: 58: 26.48$ & $-47: 24: 16.43$ & II & $\ldots$ \\
\hline 284 & 1 & $8: 57: 58.83$ & $-47: 17: 09.88$ & II & $\ldots$ \\
\hline 285 & 3 & $8: 59: 15.68$ & $-47: 26: 08.90$ & II & $\mathrm{x}$ \\
\hline 286 & 1 & $8: 58: 24.50$ & $-47: 23: 37.40$ & II & $\cdots$ \\
\hline 287 & 3 & $8: 58: 44.64$ & $-47: 28: 42.15$ & II & $\ldots$ \\
\hline 288 & 1 & $8: 58: 10.39$ & $-47: 23: 08.87$ & II & $\ldots$ \\
\hline 289 & 1 & $8: 58: 25.86$ & $-47: 23: 04.86$ & II & $\ldots$ \\
\hline 293 & 3 & $8: 59: 26.70$ & $-47: 33: 10.14$ & II & $\mathrm{x}$ \\
\hline 294 & 1 & $8: 58: 17.20$ & $-47: 24: 27.25$ & II & $\ldots$ \\
\hline 296 & 1 & $8: 58: 14.87$ & $-47: 24: 57.61$ & II & $\ldots$ \\
\hline 298 & 1 & $8: 58: 20.09$ & $-47: 23: 52.97$ & II & $\mathrm{v}$ \\
\hline 299 & 4 & $8: 58: 08.45$ & $-47: 25: 35.97$ & II & $\ldots$ \\
\hline 300 & 4 & $8: 58: 09.65$ & $-47: 26: 41.01$ & II & $\cdots$ \\
\hline 301 & 1 & $8: 58: 01.51$ & $-47: 24: 47.00$ & II & $\ldots$ \\
\hline 303 & 3 & $8: 59: 17.25$ & $-47: 30: 11.07$ & II & $\mathrm{x}$ \\
\hline 304 & 1 & $8: 58: 04.40$ & $-47: 23: 05.62$ & II & $\ldots$ \\
\hline 305 & 4 & $8: 59: 30.58$ & $-47: 27: 59.76$ & II & $\mathrm{x}$ \\
\hline 307 & 3 & $8: 59: 07.82$ & $-47: 31: 36.99$ & II & $\mathrm{x}$ \\
\hline 308 & 3 & $8: 59: 19.98$ & $-47: 26: 19.33$ & II & $\mathrm{v}$ \\
\hline 309 & 4 & $8: 57: 59.50$ & $-47: 24: 44.77$ & II & . \\
\hline 310 & 3 & $8: 59: 28.92$ & $-47: 31: 40.50$ & II & $\mathrm{x}$ \\
\hline 311 & 3 & $8: 58: 37.82$ & $-47: 31: 17.27$ & II & $\cdots$ \\
\hline 312 & 3 & $8: 59: 23.61$ & $-47: 26: 49.95$ & II & $\mathrm{x}$ \\
\hline 313 & 3 & $8: 58: 38.16$ & $-47: 33: 13.73$ & II & $\cdots$ \\
\hline 315 & 3 & $8: 59: 28.57$ & $-47: 33: 35.81$ & II & $\mathrm{x}$ \\
\hline 319 & 1 & $8: 58: 09.04$ & $-47: 23: 20.55$ & II & $\cdots$ \\
\hline 321 & 1 & $8: 57: 58.60$ & $-47: 23: 55.83$ & II & $\ldots$ \\
\hline 322 & 3 & $8: 59: 02.07$ & $-47: 33: 41.00$ & II & $\cdots$ \\
\hline 323 & 3 & $8: 58: 30.71$ & $-47: 25: 45.35$ & II & $\ldots$ \\
\hline 326 & 1 & $8: 58: 38.04$ & $-47: 31: 09.23$ & II & $\cdots$ \\
\hline
\end{tabular}


Table 5-Continued

\begin{tabular}{|c|c|c|c|c|c|}
\hline Spitzer ID & Epoch ID & RA (J2000) & Dec (J2000) & Class & X-ray, Var., O? ${ }^{a}$ \\
\hline 328 & 3 & $8: 59: 18.32$ & $-47: 28: 30.97$ & II & $\mathrm{v}$ \\
\hline 329 & 4 & $8: 58: 09.22$ & $-47: 25: 45.52$ & II & $\cdots$ \\
\hline 330 & 3 & $8: 58: 48.96$ & $-47: 26: 45.57$ & II & $\ldots$ \\
\hline 331 & 4 & $8: 59: 03.69$ & $-47: 26: 36.68$ & II & $\mathrm{v}$ \\
\hline 332 & 3 & $8: 59: 00.10$ & $-47: 30: 06.88$ & II & $\mathrm{x}$ \\
\hline 333 & 3 & $8: 58: 58.85$ & $-47: 27: 24.96$ & II & $\mathrm{x}$ \\
\hline 335 & 3 & 8:59:09.02 & $-47: 21: 17.42$ & II & $\cdots$ \\
\hline 336 & 4 & $8: 58: 02.13$ & $-47: 24: 11.91$ & II & $\cdots$ \\
\hline 338 & 3 & $8: 58: 46.06$ & $-47: 29: 59.02$ & II & $\cdots$ \\
\hline 339 & 3 & $8: 59: 25.54$ & $-47: 29: 11.11$ & II & $\mathrm{x}$ \\
\hline 340 & 3 & $8: 59: 44.49$ & $-47: 33: 18.40$ & II & $\cdots$ \\
\hline 341 & 3 & $8: 58: 57.94$ & $-47: 30: 31.19$ & II & $\mathrm{x}$ \\
\hline 342 & 4 & $8: 57: 53.54$ & $-47: 24: 49.61$ & II & $\cdots$ \\
\hline 343 & 3 & $8: 59: 28.61$ & $-47: 29: 37.86$ & II & $\mathrm{x}, \mathrm{v}$ \\
\hline 347 & 3 & $8: 58: 53.94$ & $-47: 17: 18.12$ & II & $\cdots$ \\
\hline 348 & 1 & $8: 59: 40.95$ & $-47: 29: 38.36$ & II & $\ldots$ \\
\hline 349 & 1 & $8: 57: 55.88$ & $-47: 24: 12.15$ & II & $\ldots$ \\
\hline 350 & 3 & $8: 58: 44.65$ & $-47: 30: 34.55$ & II & $\ldots$ \\
\hline 355 & 3 & $8: 58: 51.63$ & $-47: 16: 22.52$ & II & $\mathrm{x}$ \\
\hline 357 & 4 & $8: 58: 08.25$ & $-47: 26: 43.22$ & II & $\cdots$ \\
\hline 359 & 4 & $8: 58: 14.17$ & $-47: 24: 39.71$ & II & $\ldots$ \\
\hline 360 & 3 & $8: 58: 25.40$ & $-47: 28: 17.98$ & II & $\cdots$ \\
\hline 361 & 3 & $8: 58: 50.39$ & $-47: 33: 19.53$ & II & $\mathrm{x}$ \\
\hline 363 & 3 & $8: 59: 13.59$ & $-47: 33: 08.73$ & II & $\ldots$ \\
\hline 364 & 4 & $8: 58: 10.51$ & $-47: 24: 56.86$ & II & $\cdots$ \\
\hline 365 & 3 & $8: 58: 46.29$ & $-47: 27: 51.40$ & II & $\mathrm{v}$ \\
\hline 367 & 3 & 8:59:09.62 & $-47: 28: 12.32$ & II & $\mathrm{x}$ \\
\hline 368 & 3 & $8: 59: 23.92$ & $-47: 28: 11.72$ & II & $\mathrm{x}$ \\
\hline 369 & 3 & $8: 59: 33.15$ & $-47: 33: 18.65$ & II & $\cdots$ \\
\hline 370 & 3 & $8: 58: 57.11$ & $-47: 32: 38.46$ & II & $\mathrm{x}$ \\
\hline 371 & 2 & 8:58:13.93 & $-47: 29: 15.05$ & II & $\cdots$ \\
\hline 374 & 3 & $8: 58: 49.50$ & $-47: 27: 21.82$ & II & $\ldots$ \\
\hline 376 & 3 & $8: 58: 51.89$ & $-47: 30: 33.16$ & II & $\cdots$ \\
\hline 377 & 1 & $8: 59: 51.55$ & $-47: 33: 09.12$ & II & $\mathrm{x}$ \\
\hline 378 & 3 & $8: 58: 54.97$ & $-47: 26: 58.63$ & II & $\mathrm{x}$ \\
\hline 379 & 3 & $8: 59: 16.31$ & $-47: 28: 41.65$ & II & $\mathrm{x}$ \\
\hline 383 & 3 & $8: 58: 41.04$ & $-47: 33: 08.72$ & II & $\ldots$ \\
\hline 384 & 3 & $8: 58: 58.30$ & $-47: 32: 57.87$ & II & $\mathrm{x}$ \\
\hline 385 & 3 & $8: 59: 12.75$ & $-47: 29: 59.90$ & II & $\mathrm{x}$ \\
\hline 386 & 4 & $8: 58: 15.68$ & $-47: 26: 43.02$ & II & $\cdots$ \\
\hline 387 & 1 & $8: 58: 06.45$ & $-47: 21: 57.70$ & II & $\mathrm{v}$ \\
\hline 389 & 1 & $8: 58: 01.73$ & $-47: 22: 22.26$ & II & $\cdots$ \\
\hline 390 & 3 & $8: 58: 51.99$ & $-47: 32: 46.00$ & II & $\mathrm{v}$ \\
\hline 391 & 3 & $8: 59: 23.10$ & $-47: 27: 51.38$ & II & $\cdots$ \\
\hline 392 & 3 & $8: 59: 15.48$ & $-47: 32: 16.84$ & II & $\ldots$ \\
\hline 393 & 4 & $8: 57: 51.30$ & $-47: 28: 24.61$ & II & $\cdots$ \\
\hline
\end{tabular}


Table 5-Continued

\begin{tabular}{|c|c|c|c|c|c|}
\hline Spitzer ID & Epoch ID & RA (J2000) & Dec (J2000) & Class & X-ray, Var., O? ${ }^{a}$ \\
\hline 394 & 3 & $8: 59: 01.56$ & $-47: 31: 10.11$ & II & $\mathrm{x}$ \\
\hline 395 & 1 & $8: 58: 16.33$ & $-47: 24: 43.43$ & II & $\ldots$ \\
\hline 397 & 3 & $8: 59: 36.77$ & $-47: 29: 21.84$ & II & $\mathrm{x}$ \\
\hline 399 & 3 & $8: 58: 54.26$ & $-47: 29: 49.77$ & II & $\mathrm{x}$ \\
\hline 401 & 1 & $8: 58: 20.99$ & $-47: 25: 15.16$ & II & $\ldots$ \\
\hline 402 & 3 & $8: 58: 59.54$ & $-47: 29: 52.30$ & II & $\mathrm{x}$ \\
\hline 405 & 1 & $8: 57: 49.69$ & $-47: 23: 58.96$ & II & $\ldots$ \\
\hline 406 & 4 & $8: 59: 26.52$ & $-47: 29: 18.32$ & II & $\ldots$ \\
\hline 407 & 3 & $8: 58: 49.96$ & $-47: 28: 57.46$ & II & $\mathrm{x}$ \\
\hline 408 & 3 & $8: 59: 33.32$ & $-47: 32: 53.67$ & II & $\mathrm{x}$ \\
\hline 409 & 3 & $8: 59: 43.77$ & $-47: 29: 36.09$ & II & $\cdots$ \\
\hline 410 & 4 & $8: 58: 16.97$ & $-47: 24: 06.78$ & II & $\ldots$ \\
\hline 411 & 3 & $8: 58: 49.29$ & $-47: 27: 08.41$ & II & $\cdots$ \\
\hline 412 & 3 & $8: 58: 54.03$ & $-47: 31: 2.22$ & II & $\mathrm{x}$ \\
\hline 413 & 3 & $8: 58: 36.16$ & $-47: 31: 35.76$ & II & $\mathrm{x}$ \\
\hline 415 & 3 & $8: 59: 36.94$ & $-47: 33: 04.65$ & II & $\mathrm{x}$ \\
\hline 416 & 3 & $8: 58: 42.58$ & $-47: 31: 56.69$ & II & $\mathrm{x}$ \\
\hline 417 & 3 & $8: 59: 15.85$ & $-47: 28: 52.90$ & II & $\mathrm{x}$ \\
\hline 418 & 3 & $8: 59: 15.38$ & $-47: 27: 46.24$ & II & $\cdots$ \\
\hline 419 & 2 & $8: 59: 41.70$ & $-47: 30: 04.18$ & II & $\ldots$ \\
\hline 420 & 1 & 9:00:05.64 & $-47: 27: 12.09$ & II & $\ldots$ \\
\hline 421 & 1 & $9: 00: 10.47$ & $-47: 27: 05.35$ & II & $\ldots$ \\
\hline 422 & 1 & 9:00:22.98 & $-47: 18: 57.91$ & II & $\ldots$ \\
\hline 423 & 3 & $8: 59: 48.35$ & $-47: 26: 21.29$ & II & $\mathrm{x}$ \\
\hline 424 & 3 & $8: 59: 21.10$ & $-47: 22: 28.39$ & II & $\ldots$ \\
\hline 425 & 1 & $8: 59: 46.72$ & $-47: 17: 26.70$ & II & $\ldots$ \\
\hline 426 & 1 & $8: 59: 38.80$ & $-47: 16: 42.72$ & II & $\ldots$ \\
\hline 428 & 1 & $8: 59: 48.12$ & $-47: 17: 24.99$ & II & $\cdots$ \\
\hline 429 & 1 & $8: 59: 45.04$ & $-47: 17: 11.19$ & II & $\ldots$ \\
\hline 430 & 3 & $8: 59: 53.63$ & $-47: 28: 45.99$ & II & $\mathrm{x}$ \\
\hline 431 & 3 & $8: 59: 29.14$ & $-47: 23: 42.33$ & II & $\cdots$ \\
\hline 432 & 3 & $8: 59: 57.36$ & $-47: 27: 29.22$ & II & $\mathrm{x}$ \\
\hline 433 & 3 & 9:00:07.92 & $-47: 23: 30.95$ & II & $\ldots$ \\
\hline 435 & 3 & $8: 59: 33.85$ & $-47: 19: 49.83$ & II & $\ldots$ \\
\hline 436 & 1 & $8: 59: 52.55$ & $-47: 24: 13.12$ & II & $\ldots$ \\
\hline 441 & 3 & $8: 59: 43.81$ & $-47: 22: 33.63$ & II & $\ldots$ \\
\hline 442 & 1 & 9:00:10.93 & $-47: 32: 03.65$ & II & $\ldots$ \\
\hline 444 & 3 & $8: 59: 39.00$ & $-47: 27: 20.13$ & II & $\mathrm{x}$ \\
\hline 445 & 3 & $8: 59: 30.11$ & $-47: 23: 00.51$ & II & $\ldots$ \\
\hline 446 & 1 & $8: 59: 25.16$ & $-47: 15: 52.72$ & II & $\ldots$ \\
\hline 448 & 1 & $8: 59: 49.83$ & $-47: 18: 00.13$ & II & $\ldots$ \\
\hline 449 & 1 & 9:00:06.68 & $-47: 30: 32.44$ & II & $\ldots$ \\
\hline 450 & 3 & $8: 59: 44.56$ & $-47: 18: 06.49$ & II & $\ldots$ \\
\hline 451 & 4 & $8: 59: 57.92$ & $-47: 32: 14.70$ & II & $\mathrm{x}$ \\
\hline 452 & 1 & $8: 59: 45.87$ & $-47: 17: 31.50$ & II & $\ldots$ \\
\hline 456 & 3 & $8: 59: 52.85$ & $-47: 19: 33.65$ & II & $\ldots$ \\
\hline
\end{tabular}


Table 5-Continued

\begin{tabular}{|c|c|c|c|c|c|}
\hline Spitzer ID & Epoch ID & RA (J2000) & Dec (J2000) & Class & X-ray, Var., O? ${ }^{a}$ \\
\hline 457 & 1 & $8: 59: 43.58$ & $-47: 17: 33.77$ & II & $\ldots$ \\
\hline 461 & 1 & $8: 59: 11.92$ & $-47: 14: 41.83$ & II & $\cdots$ \\
\hline 462 & 3 & $8: 59: 39.64$ & $-47: 17: 44.15$ & II & $\ldots$ \\
\hline 464 & 2 & 9:00:05.33 & $-47: 23: 54.42$ & II & $\ldots$ \\
\hline 465 & 3 & $8: 59: 50.41$ & $-47: 26: 56.75$ & II & $\mathrm{x}$ \\
\hline 469 & 1 & $8: 59: 12.15$ & $-47: 16: 19.46$ & II & $\ldots$ \\
\hline 470 & 2 & $8: 58: 21.65$ & $-47: 39: 20.34$ & II & $\cdots$ \\
\hline 473 & 3 & 8:59:09.70 & $-47: 24: 38.50$ & II & $\mathrm{x}$ \\
\hline 474 & 2 & $8: 59: 09.03$ & $-47: 25: 37.57$ & II & $\ldots$ \\
\hline 476 & 3 & $8: 59: 07.48$ & $-47: 29: 15.84$ & II & $\mathrm{x}$ \\
\hline 479 & 3 & $8: 59: 15.71$ & $-47: 29: 39.34$ & II & $\mathrm{x}$ \\
\hline 480 & 2 & $8: 58: 56.78$ & $-47: 39: 38.50$ & II & $\ldots$ \\
\hline 483 & 3 & $8: 59: 21.26$ & $-47: 33: 24.56$ & II & $\mathrm{x}$ \\
\hline 485 & 3 & $8: 59: 32.50$ & $-47: 38: 55.68$ & II & $\ldots$ \\
\hline 486 & 1 & 9:00:06.44 & $-47: 29: 31.55$ & II & $\mathrm{v}$ \\
\hline 487 & 1 & $9: 00: 26.76$ & $-47: 26: 57.52$ & II & $\ldots$ \\
\hline 488 & 3 & $8: 58: 33.45$ & $-47: 24: 58.24$ & II & $\ldots$ \\
\hline 489 & 3 & $8: 58: 49.81$ & $-47: 29: 24.84$ & II & $\mathrm{x}$ \\
\hline 491 & 3 & $8: 58: 51.80$ & $-47: 31: 27.20$ & II & $\mathrm{x}$ \\
\hline 494 & 4 & $8: 59: 16.01$ & $-47: 31: 10.23$ & II & $\mathrm{x}$ \\
\hline 495 & 2 & 9:00:39.50 & $-47: 41: 10.43$ & II & $\ldots$ \\
\hline 496 & 2 & $8: 58: 25.43$ & $-47: 43: 23.64$ & II & $\ldots$ \\
\hline 497 & 2 & $8: 58: 25.93$ & $-47: 41: 19.37$ & II & $\ldots$ \\
\hline 498 & 2 & $8: 58: 26.87$ & $-47: 40: 52.40$ & II & $\ldots$ \\
\hline 499 & 2 & 9:00:38.07 & $-47: 40: 45.98$ & II & $\ldots$ \\
\hline 500 & 3 & $8: 58: 59.89$ & $-47: 35: 52.23$ & II & $\mathrm{x}$ \\
\hline 501 & 3 & $8: 58: 49.35$ & $-47: 34: 05.94$ & II & $\ldots$ \\
\hline 503 & 2 & $8: 58: 04.03$ & $-47: 39: 24.31$ & II & $\ldots$ \\
\hline 504 & 2 & $8: 58: 40.46$ & $-47: 40: 47.51$ & II & $\cdots$ \\
\hline 506 & 2 & $8: 58: 34.75$ & $-47: 40: 58.30$ & II & $\ldots$ \\
\hline 507 & 2 & $8: 58: 13.99$ & $-47: 40: 12.60$ & II & $\cdots$ \\
\hline 508 & 2 & $8: 57: 57.43$ & $-47: 40: 55.87$ & II & $\ldots$ \\
\hline 509 & 2 & $8: 58: 32.09$ & $-47: 41: 16.59$ & II & $\cdots$ \\
\hline 510 & 2 & $8: 58: 24.28$ & $-47: 39: 46.60$ & II & $\ldots$ \\
\hline 512 & 3 & $8: 58: 49.83$ & $-47: 35: 20.62$ & II & $\mathrm{x}$ \\
\hline 513 & 2 & $8: 58: 26.11$ & $-47: 41: 56.25$ & II & $\ldots$ \\
\hline 514 & 3 & $8: 58: 55.11$ & $-47: 35: 00.09$ & II & $\mathrm{x}$ \\
\hline 515 & 2 & $8: 58: 40.97$ & $-47: 46: 40.16$ & II & $\cdots$ \\
\hline 518 & 3 & $8: 58: 44.53$ & $-47: 34: 06.49$ & II & $\mathrm{x}$ \\
\hline 519 & 2 & $8: 58: 00.56$ & $-47: 40: 42.08$ & II & $\cdots$ \\
\hline 520 & 2 & $8: 58: 01.93$ & $-47: 39: 44.11$ & II & $\ldots$ \\
\hline 521 & 2 & $8: 58: 04.20$ & $-47: 39: 45.34$ & II & $\cdots$ \\
\hline 522 & 2 & $8: 58: 13.78$ & $-47: 40: 53.96$ & II & $\ldots$ \\
\hline 523 & 3 & $8: 58: 44.19$ & $-47: 34: 41.36$ & II & $\cdots$ \\
\hline 525 & 2 & $8: 59: 08.58$ & $-47: 44: 06.66$ & II & $\ldots$ \\
\hline 526 & 2 & $8: 58: 02.22$ & $-47: 39: 40.56$ & II & $\cdots$ \\
\hline
\end{tabular}


Table 5-Continued

\begin{tabular}{|c|c|c|c|c|c|}
\hline Spitzer ID & Epoch ID & RA (J2000) & Dec (J2000) & Class & X-ray, Var., O? ${ }^{\text {a }}$ \\
\hline 527 & 2 & $8: 58: 25.86$ & $-47: 42: 58.19$ & II & $\cdots$ \\
\hline 528 & 2 & $8: 59: 14.84$ & $-47: 44: 02.60$ & II & $\cdots$ \\
\hline 529 & 2 & $8: 58: 29.90$ & $-47: 41: 34.34$ & II & $\cdots$ \\
\hline 530 & 3 & $8: 58: 37.53$ & $-47: 34: 41.13$ & II & $\cdots$ \\
\hline 532 & 2 & $8: 58: 25.98$ & $-47: 44: 09.87$ & II & $\cdots$ \\
\hline 533 & 2 & 8:58:03.67 & $-47: 40: 28.32$ & II & $\cdots$ \\
\hline 534 & 2 & 8:58:05.06 & $-47: 40: 27.97$ & II & $\cdots$ \\
\hline 535 & 2 & $8: 58: 53.86$ & $-47: 45: 10.09$ & II & $\cdots$ \\
\hline 537 & 2 & 8:58:08.49 & $-47: 40: 35.53$ & II & $\cdots$ \\
\hline 538 & 2 & 8:58:53.42 & $-47: 42: 41.74$ & II & $\ldots$ \\
\hline 539 & 2 & $8: 58: 30.31$ & $-47: 41: 18.74$ & II & $\cdots$ \\
\hline 540 & 3 & $8: 58: 54.85$ & $-47: 34: 33.30$ & II & $\mathrm{x}$ \\
\hline 541 & 2 & $8: 59: 28.06$ & $-47: 44: 45.87$ & II & $\cdots$ \\
\hline 542 & 2 & $8: 58: 21.08$ & $-47: 40: 45.16$ & II & $\ldots$ \\
\hline 544 & 2 & 9:00:0.77 & $-47: 43: 51.23$ & II & $\cdots$ \\
\hline 545 & 2 & $8: 58: 21.45$ & $-47: 40: 08.28$ & II & $\ldots$ \\
\hline 546 & 2 & 9:00:5.39 & $-47: 39: 44.85$ & II & $\cdots$ \\
\hline 547 & 2 & $8: 59: 59.17$ & $-47: 44: 21.82$ & II & $\ldots$ \\
\hline 548 & 2 & $9: 00: 22.16$ & $-47: 41: 11.51$ & II & $\cdots$ \\
\hline 549 & 2 & 9:00:12.58 & $-47: 39: 54.94$ & II & $\cdots$ \\
\hline 550 & 2 & $8: 58: 59.08$ & $-47: 28: 13.56$ & II & $\ldots$ \\
\hline 551 & 3 & 8:59:07.31 & $-47: 23: 35.86$ & II & $\mathrm{x}$ \\
\hline 552 & 3 & $8: 58: 30.77$ & $-47: 33: 57.46$ & II & $\cdots$ \\
\hline 556 & 4 & 8:58:29.63 & $-47: 33: 45.93$ & II & $\cdots$ \\
\hline 557 & 2 & 8:58:05.91 & $-47: 21: 52.29$ & II & $\ldots$ \\
\hline 558 & 4 & $8: 58: 59.83$ & $-47: 26: 23.51$ & II & $\ldots$ \\
\hline 560 & 3 & 8:59:00.29 & $-47: 29: 40.47$ & II & $\mathrm{x}$ \\
\hline 563 & 3 & $8: 58: 19.82$ & $-47: 32: 30.38$ & II & $\cdots$ \\
\hline 564 & 3 & $8: 58: 47.54$ & $-47: 33: 22.88$ & II & $\mathrm{x}$ \\
\hline 565 & 4 & $8: 59: 07.97$ & $-47: 31: 0.07$ & II & $\mathrm{x}$ \\
\hline 566 & 2 & 9:00:15.58 & $-47: 43: 35.61$ & II & $\cdots$ \\
\hline 567 & 2 & 9:00:36.40 & $-47: 42: 43.23$ & II & $\cdots$ \\
\hline 568 & 2 & $8: 58: 21.96$ & $-47: 41: 14.24$ & II & $\cdots$ \\
\hline 569 & 2 & $8: 58: 19.97$ & $-47: 39: 40.25$ & II & $\cdots$ \\
\hline 570 & 3 & $8: 58: 43.28$ & $-47: 34: 54.72$ & II & $\mathrm{x}$ \\
\hline 574 & 3 & $8: 58: 25.16$ & $-47: 34: 44.35$ & II & $\cdots$ \\
\hline 575 & 3 & $8: 59: 07.63$ & $-47: 35: 20.06$ & II & $\mathrm{v}$ \\
\hline 579 & 3 & $8: 58: 49.59$ & $-47: 32: 55.38$ & II & $\mathrm{x}$ \\
\hline 582 & 3 & $8: 58: 52.79$ & $-47: 33: 15.10$ & II & $\mathrm{x}$ \\
\hline 583 & 3 & $8: 58: 52.87$ & $-47: 27: 39.48$ & II & $\cdots$ \\
\hline 584 & 3 & $8: 59: 09.46$ & $-47: 30: 20.64$ & II & $\mathrm{x}$ \\
\hline 587 & 3 & $8: 59: 05.65$ & $-47: 30: 40.94$ & II & $\mathrm{x}$ \\
\hline 608 & 2 & $8: 59: 28.11$ & $-47: 39: 32.35$ & II & o \\
\hline 625 & 2 & $8: 58: 31.84$ & $-47: 39: 39.07$ & II & $\cdots$ \\
\hline 641 & 1 & $8: 57: 47.99$ & $-47: 27: 59.95$ & II & $\ldots$ \\
\hline 651 & 4 & $8: 58: 30.94$ & $-47: 32: 19.59$ & II & $\cdots$ \\
\hline
\end{tabular}


Table 5-Continued

\begin{tabular}{|c|c|c|c|c|c|}
\hline Spitzer ID & Epoch ID & RA (J2000) & Dec (J2000) & Class & X-ray, Var., O? ${ }^{a}$ \\
\hline 662 & 3 & $8: 59: 16.56$ & $-47: 27: 49.53$ & II & $\ldots$ \\
\hline 666 & 4 & $8: 58: 29.23$ & $-47: 25: 48.43$ & II & $\ldots$ \\
\hline 670 & 1 & $8: 57: 57.00$ & $-47: 29: 39.28$ & II & $\ldots$ \\
\hline 672 & 4 & $8: 58: 45.62$ & $-47: 25: 52.45$ & II & $\cdots$ \\
\hline 673 & 4 & $8: 59: 11.88$ & $-47: 27: 37.80$ & II & $\cdots$ \\
\hline 675 & 1 & $8: 58: 06.92$ & $-47: 28: 57.71$ & II & $\ldots$ \\
\hline 693 & 4 & $8: 58: 32.33$ & $-47: 32: 31.98$ & II & $\cdots$ \\
\hline 710 & 4 & $8: 58: 33.05$ & $-47: 32: 23.88$ & II & $\ldots$ \\
\hline 757 & 1 & $8: 58: 03.49$ & $-47: 26: 41.80$ & II & $\cdots$ \\
\hline 779 & 4 & $8: 59: 14.99$ & $-47: 36: 36.88$ & II & $\ldots$ \\
\hline 796 & 4 & $8: 58: 36.43$ & $-47: 33: 59.08$ & II & $\cdots$ \\
\hline 797 & 4 & $8: 58: 34.35$ & $-47: 33: 46.55$ & II & $\ldots$ \\
\hline 798 & 4 & $8: 59: 02.66$ & $-47: 31: 13.46$ & II & $\cdots$ \\
\hline 803 & 2 & $8: 58: 42.91$ & $-47: 39: 37.62$ & II & $\ldots$ \\
\hline 804 & 1 & $8: 57: 34.06$ & $-47: 27: 41.42$ & II & $\mathrm{o}, \mathrm{v}$ \\
\hline 805 & 4 & $8: 59: 19.50$ & $-47: 26: 01.35$ & II & $\ldots$ \\
\hline 806 & 1 & $8: 57: 41.31$ & $-47: 25: 32.67$ & II & o \\
\hline 807 & 1 & $8: 59: 45.02$ & $-47: 17: 20.85$ & II & $\ldots$ \\
\hline 808 & 2 & $8: 59: 31.31$ & $-47: 20: 38.75$ & II & o \\
\hline 809 & 1 & $8: 58: 57.14$ & $-47: 15: 05.33$ & II & o \\
\hline 810 & 4 & $8: 59: 31.39$ & $-47: 26: 04.60$ & II & $\ldots$ \\
\hline 811 & 4 & $8: 58: 24.23$ & $-47: 37: 13.07$ & II & o \\
\hline 812 & 4 & $8: 58: 37.31$ & $-47: 34: 27.60$ & II & . \\
\hline
\end{tabular}

${ }^{\mathrm{a}} \mathrm{x}$ : indicates Chandra X-ray detection. v: indicates source is a candidate variable. $\mathrm{o}$ : indicates source is an O-star candidate. 
Table 6. Astrometry of the Class III YSOs in RCW 38.

\begin{tabular}{|c|c|c|c|c|c|}
\hline Spitzer ID & Epoch ID & RA (J2000) & Dec (J2000) & Class & X-ray/Var ${ }^{a}$ \\
\hline 17 & 3 & $8: 59: 37.41$ & $-47: 34: 35.17$ & III & $\mathrm{x}$ \\
\hline 36 & 3 & 8:59:09.13 & $-47: 34: 05.24$ & III & $\mathrm{x}$ \\
\hline 65 & 3 & $8: 59: 25.72$ & $-47: 34: 36.20$ & III & $\mathrm{x}$ \\
\hline 77 & 3 & $8: 59: 27.72$ & $-47: 35: 50.73$ & III & $\mathrm{x}$ \\
\hline 82 & 3 & $8: 59: 08.84$ & $-47: 36: 37.77$ & III & $\mathrm{x}$ \\
\hline 93 & 1 & $8: 58: 48.81$ & $-47: 32: 15.96$ & III & $\mathrm{x}$ \\
\hline 99 & 3 & $8: 58: 43.51$ & $-47: 29: 34.32$ & III & $\mathrm{x}$ \\
\hline 100 & 1 & $8: 59: 46.88$ & $-47: 38: 04.42$ & III & $\mathrm{x}$ \\
\hline 109 & 3 & $8: 58: 42.68$ & $-47: 30: 15.48$ & III & $\mathrm{x}$ \\
\hline 123 & 3 & $8: 58: 55.85$ & $-47: 32: 51.22$ & III & $\mathrm{x}$ \\
\hline 125 & 3 & $8: 58: 49.14$ & $-47: 29: 17.21$ & III & $\mathrm{x}$ \\
\hline 129 & 4 & 8:59:56.09 & $-47: 33: 04.34$ & III & $\mathrm{x}$ \\
\hline 133 & 3 & $8: 59: 29.96$ & $-47: 31: 49.90$ & III & $\mathrm{x}$ \\
\hline 166 & 3 & $8: 58: 57.96$ & $-47: 27: 33.73$ & III & $\mathrm{x}$ \\
\hline 169 & 3 & $8: 59: 34.46$ & $-47: 27: 15.66$ & III & $\mathrm{x}$ \\
\hline 190 & 3 & $8: 58: 52.94$ & $-47: 33: 09.13$ & III & $\mathrm{x}$ \\
\hline 194 & 3 & $8: 59: 06.15$ & $-47: 30: 35.45$ & III & $\mathrm{x}$ \\
\hline 195 & 3 & $8: 58: 41.58$ & $-47: 28: 34.95$ & III & $\mathrm{x}$ \\
\hline 210 & 3 & $8: 59: 22.22$ & $-47: 25: 50.12$ & III & $\mathrm{x}$ \\
\hline 221 & 3 & $8: 58: 53.41$ & $-47: 26: 14.30$ & III & $\mathrm{x}$ \\
\hline 225 & 3 & $8: 58: 53.98$ & $-47: 26: 20.09$ & III & $\mathrm{x}$ \\
\hline 229 & 3 & $8: 59: 17.58$ & $-47: 27: 48.08$ & III & $\mathrm{x}$ \\
\hline 243 & 3 & $8: 59: 06.38$ & $-47: 29: 09.87$ & III & $\mathrm{x}$ \\
\hline 255 & 3 & $8: 59: 21.58$ & $-47: 29: 28.91$ & III & $\mathrm{x}$ \\
\hline 262 & 1 & $8: 59: 25.07$ & $-47: 27: 26.26$ & III & $\mathrm{x}$ \\
\hline 263 & 3 & $8: 59: 26.55$ & $-47: 30: 07.91$ & III & $\mathrm{x}$ \\
\hline 278 & 3 & $8: 59: 18.60$ & $-47: 25: 56.28$ & III & $\mathrm{x}$ \\
\hline 280 & 3 & $8: 59: 20.95$ & $-47: 29: 14.06$ & III & $\mathrm{x}$ \\
\hline 281 & 3 & $8: 59: 02.17$ & $-47: 30: 16.78$ & III & $\mathrm{x}$ \\
\hline 290 & 3 & $8: 59: 14.74$ & $-47: 29: 31.65$ & III & $\mathrm{x}$ \\
\hline 295 & 3 & $8: 59: 21.31$ & $-47: 25: 55.60$ & III & $\mathrm{x}$ \\
\hline 297 & 3 & $8: 59: 20.70$ & $-47: 26: 17.28$ & III & $\mathrm{x}$ \\
\hline 302 & 3 & $8: 59: 32.45$ & $-47: 32: 35.82$ & III & $\mathrm{x}$ \\
\hline 314 & 3 & $8: 58: 31.46$ & $-47: 28: 07.92$ & III & $\mathrm{x}$ \\
\hline 317 & 3 & 8:59:01.70 & $-47: 30: 37.19$ & III & $\mathrm{x}$ \\
\hline 318 & 4 & $8: 58: 05.67$ & $-47: 28: 00.17$ & III & $\mathrm{x}$ \\
\hline 320 & 3 & $8: 59: 22.86$ & $-47: 26: 33.61$ & III & $\mathrm{x}$ \\
\hline 327 & 3 & $8: 58: 50.66$ & $-47: 32: 39.65$ & III & $\mathrm{x}$ \\
\hline 334 & 3 & $8: 58: 44.40$ & $-47: 32: 53.30$ & III & $\mathrm{x}$ \\
\hline 337 & 3 & $8: 59: 05.48$ & $-47: 30: 02.64$ & III & $\mathrm{x}$ \\
\hline 345 & 3 & $8: 59: 49.64$ & $-47: 31: 16.80$ & III & $\mathrm{x}$ \\
\hline 354 & 4 & $8: 59: 00.27$ & $-47: 27: 58.42$ & III & $\mathrm{x}$ \\
\hline 358 & 3 & $8: 58: 57.79$ & $-47: 23: 58.87$ & III & $\mathrm{x}$ \\
\hline 366 & 3 & 8:59:09.17 & $-47: 28: 01.46$ & III & $\mathrm{x}$ \\
\hline 372 & 3 & $8: 59: 35.58$ & $-47: 31: 37.17$ & III & $\mathrm{x}$ \\
\hline 373 & 3 & $8: 59: 13.46$ & $-47: 32: 47.28$ & III & $\mathrm{x}$ \\
\hline
\end{tabular}


Table 6-Continued

\begin{tabular}{|c|c|c|c|c|c|}
\hline Spitzer ID & Epoch ID & RA (J2000) & $\operatorname{Dec}(\mathrm{J} 2000)$ & Class & $\mathrm{X}$-ray/Var ${ }^{2}$ \\
\hline 375 & 3 & $8: 58: 54.39$ & $-47: 25: 51.61$ & III & $\mathrm{x}$ \\
\hline 380 & 4 & $8: 59: 51.38$ & $-47: 33: 23.34$ & III & $\mathrm{x}$ \\
\hline 398 & 3 & $8: 58: 46.89$ & $-47: 33: 14.50$ & III & $\mathrm{x}$ \\
\hline 400 & 3 & $8: 59: 07.37$ & $-47: 33: 05.34$ & III & $\mathrm{x}$ \\
\hline 427 & 3 & $8: 59: 57.89$ & $-47: 27: 01.80$ & III & $\mathrm{x}$ \\
\hline 437 & 4 & 9:00:04.38 & $-47: 29: 46.85$ & III & $\mathrm{x}$ \\
\hline 439 & 4 & $8: 59: 58.00$ & $-47: 27: 07.93$ & III & $\mathrm{x}$ \\
\hline 440 & 3 & $8: 59: 48.36$ & $-47: 26: 40.76$ & III & $\mathrm{x}$ \\
\hline 447 & 3 & $8: 59: 30.67$ & $-47: 24: 49.68$ & III & $\mathrm{x}$ \\
\hline 454 & 3 & $8: 59: 45.68$ & $-47: 28: 41.76$ & III & $\mathrm{x}$ \\
\hline 455 & 3 & $8: 59: 40.89$ & $-47: 25: 26.49$ & III & $\mathrm{x}$ \\
\hline 458 & 3 & $8: 59: 53.89$ & $-47: 27: 42.60$ & III & $\mathrm{x}$ \\
\hline 463 & 4 & 9:00:04.84 & $-47: 27: 08.23$ & III & $\mathrm{x}$ \\
\hline 468 & 3 & $8: 58: 52.25$ & $-47: 24: 55.76$ & III & $\mathrm{x}$ \\
\hline 482 & 3 & $8: 59: 24.37$ & $-47: 31: 11.52$ & III & $\mathrm{x}$ \\
\hline 484 & 3 & $8: 59: 28.21$ & $-47: 35: 45.34$ & III & $\mathrm{x}$ \\
\hline 511 & 3 & $8: 58: 51.15$ & $-47: 34: 37.35$ & III & $\mathrm{x}$ \\
\hline 517 & 3 & $8: 58: 56.09$ & $-47: 35: 20.88$ & III & $\mathrm{x}$ \\
\hline 553 & 3 & $8: 59: 20.23$ & $-47: 28: 29.47$ & III & $\mathrm{x}$ \\
\hline 554 & 2 & $8: 59: 14.90$ & $-47: 29: 27.85$ & III & $\mathrm{x}$ \\
\hline 555 & 3 & $8: 58: 58.57$ & $-47: 30: 17.33$ & III & $\mathrm{x}$ \\
\hline 562 & 4 & $8: 59: 23.67$ & $-47: 25: 23.30$ & III & $\mathrm{x}$ \\
\hline 576 & 3 & $8: 59: 03.76$ & $-47: 27: 59.55$ & III & $\mathrm{x}$ \\
\hline 578 & 3 & $8: 58: 57.41$ & $-47: 27: 30.13$ & III & $\mathrm{x}$ \\
\hline 580 & 3 & $8: 59: 16.06$ & $-47: 33: 13.10$ & III & $\mathrm{x}$ \\
\hline 586 & 3 & $8: 59: 03.90$ & $-47: 31: 04.68$ & III & $\mathrm{x}$ \\
\hline 588 & 3 & $8: 58: 50.98$ & $-47: 29: 24.03$ & III & $\mathrm{x}$ \\
\hline 591 & 4 & $8: 59: 35.37$ & $-47: 28: 18.17$ & III & $\mathrm{x}$ \\
\hline
\end{tabular}

${ }^{\mathrm{a}} \mathrm{x}$ : indicates source is a Chandra X-ray detection. 
Table 7. Photometry of the Class 0/I YSOs in RCW 38.

\begin{tabular}{|c|c|c|c|c|c|c|c|c|c|c|}
\hline $\begin{array}{l}S p . \\
\text { ID }\end{array}$ & $\begin{array}{l}\text { Ep. } \\
\text { ID }\end{array}$ & $\begin{array}{c}\mathrm{J} \\
{[\mathrm{mag} .]}\end{array}$ & $\begin{array}{c}\mathrm{H} \\
{[\mathrm{mag} .]}\end{array}$ & $\begin{array}{c}\mathrm{K} \\
{[\mathrm{mag} .]}\end{array}$ & $\begin{array}{l}3.6 \mu \mathrm{m} \\
\text { [mag.] }\end{array}$ & $\begin{array}{l}4.5 \mu \mathrm{m} \\
\text { [mag.] }\end{array}$ & $\begin{array}{l}5.8 \mu \mathrm{m} \\
\text { [mag.] }\end{array}$ & $\begin{array}{l}8.0 \mu \mathrm{m} \\
{[\mathrm{mag} .]}\end{array}$ & $\alpha_{I R A C}$ & $\begin{array}{c}A_{K} \\
{[\mathrm{mag} .]}\end{array}$ \\
\hline 72 & 3 & $16.17 \pm 0.10$ & $15.03 \pm 0.07$ & $14.40 \pm 0.09$ & $13.08 \pm 0.14$ & $12.88 \pm 0.14$ & $10.85 \pm 0.23$ & 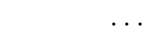 & 1.34 & 0.41 \\
\hline 114 & 3 & $\ldots$ & .. & $13.95 \pm 0.07$ & $11.35 \pm 0.10$ & $10.65 \pm 0.06$ & $9.28 \pm 0.18$ & . & 1.13 & $\ldots$ \\
\hline 138 & 3 & $\ldots$ & $\ldots$ & $15.00 \pm 0.16$ & $11.59 \pm 0.14$ & $10.68 \pm 0.13$ & . & $\ldots$ & 0.71 & $\ldots$ \\
\hline 141 & 3 & $14.66 \pm 0.05$ & $13.10 \pm 0.03$ & $\cdots$ & $9.88 \pm 0.02$ & $9.07 \pm 0.02$ & $8.16 \pm 0.02$ & $6.98 \pm 0.05$ & 0.49 & $\ldots$ \\
\hline 165 & 3 & $\cdots$ & $\cdots$ & $14.93 \pm 0.12$ & $12.33 \pm 0.07$ & $10.94 \pm 0.04$ & $9.96 \pm 0.05$ & $8.72 \pm 0.08$ & 1.23 & $\ldots$ \\
\hline 185 & 3 & $\ldots$ & $\ldots$ & $14.62 \pm 0.13$ & $12.58 \pm 0.10$ & $11.41 \pm 0.06$ & $9.88 \pm 0.13$ & $8.48 \pm 0.18$ & 1.95 & $\ldots$ \\
\hline 239 & 3 & $16.18 \pm 0.11$ & $14.83 \pm 0.08$ & $13.84 \pm 0.09$ & $13.18 \pm 0.21$ & $12.03 \pm 0.21$ & $\cdots$ & $\cdots$ & 1.65 & $\ldots$ \\
\hline 258 & 3 & $\ldots$ & $\ldots$ & $12.61 \pm 0.07$ & $10.29 \pm 0.09$ & $9.65 \pm 0.07$ & $\ldots$ & $\ldots$ & -0.35 & $\ldots$ \\
\hline 270 & 3 & $\ldots$ & $15.12 \pm 0.14$ & $13.13 \pm 0.08$ & $10.17 \pm 0.03$ & $8.95 \pm 0.02$ & $7.93 \pm 0.02$ & $6.89 \pm 0.02$ & 0.90 & $\ldots$ \\
\hline 274 & 1 & $16.15 \pm 0.10$ & $15.16 \pm 0.08$ & $14.76 \pm 0.14$ & $13.89 \pm 0.23$ & $12.98 \pm 0.13$ & $\ldots$ & $\ldots$ & 0.68 & $\ldots$ \\
\hline 291 & 3 & $\ldots$ & $16.26 \pm 0.21$ & $13.92 \pm 0.07$ & $9.08 \pm 0.02$ & $7.76 \pm 0.01$ & $6.63 \pm 0.01$ & $5.52 \pm 0.01$ & 1.23 & $\ldots$ \\
\hline 292 & 3 & $\ldots$ & $15.86 \pm 0.17$ & $14.69 \pm 0.11$ & & $12.52 \pm 0.09$ & & & $\ldots$ & $\ldots$ \\
\hline 453 & 1 & $\ldots$ & $15.92 \pm 0.19$ & $13.97 \pm 0.08$ & $11.97 \pm 0.06$ & $10.75 \pm 0.04$ & $9.78 \pm 0.03$ & $8.84 \pm 0.03$ & 0.73 & $\ldots$ \\
\hline 472 & 4 & $\ldots$ & $\ldots$ & $\cdots$ & $12.95 \pm 0.10$ & $11.44 \pm 0.06$ & $10.38 \pm 0.06$ & $9.19 \pm 0.08$ & 1.41 & $\ldots$ \\
\hline 475 & 4 & $\ldots$ & $\ldots$ & $\ldots$ & $13.55 \pm 0.12$ & $12.61 \pm 0.09$ & $11.65 \pm 0.11$ & $10.72 \pm 0.19$ & 0.41 & $\ldots$ \\
\hline 478 & 3 & $\cdots$ & $\cdots$ & $\cdots$ & $11.31 \pm 0.04$ & $9.91 \pm 0.03$ & $8.78 \pm 0.05$ & $7.70 \pm 0.12$ & 1.28 & $\ldots$ \\
\hline 492 & 3 & $\ldots$ & $\ldots$ & $\ldots$ & $12.00 \pm 0.21$ & $11.04 \pm 0.14$ & $\ldots$ & $\ldots$ & 0.90 & $\ldots$ \\
\hline 493 & 3 & $\ldots$ & $\cdots$ & $\cdots$ & $13.40 \pm 0.15$ & $12.24 \pm 0.10$ & $\ldots$ & $\ldots$ & 1.70 & $\ldots$ \\
\hline 559 & 3 & $\cdots$ & $\ldots$ & $14.74 \pm 0.10$ & $13.62 \pm 0.12$ & $12.04 \pm 0.07$ & $10.76 \pm 0.06$ & $10.06 \pm 0.11$ & 1.25 & . \\
\hline 571 & 3 & $\ldots$ & $\ldots$ & $\ldots$ & $11.95 \pm 0.06$ & $10.82 \pm 0.04$ & $9.84 \pm 0.06$ & $9.03 \pm 0.10$ & 0.50 & 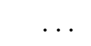 \\
\hline 577 & 3 & $16.31 \pm 0.13$ & $12.87 \pm 0.03$ & $10.98 \pm 0.04$ & $9.16 \pm 0.13$ & $8.17 \pm 0.14$ & $\ldots$ & $\ldots$ & 1.03 & $\ldots$ \\
\hline 581 & 3 & $11.00 \pm 0.02$ & $10.13 \pm 0.02$ & $9.61 \pm 0.02$ & $7.96 \pm 0.04$ & $6.75 \pm 0.03$ & $\ldots$ & $\ldots$ & 1.85 & 0.08 \\
\hline 589 & 3 & $\ldots$ & $\ldots$ & $\ldots$ & $8.08 \pm 0.03$ & $6.79 \pm 0.03$ & $6.03 \pm 0.07$ & $\ldots$ & 1.07 & $\ldots$ \\
\hline
\end{tabular}


Table 8. Photometry of the flat spectrum YSOs in RCW 38.

\begin{tabular}{|c|c|c|c|c|c|c|c|c|c|c|}
\hline $\begin{array}{l}S p . \\
\text { ID }\end{array}$ & $\begin{array}{l}\text { Ep. } \\
\text { ID }\end{array}$ & $\begin{array}{c}\mathrm{J} \\
\text { [mag.] }\end{array}$ & $\begin{array}{c}\mathrm{H} \\
{[\mathrm{mag} .]}\end{array}$ & $\begin{array}{c}\mathrm{K} \\
{[\mathrm{mag} .]}\end{array}$ & $\begin{array}{l}3.6 \mu \mathrm{m} \\
{[\mathrm{mag} .]}\end{array}$ & $\begin{array}{l}4.5 \mu \mathrm{m} \\
\text { [mag.] }\end{array}$ & $\begin{array}{l}5.8 \mu \mathrm{m} \\
{[\mathrm{mag} .]}\end{array}$ & $\begin{array}{l}8.0 \mu \mathrm{m} \\
{[\mathrm{mag} .]}\end{array}$ & $\alpha_{I R A C}$ & $\begin{array}{c}A_{K} \\
{[\mathrm{mag} .]}\end{array}$ \\
\hline 19 & II & & $15.86 \pm 0.13$ & $14.83 \pm 0.12$ & 0.12 & $13.22 \pm 0.12$ & $12.48 \pm 0.13$ & $11.45 \pm 0.16$ & -0.35 & \\
\hline 26 & 4 & $\cdots$ & $15.66 \pm 0.13$ & $14.14 \pm 0.07$ & $\cdots$ & $11.97 \pm 0.07$ & $11.31 \pm 0.08$ & $10.47 \pm 0.13$ & -0.43 & . \\
\hline 42 & II & $\ldots$ & $16.32 \pm 0.18$ & $15.12 \pm 0.14$ & $13.93 \pm 0.14$ & $13.35 \pm 0.13$ & $12.88 \pm 0.16$ & $11.71 \pm 0.19$ & -0.36 & . \\
\hline 52 & 3 & & $15.41 \pm 0.12$ & $14.52 \pm 0.12$ & $13.18 \pm 0.10$ & $12.51 \pm 0.09$ & $11.79 \pm 0.08$ & $10.91 \pm 0.09$ & -0.24 & \\
\hline 64 & II & & $15.64 \pm 0.12$ & $14.44 \pm 0.09$ & $13.58 \pm 0.12$ & $13.03 \pm 0.11$ & $12.19 \pm 0.11$ & $10.80 \pm 0.10$ & 0.37 & \\
\hline 69 & 2 & $16.72 \pm 0.18$ & $15.13 \pm 0.08$ & $14.16 \pm 0.07$ & $12.80 \pm 0.08$ & $12.18 \pm 0.07$ & $11.52 \pm 0.10$ & $10.63 \pm 0.19$ & -0.38 & 0.08 \\
\hline 78 & 2 & $16.37 \pm 0.11$ & $14.86 \pm 0.05$ & $14.17 \pm 0.06$ & $13.73 \pm 0.13$ & $13.16 \pm 0.12$ & $12.68 \pm 0.15$ & $11.36 \pm 0.17$ & -0.19 & . \\
\hline 80 & 4 & & $15.73 \pm 0.14$ & $14.82 \pm 0.13$ & $12.82 \pm 0.08$ & $12.03 \pm 0.07$ & $11.38 \pm 0.07$ & $10.25 \pm 0.07$ & 0.05 & \\
\hline 84 & 2 & $16.85 \pm 0.18$ & $15.97 \pm 0.16$ & $14.81 \pm 0.12$ & $14.24 \pm 0.16$ & $13.92 \pm 0.17$ & $13.00 \pm 0.20$ & $\cdots$ & -0.48 & $\ldots$ \\
\hline 85 & 4 & $13.19 \pm 0.03$ & $11.58 \pm 0.02$ & $10.35 \pm 0.02$ & $8.93 \pm 0.01$ & $8.41 \pm 0.01$ & $7.77 \pm 0.01$ & $6.31 \pm 0.01$ & 0.04 & 0.60 \\
\hline 86 & 4 & $14.91 \pm 0.04$ & $13.13 \pm 0.03$ & $11.78 \pm 0.02$ & $10.11 \pm 0.02$ & $9.38 \pm 0.02$ & $8.59 \pm 0.03$ & $7.22 \pm 0.05$ & 0.34 & 0.76 \\
\hline 92 & 4 & $\ldots$ & $15.40 \pm 0.08$ & $14.77 \pm 0.11$ & $\ldots$ & $13.21 \pm 0.12$ & $\ldots$ & $11.47 \pm 0.38$ & -0.04 & $\ldots$ \\
\hline 95 & 3 & $\cdots$ & $15.92 \pm 0.15$ & $14.81 \pm 0.11$ & $13.08 \pm 0.14$ & $12.44 \pm 0.11$ & $\cdots$ & $\cdots$ & -0.38 & $\ldots$ \\
\hline 97 & 1 & $16.66 \pm 0.16$ & $15.36 \pm 0.07$ & $14.45 \pm 0.09$ & $12.67 \pm 0.08$ & $12.12 \pm 0.08$ & $11.58 \pm 0.27$ & $10.54 \pm 0.47$ & -0.45 & 0.12 \\
\hline 98 & 3 & $16.71 \pm 0.19$ & $14.08 \pm 0.04$ & $12.35 \pm 0.04$ & $10.97 \pm 0.12$ & $10.06 \pm 0.12$ & $\cdots$ & $\cdots$ & 0.68 & \\
\hline 106 & 3 & $\ldots$ & . & $14.28 \pm 0.08$ & $12.46 \pm 0.07$ & $11.68 \pm 0.06$ & $11.02 \pm 0.08$ & $10.20 \pm 0.14$ & -0.28 & \\
\hline 116 & 3 & & $15.70 \pm 0.13$ & $13.20 \pm 0.05$ & $10.62 \pm 0.03$ & $9.54 \pm 0.02$ & $8.76 \pm 0.02$ & $8.08 \pm 0.05$ & 0.03 & \\
\hline 126 & 1 & $13.08 \pm 0.09$ & $11.74 \pm 0.10$ & $10.44 \pm 0.06$ & $8.73 \pm 0.07$ & $7.92 \pm 0.05$ & $\ldots$ & $\ldots$ & 0.34 & $\cdots$ \\
\hline 144 & 3 & & $14.78 \pm 0.08$ & $11.71 \pm 0.05$ & $9.76 \pm 0.16$ & $8.88 \pm 0.11$ & $\ldots$ & $\ldots$ & 0.57 & \\
\hline 153 & 1 & $\cdots$ & $16.16 \pm 0.17$ & $14.54 \pm 0.09$ & $13.28 \pm 0.11$ & $12.74 \pm 0.10$ & $12.21 \pm 0.14$ & $10.80 \pm 0.17$ & -0.04 & $\cdots$ \\
\hline 156 & 3 & $\cdots$ & $15.54 \pm 0.11$ & $13.99 \pm 0.07$ & $12.26 \pm 0.10$ & $11.58 \pm 0.08$ & $11.01 \pm 0.29$ & $\cdots$ & -0.45 & $\ldots$ \\
\hline 164 & 3 & $16.61 \pm 0.14$ & $14.20 \pm 0.04$ & $12.71 \pm 0.05$ & $11.13 \pm 0.05$ & $10.57 \pm 0.04$ & $9.79 \pm 0.12$ & $8.47 \pm 0.17$ & -0.03 & 1.58 \\
\hline 173 & 4 & $\ldots$ & $15.60 \pm 0.12$ & $14.58 \pm 0.09$ & $\ldots$ & $12.53 \pm 0.09$ & $\ldots$ & $10.64 \pm 0.09$ & 0.21 & $\cdots$ \\
\hline 174 & 3 & $\ldots$ & $15.52 \pm 0.11$ & $13.90 \pm 0.06$ & $11.74 \pm 0.07$ & $10.89 \pm 0.05$ & $10.00 \pm 0.15$ & $\ldots$ & 0.47 & \\
\hline 181 & 3 & $\ldots$ & & $13.28 \pm 0.05$ & $11.06 \pm 0.04$ & $10.43 \pm 0.03$ & $9.79 \pm 0.07$ & $8.19 \pm 0.09$ & 0.41 & \\
\hline 182 & 3 & $14.35 \pm 0.04$ & $13.12 \pm 0.03$ & $12.33 \pm 0.04$ & $11.61 \pm 0.07$ & $11.40 \pm 0.07$ & $10.03 \pm 0.20$ & $\cdots$ & 0.06 & 0.41 \\
\hline 183 & 3 & $16.51 \pm 0.13$ & $14.66 \pm 0.07$ & $13.65 \pm 0.07$ & $12.18 \pm 0.07$ & $11.68 \pm 0.06$ & $10.91 \pm 0.17$ & $\ldots$ & -0.60 & 0.57 \\
\hline 187 & 3 & $16.55 \pm 0.17$ & $14.43 \pm 0.05$ & $13.04 \pm 0.04$ & $11.39 \pm 0.05$ & $10.86 \pm 0.04$ & $10.05 \pm 0.09$ & $8.84 \pm 0.14$ & -0.11 & 1.41 \\
\hline 193 & 2 & $16.41 \pm 0.12$ & $14.73 \pm 0.06$ & $13.80 \pm 0.04$ & $13.08 \pm 0.10$ & $12.73 \pm 0.10$ & $12.03 \pm 0.15$ & $10.85 \pm 0.24$ & -0.40 & 0.86 \\
\hline 198 & 3 & $15.60 \pm 0.08$ & $13.63 \pm 0.04$ & $12.42 \pm 0.04$ & $11.15 \pm 0.05$ & $10.57 \pm 0.04$ & $9.89 \pm 0.15$ & $8.56 \pm 0.23$ & -0.07 & 1.19 \\
\hline 201 & 3 & $17.30 \pm 0.24$ & $15.66 \pm 0.11$ & $14.61 \pm 0.09$ & $\cdots$ & $12.91 \pm 0.11$ & $\ldots$ & $10.83 \pm 0.17$ & 0.54 & $\ldots$ \\
\hline 203 & 3 & $15.25 \pm 0.05$ & $13.97 \pm 0.04$ & $13.33 \pm 0.05$ & $12.32 \pm 0.07$ & $11.65 \pm 0.06$ & $10.73 \pm 0.11$ & $9.45 \pm 0.15$ & 0.38 & 0.62 \\
\hline 207 & 1 & $13.36 \pm 0.03$ & $12.21 \pm 0.02$ & $11.01 \pm 0.02$ & $9.34 \pm 0.02$ & $8.67 \pm 0.01$ & $8.03 \pm 0.01$ & $6.47 \pm 0.01$ & 0.40 & $\cdots$ \\
\hline 208 & 3 & $\ldots$ & $14.95 \pm 0.09$ & $13.05 \pm 0.08$ & $11.03 \pm 0.16$ & $10.45 \pm 0.12$ & $\ldots$ & $\ldots$ & -0.61 & $\ldots$ \\
\hline 214 & 4 & $\ldots$ & $15.41 \pm 0.11$ & $14.30 \pm 0.08$ & $13.17 \pm 0.10$ & $12.76 \pm 0.10$ & $12.01 \pm 0.13$ & $10.97 \pm 0.15$ & -0.28 & . \\
\hline 215 & 3 & $16.52 \pm 0.21$ & $14.70 \pm 0.09$ & $13.65 \pm 0.09$ & $12.08 \pm 0.09$ & $11.79 \pm 0.11$ & $9.88 \pm 0.15$ & $\ldots$ & 1.39 & . \\
\hline 220 & 3 & . & $14.74 \pm 0.14$ & $13.32 \pm 0.06$ & $\cdots$ & $10.75 \pm 0.04$ & $\cdots$ & $\ldots$ & $\cdots$ & . \\
\hline 227 & 3 & .. & $15.80 \pm 0.13$ & $13.81 \pm 0.06$ & $11.02 \pm 0.04$ & $10.32 \pm 0.03$ & $9.64 \pm 0.05$ & $8.58 \pm 0.11$ & -0.07 & 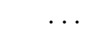 \\
\hline 230 & 4 & $\cdots$ & .. & $13.71 \pm 0.08$ & $11.09 \pm 0.15$ & & $\ldots$ & $\ldots$ & $\ldots$ & . \\
\hline 240 & 4 & & $15.57 \pm 0.11$ & $14.40 \pm 0.08$ & $\cdots$ & $12.16 \pm 0.07$ & $\cdots$ & $10.12 \pm 0.15$ & 0.48 & $\cdots$ \\
\hline 260 & 3 & $16.08 \pm 0.09$ & $15.15 \pm 0.08$ & $14.64 \pm 0.11$ & $13.91 \pm 0.15$ & $13.37 \pm 0.13$ & $12.91 \pm 0.36$ & $11.66 \pm 0.68$ & -0.33 & \\
\hline 266 & 4 & $\cdots$ & $15.66 \pm 0.11$ & $14.00 \pm 0.07$ & $11.10 \pm 0.04$ & $10.12 \pm 0.03$ & $9.31 \pm 0.03$ & $8.40 \pm 0.02$ & 0.22 & \\
\hline 271 & 3 & $\ldots$ & $15.48 \pm 0.13$ & $13.78 \pm 0.07$ & $11.75 \pm 0.06$ & $11.03 \pm 0.05$ & $10.20 \pm 0.15$ & $\ldots$ & 0.10 & $\ldots$ \\
\hline 306 & 3 & . & $15.98 \pm 0.16$ & $14.77 \pm 0.11$ & $13.15 \pm 0.10$ & $12.65 \pm 0.09$ & $11.99 \pm 0.19$ & $10.70 \pm 0.15$ & -0.04 & 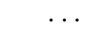 \\
\hline 316 & 1 & $\ldots$ & $\ldots$ & $14.90 \pm 0.13$ & $12.60 \pm 0.08$ & $11.83 \pm 0.06$ & $11.10 \pm 0.06$ & $10.10 \pm 0.07$ & 0.01 & \\
\hline
\end{tabular}


Table 8-Continued

\begin{tabular}{|c|c|c|c|c|c|c|c|c|c|c|}
\hline $\begin{array}{l}S p . \\
\text { ID }\end{array}$ & $\begin{array}{l}\text { Ep. } \\
\text { ID }\end{array}$ & $\begin{array}{c}\mathrm{J} \\
{[\mathrm{mag} .]}\end{array}$ & $\begin{array}{c}\mathrm{H} \\
{[\mathrm{mag} .]}\end{array}$ & $\begin{array}{c}\mathrm{K} \\
{[\mathrm{mag} .]}\end{array}$ & $\begin{array}{l}3.6 \mu \mathrm{m} \\
\text { [mag.] }\end{array}$ & $\begin{array}{l}4.5 \mu \mathrm{m} \\
{[\mathrm{mag} .]}\end{array}$ & $\begin{array}{l}5.8 \mu \mathrm{m} \\
\text { [mag.] }\end{array}$ & $\begin{array}{l}8.0 \mu \mathrm{m} \\
\text { [mag.] }\end{array}$ & $\alpha_{I R A C}$ & $\begin{array}{c}A_{K} \\
{[\mathrm{mag} .]}\end{array}$ \\
\hline 324 & 3 & $\ldots$ & $14.07 \pm 0.07$ & $12.52 \pm 0.06$ & $10.88 \pm 0.04$ & $10.03 \pm 0.03$ & $9.28 \pm 0.05$ & $8.18 \pm 0.09$ & 0.10 & 0.74 \\
\hline 325 & 3 & $\ldots$ & & $14.09 \pm 0.13$ & $12.27 \pm 0.22$ & $11.55 \pm 0.19$ & $\ldots$ & $\cdots$ & -0.03 & $\ldots$ \\
\hline 344 & 1 & $16.81 \pm 0.18$ & $14.91 \pm 0.07$ & $13.65 \pm 0.05$ & $12.08 \pm 0.07$ & $11.38 \pm 0.05$ & $10.37 \pm 0.07$ & $9.18 \pm 0.08$ & 0.46 & 0.49 \\
\hline 346 & 4 & $\ldots$ & $16.04 \pm 0.17$ & $14.57 \pm 0.11$ & $12.76 \pm 0.11$ & $12.21 \pm 0.09$ & $11.37 \pm 0.43$ & $\ldots$ & -0.20 & $\ldots$ \\
\hline 351 & 3 & $\ldots$ & $16.05 \pm 0.17$ & $13.96 \pm 0.07$ & $11.92 \pm 0.25$ & $11.13 \pm 0.22$ & $\ldots$ & $\ldots$ & 0.22 & $\ldots$ \\
\hline 352 & 3 & $16.11 \pm 0.11$ & $\ldots$ & - & $11.04 \pm 0.04$ & $10.51 \pm 0.04$ & $9.74 \pm 0.13$ & $8.59 \pm 0.20$ & -0.02 & $\ldots$ \\
\hline 353 & 3 & ... & $15.33 \pm 0.09$ & $13.90 \pm 0.06$ & $12.09 \pm 0.07$ & $11.42 \pm 0.06$ & $10.82 \pm 0.21$ & $9.78 \pm 0.55$ & -0.40 & 1.06 \\
\hline 356 & 2 & $16.06 \pm 0.10$ & $15.14 \pm 0.08$ & $14.98 \pm 0.16$ & $14.62 \pm 0.24$ & $13.80 \pm 0.23$ & $\ldots$ & $\ldots$ & 0.36 & $\ldots$ \\
\hline 362 & 3 & $\ldots$ & $16.06 \pm 0.17$ & $14.83 \pm 0.12$ & $13.07 \pm 0.12$ & $12.40 \pm 0.09$ & $11.23 \pm 0.26$ & .. & 0.67 & $\ldots$ \\
\hline 381 & 3 & $\ldots$ & $15.71 \pm 0.13$ & $14.23 \pm 0.07$ & $12.36 \pm 0.08$ & $11.81 \pm 0.07$ & $10.33 \pm 0.10$ & $\ldots$ & 1.04 & $\ldots$ \\
\hline 382 & 3 & $14.06 \pm 0.03$ & $12.90 \pm 0.03$ & $12.43 \pm 0.02$ & $11.98 \pm 0.06$ & $11.71 \pm 0.06$ & $11.10 \pm 0.08$ & $9.77 \pm 0.09$ & -0.38 & 0.59 \\
\hline 388 & 3 & $16.51 \pm 0.14$ & $14.75 \pm 0.06$ & $13.69 \pm 0.06$ & $11.86 \pm 0.28$ & $11.15 \pm 0.14$ & $\ldots$ & $\ldots$ & -0.08 & $\ldots$ \\
\hline 396 & 3 & $16.73 \pm 0.19$ & $15.09 \pm 0.08$ & $13.91 \pm 0.07$ & $12.99 \pm 0.14$ & $12.58 \pm 0.11$ & $11.33 \pm 0.28$ & $\ldots$ & 0.34 & $\ldots$ \\
\hline 403 & II & $\ldots$ & $15.00 \pm 0.08$ & $13.60 \pm 0.08$ & $11.59 \pm 0.36$ & $10.73 \pm 0.25$ & $\ldots$ & $\ldots$ & $\ldots$ & $\ldots$ \\
\hline 404 & 3 & $\ldots$ & $\ldots$ & $14.70 \pm 0.11$ & $12.03 \pm 0.06$ & $11.20 \pm 0.05$ & $10.46 \pm 0.08$ & $9.52 \pm 0.16$ & 0.01 & $\ldots$ \\
\hline 414 & 3 & $\ldots$ & $15.54 \pm 0.17$ & $12.94 \pm 0.06$ & $9.85 \pm 0.17$ & $9.25 \pm 0.21$ & $\ldots$ & $\ldots$ & -0.50 & $\ldots$ \\
\hline 434 & 1 & $15.34 \pm 0.06$ & $13.83 \pm 0.04$ & $12.88 \pm 0.03$ & $11.83 \pm 0.05$ & $11.22 \pm 0.05$ & $10.69 \pm 0.06$ & $9.56 \pm 0.07$ & -0.40 & 0.70 \\
\hline 438 & 1 & $16.81 \pm 0.19$ & $15.61 \pm 0.12$ & $14.31 \pm 0.09$ & $\ldots$ & $12.75 \pm 0.10$ & $12.30 \pm 0.12$ & $11.31 \pm 0.12$ & -0.49 & $\ldots$ \\
\hline 443 & 1 & $16.47 \pm 0.13$ & $14.94 \pm 0.07$ & $14.04 \pm 0.06$ & $\ldots$ & $12.65 \pm 0.09$ & $\ldots$ & $11.13 \pm 0.10$ & -0.43 & 0.63 \\
\hline 459 & 3 & $15.46 \pm 0.07$ & $14.24 \pm 0.05$ & $13.56 \pm 0.05$ & $12.82 \pm 0.09$ & $12.39 \pm 0.08$ & $11.60 \pm 0.18$ & $10.10 \pm 0.22$ & 0.22 & 0.49 \\
\hline 460 & 3 & $16.68 \pm 0.16$ & $15.52 \pm 0.13$ & $14.45 \pm 0.09$ & $\ldots$ & $12.92 \pm 0.11$ & $\ldots$ & $11.12 \pm 0.33$ & 0.07 & $\ldots$ \\
\hline 466 & 1 & $\ldots$ & $\ldots$ & $\ldots$ & $13.45 \pm 0.11$ & $12.63 \pm 0.09$ & $11.83 \pm 0.09$ & $10.78 \pm 0.09$ & 0.20 & $\ldots$ \\
\hline 467 & 1 & $\ldots$ & $\ldots$ & $\ldots$ & $11.72 \pm 0.05$ & $11.16 \pm 0.05$ & $10.52 \pm 0.05$ & $9.62 \pm 0.06$ & -0.45 & $\ldots$ \\
\hline 471 & II & $\ldots$ & $\ldots$ & $\ldots$ & $12.74 \pm 0.08$ & $11.79 \pm 0.06$ & $11.07 \pm 0.08$ & $10.44 \pm 0.16$ & -0.24 & $\ldots$ \\
\hline 477 & 3 & $\ldots$ & $\ldots$ & $\ldots$ & $14.29 \pm 0.18$ & $13.30 \pm 0.14$ & $13.03 \pm 0.36$ & & -0.46 & $\ldots$ \\
\hline 481 & 1 & $\ldots$ & $\ldots$ & $\ldots$ & $11.68 \pm 0.05$ & $10.75 \pm 0.04$ & $10.02 \pm 0.04$ & $9.21 \pm 0.04$ & -0.04 & $\ldots$ \\
\hline 490 & 3 & $\ldots$ & $\ldots$ & $\cdots$ & $12.66 \pm 0.09$ & $11.50 \pm 0.06$ & $11.23 \pm 0.29$ & $\ldots$ & -0.12 & $\ldots$ \\
\hline 502 & 2 & $16.45 \pm 0.11$ & $15.38 \pm 0.09$ & $14.70 \pm 0.09$ & $\ldots$ & $13.26 \pm 0.12$ & $\ldots$ & $11.56 \pm 0.22$ & -0.08 & $\ldots$ \\
\hline 505 & 3 & $\ldots$ & $15.95 \pm 0.14$ & $15.36 \pm 0.20$ & $14.37 \pm 0.17$ & $13.75 \pm 0.16$ & $\ldots$ & $\ldots$ & -0.45 & $\ldots$ \\
\hline 516 & 3 & $16.14 \pm 0.12$ & $14.31 \pm 0.05$ & $13.17 \pm 0.03$ & $11.38 \pm 0.04$ & $10.60 \pm 0.04$ & $10.00 \pm 0.07$ & $9.15 \pm 0.14$ & -0.34 & $\ldots$ \\
\hline 524 & 2 & $16.39 \pm 0.13$ & $15.25 \pm 0.09$ & $14.34 \pm 0.09$ & $12.41 \pm 0.07$ & $11.65 \pm 0.06$ & $10.88 \pm 0.05$ & $10.10 \pm 0.05$ & -0.19 & $\ldots$ \\
\hline 531 & 2 & $14.34 \pm 0.05$ & $13.20 \pm 0.05$ & $12.70 \pm 0.04$ & $\cdots$ & $10.55 \pm 0.04$ & $\ldots$ & $8.53 \pm 0.04$ & 0.39 & 0.52 \\
\hline 536 & 2 & $16.49 \pm 0.13$ & $15.23 \pm 0.10$ & $14.73 \pm 0.13$ & $13.72 \pm 0.13$ & $13.18 \pm 0.12$ & $12.49 \pm 0.12$ & $11.44 \pm 0.13$ & -0.22 & $\ldots$ \\
\hline 543 & 3 & $15.51 \pm 0.09$ & $13.65 \pm 0.06$ & $12.68 \pm 0.06$ & $11.47 \pm 0.05$ & $11.18 \pm 0.05$ & $\ldots$ & $\ldots$ & -2.22 & 1.23 \\
\hline 561 & 3 & $\ldots$ & $16.13 \pm 0.21$ & $14.51 \pm 0.12$ & 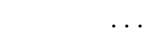 & $11.91 \pm 0.13$ & $\ldots$ & $\ldots$ & $\cdots$ & $\ldots$ \\
\hline 572 & 4 & $\ldots$ & $\cdots$ & $\cdots$ & $11.87 \pm 0.05$ & $11.14 \pm 0.05$ & $10.54 \pm 0.05$ & $9.58 \pm 0.06$ & -0.26 & $\cdots$ \\
\hline 573 & 3 & $13.63 \pm 0.02$ & $11.22 \pm 0.02$ & $9.31 \pm 0.02$ & $7.04 \pm 0.01$ & $6.29 \pm 0.01$ & $5.53 \pm 0.01$ & $4.56 \pm 0.02$ & -0.20 & 1.24 \\
\hline 585 & 3 & $\ldots$ & $14.94 \pm 0.06$ & $11.51 \pm 0.02$ & $7.50 \pm 0.01$ & $6.38 \pm 0.01$ & $5.42 \pm 0.01$ & $4.65 \pm 0.01$ & -0.32 & 4.64 \\
\hline 590 & 3 & $\ldots$ & $\ldots$ & $\ldots$ & $\ldots$ & $11.18 \pm 0.05$ & $10.31 \pm 0.11$ & $\ldots$ & 0.43 & $\ldots$ \\
\hline 598 & 4 & $\ldots$ & $\ldots$ & $\ldots$ & \pm 0.17 & $12.30 \pm 0.08$ & $11.33 \pm 0.11$ & \pm 0.38 & $\ldots$ & $\ldots$ \\
\hline 756 & 1 & $\cdots$ & $\ldots$ & $\cdots$ & $14.59 \pm 0.20$ & $13.46 \pm 0.13$ & $12.62 \pm 0.15$ & $11.63 \pm 0.25$ & $\ldots$ & $\ldots$ \\
\hline 762 & 1 & $\ldots$ & $\ldots$ & $\ldots$ & $14.47 \pm 0.18$ & $13.26 \pm 0.12$ & $12.37 \pm 0.12$ & $11.35 \pm 0.15$ & $\ldots$ & $\ldots$ \\
\hline 774 & 4 & $\cdots$ & $\ldots$ & $\ldots$ & $13.53 \pm 0.12$ & $12.65 \pm 0.09$ & $11.91 \pm 0.13$ & $\ldots$ & $\ldots$ & $\ldots$ \\
\hline 813 & 4 & $\ldots$ & $\ldots$ & $9.57 \pm 0.07$ & $8.42 \pm 0.05$ & $7.68 \pm 0.09$ & $6.87 \pm 0.12$ & . & $\ldots$ & $\cdots$ \\
\hline 814 & 4 & $\ldots$ & $\ldots$ & $\ldots$ & $8.94 \pm 0.05$ & $7.88 \pm 0.06$ & $7.10 \pm 0.14$ & .. & $\ldots$ & $\ldots$ \\
\hline
\end{tabular}


Table 8-Continued

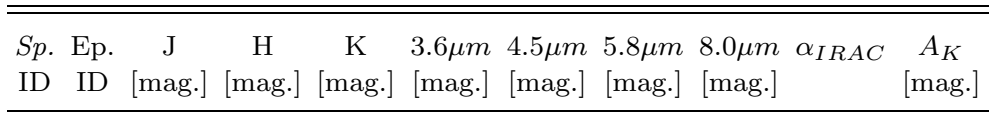

Table 9. Photometry of the Class II YSOs in RCW 38.

\begin{tabular}{|c|c|c|c|c|c|c|c|c|c|c|}
\hline $\begin{array}{l}S p . \\
\text { ID }\end{array}$ & $\begin{array}{l}\text { Ep. } \\
\text { ID }\end{array}$ & $\begin{array}{c}\mathrm{J} \\
{[\mathrm{mag} .]}\end{array}$ & $\begin{array}{c}\mathrm{H} \\
{[\mathrm{mag} .]}\end{array}$ & $\begin{array}{c}\mathrm{K} \\
{[\mathrm{mag} .]}\end{array}$ & $\begin{array}{l}3.6 \mu \mathrm{m} \\
\text { [mag.] }\end{array}$ & $\begin{array}{l}4.5 \mu \mathrm{m} \\
{[\mathrm{mag} .]}\end{array}$ & $\begin{array}{l}5.8 \mu \mathrm{m} \\
\text { [mag.] }\end{array}$ & $\begin{array}{l}8.0 \mu \mathrm{m} \\
{[\mathrm{mag} .]}\end{array}$ & $C$ & $\begin{array}{c}A_{K} \\
{[\mathrm{mag} .]}\end{array}$ \\
\hline 1 & 4 & & .11 & 09 & .11 & .11 & $=0.13$ & $11.68 \pm 0.16$ & 4 & \\
\hline 2 & 3 & $.59 \pm 0.07$ & $14.10 \pm$ & & & 12.9 & & & & 0.9 \\
\hline 3 & 3 & $.55 \pm 0.07$ & \pm 0.05 & $13.01 \pm$ & $11.89 \pm 0.06$ & 11.49 & $28 \pm 0.07$ & 12 & & 1.01 \\
\hline 4 & 4 & & $15.46 \pm 0.14$ & $14.39 \pm 0.08$ & & 12.31 & & 0.24 & & \\
\hline 5 & 2 & \pm 0.22 & $15.73 \pm 0.14$ & $14.97 \pm 0.14$ & $13.51 \pm 0.12$ & $13.00 \pm 0.11$ & 50.14 & $12.45 \pm 0.36$ & & \\
\hline 6 & 4 & $98 \pm 0.20$ & $.13 \pm 0.08$ & $14.28 \pm 0.08$ & & 12.28 & & 10.9 & & \\
\hline 7 & 3 & & $14.26 \pm$ & $12.71 \pm$ & $10.93=$ & 10.25 & & & & 1.51 \\
\hline 8 & 2 & $86 \pm 0.09$ & $.51 \pm$ & $13.82=$ & $12.89 \pm 0.09$ & 12.49 & $12.14 \pm 0.13$ & 0.19 & & 0.67 \\
\hline 9 & 4 & $.05 \pm 0.10$ & 06 & $13.47 \pm$ & & $11.6^{7}$ & & & & 0.2 \\
\hline 10 & 2 & & $15.55 \pm 0.13$ & $14.25 \pm$ & \pm 0.08 & 12.1 & $=0.08$ & & & \\
\hline 11 & 4 & $=0.11$ & 13.31 & $12.53 \pm$ & & & & & & \\
\hline 12 & 3 & & $15.68 \pm$ & $14.45 \pm 0.08$ & 0.09 & 12.3 & 0.11 & & & 1.20 \\
\hline 13 & 2 & $5.04 \pm 0.05$ & $13.84\rfloor$ & $13.20 \pm 0.04$ & $\ldots$ & 12.0 & \pm 0.09 & & & 0.49 \\
\hline 14 & 4 & $\ldots$ & $15.66 \pm 0.10$ & 15.01 & ... & 13.2 & & .21 & & \\
\hline 15 & 2 & & 15.3 & 14.8 & 0.13 & & 0.16 & & & \\
\hline 16 & 4 & \pm 0.12 & 14.7 & 13.7 & 12.4 & 11. & & & & 0.34 \\
\hline 18 & 4 & \pm 0.04 & & & $\ldots$ & & & & & 0.75 \\
\hline 20 & 2 & $.75 \pm 0.19$ & 11 & 14.5 & 0.10 & & 0.10 & .12 & & \\
\hline 21 & 2 & & 14.7 & 12.7 & 03 & & .03 & 0.06 & & 2.60 \\
\hline 22 & 3 & \pm 0.08 & 14.87 & 14.25 & 0.12 & 12. & 0.25 & & & 0.40 \\
\hline 23 & 2 & $\ldots$ & & & 09 & & .11 & .15 & & 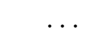 \\
\hline 24 & 3 & $\ldots$ & 15. & 5 & 09 & & .09 & & & \\
\hline 25 & 2 & $.35 \pm 0.10$ & 140 & 14.6 & 0.13 & & 0.15 & 16 & & \\
\hline 27 & 4 & 0.15 & & & & & & & & \\
\hline 28 & 3 & $3 \pm 0.14$ & & & & & 0.07 & $=0.11$ & & 0.58 \\
\hline 29 & 3 & 0.13 & & & 12 . & & 0.13 & $\ldots$ & & 1.38 \\
\hline 30 & 3 & 0.21 & 0 & & 12. & 11. & $\ldots$ & $\ldots$ & & 1.32 \\
\hline 31 & 3 & \pm 0.02 & 03 & 2 & 0.01 & & $=0.01$ & 0.01 & & $0.6^{\prime}$ \\
\hline 32 & 3 & $.52 \pm 0.03$ & & & $11.23 \pm 0.04$ & & $=0.04$ & $=0.06$ & -1 & 0.87 \\
\hline 33 & 1 & 20 & & & $\ldots$ & & $\ldots$ & $\ldots$ & & .. \\
\hline 34 & 2 & 04 & 55 & 3 & . & & $\ldots$ & 0.06 & & 0.45 \\
\hline 35 & 2 & $.96 \pm 0.04$ & 0.05 & 11.22 & $=0.03$ & & 0.03 & & & 0.29 \\
\hline 37 & 3 & $46 \pm 0.14$ & 0.04 & 12. & 10. & 10. & .03 & 0.06 & & 1.8 \\
\hline 38 & 2 & & & & & & & $=0.05$ & & 1.40 \\
\hline 39 & 3 & .19 & .13 & 0.12 & \pm 0.16 & 13.7 & 0.28 & $\ldots$ & & .. \\
\hline 40 & 2 & $2.01 \pm 0.02$ & 0.02 & & $8.91 \pm 0.01$ & & \pm 0.01 & $=0.01$ & & 0.04 \\
\hline 41 & 3 & \pm 0.05 & .02 & 12. & $12.18 \pm 0.06$ & 11. & $11.55 \pm 0.09$ & 0.12 & & 1.1 \\
\hline 43 & 4 & & & & & & .. & $=0.15$ & & 0.0 \\
\hline 44 & 3 & .21 & & & 0.10 & & $\ldots$ & 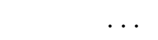 & & 2.1 \\
\hline 45 & 2 & $.50 \pm 0.15$ & 0.08 & $14.69 \pm 0.12$ & $=0.12$ & 13.3 & 0.16 & $\ldots$ & & . \\
\hline 46 & 3 & $.71 \pm 0.04$ & $.89 \pm 0.03$ & $13.73 \pm 0.05$ & $13.37 \pm 0.11$ & 0.13 & $13.33 \pm 0.21$ & $\ldots$ & 37 & 0.23 \\
\hline 47 & 2 & $4.70 \pm 0.03$ & $13.56 \pm 0.03$ & $13.06 \pm 0.03$ & $12.61 \pm 0.08$ & 12.3 & $11.99 \pm 0.10$ & & & 0.5 \\
\hline 48 & 3 & 0.12 & & & 12.9 & 12.5 & 11.9 & 10. & & 0.82 \\
\hline 49 & 3 & $1.10 \pm 0.02$ & $10.44 \pm 0.03$ & $10.02 \pm 0.02$ & $9.80 \pm 0.02$ & $9.58 \pm 0.03$ & $9.12 \pm 0.05$ & $7.75 \pm 0.11$ & -0.52 & 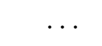 \\
\hline 50 & 4 & $16.10 \pm 0.09$ & $14.43 \pm 0.04$ & $13.49 \pm 0.04$ & . & $12.18 \pm 0.07$ & 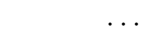 & $11.04 \pm 0.20$ & -1.08 & 0.9 \\
\hline
\end{tabular}


Table 9 - Continued

\begin{tabular}{|c|c|c|c|c|c|c|c|c|c|c|}
\hline $\begin{array}{l}S p . \\
\text { ID }\end{array}$ & $\begin{array}{l}\text { Ep. } \\
\text { ID }\end{array}$ & $\begin{array}{c}\mathrm{J} \\
{[\mathrm{mag} .]}\end{array}$ & $\begin{array}{c}\mathrm{H} \\
{[\mathrm{mag} .]}\end{array}$ & $\begin{array}{c}\mathrm{K} \\
{[\mathrm{mag} .]}\end{array}$ & $\begin{array}{l}3.6 \mu \mathrm{m} \\
\text { [mag.] }\end{array}$ & $\begin{array}{l}4.5 \mu \mathrm{m} \\
{[\mathrm{mag} .]}\end{array}$ & $\begin{array}{l}5.8 \mu \mathrm{m} \\
\text { [mag.] }\end{array}$ & $\begin{array}{l}8.0 \mu \mathrm{m} \\
{[\mathrm{mag} .]}\end{array}$ & $\alpha_{I R A C}$ & $\begin{array}{c}A_{K} \\
{[\mathrm{mag} .]}\end{array}$ \\
\hline 51 & 4 & $16.63 \pm 0.17$ & $14.65 \pm 0.08$ & $13.37 \pm 0.06$ & $11.91 \pm 0.14$ & $11.51 \pm 0.11$ & & . & -1.29 & \\
\hline 53 & 2 & $15.00 \pm 0.05$ & $14.29 \pm 0.05$ & $13.87 \pm 0.05$ & $13.22 \pm 0.10$ & $12.93 \pm 0.11$ & $12.63 \pm 0.16$ & $\ldots$ & -1.75 & $\cdots$ \\
\hline 54 & 3 & $16.77 \pm 0.18$ & $15.25 \pm 0.08$ & $14.47 \pm 0.09$ & $13.36 \pm 0.11$ & $12.98 \pm 0.11$ & $12.74 \pm 0.14$ & $11.84 \pm 0.16$ & -1.18 & $\ldots$ \\
\hline 55 & 3 & $14.93 \pm 0.05$ & $12.87 \pm 0.04$ & $11.58 \pm 0.02$ & $10.25 \pm 0.03$ & $9.75 \pm 0.02$ & $9.30 \pm 0.03$ & $8.42 \pm 0.03$ & -0.98 & 1.24 \\
\hline 56 & 2 & $14.52 \pm 0.04$ & $13.32 \pm 0.03$ & $12.72 \pm 0.03$ & $12.09 \pm 0.06$ & $11.77 \pm 0.06$ & $11.47 \pm 0.07$ & $10.85 \pm 0.10$ & -1.56 & 0.54 \\
\hline 57 & 2 & $16.52 \pm 0.13$ & $14.87 \pm 0.07$ & $13.82 \pm 0.07$ & $12.54 \pm 0.07$ & $11.87 \pm 0.06$ & $11.58 \pm 0.09$ & $10.70 \pm 0.13$ & -0.84 & 0.04 \\
\hline 58 & 4 & & $15.06 \pm 0.06$ & $13.88 \pm 0.06$ & $12.92 \pm 0.09$ & $12.58 \pm 0.09$ & $12.40 \pm 0.13$ & $11.73 \pm 0.15$ & -1.80 & 1.53 \\
\hline 59 & 4 & $14.77 \pm 0.02$ & $12.99 \pm 0.03$ & $11.81 \pm 0.02$ & & $9.76 \pm 0.02$ & $9.35 \pm 0.03$ & $8.56 \pm 0.03$ & -0.96 & 0.91 \\
\hline 60 & 4 & $16.84 \pm 0.21$ & $15.55 \pm 0.13$ & $14.57 \pm 0.11$ & $13.38 \pm 0.11$ & $12.90 \pm 0.11$ & $12.95 \pm 0.23$ & 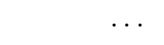 & -2.06 & $\ldots$ \\
\hline 61 & 2 & $17.17 \pm 0.22$ & $15.79 \pm 0.12$ & $14.86 \pm 0.12$ & $13.71 \pm 0.13$ & $13.22 \pm 0.12$ & $12.72 \pm 0.13$ & $11.82 \pm 0.13$ & -0.70 & $\ldots$ \\
\hline 62 & 2 & $14.47 \pm 0.03$ & $12.47 \pm 0.03$ & $10.94 \pm 0.02$ & $9.28 \pm 0.02$ & $8.72 \pm 0.02$ & $8.25 \pm 0.02$ & $7.75 \pm 0.02$ & -1.27 & 0.95 \\
\hline 63 & 3 & $15.46 \pm 0.07$ & $13.75 \pm 0.04$ & $12.92 \pm 0.04$ & $12.22 \pm 0.06$ & $12.09 \pm 0.07$ & $11.44 \pm 0.19$ & & -1.67 & 1.12 \\
\hline 66 & 4 & $16.58 \pm 0.14$ & $14.83 \pm 0.12$ & $14.18 \pm 0.09$ & & $12.18 \pm 0.07$ & & $11.29 \pm 0.16$ & -1.42 & $\ldots$ \\
\hline 67 & 3 & $\ldots$ & $15.36 \pm 0.09$ & $14.05 \pm 0.07$ & $12.83 \pm 0.08$ & $12.34 \pm 0.08$ & $11.87 \pm 0.12$ & $11.03 \pm 0.22$ & -1.03 & 1.36 \\
\hline 68 & 4 & $16.72 \pm 0.16$ & $15.15 \pm 0.08$ & $14.58 \pm 0.09$ & & $13.14 \pm 0.12$ & & $12.07 \pm 0.15$ & & $\ldots$ \\
\hline 70 & 2 & $15.91 \pm 0.08$ & $14.49 \pm 0.07$ & $13.68 \pm 0.06$ & $12.39 \pm 0.07$ & $12.02 \pm$ & $11.73 \pm 0.08$ & $11.37 \pm 0.13$ & -1 & 0.69 \\
\hline 71 & 2 & $15.23 \pm 0.06$ & $13.70 \pm 0.04$ & $12.80 \pm 0.04$ & $11.81 \pm 0.05$ & $11.47 \pm 0.05$ & $11.31 \pm 0.11$ & $10.98 \pm 0.32$ & -2.08 & 0.76 \\
\hline 73 & 2 & $\cdots$ & $15.10 \pm 0.08$ & $14.28 \pm 0.10$ & & $12.55 \pm 0.09$ & $12.07 \pm 0.10$ & $11.23 \pm 0.12$ & -0.71 & $\ldots$ \\
\hline 74 & 3 & $\ldots$ & $15.64 \pm 0.12$ & $14.50 \pm 0.10$ & $12.87 \pm 0.09$ & 12.26 & $11.83 \pm 0.09$ & $11.12 \pm 0.12$ & & $\ldots$ \\
\hline 75 & 4 & $16.24 \pm 0.09$ & $14.72 \pm 0.04$ & $13.84 \pm 0.04$ & $12.81 \pm 0.09$ & $12.24 \pm 0.08$ & $11.84 \pm 0.09$ & $10.95 \pm 0.09$ & -0.90 & 0.78 \\
\hline 76 & 3 & & $15.52 \pm 0.12$ & $14.20 \pm 0.07$ & $12.40 \pm 0.07$ & $11.79 \pm 0.06$ & $11.33 \pm 0.07$ & $10.67 \pm 0.08$ & -0.91 & $\ldots$ \\
\hline 79 & 1 & $12.85 \pm 0.02$ & $12.30 \pm 0.03$ & 12.14 & $12.04 \pm 0.06$ & 12.0 & $11.89 \pm 0.09$ & 0.15 & -2.44 & $\cdots$ \\
\hline 81 & 4 & $16.36 \pm 0.11$ & $15.28 \pm 0.07$ & $14.47 \pm 0.08$ & $\ldots$ & $13.08 \pm 0.11$ & $\ldots$ & $11.88 \pm 0.16$ & -0.92 & $\ldots$ \\
\hline 83 & 3 & $16.31 \pm 0.13$ & $14.85 \pm 0.07$ & $13.84 \pm 0.05$ & $12.98 \pm 0.09$ & $12.56 \pm 0.09$ & $12.04 \pm 0.11$ & $10.84 \pm 0.12$ & -0.55 & 0.86 \\
\hline 87 & 2 & $13.78 \pm 0.03$ & $12.68 \pm 0.03$ & $11.79 \pm 0.03$ & $10.44 \pm 0.03$ & $10.06 \pm 0.03$ & $9.70 \pm 0.03$ & $9.00 \pm 0.03$ & -1.26 & 0.10 \\
\hline 88 & 4 & $15.16 \pm 0.05$ & $13.76 \pm 0.04$ & $12.74 \pm 0.04$ & & $11.23 \pm 0.05$ & $\cdots$ & $\cdots$ & & 0.47 \\
\hline 89 & 3 & $\ldots$ & $14.77 \pm 0.07$ & $13.59 \pm 0.06$ & $12.87 \pm 0.09$ & $12.41 \pm 0.09$ & & & -1.47 & 1.10 \\
\hline 90 & 2 & $\cdots$ & $15.56 \pm 0.12$ & $15.09 \pm 0.14$ & $13.93 \pm 0.14$ & $13.56 \pm 0.14$ & $13.26 \pm 0.17$ & $12.66 \pm 0.22$ & -1.45 & $\ldots$ \\
\hline 91 & 1 & $17.13 \pm 0.26$ & $15.50 \pm 0.11$ & $14.82 \pm 0.14$ & $13.55 \pm 0.13$ & $13.02 \pm 0.12$ & $13.28 \pm 0.63$ & $\ldots$ & -2.37 & $\ldots$ \\
\hline 94 & 4 & & $15.03 \pm 0.09$ & $13.84 \pm 0.07$ & $12.90 \pm 0.10$ & $12.59 \pm 0.10$ & $\ldots$ & $\ldots$ & -2.29 & 1.65 \\
\hline 96 & 2 & $15.71 \pm 0.08$ & $13.91 \pm 0.05$ & $12.70 \pm 0.04$ & $11.46 \pm 0.05$ & $10.87 \pm 0.04$ & $10.51 \pm 0.05$ & $9.57 \pm 0.06$ & -0.90 & 0.92 \\
\hline 101 & 1 & $16.68 \pm 0.17$ & $15.20 \pm 0.09$ & $14.57 \pm 0.11$ & $13.20 \pm 0.10$ & $12.79 \pm 0.10$ & $12.39 \pm 0.15$ & $\ldots$ & -1.33 & $\ldots$ \\
\hline 102 & 3 & $\cdots$ & $16.19 \pm 0.20$ & $14.92 \pm 0.13$ & $13.71 \pm 0.29$ & $13.37 \pm 0.26$ & $\cdots$ & $\cdots$ & -1.53 & $\cdots$ \\
\hline 103 & 3 & 12 . & $11.53 \pm 0.03$ & $10.76 \pm 0.02$ & $9.77 \pm 0.02$ & $9.33 \pm 0.02$ & $=0.03$ & 0.06 & -1.02 & 0.12 \\
\hline 104 & 3 & $16.17 \pm 0.12$ & $14.05 \pm 0.07$ & $12.77 \pm 0.05$ & $11.49 \pm 0.05$ & $10.92 \pm 0.04$ & $10.33 \pm 0.04$ & $9.36 \pm 0.05$ & -0.59 & 1.01 \\
\hline 105 & 3 & $15.80 \pm 0.08$ & $14.32 \pm 0.04$ & $13.12 \pm 0.03$ & $12.39 \pm 0.08$ & $11.88 \pm 0.07$ & $\ldots$ & & -1.04 & 0.41 \\
\hline 107 & 1 & $17.10 \pm 0.21$ & $15.88 \pm 0.16$ & $15.03 \pm 0.15$ & $13.61 \pm 0.12$ & $12.86 \pm 0.10$ & $12.73 \pm 0.15$ & $11.80 \pm 0.18$ & -0.93 & $\ldots$ \\
\hline 108 & 1 & & $15.37 \pm 0.16$ & $15.12 \pm 0.15$ & $14.01 \pm 0.15$ & $13.69 \pm 0.16$ & $\ldots$ & $\ldots$ & -1.62 & $\ldots$ \\
\hline 110 & 4 & $16.40 \pm 0.12$ & $14.83 \pm 0.04$ & $14.33 \pm 0.08$ & $\ldots$ & $12.99 \pm 0.11$ & $\ldots$ & $12.61 \pm 0.24$ & -2.28 & 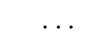 \\
\hline 111 & 4 & $16.54 \pm 0.14$ & $14.88 \pm 0.06$ & $14.23 \pm 0.07$ & $12.78 \pm 0.17$ & $12.44 \pm 0.24$ & $\ldots$ & $\ldots$ & -1.53 & $\ldots$ \\
\hline 112 & 3 & $\ldots$ & $14.93 \pm 0.08$ & $13.73 \pm 0.06$ & $12.80 \pm 0.14$ & $12.19 \pm 0.12$ & $\ldots$ & $\ldots$ & -0.50 & $\ldots$ \\
\hline 113 & 4 & $\ldots$ & $14.81 \pm 0.06$ & $\cdots$ & $12.80 \pm 0.08$ & $12.43 \pm 0.09$ & $12.14 \pm 0.13$ & $11.28 \pm 0.18$ & -1.16 & $\cdots$ \\
\hline 115 & 3 & $\ldots$ & $15.10 \pm 0.10$ & $13.80 \pm 0.08$ & $11.59 \pm 0.15$ & $11.11 \pm 0.14$ & $\ldots$ & $\ldots$ & -0.99 & \\
\hline 117 & 3 & $\ldots$ & $14.04 \pm 0.04$ & $\cdots$ & $12.06 \pm 0.08$ & $11.54 \pm 0.07$ & $11.01 \pm 0.39$ & $\cdots$ & -0.86 & \\
\hline 118 & 3 & $17.15 \pm 0.19$ & $15.92 \pm 0.14$ & $14.51 \pm 0.08$ & $13.15 \pm 0.10$ & $12.59 \pm 0.09$ & $12.16 \pm 0.14$ & $11.46 \pm 0.30$ & -0.95 & \\
\hline
\end{tabular}


Table 9 - Continued

\begin{tabular}{|c|c|c|c|c|c|c|c|c|c|c|}
\hline $\begin{array}{l}S p . \\
\text { ID }\end{array}$ & $\begin{array}{l}\text { Ep. } \\
\text { ID }\end{array}$ & $\begin{array}{c}\mathrm{J} \\
{[\mathrm{mag} .]}\end{array}$ & $\begin{array}{c}\mathrm{H} \\
{[\mathrm{mag} .]}\end{array}$ & $\begin{array}{c}\mathrm{K} \\
{[\mathrm{mag} .]}\end{array}$ & $\begin{array}{l}3.6 \mu \mathrm{m} \\
\text { [mag.] }\end{array}$ & $\begin{array}{l}4.5 \mu \mathrm{m} \\
{[\mathrm{mag} .]}\end{array}$ & $\begin{array}{l}5.8 \mu \mathrm{m} \\
\text { [mag.] }\end{array}$ & $\begin{array}{l}8.0 \mu \mathrm{m} \\
{[\mathrm{mag} .]}\end{array}$ & $\alpha_{I R A C}$ & $\begin{array}{c}A_{K} \\
{[\mathrm{mag} .]}\end{array}$ \\
\hline 119 & 3 & $12.32 \pm 0.02$ & $11.20 \pm 0.02$ & $10.62 \pm 0.02$ & $10.25 \pm 0.03$ & $10.07 \pm 0.03$ & $9.82 \pm 0.11$ & $\ldots$ & -2.18 & 0.43 \\
\hline 120 & 3 & $13.27 \pm 0.03$ & $12.71 \pm 0.03$ & $12.44 \pm 0.04$ & $12.31 \pm 0.08$ & $12.14 \pm 0.09$ & $\ldots$ & $\cdots$ & -2.20 & $\cdots$ \\
\hline 121 & 3 & $15.69 \pm 0.08$ & $14.05 \pm 0.03$ & $13.05 \pm 0.03$ & $12.16 \pm 0.09$ & $11.74 \pm 0.08$ & $11.21 \pm 0.53$ & $\ldots$ & -1.28 & 0.87 \\
\hline 122 & 1 & $16.37 \pm 0.13$ & $14.69 \pm 0.04$ & $13.80 \pm 0.05$ & $12.83 \pm 0.08$ & $12.39 \pm 0.08$ & $12.01 \pm 0.10$ & $11.59 \pm 0.14$ & -1.53 & 0.44 \\
\hline 124 & 3 & $16.77 \pm 0.19$ & $15.34 \pm 0.12$ & $14.39 \pm 0.13$ & $13.02 \pm 0.15$ & $12.60 \pm 0.15$ & 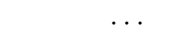 & . & -1.22 & . \\
\hline 127 & 3 & $13.83 \pm 0.03$ & $12.42 \pm 0.03$ & $11.85 \pm 0.03$ & $11.14 \pm 0.04$ & $10.79 \pm 0.04$ & $10.70 \pm 0.12$ & $10.65 \pm 0.51$ & -2.49 & 0.86 \\
\hline 128 & 3 & & & $14.61 \pm 0.12$ & & $12.26 \pm 0.14$ & & & & $\ldots$ \\
\hline 130 & 3 & $15.68 \pm 0.05$ & $14.05 \pm 0.03$ & $13.13 \pm 0.04$ & $12.13 \pm 0.06$ & $11.66 \pm 0.06$ & $11.33 \pm 0.08$ & $10.60 \pm 0.12$ & -1.30 & 0.91 \\
\hline 131 & 3 & $16.80 \pm 0.19$ & $15.65 \pm 0.14$ & $14.29 \pm 0.09$ & $13.10 \pm 0.14$ & $12.52 \pm 0.13$ & $\cdots$ & $\cdots$ & -0.60 & $\ldots$ \\
\hline 132 & 3 & $\cdots$ & & $13.19 \pm 0.11$ & $11.41 \pm 0.15$ & $10.83 \pm 0.19$ & $\cdots$ & $\cdots$ & -0.59 & $\cdots$ \\
\hline 134 & 3 & $16.83 \pm 0.20$ & $15.60 \pm 0.12$ & $14.99 \pm 0.13$ & $14.52 \pm 0.20$ & $14.29 \pm 0.25$ & $\cdots$ & $\ldots$ & -1.98 & $\cdots$ \\
\hline 135 & 3 & $15.14 \pm 0.05$ & $13.84 \pm 0.04$ & $12.97 \pm 0.04$ & $11.91 \pm 0.06$ & $11.54 \pm 0.06$ & $10.99 \pm 0.12$ & $10.08 \pm 0.25$ & -0.81 & 0.43 \\
\hline 136 & 3 & & $14.26 \pm 0.06$ & $13.46 \pm 0.07$ & $12.71 \pm 0.19$ & $12.49 \pm 0.22$ & $\cdots$ & $\cdots$ & -2.01 & $\cdots$ \\
\hline 137 & 3 & $16.88 \pm 0.20$ & $15.86 \pm 0.14$ & $15.21 \pm 0.16$ & $14.43 \pm 0.18$ & $14.29 \pm 0.20$ & $\ldots$ & $\ldots$ & -2.31 & $\ldots$ \\
\hline 139 & 3 & $\ldots$ & & $13.41 \pm 0.05$ & $12.94 \pm 0.12$ & $12.80 \pm 0.13$ & \pm 0.57 & $\ldots$ & -0.94 & $\ldots$ \\
\hline 140 & 2 & $\cdots$ & $15.54 \pm 0.15$ & $14.66 \pm 0.10$ & $13.27 \pm 0.11$ & $12.75 \pm 0.10$ & $12.44 \pm 0.20$ & $11.46 \pm 0.24$ & -0.85 & $\ldots$ \\
\hline 142 & 3 & $15.32 \pm 0.06$ & $13.98 \pm 0.04$ & $13.52 \pm 0.05$ & $13.27 \pm 0.13$ & $13.04 \pm 0.13$ & $\ldots$ & $\ldots$ & -2.25 & 0.79 \\
\hline 143 & 3 & $16.08 \pm 0.11$ & $14.51 \pm 0.06$ & $13.66 \pm 0.07$ & 11.6 & $11.31 \pm 0.14$ & $\cdots$ & $\cdots$ & -1.68 & $\cdots$ \\
\hline 145 & 3 & & $15.66 \pm 0.12$ & $14.36 \pm 0.10$ & $13.37 \pm 0.11$ & $12.88 \pm 0.10$ & $12.62 \pm 0.18$ & $11.66 \pm 0.25$ & -0.97 & $\ldots$ \\
\hline 146 & 3 & $15.87 \pm 0.09$ & $14.20 \pm 0.05$ & $13.48 \pm 0.05$ & $12.29 \pm 0.18$ & $11.86 \pm 0.13$ & $\cdots$ & $\cdots$ & -1.62 & 1.13 \\
\hline 147 & 3 & $13.07 \pm 0.05$ & $12.42 \pm 0.05$ & $12.20 \pm 0.05$ & $12.04 \pm 0.07$ & $12.00 \pm 0.09$ & $11.21 \pm 0.20$ & $\ldots$ & -1.28 & $\ldots$ \\
\hline 148 & 3 & $15.03 \pm 0.06$ & $13.59 \pm 0.04$ & $12.94 \pm 0.04$ & 12.2 & 11. & \pm 0.37 & $\cdots$ & -2.12 & 0.86 \\
\hline 149 & 3 & $16.99 \pm 0.23$ & $14.34 \pm 0.05$ & $13.11 \pm 0.04$ & $12.00 \pm 0.07$ & $11.61 \pm 0.06$ & $10.70 \pm 0.23$ & $\ldots$ & -0.77 & 1.48 \\
\hline 150 & 3 & & $15.01 \pm 0.06$ & $13.75 \pm 0.04$ & $12.66 \pm 0.11$ & $12.12 \pm 0.09$ & $11.53 \pm 0.58$ & $\cdots$ & -1.08 & 1.36 \\
\hline 151 & 1 & \pm 0.04 & $13.23 \pm 0.03$ & $12.57 \pm 0.03$ & $11.54 \pm 0.05$ & $10.99 \pm 0.04$ & $10.37 \pm 0.05$ & $9.63 \pm 0.07$ & -0.78 & 0.77 \\
\hline 152 & 3 & $12.22 \pm 0.02$ & $10.99 \pm 0.03$ & $10.32 \pm 0.02$ & $9.96 \pm 0.02$ & $9.83 \pm 0.03$ & $9.69 \pm 0.08$ & $9.13 \pm 0.21$ & -2.03 & 0.50 \\
\hline 154 & 2 & $13.97 \pm 0.03$ & $13.13 \pm 0.03$ & $12.68 \pm 0.03$ & $12.05 \pm 0.06$ & $11.80 \pm 0.06$ & $11.60 \pm 0.11$ & $10.72 \pm 0.24$ & -1.40 & 0.09 \\
\hline 155 & 3 & & $15.56 \pm 0.12$ & $14.56 \pm 0.11$ & $13.86 \pm 0.18$ & $13.78 \pm 0.19$ & $\cdots$ & $\cdots$ & -2.56 & $\cdots$ \\
\hline 157 & 3 & $15.05 \pm 0.04$ & $12.93 \pm 0.04$ & $11.74 \pm 0.03$ & $10.20 \pm 0.06$ & $9.73 \pm 0.06$ & $8.92 \pm 0.23$ & $\ldots$ & -0.79 & 1.43 \\
\hline 158 & 3 & $14.44 \pm 0.03$ & $11.18 \pm 0.02$ & $9.41 \pm 0.03$ & $8.27 \pm 0.01$ & $7.92 \pm 0.01$ & $7.63 \pm 0.03$ & $7.14 \pm 0.09$ & -2.02 & 2.73 \\
\hline 159 & 3 & $11.89 \pm 0.02$ & $11.50 \pm 0.03$ & $11.43 \pm 0.02$ & $11.43 \pm 0.06$ & $11.46 \pm 0.07$ & $11.20 \pm 0.58$ & $\cdots$ & -2.44 & $\cdots$ \\
\hline 160 & 3 & $16.67 \pm 0.16$ & $15.53 \pm 0.13$ & $14.97 \pm 0.15$ & $13.65 \pm 0.17$ & $13.58 \pm 0.19$ & $\ldots$ & $\ldots$ & -2.57 & $\ldots$ \\
\hline 161 & 2 & $15.89 \pm 0.07$ & $14.69 \pm 0.07$ & $13.90 \pm 0.07$ & $\cdots$ & $12.73 \pm 0.10$ & $\cdots$ & $11.35 \pm 0.47$ & -0.64 & 0.35 \\
\hline 162 & 3 & $11.36 \pm 0.02$ & $11.01 \pm 0.03$ & $10.88 \pm 0.02$ & $10.91 \pm 0.04$ & $10.92 \pm 0.04$ & $10.55 \pm 0.13$ & $\ldots$ & -2.19 & $\ldots$ \\
\hline 163 & 1 & $\cdots$ & $\cdots$ & $14.97 \pm 0.13$ & $13.60 \pm 0.12$ & $12.93 \pm 0.11$ & $12.39 \pm 0.14$ & $11.59 \pm 0.19$ & -0.58 & $\cdots$ \\
\hline 167 & 4 & $\ldots$ & $15.94 \pm 0.14$ & $15.38 \pm 0.19$ & $14.04 \pm 0.15$ & $13.65 \pm 0.15$ & $\ldots$ & $\cdots$ & -1.34 & $\ldots$ \\
\hline 168 & 4 & $\cdots$ & $\cdots$ & $14.93 \pm 0.14$ & $12.94 \pm 0.16$ & $\cdots$ & $\cdots$ & $\cdots$ & $\cdots$ & $\cdots$ \\
\hline 170 & 3 & $\ldots$ & $15.75 \pm 0.13$ & $14.69 \pm 0.11$ & $13.80 \pm 0.20$ & $13.67 \pm 0.20$ & $\cdots$ & . & -2.32 & $\ldots$ \\
\hline 171 & 3 & $12.48 \pm 0.02$ & $10.95 \pm 0.02$ & $10.31 \pm 0.02$ & $9.89 \pm 0.04$ & $9.81 \pm 0.04$ & $\ldots$ & $\cdots$ & -2.94 & 0.98 \\
\hline 172 & 3 & $17.04 \pm 0.21$ & $15.24 \pm 0.08$ & $14.22 \pm 0.08$ & $13.13 \pm 0.11$ & $12.74 \pm 0.10$ & $\cdots$ & $\cdots$ & -1.35 & $\cdots$ \\
\hline 175 & 3 & $\ldots$ & $15.76 \pm 0.14$ & $15.20 \pm 0.16$ & $13.11 \pm 0.13$ & $12.81 \pm 0.18$ & $\ldots$ & $\cdots$ & -1.71 & $\ldots$ \\
\hline 176 & 3 & $\cdots$ & $15.02 \pm 0.07$ & $13.85 \pm 0.04$ & $12.56 \pm 0.10$ & $12.01 \pm 0.08$ & $11.61 \pm 0.62$ & $\cdots$ & -1.28 & 0.78 \\
\hline 177 & 4 & $16.89 \pm 0.21$ & $15.40 \pm 0.10$ & $14.82 \pm 0.12$ & $13.45 \pm 0.14$ & $13.32 \pm 0.20$ & $\ldots$ & $\ldots$ & -2.37 & $\ldots$ \\
\hline 178 & 3 & $13.21 \pm 0.03$ & $12.38 \pm 0.03$ & $11.90 \pm 0.03$ & $11.64 \pm 0.07$ & $11.43 \pm 0.07$ & $\cdots$ & $\cdots$ & -2.04 & 0.04 \\
\hline 179 & 4 & $16.85 \pm 0.14$ & $15.13 \pm 0.09$ & $14.16 \pm 0.07$ & $12.80 \pm 0.08$ & $12.30 \pm 0.08$ & $12.07 \pm 0.12$ & $11.65 \pm 0.25$ & -1.67 & 0.47 \\
\hline
\end{tabular}


Table 9 - Continued

\begin{tabular}{|c|c|c|c|c|c|c|c|c|c|c|}
\hline $\begin{array}{l}S p . \\
\text { ID }\end{array}$ & $\begin{array}{l}\text { Ep. } \\
\text { ID }\end{array}$ & $\begin{array}{c}\mathrm{J} \\
{[\mathrm{mag} .]}\end{array}$ & $\begin{array}{c}\mathrm{H} \\
{[\mathrm{mag} .]}\end{array}$ & $\begin{array}{c}\mathrm{K} \\
{[\mathrm{mag} .]}\end{array}$ & $\begin{array}{l}3.6 \mu \mathrm{m} \\
\text { [mag.] }\end{array}$ & $\begin{array}{l}4.5 \mu \mathrm{m} \\
{[\mathrm{mag} .]}\end{array}$ & $\begin{array}{l}5.8 \mu \mathrm{m} \\
\text { [mag.] }\end{array}$ & $\begin{array}{l}8.0 \mu \mathrm{m} \\
{[\mathrm{mag} .]}\end{array}$ & $\alpha_{I R A C}$ & $\begin{array}{c}A_{K} \\
{[\mathrm{mag} .]}\end{array}$ \\
\hline 180 & 1 & $15.18 \pm 0.05$ & $14.00 \pm 0.03$ & $13.39 \pm 0.04$ & $12.86 \pm 0.09$ & $12.60 \pm 0.09$ & $12.39 \pm 0.12$ & $11.72 \pm 0.13$ & -1.68 & 0.49 \\
\hline 184 & 1 & $15.67 \pm 0.08$ & $13.96 \pm 0.11$ & $12.87 \pm 0.06$ & $11.54 \pm 0.19$ & $11.07 \pm 0.16$ & $\cdots$ & $\cdots$ & -1.02 & $\cdots$ \\
\hline 186 & 3 & $14.13 \pm 0.04$ & $13.03 \pm 0.04$ & $12.52 \pm 0.05$ & $11.47 \pm 0.06$ & $11.28 \pm 0.07$ & $\cdots$ & $\cdots$ & -2.29 & 0.45 \\
\hline 188 & 3 & $16.81 \pm 0.18$ & $15.61 \pm 0.13$ & $14.77 \pm 0.11$ & $13.52 \pm 0.14$ & $13.00 \pm 0.12$ & $\cdots$ & $\cdots$ & -0.83 & $\cdots$ \\
\hline 189 & 2 & & $15.98 \pm 0.15$ & $14.90 \pm 0.14$ & $14.10 \pm 0.16$ & $13.96 \pm 0.18$ & $\cdots$ & $\ldots$ & -2.31 & . \\
\hline 191 & 4 & $=15.79 \pm 0.08$ & $14.42 \pm 0.06$ & $13.63 \pm 0.06$ & $12.67 \pm 0.08$ & $12.19 \pm 0.08$ & $11.65 \pm 0.10$ & $10.64 \pm 0.11$ & -0.63 & 0.61 \\
\hline 192 & 4 & $16.66 \pm 0.17$ & $14.89 \pm 0.07$ & $14.23 \pm 0.06$ & $13.14 \pm 0.12$ & $12.78 \pm 0.12$ & $12.97 \pm 1.09$ & $\ldots$ & -2.56 & $\ldots$ \\
\hline 196 & 4 & & $15.06 \pm 0.09$ & $13.84 \pm 0.06$ & $12.52 \pm 0.08$ & $12.00 \pm 0.07$ & $12.15 \pm 0.44$ & $\cdots$ & -2.46 & 1.03 \\
\hline 197 & 3 & $15.92 \pm 0.10$ & $13.93 \pm 0.04$ & $12.62 \pm 0.03$ & $11.09 \pm 0.05$ & $10.53 \pm 0.04$ & $10.29 \pm 0.26$ & $\ldots$ & 65 & 1.13 \\
\hline 199 & 3 & $16.74 \pm 0.17$ & $14.91 \pm 0.06$ & $13.88 \pm 0.05$ & $12.80 \pm 0.08$ & $12.42 \pm 0.08$ & $12.18 \pm 0.14$ & $11.48 \pm 0.38$ & -1.57 & 1.02 \\
\hline 200 & 3 & $15.54 \pm 0.09$ & $13.09 \pm 0.03$ & $12.14 \pm 0.03$ & $10.92 \pm 0.14$ & $10.34 \pm 0.26$ & $\cdots$ & $\ldots$ & -1.36 & 1.95 \\
\hline 202 & 3 & $17.08 \pm 0.23$ & $15.11 \pm 0.09$ & $14.70 \pm 0.12$ & $14.56 \pm 0.21$ & $14.42 \pm 0.22$ & $\ldots$ & $\ldots$ & -2.28 & $\ldots$ \\
\hline 204 & 1 & $\cdots$ & $15.84 \pm 0.14$ & $14.77 \pm 0.12$ & & $12.50 \pm 0.15$ & $\cdots$ & $\cdots$ & & $\cdots$ \\
\hline 205 & 3 & $15.42 \pm 0.05$ & $13.89 \pm 0.03$ & $12.93 \pm 0.03$ & $12.13 \pm 0.08$ & $11.78 \pm 0.07$ & $11.88 \pm 0.77$ & $\ldots$ & -2.60 & 0.72 \\
\hline 206 & 2 & $16.97 \pm 0.20$ & $15.76 \pm 0.14$ & $14.94 \pm 0.14$ & & $13.70 \pm 0.15$ & $\ldots$ & $13.09 \pm 0.34$ & -1.91 & $\ldots$ \\
\hline 209 & 3 & $14.75 \pm 0.03$ & $13.26 \pm 0.03$ & $12.63 \pm 0.03$ & $12.08 \pm 0.07$ & $11.96 \pm 0.08$ & $11.24 \pm 0.27$ & $\ldots$ & -1.50 & 0.93 \\
\hline 211 & 3 & $15.10 \pm 0.05$ & $13.69 \pm 0.04$ & $12.81 \pm 0.04$ & $11.42 \pm 0.05$ & $10.92 \pm 0.04$ & $10.22 \pm 0.07$ & $\cdots$ & -0.74 & 0.61 \\
\hline 212 & 3 & $16.85 \pm 0.20$ & $15.38 \pm 0.10$ & $14.67 \pm 0.13$ & $13.07 \pm 0.17$ & $12.99 \pm 0.18$ & $\ldots$ & $\cdots$ & -2.55 & $\cdots$ \\
\hline 213 & 3 & & $16.37 \pm 0.24$ & $14.81 \pm 0.16$ & $11.64 \pm 0.14$ & $11.19 \pm 0.17$ & $\cdots$ & $\ldots$ & -1.11 & $\cdots$ \\
\hline 216 & 3 & $14.97 \pm 0.03$ & $13.31 \pm 0.02$ & $12.31 \pm 0.02$ & $11.38 \pm 0.04$ & $11.01 \pm 0.04$ & $10.80 \pm 0.08$ & \pm 0.25 & -2.14 & 0.88 \\
\hline 217 & 4 & $=16.26 \pm 0.13$ & $14.36 \pm 0.24$ & $13.42 \pm 0.05$ & $12.15 \pm 0.07$ & $11.64 \pm 0.07$ & $10.96 \pm 0.19$ & $\ldots$ & -0.59 & 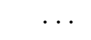 \\
\hline 218 & 3 & $17.18 \pm 0.23$ & $14.89 \pm 0.06$ & $13.87 \pm 0.06$ & $12.97 \pm 0.16$ & $12.88 \pm 0.20$ & $\ldots$ & $\cdots$ & -2.51 & $\cdots$ \\
\hline 219 & 1 & $14.04 \pm 0.02$ & $13.56 \pm 0.03$ & $13.38 \pm 0.05$ & $\ldots$ & $11.87 \pm 0.09$ & $\ldots$ & $\ldots$ & & $\ldots$ \\
\hline 222 & 3 & $15.77 \pm 0.06$ & $14.04 \pm 0.04$ & $13.24 \pm 0.04$ & $12.24 \pm 0.07$ & $11.77 \pm 0.06$ & $11.44 \pm 0.12$ & $10.57 \pm 0.22$ & -1.18 & 1.18 \\
\hline 223 & 3 & & $15.72 \pm 0.14$ & $14.41 \pm 0.11$ & $12.79 \pm 0.19$ & $12.29 \pm 0.14$ & $\ldots$ & $\ldots$ & -0.90 & $\ldots$ \\
\hline 224 & 4 & \pm 0.25 & $16.22 \pm 0.18$ & $15.21 \pm 0.16$ & $\cdots$ & $13.16 \pm 0.12$ & $\cdots$ & $12.33 \pm 0.18$ & -1 & $\cdots$ \\
\hline 226 & 4 & $=14.70 \pm 0.08$ & $13.39 \pm 0.06$ & $12.83 \pm 0.05$ & . & 11.58 & $\ldots$ & $10.24 \pm 0.07$ & -0.75 & 0.75 \\
\hline 228 & 3 & $17.05 \pm 0.21$ & $15.34 \pm 0.09$ & $14.06 \pm 0.06$ & $13.21 \pm 0.29$ & $12.84 \pm 0.22$ & $\ldots$ & $\cdots$ & -1.40 & $\cdots$ \\
\hline 231 & 4 & $\cdots$ & $15.24 \pm 0.08$ & $14.34 \pm 0.09$ & & $12.67 \pm 0.09$ & $\cdots$ & $11.46 \pm 0.19$ & -0.92 & 0.21 \\
\hline 232 & 4 & . & $15.44 \pm 0.13$ & $14.58 \pm 0.11$ & . & $12.85 \pm 0.10$ & $\ldots$ & $11.69 \pm 0.13$ & -0 & $\cdots$ \\
\hline 233 & 3 & $13.35 \pm 0.03$ & $12.10 \pm 0.02$ & $11.41 \pm 0.02$ & $10.43 \pm 0.03$ & $10.05 \pm 0.03$ & $9.73 \pm 0.04$ & $9.37 \pm 0.08$ & -1.76 & 0.51 \\
\hline 234 & 1 & $15.54 \pm 0.06$ & $13.88 \pm 0.05$ & $13.08 \pm 0.04$ & $11.93 \pm 0.06$ & $11.45 \pm 0.05$ & $11.07 \pm 0.07$ & $10.53 \pm 0.13$ & -1.46 & 1.07 \\
\hline 235 & 1 & $\cdots$ & $15.08 \pm 0.07$ & $13.80 \pm 0.07$ & $\cdots$ & $11.88 \pm 0.08$ & $\cdots$ & $\cdots$ & $\cdots$ & 1.03 \\
\hline 236 & 3 & \pm 0.04 & $12.08 \pm 0.03$ & $10.60 \pm 0.02$ & $8.69 \pm 0.01$ & $8.03 \pm 0.01$ & \pm 0.01 & $=0.03$ & -0 & 1.48 \\
\hline 237 & 1 & $15.51 \pm 0.08$ & $14.18 \pm 0.06$ & $13.49 \pm 0.06$ & $12.63 \pm 0.08$ & $12.20 \pm 0.08$ & $11.67 \pm 0.10$ & $10.63 \pm 0.14$ & -0.67 & 0.66 \\
\hline 238 & 3 & & $14.42 \pm 0.06$ & $13.30 \pm 0.07$ & $11.11 \pm 0.16$ & $10.63 \pm 0.17$ & $\ldots$ & $\ldots$ & -0.99 & $\ldots$ \\
\hline 241 & 3 & $15.60 \pm 0.06$ & $14.94 \pm 0.06$ & $14.26 \pm 0.09$ & $13.24 \pm 0.13$ & $12.96 \pm 0.13$ & $\cdots$ & $\cdots$ & -1.77 & 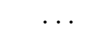 \\
\hline 242 & 4 & $=16.04 \pm 0.08$ & $14.41 \pm 0.04$ & $13.46 \pm 0.03$ & $12.61 \pm 0.19$ & $12.22 \pm 0.12$ & $\cdots$ & $\ldots$ & -1.67 & 0.87 \\
\hline 244 & 3 & $10.62 \pm 0.02$ & $9.29 \pm 0.03$ & $8.48 \pm 0.02$ & $7.82 \pm 0.01$ & $7.54 \pm 0.01$ & $7.27 \pm 0.02$ & $6.74 \pm 0.06$ & -1.73 & 0.55 \\
\hline 245 & 1 & $15.07 \pm 0.05$ & $13.77 \pm 0.05$ & $13.22 \pm 0.04$ & $12.56 \pm 0.07$ & $12.14 \pm 0.07$ & $11.73 \pm 0.08$ & $10.91 \pm 0.16$ & -1.10 & 0.71 \\
\hline 246 & 3 & $13.76 \pm 0.03$ & $12.73 \pm 0.03$ & $12.35 \pm 0.03$ & $12.17 \pm 0.06$ & $12.12 \pm 0.07$ & $11.94 \pm 0.24$ & $\cdots$ & -2.56 & 0.44 \\
\hline 247 & 3 & & $14.83 \pm 0.07$ & $12.67 \pm 0.03$ & $\cdots$ & $9.49 \pm 0.02$ & $\cdots$ & $8.01 \pm 0.07$ & -0.58 & 1.95 \\
\hline 248 & 4 & $14.70 \pm 0.04$ & $13.46 \pm 0.05$ & $12.80 \pm 0.03$ & & $11.49 \pm 0.05$ & $\ldots$ & $10.93 \pm 0.09$ & -2.01 & 0.52 \\
\hline 249 & 3 & $13.65 \pm 0.12$ & $12.24 \pm 0.06$ & $11.04 \pm 0.05$ & $9.57 \pm 0.05$ & $8.93 \pm 0.06$ & $8.56 \pm 0.11$ & $\cdots$ & -1.09 & 0.57 \\
\hline 250 & 1 & $15.39 \pm 0.06$ & $14.13 \pm 0.04$ & $13.42 \pm 0.04$ & $12.64 \pm 0.08$ & $12.23 \pm 0.08$ & $11.84 \pm 0.08$ & $11.29 \pm 0.12$ & -1.42 & 0.53 \\
\hline
\end{tabular}


Table 9 - Continued

\begin{tabular}{|c|c|c|c|c|c|c|c|c|c|c|}
\hline $\begin{array}{l}S p . \\
\text { ID }\end{array}$ & $\begin{array}{l}\text { Ep. } \\
\text { ID }\end{array}$ & $\begin{array}{c}\mathrm{J} \\
{[\mathrm{mag} .]}\end{array}$ & $\begin{array}{c}\mathrm{H} \\
{[\mathrm{mag} .]}\end{array}$ & $\begin{array}{c}\mathrm{K} \\
{[\mathrm{mag} .]}\end{array}$ & $\begin{array}{l}3.6 \mu \mathrm{m} \\
\text { [mag.] }\end{array}$ & $\begin{array}{l}4.5 \mu \mathrm{m} \\
{[\mathrm{mag} .]}\end{array}$ & $\begin{array}{l}5.8 \mu \mathrm{m} \\
\text { [mag.] }\end{array}$ & $\begin{array}{l}8.0 \mu \mathrm{m} \\
{[\mathrm{mag} .]}\end{array}$ & $\alpha_{I R A C}$ & $\begin{array}{c}A_{K} \\
{[\mathrm{mag} .]}\end{array}$ \\
\hline 251 & 3 & $\ldots$ & $14.59 \pm 0.08$ & $13.03 \pm 0.07$ & $11.74 \pm 0.28$ & $11.19 \pm 0.27$ & $\ldots$ & $\ldots$ & -0.70 & \\
\hline 252 & 3 & $\ldots$ & $15.89 \pm 0.14$ & $15.30 \pm 0.20$ & $14.89 \pm 0.27$ & $14.90 \pm 0.39$ & $\ldots$ & $\cdots$ & -2.89 & $\cdots$ \\
\hline 253 & 4 & $16.91 \pm 0.20$ & $15.51 \pm 0.13$ & $14.53 \pm 0.12$ & $13.81 \pm 0.24$ & $13.24 \pm 0.17$ & $\ldots$ & $\ldots$ & -0.66 & $\ldots$ \\
\hline 254 & 3 & $16.63 \pm 0.15$ & $14.78 \pm 0.05$ & $13.74 \pm 0.04$ & $12.49 \pm 0.08$ & $12.04 \pm 0.08$ & $11.21 \pm 0.11$ & $\cdots$ & -0.62 & 0.79 \\
\hline 256 & 3 & $\ldots$ & $15.81 \pm 0.14$ & $13.97 \pm 0.09$ & $12.20 \pm 0.07$ & $11.45 \pm 0.05$ & $11.06 \pm 0.09$ & $10.15 \pm 0.15$ & -0.59 & $\ldots$ \\
\hline 257 & 3 & $\cdots$ & $14.58 \pm 0.05$ & $13.65 \pm 0.05$ & $11.62 \pm 0.16$ & $11.48 \pm 0.25$ & $\ldots$ & $\ldots$ & -2.31 & $\ldots$ \\
\hline 259 & 3 & & $14.52 \pm 0.08$ & $13.02 \pm 0.07$ & $11.27 \pm 0.10$ & $10.72 \pm 0.08$ & $\ldots$ & $\ldots$ & -1.17 & 1.17 \\
\hline 261 & 2 & $17.36 \pm 0.30$ & $15.10 \pm 0.08$ & $13.70 \pm 0.05$ & & $11.53 \pm 0.20$ & $\cdots$ & $\cdots$ & & $\ldots$ \\
\hline 264 & 3 & $15.51 \pm 0.06$ & $14.52 \pm 0.06$ & $13.96 \pm 0.06$ & $13.73 \pm 0.14$ & $13.58 \pm 0.14$ & $\cdots$ & $\cdots$ & -2.36 & 0.23 \\
\hline 265 & 3 & $\ldots$ & $16.05 \pm 0.18$ & $15.15 \pm 0.13$ & $13.65 \pm 0.13$ & $13.01 \pm 0.11$ & $\cdots$ & $\cdots$ & -0.35 & $\cdots$ \\
\hline 267 & 1 & $17.10 \pm 0.23$ & $15.01 \pm 0.09$ & $13.61 \pm 0.05$ & $11.58 \pm 0.11$ & $11.08 \pm 0.09$ & $\cdots$ & $\cdots$ & -1.13 & 0.54 \\
\hline 268 & 3 & $15.92 \pm 0.09$ & $15.47 \pm 0.10$ & $14.79 \pm 0.11$ & $15.11 \pm 0.25$ & $14.96 \pm 0.27$ & $\ldots$ & $\ldots$ & -2.29 & $\ldots$ \\
\hline 269 & 4 & $15.92 \pm 0.07$ & $13.88 \pm 0.03$ & $12.50 \pm 0.02$ & $11.14 \pm 0.05$ & $10.54 \pm 0.04$ & $10.37 \pm 0.20$ & $10.69 \pm 1.43$ & -2.58 & 1.15 \\
\hline 272 & 4 & $13.27 \pm 0.03$ & $12.08 \pm 0.02$ & $11.32 \pm 0.02$ & $\ldots$ & $9.80 \pm 0.02$ & $\ldots$ & $8.24 \pm 0.03$ & -0.35 & 0.36 \\
\hline 273 & 3 & & $15.04 \pm 0.07$ & $13.94 \pm 0.07$ & $13.21 \pm 0.13$ & $12.91 \pm 0.12$ & $\cdots$ & $\ldots$ & -1.72 & $\cdots$ \\
\hline 275 & 1 & $16.86 \pm 0.18$ & $15.52 \pm 0.13$ & $14.36 \pm 0.09$ & $13.13 \pm 0.10$ & $12.52 \pm 0.09$ & $12.00 \pm 0.11$ & $11.08 \pm 0.13$ & -0 & $\ldots$ \\
\hline 276 & 4 & $16.58 \pm 0.15$ & $15.29 \pm 0.09$ & $14.29 \pm 0.07$ & & $12.86 \pm 0.10$ & $\ldots$ & $11.33 \pm 0.16$ & 37 & $\ldots$ \\
\hline 277 & 1 & $15.65 \pm 0.08$ & $14.40 \pm 0.07$ & $13.82 \pm 0.05$ & $12.95 \pm 0.09$ & $12.53 \pm 0.09$ & $12.11 \pm 0.12$ & $11.30 \pm 0.23$ & -1.09 & 0.62 \\
\hline 279 & 2 & & $15.78 \pm 0.13$ & $13.81 \pm 0.06$ & $12.13 \pm 0.06$ & $11.47 \pm 0.05$ & $11.04 \pm 0.10$ & $10.36 \pm 0.22$ & -0.89 & $\ldots$ \\
\hline 282 & 3 & $13.30 \pm 0.03$ & $12.58 \pm 0.04$ & $12.25 \pm 0.06$ & $12.40 \pm 0.13$ & $12.22 \pm 0.19$ & $\cdots$ & $\cdots$ & -2.14 & $\cdots$ \\
\hline 283 & 4 & $14.82 \pm 0.04$ & $13.46 \pm 0.03$ & $12.84 \pm 0.03$ & $12.17 \pm 0.06$ & $11.85 \pm 0.06$ & $11.48 \pm 0.08$ & $11.05 \pm 0.17$ & -1.70 & 0.77 \\
\hline 284 & 1 & $16.21 \pm 0.11$ & $14.75 \pm 0.06$ & $13.77 \pm 0.05$ & $12.64 \pm 0.08$ & 12.21 & $11.92 \pm 0.11$ & $11.06 \pm 0.20$ & -1.21 & 0.71 \\
\hline 285 & 3 & & $15.50 \pm 0.10$ & (2) & $14.56 \pm 0.21$ & $14.47 \pm 0.23$ & $\ldots$ & $\ldots$ & -2.51 & $\ldots$ \\
\hline 286 & 1 & $15.17 \pm 0.04$ & $13.52 \pm 0.03$ & $12.59 \pm 0.03$ & $11.67 \pm 0.05$ & $11.19 \pm 0.05$ & $10.93 \pm 0.09$ & $10.04 \pm 0.18$ & -1.20 & 0.93 \\
\hline 287 & 3 & $16.29 \pm 0.14$ & $15.41 \pm 0.10$ & $14.65 \pm 0.11$ & $14.11 \pm 0.15$ & $13.75 \pm 0.16$ & $\ldots$ & & -1.45 & $\ldots$ \\
\hline 288 & 1 & $11.38 \pm 0.02$ & $10.81 \pm 0.02$ & $10.43 \pm 0.02$ & $10.04 \pm 0.02$ & $9.59 \pm 0.02$ & $9.44 \pm 0.03$ & $9.30 \pm 0.08$ & -2.09 & $\cdots$ \\
\hline 289 & 1 & $15.19 \pm 0.05$ & $13.58 \pm 0.02$ & $12.76 \pm 0.03$ & $11.41 \pm 0.04$ & $10.74 \pm 0.04$ & $10.11 \pm 0.04$ & $9.23 \pm 0.05$ & -0.52 & 0.96 \\
\hline 293 & 3 & $11.70 \pm 0.02$ & $10.97 \pm 0.03$ & $10.50 \pm 0.03$ & $10.11 \pm 0.06$ & $9.78 \pm 0.10$ & $9.25 \pm 0.22$ & $\cdots$ & -1.22 & $\cdots$ \\
\hline 294 & 1 & $16.74 \pm 0.16$ & $15.36 \pm 0.10$ & $15.01 \pm 0.16$ & $13.91 \pm 0.14$ & $13.50 \pm 0.14$ & $13.07 \pm 0.17$ & $13.09 \pm 0.61$ & -1.91 & $\cdots$ \\
\hline 296 & 1 & $15.42 \pm 0.06$ & $14.13 \pm 0.03$ & $13.58 \pm 0.05$ & $13.05 \pm 0.09$ & $12.71 \pm 0.09$ & $12.27 \pm 0.11$ & $11.35 \pm 0.15$ & -1.03 & 0.72 \\
\hline 298 & 1 & $9.63 \pm 0.07$ & $8.86 \pm 0.12$ & $8.46 \pm 0.06$ & $9.10 \pm 0.02$ & $8.84 \pm 0.02$ & $8.74 \pm 0.02$ & $8.23 \pm 0.03$ & -1.93 & $\cdots$ \\
\hline 299 & 4 & $15.96 \pm 0.09$ & $14.64 \pm 0.05$ & $13.80 \pm 0.05$ & $\ldots$ & $12.58 \pm 0.09$ & $\ldots$ & $12.20 \pm 0.54$ & -2.31 & 0.49 \\
\hline 300 & 4 & $16.49 \pm 0.15$ & $15.30 \pm 0.09$ & $14.80 \pm 0.12$ & $\cdots$ & $13.44 \pm 0.13$ & $\cdots$ & $12.64 \pm 0.36$ & -1.58 & $\cdots$ \\
\hline 301 & 1 & $14.48 \pm 0.04$ & $13.28 \pm 0.06$ & $12.73 \pm 0.04$ & $12.46 \pm 0.07$ & $12.20 \pm 0.08$ & $11.71 \pm 0.09$ & $10.85 \pm 0.16$ & -1.08 & 0.58 \\
\hline 303 & 3 & $\cdots$ & $15.14 \pm 0.08$ & $13.72 \pm 0.05$ & $11.92 \pm 0.06$ & $11.36 \pm 0.05$ & $10.60 \pm 0.12$ & $\cdots$ & -0.74 & 1.44 \\
\hline 304 & 1 & $12.08 \pm 0.07$ & $10.73 \pm 0.09$ & $9.72 \pm 0.06$ & $8.87 \pm 0.07$ & $8.13 \pm 0.05$ & $\ldots$ & $\ldots$ & -0.14 & 0.38 \\
\hline 305 & 4 & $17.18 \pm 0.30$ & $15.39 \pm 0.11$ & $14.89 \pm 0.12$ & $13.81 \pm 0.16$ & $\cdots$ & $\cdots$ & $\cdots$ & $\cdots$ & $\cdots$ \\
\hline 307 & 3 & $\cdots$ & & $14.47 \pm 0.13$ & $10.66 \pm 0.09$ & $10.28 \pm 0.13$ & $\cdots$ & $\ldots$ & -1.39 & $\ldots$ \\
\hline 308 & 3 & $12.57 \pm 0.03$ & $10.73 \pm 0.03$ & $9.77 \pm 0.02$ & $9.20 \pm 0.02$ & $9.02 \pm 0.02$ & $8.94 \pm 0.02$ & $8.41 \pm 0.05$ & -2.20 & 1.21 \\
\hline 309 & 4 & $16.82 \pm 0.17$ & $15.45 \pm 0.11$ & $14.53 \pm 0.10$ & & $13.15 \pm 0.12$ & $\cdots$ & $12.05 \pm 0.28$ & -1.08 & $\cdots$ \\
\hline 310 & 3 & $16.01 \pm 0.09$ & $14.57 \pm 0.07$ & $13.85 \pm 0.06$ & $12.68 \pm 0.12$ & $\ldots$ & $\cdots$ & $\ldots$ & $\ldots$ & 0.79 \\
\hline 311 & 3 & $14.94 \pm 0.04$ & $12.99 \pm 0.02$ & $11.83 \pm 0.02$ & $10.80 \pm 0.03$ & $10.33 \pm 0.03$ & $9.96 \pm 0.07$ & $9.56 \pm 0.20$ & -1.64 & 1.19 \\
\hline 312 & 3 & $15.78 \pm 0.08$ & $14.59 \pm 0.06$ & $14.03 \pm 0.06$ & $13.57 \pm 0.13$ & $13.49 \pm 0.14$ & $\ldots$ & $\ldots$ & -2.78 & 0.54 \\
\hline 313 & 3 & $\cdots$ & $15.14 \pm 0.07$ & $14.15 \pm 0.06$ & $13.45 \pm 0.12$ & $13.23 \pm 0.12$ & $13.16 \pm 0.37$ & $\cdots$ & -2.32 & \\
\hline 315 & 3 & $16.84 \pm 0.20$ & $15.30 \pm 0.09$ & $14.79 \pm 0.13$ & $13.77 \pm 0.19$ & $13.53 \pm 0.17$ & . & . & -1.92 & \\
\hline
\end{tabular}


Table 9 - Continued

\begin{tabular}{|c|c|c|c|c|c|c|c|c|c|c|}
\hline $\begin{array}{l}S p . \\
\text { ID }\end{array}$ & $\begin{array}{l}\text { Ep. } \\
\text { ID }\end{array}$ & $\begin{array}{c}\mathrm{J} \\
{[\mathrm{mag} .]}\end{array}$ & $\begin{array}{c}\mathrm{H} \\
{[\mathrm{mag} .]}\end{array}$ & $\begin{array}{c}\mathrm{K} \\
{[\mathrm{mag} .]}\end{array}$ & $\begin{array}{l}3.6 \mu \mathrm{m} \\
\text { [mag.] }\end{array}$ & $\begin{array}{l}4.5 \mu \mathrm{m} \\
\text { [mag.] }\end{array}$ & $\begin{array}{l}5.8 \mu \mathrm{m} \\
\text { [mag.] }\end{array}$ & $\begin{array}{l}8.0 \mu \mathrm{m} \\
{[\mathrm{mag} .]}\end{array}$ & $\alpha_{I R A C}$ & $\begin{array}{c}A_{K} \\
{[\mathrm{mag} .]}\end{array}$ \\
\hline 319 & 1 & $17.38 \pm 0.27$ & $16.04 \pm 0.15$ & $15.44 \pm 0.19$ & $13.97 \pm 0.17$ & $13.50 \pm 0.16$ & $\cdots$ & $\ldots$ & -1.00 & \\
\hline 321 & 1 & $16.68 \pm 0.19$ & $14.85 \pm 0.09$ & $13.67 \pm 0.05$ & $12.15 \pm 0.06$ & $11.60 \pm 0.06$ & $11.12 \pm 0.07$ & $10.30 \pm 0.12$ & -0.90 & 0.85 \\
\hline 322 & 3 & $14.59 \pm 0.05$ & $13.95 \pm 0.04$ & $13.64 \pm 0.07$ & $12.77 \pm 0.16$ & $12.66 \pm 0.14$ & $\ldots$ & $\ldots$ & -2.46 & $\ldots$ \\
\hline 323 & 3 & $16.94 \pm 0.19$ & $15.69 \pm 0.13$ & $15.03 \pm 0.14$ & $13.81 \pm 0.14$ & $13.44 \pm 0.14$ & $\cdots$ & $\cdots$ & -1.41 & $\cdots$ \\
\hline 326 & 1 & & $15.91 \pm 0.14$ & $14.52 \pm 0.09$ & . & $12.43 \pm 0.10$ & $\cdots$ & $\ldots$ & . & $\ldots$ \\
\hline 328 & 3 & $14.93 \pm 0.05$ & $12.51 \pm 0.03$ & $10.84 \pm 0.02$ & $8.74 \pm 0.01$ & $8.14 \pm 0.01$ & $7.50 \pm 0.02$ & $6.69 \pm 0.06$ & -0.73 & 1.50 \\
\hline 329 & 4 & $=14.95 \pm 0.05$ & $13.63 \pm 0.04$ & $12.86 \pm 0.04$ & & $11.68 \pm 0.06$ & & $10.87 \pm 0.13$ & -1.61 & 0.57 \\
\hline 330 & 3 & $\cdots$ & $15.31 \pm 0.11$ & $13.81 \pm 0.07$ & $12.11 \pm 0.06$ & $11.38 \pm 0.05$ & $11.08 \pm 0.17$ & $\ldots$ & -0.91 & . \\
\hline 331 & 4 & $\cdots$ & & $13.11 \pm 0.04$ & $11.77 \pm 0.05$ & $11.32 \pm 0.05$ & $10.95 \pm 0.09$ & $9.99 \pm 0.17$ & -0.86 & $\ldots$ \\
\hline 332 & 3 & $15.36 \pm 0.11$ & $13.64 \pm 0.05$ & $13.10 \pm 0.05$ & $11.24 \pm 0.14$ & $10.86 \pm 0.12$ & $\ldots$ & $\cdots$ & -1.37 & $\cdots$ \\
\hline 333 & 3 & & $14.84 \pm 0.07$ & $13.98 \pm 0.08$ & $13.55 \pm 0.27$ & $13.34 \pm 0.33$ & $\cdots$ & $\cdots$ & -2.06 & $\cdots$ \\
\hline 335 & 3 & $16.24 \pm 0.10$ & $14.86 \pm 0.07$ & $14.28 \pm 0.09$ & $\ldots$ & $12.67 \pm 0.10$ & $\ldots$ & $\ldots$ & $\ldots$ & $\ldots$ \\
\hline 336 & 4 & $=16.09 \pm 0.09$ & $14.93 \pm 0.09$ & $14.06 \pm 0.07$ & $\cdots$ & $12.00 \pm 0.07$ & $\cdots$ & $10.53 \pm 0.18$ & -0.49 & 0.21 \\
\hline 338 & 3 & $\ldots$ & $15.16 \pm 0.10$ & $13.84 \pm 0.08$ & $12.14 \pm 0.11$ & $11.84 \pm 0.09$ & $\ldots$ & $\ldots$ & -2.09 & 1.03 \\
\hline 339 & 3 & $\cdots$ & $15.38 \pm 0.10$ & $14.56 \pm 0.11$ & $14.21 \pm 0.24$ & $14.02 \pm 0.23$ & $\cdots$ & $\cdots$ & -2.14 & $\ldots$ \\
\hline 340 & 3 & $15.90 \pm 0.08$ & $14.57 \pm 0.05$ & $13.95 \pm 0.05$ & $13.06 \pm 0.11$ & $12.67 \pm 0.10$ & $12.52 \pm 0.52$ & $\cdots$ & -2.05 & 0.71 \\
\hline 341 & 3 & $14.83 \pm 0.17$ & & $12.32 \pm 0.10$ & $\ldots$ & $10.87 \pm 0.26$ & $\cdots$ & $\ldots$ & $\cdots$ & $\ldots$ \\
\hline 342 & 4 & $17.13 \pm 0.19$ & $15.55 \pm 0.12$ & $15.24 \pm 0.19$ & $\cdots$ & $13.45 \pm 0.13$ & $\cdots$ & $12.32 \pm 0.18$ & -1.04 & $\cdots$ \\
\hline 343 & 3 & $15.20 \pm 0.05$ & $13.70 \pm 0.03$ & $12.84 \pm 0.03$ & $11.82 \pm 0.06$ & $11.40 \pm 0.05$ & $11.44 \pm 0.42$ & $\ldots$ & -2.35 & 0.75 \\
\hline 347 & 3 & $15.90 \pm 0.08$ & $14.72 \pm 0.05$ & $13.90 \pm 0.05$ & $\cdots$ & $12.68 \pm 0.10$ & $\cdots$ & $\cdots$ & $\cdots$ & 0.28 \\
\hline 348 & 1 & $15.60 \pm 0.07$ & $14.90 \pm 0.06$ & $14.71 \pm 0.11$ & $\ldots$ & $13.04 \pm 0.19$ & $\cdots$ & $\cdots$ & $\cdots$ & $\ldots$ \\
\hline 349 & 1 & $16.86 \pm 0.16$ & $15.61 \pm 0.11$ & $14.73 \pm 0.11$ & $13.74 \pm 0.13$ & $13.36 \pm 0.13$ & $12.77 \pm 0.15$ & $11.99 \pm 0.39$ & -0.82 & $\cdots$ \\
\hline 350 & 3 & & $15.56 \pm 0.11$ & $14.47 \pm 0.09$ & $12.94 \pm 0.11$ & $12.45 \pm 0.10$ & $\ldots$ & $\ldots$ & -0.95 & $\ldots$ \\
\hline 355 & 3 & $13.21 \pm 0.03$ & $12.59 \pm 0.03$ & $12.31 \pm 0.03$ & $\cdots$ & $12.10 \pm 0.07$ & $\cdots$ & $11.50 \pm 0.19$ & -1.92 & $\cdots$ \\
\hline 357 & 4 & $=15.77 \pm 0.08$ & $14.22 \pm 0.04$ & $13.64 \pm 0.04$ & $\ldots$ & $13.20 \pm 0.12$ & $\ldots$ & $12.54 \pm 0.37$ & -1.87 & 1.00 \\
\hline 359 & 4 & $16.08 \pm 0.09$ & $14.77 \pm 0.07$ & $14.01 \pm 0.06$ & 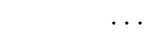 & $12.67 \pm 0.09$ & 1. & $11.83 \pm 0.19$ & -1.54 & 0.55 \\
\hline 360 & 3 & & $15.78 \pm 0.12$ & $13.65 \pm 0.05$ & $11.79 \pm 0.05$ & $11.12 \pm 0.05$ & $10.65 \pm 0.06$ & $10.20 \pm 0.13$ & -1.07 & $\cdots$ \\
\hline 361 & 3 & $9.52 \pm 0.02$ & $9.08 \pm 0.03$ & $8.92 \pm 0.02$ & $8.88 \pm 0.01$ & $8.89 \pm 0.02$ & $8.79 \pm 0.05$ & $8.51 \pm 0.16$ & -2.45 & $\ldots$ \\
\hline 363 & 3 & $16.80 \pm 0.18$ & $15.10 \pm 0.08$ & $13.98 \pm 0.09$ & $11.95 \pm 0.12$ & $11.70 \pm 0.13$ & $\cdots$ & $\cdots$ & -1.88 & $\cdots$ \\
\hline 364 & 4 & $16.45 \pm 0.16$ & $15.02 \pm 0.14$ & $14.66 \pm 0.11$ & $\cdots$ & $12.70 \pm 0.10$ & $\ldots$ & $12.00 \pm 0.29$ & -1.75 & $\cdots$ \\
\hline 365 & 3 & $13.63 \pm 0.03$ & $12.09 \pm 0.03$ & $10.96 \pm 0.02$ & $9.46 \pm 0.02$ & $8.94 \pm 0.02$ & $8.43 \pm 0.02$ & $7.50 \pm 0.04$ & -0.72 & 0.60 \\
\hline 367 & 3 & $13.46 \pm 0.02$ & $12.72 \pm 0.03$ & $12.42 \pm 0.02$ & $12.27 \pm 0.07$ & $12.24 \pm 0.08$ & $\ldots$ & $\ldots$ & -2.76 & 0.07 \\
\hline 368 & 3 & $\cdots$ & $15.69 \pm 0.13$ & $14.67 \pm 0.11$ & $13.20 \pm 0.13$ & $12.80 \pm 0.12$ & $\cdots$ & $\cdots$ & -1.31 & $\cdots$ \\
\hline 369 & 3 & $15.82 \pm 0.14$ & $14.79 \pm 0.09$ & $14.14 \pm 0.08$ & $13.25 \pm 0.11$ & $13.07 \pm 0.13$ & $\cdots$ & $\cdots$ & -2.14 & $\cdots$ \\
\hline 370 & 3 & $\cdots$ & $15.75 \pm 0.14$ & $13.98 \pm 0.06$ & $12.20 \pm 0.08$ & $12.07 \pm 0.10$ & $\cdots$ & $\cdots$ & -2.35 & $\cdots$ \\
\hline 371 & 2 & $16.95 \pm 0.19$ & $15.20 \pm 0.07$ & $14.39 \pm 0.08$ & $13.73 \pm 0.13$ & $13.30 \pm 0.12$ & $12.72 \pm 0.14$ & $11.85 \pm 0.19$ & -0.69 & $\ldots$ \\
\hline 374 & 3 & $16.78 \pm 0.20$ & $15.89 \pm 0.14$ & $14.79 \pm 0.13$ & $13.97 \pm 0.18$ & $13.70 \pm 0.21$ & $\ldots$ & $\ldots$ & -1.81 & $\ldots$ \\
\hline 376 & 3 & & $15.79 \pm 0.15$ & $14.24 \pm 0.07$ & $12.14 \pm 0.08$ & $11.58 \pm 0.06$ & $\ldots$ & $\cdots$ & -0.65 & $\ldots$ \\
\hline 377 & 1 & $15.99 \pm 0.12$ & $14.84 \pm 0.10$ & $14.23 \pm 0.09$ & $13.60 \pm 0.14$ & $13.24 \pm 0.13$ & $\cdots$ & $\cdots$ & -1.45 & . \\
\hline 378 & 3 & $16.31 \pm 0.11$ & $14.43 \pm 0.05$ & $13.74 \pm 0.05$ & $13.12 \pm 0.14$ & $13.07 \pm 0.15$ & $\ldots$ & $\cdots$ & -2.70 & $\ldots$ \\
\hline 379 & 3 & $\cdots$ & $14.94 \pm 0.07$ & $13.34 \pm 0.04$ & $12.13 \pm 0.07$ & $11.47 \pm 0.06$ & $11.21 \pm 0.30$ & $\cdots$ & -1.51 & 1.53 \\
\hline 383 & 3 & $\cdots$ & $15.66 \pm 0.13$ & $14.80 \pm 0.11$ & $13.51 \pm 0.12$ & $13.02 \pm 0.12$ & $12.81 \pm 0.59$ & $\cdots$ & -1.54 & $\cdots$ \\
\hline 384 & 3 & $15.65 \pm 0.12$ & $14.03 \pm 0.06$ & $13.05 \pm 0.06$ & $12.44 \pm 0.18$ & $12.02 \pm 0.19$ & $\ldots$ & $\ldots$ & -1.22 & $\ldots$ \\
\hline 385 & 3 & $\cdots$ & & $14.85 \pm 0.14$ & $\cdots$ & $12.53 \pm 0.35$ & $\cdots$ & $\cdots$ & $\cdots$ & $\cdots$ \\
\hline 386 & 4 & $17.01 \pm 0.19$ & $15.97 \pm 0.14$ & $15.16 \pm 0.14$ & $\cdots$ & $13.05 \pm 0.11$ & $\cdots$ & $12.03 \pm 0.34$ & -1.22 & 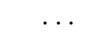 \\
\hline
\end{tabular}


Table 9-Continued

\begin{tabular}{|c|c|c|c|c|c|c|c|c|c|c|}
\hline $\begin{array}{l}S p . \\
\text { ID }\end{array}$ & $\begin{array}{l}\text { Ep. } \\
\text { ID }\end{array}$ & $\begin{array}{c}\mathrm{J} \\
{[\mathrm{mag} .]}\end{array}$ & $\begin{array}{c}\mathrm{H} \\
{[\mathrm{mag} .]}\end{array}$ & $\begin{array}{c}\mathrm{K} \\
{[\mathrm{mag} .]}\end{array}$ & $\begin{array}{l}3.6 \mu \mathrm{m} \\
\text { [mag.] }\end{array}$ & $\begin{array}{l}4.5 \mu \mathrm{m} \\
{[\mathrm{mag} .]}\end{array}$ & $\begin{array}{l}5.8 \mu \mathrm{m} \\
\text { [mag.] }\end{array}$ & $\begin{array}{l}8.0 \mu \mathrm{m} \\
{[\mathrm{mag} .]}\end{array}$ & $\alpha_{I R A C}$ & $\begin{array}{c}A_{K} \\
{[\mathrm{mag} .]}\end{array}$ \\
\hline 387 & 1 & $14.69 \pm 0.03$ & $13.42 \pm 0.02$ & $12.73 \pm 0.02$ & $11.92 \pm 0.08$ & $11.48 \pm 0.07$ & $10.15 \pm 0.13$ & $\ldots$ & 0.40 & 0.54 \\
\hline 389 & 1 & $13.34 \pm 0.04$ & $11.83 \pm 0.05$ & $10.70 \pm 0.03$ & $9.81 \pm 0.03$ & $9.49 \pm 0.03$ & $\cdots$ & $\ldots$ & -1.84 & 0.53 \\
\hline 390 & 3 & $15.67 \pm 0.09$ & $13.55 \pm 0.04$ & $12.19 \pm 0.03$ & $10.47 \pm 0.03$ & $9.83 \pm 0.03$ & $9.13 \pm 0.05$ & $8.11 \pm 0.12$ & -0.35 & 1.29 \\
\hline 391 & 3 & $\ldots$ & $15.90 \pm 0.16$ & $15.63 \pm 0.24$ & $14.44 \pm 0.21$ & $14.11 \pm 0.21$ & $\ldots$ & $\ldots$ & -1.54 & $\ldots$ \\
\hline 392 & 3 & $16.73 \pm 0.19$ & $14.92 \pm 0.08$ & $13.93 \pm 0.07$ & $12.62 \pm 0.16$ & $12.31 \pm 0.16$ & $\cdots$ & $\ldots$ & -1.64 & $\ldots$ \\
\hline 393 & 4 & & $15.95 \pm 0.15$ & $15.10 \pm 0.15$ & & $13.24 \pm 0.12$ & $\ldots$ & $12.79 \pm 0.32$ & -2.15 & $\ldots$ \\
\hline 394 & 3 & $13.53 \pm 0.10$ & $12.11 \pm 0.06$ & $11.23 \pm 0.04$ & $9.53 \pm 0.07$ & $9.03 \pm 0.12$ & $\ldots$ & $\ldots$ & -1.14 & 0.61 \\
\hline 395 & 1 & $14.71 \pm 0.05$ & $13.51 \pm 0.05$ & $13.02 \pm 0.04$ & $12.35 \pm 0.07$ & $12.12 \pm 0.07$ & $11.76 \pm 0.10$ & $10.87 \pm 0.13$ & -1.28 & 0.61 \\
\hline 397 & 3 & & $15.65 \pm 0.14$ & $14.72 \pm 0.11$ & $13.75 \pm 0.15$ & $13.59 \pm 0.16$ & $\cdots$ & $\ldots$ & -2.24 & $\ldots$ \\
\hline 399 & 3 & $14.64 \pm 0.04$ & $13.28 \pm 0.04$ & $12.56 \pm 0.05$ & $11.87 \pm 0.09$ & $11.52 \pm 0.09$ & $\cdots$ & $\cdots$ & -1.75 & 0.67 \\
\hline 401 & 1 & $16.89 \pm 0.18$ & $15.22 \pm 0.06$ & $14.36 \pm 0.06$ & $13.65 \pm 0.12$ & $13.19 \pm 0.12$ & $12.89 \pm 0.15$ & $12.01 \pm 0.19$ & -1.04 & $\ldots$ \\
\hline 402 & 3 & $16.34 \pm 0.16$ & $13.94 \pm 0.05$ & $12.89 \pm 0.04$ & $13.35 \pm 1.35$ & $12.33 \pm 0.70$ & $\ldots$ & $\ldots$ & 1.12 & $\ldots$ \\
\hline 405 & 1 & $15.90 \pm 0.08$ & $14.37 \pm 0.06$ & $13.54 \pm 0.05$ & $12.50 \pm 0.07$ & $12.13 \pm 0.07$ & $11.79 \pm 0.08$ & $11.05 \pm 0.09$ & -1.36 & 0.83 \\
\hline 406 & 4 & $16.42 \pm 0.13$ & $15.11 \pm 0.08$ & $14.76 \pm 0.13$ & $13.82 \pm 0.17$ & $13.49 \pm 0.17$ & $\ldots$ & $\ldots$ & -1.59 & $\ldots$ \\
\hline 407 & 3 & $16.97 \pm 0.22$ & $15.45 \pm 0.10$ & $14.98 \pm 0.15$ & $14.00 \pm 0.37$ & $13.76 \pm 0.33$ & $\ldots$ & $\ldots$ & -1.92 & $\ldots$ \\
\hline 408 & 3 & $\cdots$ & & $14.29 \pm 0.10$ & & $13.00 \pm 0.15$ & $\cdots$ & $\cdots$ & & $\ldots$ \\
\hline 409 & 3 & $16.97 \pm 0.19$ & $15.86 \pm 0.16$ & $15.19 \pm 0.18$ & $13.65 \pm 0.19$ & $13.50 \pm 0.17$ & $\cdots$ & $\cdots$ & -2.31 & $\cdots$ \\
\hline 410 & 4 & & $15.38 \pm 0.12$ & $14.95 \pm 0.14$ & & $13.42 \pm 0.14$ & $\cdots$ & $\cdots$ & & $\ldots$ \\
\hline 411 & 3 & $16.66 \pm 0.15$ & $14.95 \pm 0.07$ & $13.88 \pm 0.08$ & $12.47 \pm 0.07$ & $11.98 \pm 0.07$ & $11.71 \pm 0.11$ & $10.15 \pm 0.21$ & -0.40 & 0.74 \\
\hline 412 & 3 & $\cdots$ & $15.61 \pm 0.14$ & $13.53 \pm 0.05$ & $12.15 \pm 0.15$ & $11.62 \pm 0.11$ & $\cdots$ & $\cdots$ & -0.81 & $\cdots$ \\
\hline 413 & 3 & $\ldots$ & $15.53 \pm 0.12$ & $14.50 \pm 0.09$ & $13.81 \pm 0.14$ & $13.62 \pm 0.16$ & $\ldots$ & $\ldots$ & -2.11 & $\ldots$ \\
\hline 415 & 3 & $13.97 \pm 0.03$ & $12.81 \pm 0.03$ & $12.21 \pm 0.02$ & $11.84 \pm 0.05$ & $11.72 \pm 0.06$ & $11.50 \pm 0.17$ & $\cdots$ & -2.35 & 0.46 \\
\hline 416 & 3 & $14.60 \pm 0.04$ & $13.60 \pm 0.05$ & $13.12 \pm 0.04$ & $12.75 \pm 0.09$ & $12.58 \pm 0.10$ & $\ldots$ & $\ldots$ & -2.33 & 0.31 \\
\hline 417 & 3 & $16.78 \pm 0.17$ & $14.80 \pm 0.07$ & $13.56 \pm 0.05$ & $12.70 \pm 0.18$ & $12.63 \pm 0.23$ & $\ldots$ & $\cdots$ & -2.57 & $\cdots$ \\
\hline 418 & 3 & $12.99 \pm 0.04$ & $12.22 \pm 0.04$ & $11.68 \pm 0.04$ & $11.38 \pm 0.04$ & $11.18 \pm 0.05$ & $10.70 \pm 0.10$ & $9.87 \pm 0.19$ & -1.11 & $\ldots$ \\
\hline 419 & 2 & $16.22 \pm 0.11$ & $15.30 \pm 0.09$ & $14.87 \pm 0.13$ & & $13.30 \pm 0.14$ & $\cdots$ & $\cdots$ & . & $\cdots$ \\
\hline 420 & 1 & $15.31 \pm 0.06$ & $14.19 \pm 0.04$ & $13.36 \pm 0.04$ & $12.41 \pm 0.07$ & $12.12 \pm 0.07$ & $11.81 \pm 0.13$ & $11.09 \pm 0.29$ & -1.41 & 0.19 \\
\hline 421 & 1 & $16.26 \pm 0.12$ & $15.44 \pm 0.10$ & $14.84 \pm 0.13$ & $13.86 \pm 0.14$ & $13.54 \pm 0.14$ & $12.77 \pm 0.19$ & $\cdots$ & -0.77 & $\cdots$ \\
\hline 422 & 1 & $16.67 \pm 0.16$ & $15.88 \pm 0.13$ & $15.27 \pm 0.17$ & & $13.80 \pm 0.16$ & $\cdots$ & $\cdots$ & $\ldots$ & $\cdots$ \\
\hline 423 & 3 & $16.42 \pm 0.12$ & $15.23 \pm 0.09$ & $14.96 \pm 0.14$ & $14.71 \pm 0.21$ & $14.60 \pm 0.23$ & $\ldots$ & $\ldots$ & -2.44 & $\ldots$ \\
\hline 424 & 3 & $\cdots$ & $15.29 \pm 0.11$ & $13.99 \pm 0.07$ & $\cdots$ & $10.94 \pm 0.04$ & $\cdots$ & $10.34 \pm 0.26$ & -1.91 & $\cdots$ \\
\hline 425 & 1 & $\ldots$ & $\ldots$ & $15.07 \pm 0.14$ & $13.85 \pm 0.14$ & $13.23 \pm 0.12$ & $12.86 \pm 0.14$ & $12.23 \pm 0.15$ & -1.06 & $\ldots$ \\
\hline 426 & 1 & $15.06 \pm 0.05$ & $13.81 \pm 0.04$ & $13.33 \pm 0.04$ & $12.74 \pm 0.08$ & $12.45 \pm 0.08$ & $12.11 \pm 0.10$ & $11.43 \pm 0.14$ & -1.48 & 0.68 \\
\hline 428 & 1 & & $15.62 \pm 0.12$ & $14.79 \pm 0.12$ & $13.66 \pm 0.13$ & $13.29 \pm 0.12$ & $12.89 \pm 0.14$ & $12.32 \pm 0.16$ & -1.33 & $\ldots$ \\
\hline 429 & 1 & $16.11 \pm 0.10$ & $14.94 \pm 0.07$ & $14.22 \pm 0.08$ & $13.78 \pm 0.13$ & $13.43 \pm 0.13$ & $13.17 \pm 0.16$ & $12.58 \pm 0.18$ & -1.58 & 0.37 \\
\hline 430 & 3 & $14.74 \pm 0.04$ & $13.00 \pm 0.03$ & $12.24 \pm 0.03$ & $11.60 \pm 0.06$ & $11.50 \pm 0.06$ & $10.95 \pm 0.32$ & $\cdots$ & -1.93 & 1.20 \\
\hline 431 & 3 & $17.13 \pm 0.24$ & $15.55 \pm 0.11$ & $14.90 \pm 0.13$ & $\cdots$ & $12.97 \pm 0.11$ & $\cdots$ & $\cdots$ & $\ldots$ & $\cdots$ \\
\hline 432 & 3 & $16.36 \pm 0.11$ & $15.40 \pm 0.11$ & $14.89 \pm 0.13$ & $14.68 \pm 0.21$ & $14.38 \pm 0.22$ & $\cdots$ & $\ldots$ & -1.70 & $\ldots$ \\
\hline 433 & 3 & $15.05 \pm 0.05$ & $13.86 \pm 0.04$ & $13.14 \pm 0.04$ & $\ldots$ & $11.79 \pm 0.06$ & $\ldots$ & $10.24 \pm 0.13$ & -0.37 & 0.39 \\
\hline 435 & 3 & $16.66 \pm 0.18$ & $15.53 \pm 0.11$ & $14.98 \pm 0.15$ & $\cdots$ & $13.50 \pm 0.14$ & $\ldots$ & $\ldots$ & $\ldots$ & $\cdots$ \\
\hline 436 & 1 & $16.58 \pm 0.13$ & $15.79 \pm 0.14$ & $\cdots$ & $14.59 \pm 0.20$ & $14.09 \pm 0.19$ & $\cdots$ & $\cdots$ & -0.88 & $\cdots$ \\
\hline 441 & 3 & $11.12 \pm 0.02$ & $10.17 \pm 0.02$ & $9.18 \pm 0.02$ & $\cdots$ & $7.21 \pm 0.01$ & $\cdots$ & $6.22 \pm 0.02$ & -1.27 & $\cdots$ \\
\hline 442 & 1 & $15.52 \pm 0.06$ & $14.72 \pm 0.07$ & $14.11 \pm 0.06$ & $13.54 \pm 0.17$ & $13.09 \pm 0.18$ & $\ldots$ & $\ldots$ & -1.13 & $\ldots$ \\
\hline 444 & 3 & $\cdots$ & $15.58 \pm 0.13$ & $\cdots$ & $14.31 \pm 0.18$ & $14.21 \pm 0.20$ & $\cdots$ & $\cdots$ & -2.47 & . \\
\hline 445 & 3 & $16.07 \pm 0.10$ & $14.84 \pm 0.06$ & $14.24 \pm 0.08$ & 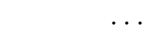 & $12.80 \pm 0.12$ & $\cdots$ & $\cdots$ & $\cdots$ & $\cdots$ \\
\hline
\end{tabular}


Table 9 - Continued

\begin{tabular}{|c|c|c|c|c|c|c|c|c|c|c|}
\hline $\begin{array}{l}S p . \\
\text { ID }\end{array}$ & $\begin{array}{c}\text { Ep. } \\
\text { ID }\end{array}$ & $\begin{array}{c}\mathrm{J} \\
{[\mathrm{mag} .]}\end{array}$ & $\begin{array}{c}\mathrm{H} \\
{[\mathrm{mag} .]}\end{array}$ & $\begin{array}{c}\mathrm{K} \\
{[\mathrm{mag} .]}\end{array}$ & $\begin{array}{l}3.6 \mu \mathrm{m} \\
\text { [mag.] }\end{array}$ & $\begin{array}{l}4.5 \mu \mathrm{m} \\
\text { [mag.] }\end{array}$ & $\begin{array}{l}5.8 \mu m \\
\text { [mag.] }\end{array}$ & $\begin{array}{l}8.0 \mu \mathrm{m} \\
\text { [mag.] }\end{array}$ & $\alpha_{I R A C}$ & $\begin{array}{c}A_{K} \\
\text { [mag.] }\end{array}$ \\
\hline 446 & 1 & $1 \quad 16.63 \pm 0.16$ & $15.10 \pm 0.10$ & $14.31 \pm 0.09$ & $13.30 \pm 0.10$ & $12.87 \pm 0.10$ & $12.44 \pm 0.11$ & $11.77 \pm 0.16$ & -1.12 & \\
\hline 448 & 1 & $1 \quad 15.30 \pm 0.07$ & $14.17 \pm 0.06$ & $13.40 \pm 0.05$ & $12.03 \pm 0.06$ & $11.52 \pm 0.05$ & $11.15 \pm 0.06$ & $10.39 \pm 0.07$ & -1.06 & 0.27 \\
\hline 449 & 1 & $1 \quad 15.17 \pm 0.06$ & $13.99 \pm 0.04$ & $13.31 \pm 0.04$ & $12.79 \pm 0.09$ & $12.52 \pm 0.09$ & $12.27 \pm 0.29$ & $\ldots$ & -2.00 & 0.43 \\
\hline 450 & 3 & $\cdots$ & $15.85 \pm 0.19$ & $15.19 \pm 0.19$ & $\cdots$ & $13.30 \pm 0.13$ & $\cdots$ & $12.78 \pm 0.22$ & -2.05 & $\cdots$ \\
\hline 451 & 4 & $4 \quad 14.35 \pm 0.03$ & $12.99 \pm 0.03$ & $12.18 \pm 0.03$ & $11.00 \pm 0.04$ & & $10.34 \pm 0.04$ & $\ldots$ & -1.76 & 0.58 \\
\hline 452 & 1 & 1 & $15.00 \pm 0.07$ & $13.29 \pm 0.05$ & $11.69 \pm 0.05$ & $10.86 \pm 0.04$ & $10.25 \pm 0.04$ & $9.51 \pm 0.04$ & -0.59 & 1.25 \\
\hline 456 & 3 & $\begin{array}{ll}3 & 13.38 \pm 0.02\end{array}$ & $12.63 \pm 0.03$ & $12.19 \pm 0.03$ & & $10.82 \pm 0.04$ & & $9.45 \pm 0.07$ & -0.64 & $\ldots$ \\
\hline 457 & 1 & $1 \quad 15.98 \pm 0.09$ & $13.90 \pm 0.04$ & $12.39 \pm 0.03$ & $10.55 \pm 0.03$ & $9.92 \pm 0.03$ & $9.32 \pm 0.03$ & $8.34 \pm 0.02$ & -0.50 & 1.08 \\
\hline 461 & 1 & $1 \quad 16.53 \pm 0.14$ & $14.93 \pm 0.07$ & $13.93 \pm 0.06$ & $\ldots$ & $12.41 \pm 0.08$ & $12.17 \pm 0.10$ & $11.51 \pm 0.14$ & -1.43 & 0.75 \\
\hline 462 & 3 & $\begin{array}{ll}3 & 12.01 \pm 0.02\end{array}$ & $11.31 \pm 0.03$ & $10.84 \pm 0.02$ & $\cdots$ & $9.95 \pm 0.03$ & $\cdots$ & $9.40 \pm 0.04$ & -1.99 & $\cdots$ \\
\hline 464 & 2 & $2 \quad 15.80 \pm 0.11$ & $14.60 \pm 0.07$ & $14.02 \pm 0.07$ & $12.91 \pm 0.10$ & $12.68 \pm 0.11$ & $11.82 \pm 0.32$ & $\ldots$ & -0.79 & $\cdots$ \\
\hline 465 & 3 & $\begin{array}{ll}3 & 15.66 \pm 0.07\end{array}$ & $14.38 \pm 0.05$ & $14.19 \pm 0.08$ & $13.96 \pm 0.15$ & $13.91 \pm 0.17$ & $\ldots$ & $\ldots$ & -2.95 & 0.72 \\
\hline 469 & 1 & $\cdots$ & $\ldots$ & $\cdots$ & $13.90 \pm 0.14$ & $13.37 \pm 0.13$ & $12.94 \pm 0.15$ & $12.30 \pm 0.17$ & -1.05 & $\cdots$ \\
\hline 470 & 2 & $\cdots$ & $\cdots$ & $\cdots$ & $12.69 \pm 0.08$ & $12.29 \pm 0.08$ & $11.93 \pm 0.10$ & $10.96 \pm 0.10$ & -0.91 & $\cdots$ \\
\hline 473 & 3 & $\cdots$ & $\cdots$ & $\cdots$ & $14.81 \pm 0.31$ & $14.47 \pm 0.24$ & $\ldots$ & $\ldots$ & -1.53 & $\ldots$ \\
\hline 474 & 2 & $\ldots$ & $\ldots$ & $\ldots$ & $13.12 \pm 0.10$ & $12.22 \pm 0.08$ & $11.69 \pm 0.10$ & $11.03 \pm 0.24$ & -0.52 & $\ldots$ \\
\hline 476 & 3 & $\ldots$ & $\ldots$ & $\ldots$ & $11.52 \pm 0.22$ & $10.88 \pm 0.15$ & $\cdots$ & $\cdots$ & -0.38 & $\ldots$ \\
\hline 479 & 3 & $\cdots$ & $\ldots$ & $\cdots$ & $14.00 \pm 0.22$ & $13.91 \pm 0.28$ & $\ldots$ & $\ldots$ & -2.49 & $\cdots$ \\
\hline 480 & 2 & $\ldots$ & $\ldots$ & $\ldots$ & $13.38 \pm 0.11$ & $12.61 \pm 0.09$ & $12.12 \pm 0.11$ & $11.45 \pm 0.13$ & -0.69 & $\ldots$ \\
\hline 483 & 3 & $\cdots$ & $\ldots$ & $\cdots$ & $13.81 \pm 0.26$ & $13.78 \pm 0.30$ & $\ldots$ & $\ldots$ & -2.74 & $\cdots$ \\
\hline 485 & 3 & $\ldots$ & $\ldots$ & $\ldots$ & $13.96 \pm 0.14$ & $13.41 \pm 0.14$ & $12.84 \pm 0.16$ & $12.05 \pm 0.23$ & -0.67 & $\ldots$ \\
\hline 486 & 1 & $\cdots$ & $\ldots$ & $\cdots$ & $11.50 \pm 0.05$ & $11.10 \pm 0.05$ & $10.72 \pm 0.07$ & $=0.12$ & -1.22 & $\cdots$ \\
\hline 487 & 1 & $\ldots$ & $\ldots$ & $\ldots$ & $11.82 \pm 0.05$ & $11.25 \pm 0.05$ & $10.93 \pm 0.06$ & $10.12 \pm 0.09$ & -0.98 & $\ldots$ \\
\hline 488 & 3 & $\cdots$ & $\ldots$ & $\cdots$ & $12.22 \pm 0.06$ & $11.97 \pm 0.07$ & $11.53 \pm 0.10$ & $10.88 \pm 0.19$ & -1.31 & $\cdots$ \\
\hline 489 & 3 & $\ldots$ & $\ldots$ & $\ldots$ & $14.06 \pm 0.17$ & $13.85 \pm 0.28$ & $\ldots$ & $\ldots$ & -2.04 & $\ldots$ \\
\hline 491 & 3 & $\cdots$ & $\ldots$ & $\cdots$ & $12.53 \pm 0.12$ & $11.97 \pm 0.10$ & $\cdots$ & $\cdots$ & -0.64 & $\ldots$ \\
\hline 494 & 4 & $\ldots$ & $\ldots$ & $\ldots$ & $11.49 \pm 0.26$ & $10.89 \pm 0.24$ & $\ldots$ & $\ldots$ & -0.53 & $\ldots$ \\
\hline 495 & 2 & $2 \quad 16.72 \pm 0.19$ & $15.59 \pm 0.13$ & $15.27 \pm 0.18$ & $14.22 \pm 0.16$ & $13.89 \pm 0.16$ & $13.56 \pm 0.24$ & $12.68 \pm 0.44$ & -1.13 & $\cdots$ \\
\hline 496 & 2 & $214.85 \pm 0.04$ & $13.67 \pm 0.03$ & $13.12 \pm 0.03$ & $12.59 \pm 0.08$ & $12.14 \pm 0.07$ & $11.90 \pm 0.09$ & $11.26 \pm 0.14$ & -1.49 & 0.55 \\
\hline 497 & 2 & $\ldots$ & $15.56 \pm 0.12$ & $14.62 \pm 0.09$ & $13.79 \pm 0.13$ & $13.34 \pm 0.13$ & $13.07 \pm 0.16$ & $12.41 \pm 0.17$ & -1.34 & $\ldots$ \\
\hline 498 & 2 & $2 \quad 15.63 \pm 0.07$ & $14.19 \pm 0.05$ & $13.33 \pm 0.04$ & $12.26 \pm 0.07$ & $11.85 \pm 0.06$ & $11.59 \pm 0.08$ & $10.80 \pm 0.08$ & -1.34 & 0.68 \\
\hline 499 & 2 & $2 \quad 15.60 \pm 0.07$ & $14.57 \pm 0.06$ & $14.06 \pm 0.07$ & $13.12 \pm 0.10$ & $12.73 \pm 0.10$ & $12.40 \pm 0.11$ & $11.54 \pm 0.11$ & -1.14 & 0.35 \\
\hline 500 & 3 & $\begin{array}{ll}3 & 15.35 \pm 0.05\end{array}$ & $13.88 \pm 0.04$ & $13.09 \pm 0.04$ & $12.42 \pm 0.12$ & $\cdots$ & $\cdots$ & $\cdots$ & $\cdots$ & 0.77 \\
\hline 501 & 3 & $\begin{array}{ll}3 & 15.83 \pm 0.09\end{array}$ & $14.19 \pm 0.06$ & $13.37 \pm 0.06$ & $12.07 \pm 0.08$ & $11.89 \pm 0.09$ & $\cdots$ & $\cdots$ & -2.56 & 1.02 \\
\hline 503 & 2 & $214.53 \pm 0.03$ & $13.43 \pm 0.03$ & $12.86 \pm 0.03$ & $\cdots$ & $11.24 \pm 0.05$ & $\cdots$ & $10.29 \pm 0.06$ & -1.36 & 0.40 \\
\hline 504 & 2 & $2 \quad 15.52 \pm 0.05$ & $14.20 \pm 0.04$ & $13.63 \pm 0.04$ & $13.02 \pm 0.09$ & $12.72 \pm 0.10$ & $12.58 \pm 0.13$ & $11.90 \pm 0.14$ & -1.75 & 0.75 \\
\hline 506 & 2 & $2 \quad 16.87 \pm 0.18$ & $15.21 \pm 0.13$ & $14.41 \pm 0.09$ & $13.92 \pm 0.14$ & $13.42 \pm 0.13$ & $13.14 \pm 0.15$ & $12.31 \pm 0.17$ & -1.07 & $\cdots$ \\
\hline 507 & 2 & $2 \quad 17.33 \pm 0.26$ & $16.21 \pm 0.18$ & $15.43 \pm 0.20$ & $\ldots$ & $13.31 \pm 0.13$ & $\ldots$ & $12.31 \pm 0.16$ & -1.25 & $\cdots$ \\
\hline 508 & 2 & $2 \quad 15.13 \pm 0.06$ & $13.84 \pm 0.06$ & $13.04 \pm 0.06$ & $\cdots$ & $11.78 \pm 0.06$ & $\cdots$ & $11.06 \pm 0.10$ & -1.75 & 0.49 \\
\hline 509 & 2 & $2 \quad 16.58 \pm 0.12$ & $15.14 \pm 0.08$ & $14.48 \pm 0.09$ & $13.27 \pm 0.10$ & $12.84 \pm 0.10$ & $12.39 \pm 0.11$ & $11.54 \pm 0.12$ & -0.89 & $\ldots$ \\
\hline 510 & 2 & $\cdots$ & $15.19 \pm 0.16$ & $14.56 \pm 0.12$ & $13.13 \pm 0.10$ & $12.60 \pm 0.09$ & $12.34 \pm 0.12$ & $12.18 \pm 0.23$ & -1.82 & $\cdots$ \\
\hline 512 & 3 & $\cdots$ & $15.83 \pm 0.14$ & $14.50 \pm 0.10$ & $13.53 \pm 0.20$ & $13.19 \pm 0.20$ & $\cdots$ & $\cdots$ & -1.55 & $\cdots$ \\
\hline 513 & 2 & $\begin{array}{ll}2 & 16.27 \pm 0.10\end{array}$ & $14.98 \pm 0.05$ & $14.39 \pm 0.08$ & $13.84 \pm 0.13$ & $13.47 \pm 0.13$ & $12.94 \pm 0.15$ & $11.95 \pm 0.16$ & -0.79 & 0.68 \\
\hline 514 & 3 & $\begin{array}{ll}3 & 13.96 \pm 0.03\end{array}$ & $12.83 \pm 0.03$ & $12.38 \pm 0.03$ & $12.02 \pm 0.06$ & $11.97 \pm 0.07$ & $11.34 \pm 0.21$ & $\cdots$ & -1.70 & 0.55 \\
\hline 515 & 2 & $214.18 \pm 0.03$ & $13.00 \pm 0.02$ & $12.15 \pm 0.03$ & $11.40 \pm 0.04$ & $10.98 \pm 0.04$ & $10.55 \pm 0.05$ & $9.55 \pm 0.04$ & -0.80 & 0.26 \\
\hline
\end{tabular}


Table 9-Continued

\begin{tabular}{|c|c|c|c|c|c|c|c|c|c|c|}
\hline $\begin{array}{l}S p . \\
\text { ID }\end{array}$ & $\begin{array}{l}\text { Ep. } \\
\text { ID }\end{array}$ & $\begin{array}{c}\mathrm{J} \\
{[\mathrm{mag} .]}\end{array}$ & $\begin{array}{c}\mathrm{H} \\
{[\mathrm{mag} .]}\end{array}$ & $\begin{array}{c}\mathrm{K} \\
{[\mathrm{mag} .]}\end{array}$ & $\begin{array}{l}3.6 \mu \mathrm{m} \\
\text { [mag.] }\end{array}$ & $\begin{array}{l}4.5 \mu \mathrm{m} \\
{[\mathrm{mag} .]}\end{array}$ & $\begin{array}{l}5.8 \mu \mathrm{m} \\
\text { [mag.] }\end{array}$ & $\begin{array}{l}8.0 \mu \mathrm{m} \\
{[\mathrm{mag} .]}\end{array}$ & $\alpha_{I R A C}$ & $\begin{array}{c}A_{K} \\
{[\mathrm{mag} .]}\end{array}$ \\
\hline 518 & 3 & $16.62 \pm 0.17$ & $14.39 \pm 0.05$ & $13.30 \pm 0.04$ & $12.52 \pm 0.08$ & $12.22 \pm 0.08$ & $11.98 \pm 0.19$ & & -2.24 & 1.45 \\
\hline 519 & 2 & $14.74 \pm 0.05$ & $13.49 \pm 0.04$ & $12.85 \pm 0.03$ & $\ldots$ & $11.31 \pm 0.05$ & $\ldots$ & $10.53 \pm 0.07$ & -1.64 & 0.58 \\
\hline 520 & 2 & $15.60 \pm 0.07$ & $14.66 \pm 0.08$ & $14.02 \pm 0.08$ & $\ldots$ & $12.64 \pm 0.09$ & $\ldots$ & $11.54 \pm 0.12$ & -1.08 & 0.09 \\
\hline 521 & 2 & $15.60 \pm 0.06$ & $14.31 \pm 0.06$ & $13.57 \pm 0.05$ & $\cdots$ & $12.02 \pm 0.07$ & $\cdots$ & $11.62 \pm 0.12$ & -2.28 & 0.53 \\
\hline 522 & 2 & $16.54 \pm 0.13$ & $15.02 \pm 0.06$ & $14.48 \pm 0.08$ & $\ldots$ & $12.91 \pm 0.10$ & $\ldots$ & $12.14 \pm 0.14$ & -1.63 & $\ldots$ \\
\hline 523 & 3 & & $15.34 \pm 0.11$ & $13.54 \pm 0.07$ & $12.44 \pm 0.07$ & $12.14 \pm 0.07$ & $12.16 \pm 0.16$ & & -2.34 & $\cdots$ \\
\hline 525 & 2 & $15.73 \pm 0.07$ & $14.36 \pm 0.05$ & $13.70 \pm 0.05$ & $12.99 \pm 0.09$ & $12.60 \pm 0.09$ & $12.16 \pm 0.10$ & $11.19 \pm 0.09$ & -0.93 & 0.75 \\
\hline 526 & 2 & $14.69 \pm 0.05$ & $13.52 \pm 0.05$ & $12.77 \pm 0.04$ & & $11.57 \pm 0.06$ & $\ldots$ & $10.64 \pm 0.07$ & -1.39 & 0.35 \\
\hline 527 & 2 & $16.75 \pm 0.16$ & $15.46 \pm 0.09$ & $14.64 \pm 0.11$ & $13.80 \pm 0.13$ & $13.38 \pm 0.13$ & $13.08 \pm 0.17$ & $12.21 \pm 0.23$ & -1.09 & $\ldots$ \\
\hline 528 & 2 & $\ldots$ & $15.72 \pm 0.15$ & $14.69 \pm 0.10$ & $13.75 \pm 0.13$ & $13.36 \pm 0.13$ & $13.03 \pm 0.15$ & $12.45 \pm 0.17$ & -1.40 & $\cdots$ \\
\hline 529 & 2 & $\cdots$ & $15.90 \pm 0.13$ & $14.38 \pm 0.08$ & $12.31 \pm 0.07$ & $11.83 \pm 0.06$ & $11.38 \pm 0.07$ & $10.72 \pm 0.08$ & -1.06 & $\cdots$ \\
\hline 530 & 3 & $15.07 \pm 0.04$ & $14.50 \pm 0.02$ & $14.23 \pm 0.06$ & $13.54 \pm 0.13$ & $13.21 \pm 0.13$ & $\ldots$ & $\ldots$ & & $\ldots$ \\
\hline 532 & 2 & $15.84 \pm 0.07$ & $14.69 \pm 0.05$ & $14.30 \pm 0.08$ & $13.73 \pm 0.13$ & $13.40 \pm 0.13$ & $12.78 \pm 0.13$ & $11.71 \pm 0.12$ & -0.60 & 0.57 \\
\hline 533 & 2 & $16.52 \pm 0.12$ & $15.17 \pm 0.11$ & $14.49 \pm 0.09$ & $\ldots$ & $13.01 \pm 0.11$ & $\ldots$ & $12.32 \pm 0.17$ & -1.76 & $\ldots$ \\
\hline 534 & 2 & $15.37 \pm 0.05$ & $13.86 \pm 0.02$ & $13.17 \pm 0.03$ & $\ldots$ & $11.87 \pm 0.06$ & $\ldots$ & $11.15 \pm 0.10$ & -1.76 & 0.94 \\
\hline 535 & 2 & $15.50 \pm 0.07$ & $14.29 \pm 0.04$ & $13.54 \pm 0.05$ & $12.84 \pm 0.08$ & $12.52 \pm 0.09$ & $12.33 \pm 0.11$ & $11.73 \pm 0.12$ & -1.71 & 0.40 \\
\hline 537 & 2 & $16.18 \pm 0.10$ & $15.01 \pm 0.09$ & $14.62 \pm 0.11$ & & $12.99 \pm 0.11$ & $\ldots$ & $11.79 \pm 0.13$ & -0.92 & . \\
\hline 538 & 2 & & $15.23 \pm 0.09$ & $15.07 \pm 0.16$ & $13.90 \pm 0.14$ & $13.73 \pm 0.15$ & $\cdots$ & $\ldots$ & -2.17 & $\ldots$ \\
\hline 539 & 2 & $14.88 \pm 0.06$ & $13.51 \pm 0.05$ & $12.79 \pm 0.03$ & $11.49 \pm 0.05$ & $11.08 \pm 0.04$ & $10.80 \pm 0.05$ & $10.02 \pm 0.06$ & -1.33 & 0.68 \\
\hline 540 & 3 & $\cdots$ & $13.57 \pm 0.05$ & $\cdots$ & $11.94 \pm 0.09$ & $11.72 \pm 0.08$ & $\cdots$ & $\cdots$ & -2.00 & $\cdots$ \\
\hline 541 & 2 & $16.92 \pm 0.29$ & $15.24 \pm 0.11$ & $14.43 \pm 0.11$ & $13.58 \pm 0.12$ & $13.20 \pm 0.12$ & $13.00 \pm 0.14$ & $12.45 \pm 0.16$ & -1.62 & $\ldots$ \\
\hline 542 & 2 & $17.05 \pm 0.21$ & $15.16 \pm 0.06$ & $14.40 \pm 0.07$ & $13.36 \pm 0.11$ & 12.8 & 0.12 & \pm 0.15 & -1.52 & $\cdots$ \\
\hline 544 & 2 & $16.53 \pm 0.13$ & $15.21 \pm 0.08$ & $14.32 \pm 0.08$ & $13.42 \pm 0.11$ & $13.08 \pm 0.11$ & $12.73 \pm 0.15$ & $11.74 \pm 0.21$ & -0.97 & $\ldots$ \\
\hline 545 & 2 & $12.34 \pm 0.04$ & $11.42 \pm 0.04$ & $10.85 \pm 0.03$ & $10.33 \pm 0.03$ & $9.81 \pm 0.03$ & $9.48 \pm 0.03$ & $8.81 \pm 0.03$ & -1 & 0.11 \\
\hline 546 & 2 & & $15.63 \pm 0.11$ & $15.50 \pm 0.23$ & $14.32 \pm 0.17$ & $14.00 \pm 0.17$ & $13.64 \pm 0.33$ & $\ldots$ & -1.58 & $\ldots$ \\
\hline 547 & 2 & 16.8 & $15.27 \pm 0.10$ & $14.61 \pm 0.10$ & $13.33 \pm 0.11$ & $12.98 \pm 0.11$ & $12.77 \pm 0.16$ & $0 \pm 0.30$ & -1.51 & $\cdots$ \\
\hline 548 & 2 & $15.23 \pm 0.06$ & $13.96 \pm 0.04$ & $13.43 \pm 0.04$ & $12.58 \pm 0.08$ & $12.09 \pm 0.07$ & $11.75 \pm 0.09$ & $11.29 \pm 0.19$ & -1.53 & 0.71 \\
\hline 549 & 2 & $\cdots$ & $15.23 \pm 0.08$ & $\cdots$ & $13.40 \pm 0.11$ & $12.85 \pm 0.10$ & $12.49 \pm 0.12$ & $11.85 \pm 0.17$ & -1.13 & $\cdots$ \\
\hline 550 & 2 & $\cdots$ & $15.26 \pm 0.10$ & $14.15 \pm 0.10$ & $\ldots$ & $12.15 \pm 0.18$ & $\cdots$ & $\cdots$ & $\ldots$ & $\cdots$ \\
\hline 551 & 3 & $\ldots$ & $15.77 \pm 0.14$ & $14.96 \pm 0.15$ & $\ldots$ & $13.13 \pm 0.12$ & $\ldots$ & $\ldots$ & $\ldots$ & $\ldots$ \\
\hline 552 & 3 & $15.47 \pm 0.05$ & $14.30 \pm 0.05$ & $13.51 \pm 0.04$ & $12.61 \pm 0.08$ & $12.42 \pm 0.08$ & $12.34 \pm 0.11$ & $\cdots$ & -2.45 & 0.31 \\
\hline 556 & 4 & $\ldots$ & $\ldots$ & $14.90 \pm 0.11$ & $13.29 \pm 0.11$ & $12.56 \pm 0.09$ & $12.40 \pm 0.14$ & $11.85 \pm 0.24$ & -1.33 & $\ldots$ \\
\hline 557 & 2 & $16.79 \pm 0.16$ & $15.52 \pm 0.11$ & $15.04 \pm 0.13$ & $\cdots$ & $13.57 \pm 0.15$ & $\cdots$ & $\cdots$ & $\cdots$ & $\cdots$ \\
\hline 558 & 4 & $16.33 \pm 0.13$ & $15.67 \pm 0.12$ & $14.86 \pm 0.14$ & $=0.19$ & $14.10 \pm 0.21$ & $\ldots$ & $\ldots$ & -2.12 & $\ldots$ \\
\hline 560 & 3 & $15.74 \pm 0.10$ & $14.24 \pm 0.04$ & $13.31 \pm 0.04$ & $11.99 \pm 0.22$ & $11.62 \pm 0.22$ & $\cdots$ & $\cdots$ & -1.39 & $\cdots$ \\
\hline 563 & 3 & $\ldots$ & $15.80 \pm 0.13$ & $15.10 \pm 0.13$ & $13.80 \pm 0.14$ & $13.35 \pm 0.13$ & $\ldots$ & $\cdots$ & -1.11 & $\ldots$ \\
\hline 564 & 3 & $\cdots$ & $15.67 \pm 0.13$ & $14.69 \pm 0.12$ & $13.34 \pm 0.12$ & $12.99 \pm 0.15$ & $\cdots$ & $\cdots$ & -1.50 & $\cdots$ \\
\hline 565 & 4 & $=14.00 \pm 0.10$ & $12.31 \pm 0.11$ & $10.61 \pm 0.09$ & $\cdots$ & $8.52 \pm 0.17$ & $\cdots$ & $\ldots$ & $\ldots$ & $\ldots$ \\
\hline 566 & 2 & $\ldots$ & $\ldots$ & $\ldots$ & $12.24 \pm 0.06$ & $11.92 \pm 0.07$ & $11.68 \pm 0.08$ & $10.87 \pm 0.09$ & -1.35 & $\ldots$ \\
\hline 567 & 2 & $\ldots$ & $\ldots$ & $\ldots$ & $13.84 \pm 0.14$ & $13.32 \pm 0.13$ & $12.95 \pm 0.15$ & $12.26 \pm 0.22$ & -1.09 & $\ldots$ \\
\hline 568 & 2 & $\cdots$ & $\cdots$ & $\cdots$ & $13.23 \pm 0.10$ & $12.53 \pm 0.09$ & $12.00 \pm 0.09$ & $11.23 \pm 0.10$ & -0.59 & $\ldots$ \\
\hline 569 & 2 & $\cdots$ & $\cdots$ & $\cdots$ & $13.24 \pm 0.11$ & $12.78 \pm 0.11$ & $12.29 \pm 0.12$ & $11.68 \pm 0.12$ & -1.08 & $\cdots$ \\
\hline 570 & 3 & $\cdots$ & $\ldots$ & $\ldots$ & $13.55 \pm 0.12$ & $13.11 \pm 0.12$ & $\ldots$ & $\ldots$ & -1.16 & $\cdots$ \\
\hline 574 & 3 & $16.93 \pm 0.16$ & $16.24 \pm 0.18$ & $15.15 \pm 0.16$ & $14.67 \pm 0.20$ & $14.49 \pm 0.22$ & $\cdots$ & $\cdots$ & -2.15 & $\cdots$ \\
\hline 575 & 3 & $10.24 \pm 0.02$ & $9.00 \pm 0.02$ & $7.88 \pm 0.02$ & $6.88 \pm 0.01$ & $6.22 \pm 0.01$ & $5.66 \pm 0.01$ & $5.20 \pm 0.05$ & -0.97 & 0.12 \\
\hline
\end{tabular}


Table 9-Continued

\begin{tabular}{|c|c|c|c|c|c|c|c|c|c|c|}
\hline $\begin{array}{l}S p . \\
\text { ID }\end{array}$ & $\begin{array}{l}\text { Ep. } \\
\text { ID }\end{array}$ & $\begin{array}{c}\mathrm{J} \\
\text { [mag.] }\end{array}$ & $\begin{array}{c}\mathrm{H} \\
{[\mathrm{mag} .]}\end{array}$ & $\begin{array}{c}\mathrm{K} \\
{[\mathrm{mag} .]}\end{array}$ & $\begin{array}{l}3.6 \mu \mathrm{m} \\
{[\mathrm{mag} .]}\end{array}$ & $\begin{array}{l}4.5 \mu \mathrm{m} \\
{[\mathrm{mag} .]}\end{array}$ & $\begin{array}{l}5.8 \mu m \\
{[\mathrm{mag} .]}\end{array}$ & $\begin{array}{l}8.0 \mu m \\
{[\mathrm{mag} .]}\end{array}$ & $\alpha_{I R A C}$ & $\begin{array}{c}A_{K} \\
{[\mathrm{mag} .]}\end{array}$ \\
\hline 579 & 3 & & $15.08 \pm 0.09$ & $14.33 \pm 0.09$ & & $12.90 \pm 0.15$ & . & 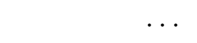 & $\cdots$ & . \\
\hline 582 & 3 & $16.81 \pm 0.20$ & $15.03 \pm 0.09$ & $14.39 \pm 0.12$ & $13.33 \pm 0.55$ & $13.27 \pm 0.54$ & $\ldots$ & $\ldots$ & -2.62 & $\ldots$ \\
\hline 583 & 3 & $\ldots$ & $15.88 \pm 0.20$ & $14.96 \pm 0.18$ & $12.98 \pm 0.20$ & $12.72 \pm 0.24$ & $\ldots$ & $\ldots$ & -1.84 & 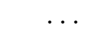 \\
\hline 584 & 3 & $\ldots$ & & $11.54 \pm 0.06$ & $\ldots$ & $9.32 \pm 0.33$ & $\ldots$ & $\ldots$ & $\ldots$ & \\
\hline 587 & 3 & $8.16 \pm 0.02$ & $7.14 \pm 0.04$ & $6.53 \pm 0.02$ & $\ldots$ & $6.26 \pm 0.02$ & $6.04 \pm 0.03$ & $\ldots$ & -2.11 & 0.23 \\
\hline 604 & 2 & $16.67 \pm 0.16$ & $15.58 \pm 0.11$ & $14.78 \pm 0.10$ & $14.24 \pm 0.16$ & $13.80 \pm 0.16$ & $13.34 \pm 0.18$ & $12.64 \pm 0.28$ & -1.03 & . \\
\hline 625 & 2 & $15.97 \pm 0.08$ & $14.38 \pm 0.04$ & $13.72 \pm 0.05$ & $12.86 \pm 0.09$ & $12.46 \pm 0.09$ & $12.01 \pm 0.13$ & $11.17 \pm 0.31$ & -0.93 & 1.04 \\
\hline 641 & 1 & $\ldots$ & $16.30 \pm 0.19$ & $14.98 \pm 0.15$ & $14.32 \pm 0.17$ & $13.88 \pm 0.16$ & $13.41 \pm 0.18$ & $13.00 \pm 0.24$ & -1.34 & $\ldots$ \\
\hline 651 & 4 & $\ldots$ & $14.94 \pm 0.07$ & $13.43 \pm 0.04$ & $12.08 \pm 0.06$ & $11.48 \pm 0.05$ & $11.09 \pm 0.11$ & $10.64 \pm 0.35$ & -1.25 & 1.52 \\
\hline 662 & 3 & $\ldots$ & $14.21 \pm 0.07$ & $12.71 \pm 0.05$ & $11.40 \pm 0.05$ & $10.85 \pm 0.04$ & $10.35 \pm 0.16$ & . & $\ldots$ & $\ldots$ \\
\hline 666 & 4 & $16.22 \pm 0.11$ & $14.51 \pm 0.06$ & $13.66 \pm 0.05$ & $12.73 \pm 0.08$ & $12.42 \pm 0.09$ & $12.15 \pm 0.15$ & . & $\ldots$ & $\ldots$ \\
\hline 670 & 1 & $\cdots$ & $15.60 \pm 0.11$ & $14.65 \pm 0.09$ & $13.68 \pm 0.13$ & $13.16 \pm 0.12$ & $12.60 \pm 0.16$ & $11.75 \pm 0.28$ & $\cdots$ & $\cdots$ \\
\hline 672 & 4 & $15.96 \pm 0.09$ & $14.50 \pm 0.06$ & $13.63 \pm 0.06$ & $13.09 \pm 0.10$ & $12.86 \pm 0.11$ & $12.38 \pm 0.21$ & . & $\ldots$ & $\cdots$ \\
\hline 673 & 4 & $\cdots$ & $\ldots$ & $15.03 \pm 0.15$ & $13.24 \pm 0.10$ & $12.60 \pm 0.09$ & \pm 0.12 & $\ldots$ & $\ldots$ & $\cdots$ \\
\hline 675 & 1 & $\cdots$ & $\cdots$ & $14.43 \pm 0.08$ & $12.99 \pm 0.09$ & $12.60 \pm 0.09$ & $12.15 \pm 0.15$ & $\ldots$ & $\cdots$ & 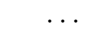 \\
\hline 693 & 4 & $\cdots$ & ... & $14.30 \pm 0.08$ & $12.19 \pm 0.06$ & $11.35 \pm 0.05$ & $10.92 \pm 0.10$ & $10.30 \pm 0.30$ & $\ldots$ & \\
\hline 710 & 4 & . & $13.46 \pm 0.03$ & $10.74 \pm 0.02$ & $8.96 \pm 0.01$ & $8.63 \pm 0.01$ & $8.24 \pm 0.02$ & $8.19 \pm 0.06$ & $\ldots$ & $\cdots$ \\
\hline 757 & 1 & . & $\ldots$ & . & $14.01 \pm 0.15$ & $13.34 \pm 0.13$ & $12.90 \pm 0.15$ & $12.07 \pm 0.20$ & $\cdots$ & $\cdots$ \\
\hline 779 & 4 & $\ldots$ & $\ldots$ & $\ldots$ & $13.47 \pm 0.11$ & $12.89 \pm 0.11$ & $12.34 \pm 0.16$ & $11.94 \pm 0.51$ & $\ldots$ & 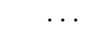 \\
\hline 796 & 4 & $\cdots$ & $15.28 \pm 0.07$ & $12.81 \pm 0.03$ & $10.95 \pm 0.04$ & $10.58 \pm 0.04$ & $10.26 \pm 0.05$ & $10.41 \pm 0.15$ & $\cdots$ & \\
\hline 797 & 4 & $\cdots$ & $\ldots$ & $15.06 \pm 0.15$ & $13.55 \pm 0.12$ & $13.04 \pm 0.11$ & $12.63 \pm 0.16$ & $12.37 \pm 0.37$ & -1.51 & . \\
\hline 798 & 4 & 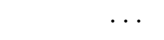 & $12.96 \pm 0.16$ & $11.61 \pm 0.15$ & $10.64 \pm 0.11$ & $9.76 \pm 0.20$ & $9.12 \pm 0.20$ & $7.10 \pm 0.34$ & 1.11 & $\cdots$ \\
\hline 803 & 2 & $10.41 \pm 0.03$ & $8.99 \pm 0.02$ & $8.50 \pm 0.02$ & $8.69 \pm 0.01$ & $8.39 \pm 0.01$ & $8.11 \pm 0.01$ & $8.09 \pm 0.02$ & $\cdots$ & $\cdots$ \\
\hline 804 & 1 & $11.52 \pm 0.02$ & $9.58 \pm 0.02$ & $8.74 \pm 0.02$ & $8.79 \pm 0.01$ & $8.42 \pm 0.01$ & $8.11 \pm 0.02$ & $8.09 \pm 0.02$ & $\cdots$ & $\cdots$ \\
\hline 805 & 4 & $16.47 \pm 0.12$ & $14.46 \pm 0.06$ & $13.41 \pm 0.05$ & $12.64 \pm 0.08$ & $12.25 \pm 0.08$ & $11.76 \pm 0.15$ & $-100.00 \pm 10.00$ & $\cdots$ & \\
\hline 806 & 1 & $11.00 \pm 0.02$ & $9.40 \pm 0.02$ & $8.71 \pm 0.02$ & $8.74 \pm 0.01$ & $8.55 \pm 0.01$ & $8.22 \pm 0.02$ & $8.15 \pm 0.02$ & $\cdots$ & $\cdots$ \\
\hline 807 & 1 & $16.07 \pm 0.09$ & $14.93 \pm 0.06$ & $14.29 \pm 0.07$ & $13.20 \pm 0.10$ & $12.82 \pm 0.10$ & $12.29 \pm 0.10$ & $10.61 \pm 0.07$ & $\cdots$ & \\
\hline 808 & 2 & $10.46 \pm 0.02$ & $8.98 \pm 0.03$ & $8.31 \pm 0.03$ & $8.72 \pm 0.01$ & $8.28 \pm 0.01$ & $7.90 \pm 0.01$ & $7.93 \pm 0.03$ & $\ldots$ & . \\
\hline 809 & 1 & $10.35 \pm 0.02$ & $8.80 \pm 0.04$ & $8.19 \pm 0.02$ & $8.90 \pm 0.01$ & $8.12 \pm 0.01$ & $7.75 \pm 0.01$ & $7.68 \pm 0.02$ & $\cdots$ & $\cdots$ \\
\hline 810 & 4 & $\ldots$ & $\cdots$ & $\cdots$ & $7.78 \pm 0.01$ & $7.73 \pm 0.01$ & $7.69 \pm 0.01$ & $7.66 \pm 0.02$ & $\cdots$ & $\cdots$ \\
\hline 811 & 4 & $9.12 \pm 0.03$ & $7.46 \pm 0.05$ & 0.03 & $6.59 \pm 0.01$ & $6.39 \pm 0.01$ & $6.02 \pm 0.01$ & $5.88 \pm 0.01$ & $\ldots$ & 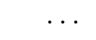 \\
\hline 812 & 4 & $14.11 \pm 0.03$ & $11.05 \pm 0.02$ & $9.66 \pm 0.02$ & $8.72 \pm 0.01$ & $8.66 \pm 0.01$ & $8.39 \pm 0.02$ & $8.28 \pm 0.04$ & $\ldots$ & $\ldots$ \\
\hline
\end{tabular}


Table 10. Photometry of the Class III YSOs in RCW 38.

\begin{tabular}{|c|c|c|c|c|c|c|c|c|c|c|}
\hline $\begin{array}{l}S p . \\
\text { ID }\end{array}$ & $\begin{array}{c}\text { Ep. } \\
\text { ID }\end{array}$ & $\begin{array}{c}\mathrm{J} \\
\text { [mag.] }\end{array}$ & $\begin{array}{c}\mathrm{H} \\
{[\mathrm{mag} .]}\end{array}$ & $\begin{array}{c}\mathrm{K} \\
{[\mathrm{mag} .]}\end{array}$ & $\begin{array}{l}3.6 \mu \mathrm{m} \\
{[\mathrm{mag} .]}\end{array}$ & $\begin{array}{l}4.5 \mu \mathrm{m} \\
\text { [mag.] }\end{array}$ & $\begin{array}{l}5.8 \mu \mathrm{m} \\
\text { [mag.] }\end{array}$ & $\begin{array}{l}8.0 \mu \mathrm{m} \\
{[\mathrm{mag} .]}\end{array}$ & $\alpha_{I R A C}$ & $\begin{array}{c}A_{K} \\
{[\mathrm{mag} .]}\end{array}$ \\
\hline 17 & 3 & $15.85 \pm 0.10$ & $14.71 \pm 0.07$ & $14.20 \pm 0.08$ & $13.83 \pm 0.14$ & $13.72 \pm 0.15$ & $\ldots$ & $\cdots$ & -2.67 & 0.52 \\
\hline 36 & 3 & $14.94 \pm 0.04$ & $13.67 \pm 0.03$ & $13.38 \pm 0.03$ & $12.83 \pm 0.10$ & $13.17 \pm 0.24$ & $\ldots$ & $\ldots$ & -4.48 & 0.71 \\
\hline 65 & 3 & $12.22 \pm 0.02$ & $11.97 \pm 0.03$ & $11.76 \pm 0.02$ & $11.75 \pm 0.06$ & $11.70 \pm 0.07$ & $\ldots$ & $\ldots$ & -2.66 & $\ldots$ \\
\hline 77 & 3 & $14.19 \pm 0.03$ & $13.42 \pm 0.03$ & $13.04 \pm 0.03$ & $12.74 \pm 0.08$ & $12.69 \pm 0.09$ & $12.68 \pm 0.15$ & $\ldots$ & -2.78 & 0.05 \\
\hline 82 & 3 & $11.64 \pm 0.02$ & $11.39 \pm 0.03$ & $11.34 \pm 0.03$ & $11.37 \pm 0.04$ & $11.37 \pm 0.05$ & $11.47 \pm 0.08$ & $11.98 \pm 0.55$ & -3.61 & $\ldots$ \\
\hline 93 & 1 & $15.31 \pm 0.07$ & $14.33 \pm 0.09$ & $13.87 \pm 0.07$ & $\ldots$ & $13.65 \pm 0.25$ & $\ldots$ & $\ldots$ & $\ldots$ & 0.32 \\
\hline 99 & 3 & $16.56 \pm 0.16$ & $14.88 \pm 0.06$ & $14.05 \pm 0.07$ & $13.19 \pm 0.12$ & $13.27 \pm 0.14$ & $\ldots$ & $\ldots$ & -3.19 & $\ldots$ \\
\hline 100 & 1 & $15.21 \pm 0.05$ & $14.18 \pm 0.04$ & $13.81 \pm 0.06$ & $13.57 \pm 0.12$ & $\ldots$ & $13.53 \pm 0.27$ & $\cdots$ & -2.94 & 0.45 \\
\hline 109 & 3 & $14.08 \pm 0.03$ & $13.45 \pm 0.03$ & $13.35 \pm 0.05$ & $13.08 \pm 0.13$ & $13.15 \pm 0.15$ & $\ldots$ & $\ldots$ & -3.15 & 0.04 \\
\hline 123 & 3 & .. & $14.80 \pm 0.06$ & $13.84 \pm 0.05$ & $13.31 \pm 0.31$ & $13.19 \pm 0.31$ & $\ldots$ & $\cdots$ & -2.39 & $\ldots$ \\
\hline 125 & 3 & . & $15.42 \pm 0.12$ & $\ldots$ & & $13.14 \pm 0.20$ & $\ldots$ & $\ldots$ & $\cdots$ & $\ldots$ \\
\hline 129 & 4 & $8.59 \pm 0.02$ & $8.25 \pm 0.04$ & $8.07 \pm 0.02$ & $8.00 \pm 0.01$ & $\ldots$ & $7.98 \pm 0.01$ & $\ldots$ & -2.84 & $\ldots$ \\
\hline 133 & 3 & & $14.15 \pm 0.05$ & $13.13 \pm 0.04$ & $12.22 \pm 0.09$ & $12.05 \pm 0.10$ & $\ldots$ & $\ldots$ & -2.78 & 1.50 \\
\hline 166 & 3 & $15.77 \pm 0.08$ & $14.57 \pm 0.07$ & $13.85 \pm 0.07$ & $12.93 \pm 0.16$ & $13.17 \pm 0.20$ & $\ldots$ & $\ldots$ & -3.96 & 0.41 \\
\hline 169 & 3 & $16.52 \pm 0.14$ & $15.10 \pm 0.08$ & $14.56 \pm 0.09$ & $14.13 \pm 0.16$ & $14.05 \pm 0.18$ & $\ldots$ & $\ldots$ & -2.53 & $\ldots$ \\
\hline 190 & 3 & $15.91 \pm 0.10$ & $14.10 \pm 0.05$ & $13.20 \pm 0.06$ & $12.58 \pm 0.29$ & $12.52 \pm 0.27$ & $\ldots$ & $\ldots$ & -3.08 & 1.22 \\
\hline 194 & 3 & $\cdots$ & $9.95 \pm 0.05$ & $9.26 \pm 0.03$ & $9.02 \pm 0.07$ & $\ldots$ & $\ldots$ & $\cdots$ & $\cdots$ & 0.88 \\
\hline 195 & 3 & $15.58 \pm 0.07$ & $14.27 \pm 0.04$ & $13.74 \pm 0.05$ & $13.54 \pm 0.12$ & $13.52 \pm 0.14$ & $\ldots$ & $\ldots$ & -3.11 & 0.74 \\
\hline 210 & 3 & $12.15 \pm 0.03$ & $11.66 \pm 0.03$ & $11.39 \pm 0.03$ & $11.25 \pm 0.04$ & $11.20 \pm 0.05$ & $11.21 \pm 0.09$ & $11.18 \pm 0.33$ & -2.83 & $\cdots$ \\
\hline 221 & 3 & $15.88 \pm 0.08$ & $14.37 \pm 0.05$ & $13.68 \pm 0.05$ & $13.37 \pm 0.13$ & $13.29 \pm 0.14$ & $\ldots$ & $\ldots$ & -2.92 & 0.93 \\
\hline 225 & 3 & $13.86 \pm 0.04$ & $13.13 \pm 0.04$ & $13.01 \pm 0.05$ & $12.91 \pm 0.10$ & $12.88 \pm 0.12$ & $\ldots$ & $\ldots$ & -2.80 & 0.13 \\
\hline 229 & 3 & $16.28 \pm 0.12$ & $14.74 \pm 0.07$ & $14.07 \pm 0.06$ & $13.52 \pm 0.13$ & $13.52 \pm 0.17$ & $\ldots$ & $\ldots$ & -2.84 & $\ldots$ \\
\hline 243 & 3 & $14.66 \pm 0.02$ & $12.71 \pm 0.02$ & $11.83 \pm 0.02$ & $11.14 \pm 0.12$ & $10.96 \pm 0.14$ & $\ldots$ & $\ldots$ & -2.71 & 1.42 \\
\hline 255 & 3 & $14.28 \pm 0.03$ & $12.41 \pm 0.03$ & $11.51 \pm 0.03$ & $10.80 \pm 0.10$ & $10.66 \pm 0.08$ & $\ldots$ & $\ldots$ & -2.83 & 1.31 \\
\hline 262 & 1 & $16.43 \pm 0.14$ & $15.07 \pm 0.08$ & $14.62 \pm 0.09$ & $14.29 \pm 0.18$ & $14.25 \pm 0.21$ & $\ldots$ & $\ldots$ & -2.69 & $\ldots$ \\
\hline 263 & 3 & $16.18 \pm 0.10$ & $14.81 \pm 0.07$ & $14.20 \pm 0.08$ & $13.87 \pm 0.24$ & $13.77 \pm 0.24$ & $\ldots$ & $\cdots$ & -2.77 & 0.79 \\
\hline 278 & 3 & $15.93 \pm 0.09$ & $15.25 \pm 0.10$ & $15.16 \pm 0.20$ & $14.76 \pm 0.22$ & $14.78 \pm 0.26$ & $\ldots$ & $\ldots$ & -2.94 & $\ldots$ \\
\hline 280 & 3 & $15.46 \pm 0.06$ & $14.16 \pm 0.03$ & $13.56 \pm 0.03$ & $12.91 \pm 0.13$ & $12.92 \pm 0.19$ & $\cdots$ & $\cdots$ & -3.18 & 0.68 \\
\hline 281 & 3 & $12.30 \pm 0.06$ & $10.88 \pm 0.04$ & $10.24 \pm 0.05$ & $9.40 \pm 0.14$ & $9.62 \pm 0.39$ & $\ldots$ & $\ldots$ & -4.02 & 0.84 \\
\hline 290 & 3 & $\cdots$ & $13.65 \pm 0.04$ & $12.76 \pm 0.04$ & $12.22 \pm 0.08$ & $12.16 \pm 0.13$ & $\ldots$ & $\cdots$ & -3.13 & 1.26 \\
\hline 295 & 3 & $15.56 \pm 0.07$ & $14.03 \pm 0.06$ & $13.25 \pm 0.05$ & $12.96 \pm 0.10$ & $12.83 \pm 0.11$ & $12.81 \pm 0.54$ & $\ldots$ & -2.84 & 0.87 \\
\hline 297 & 3 & $14.75 \pm 0.04$ & $13.45 \pm 0.05$ & $12.82 \pm 0.04$ & $12.53 \pm 0.07$ & $12.48 \pm 0.09$ & $\cdots$ & $\cdots$ & -2.92 & 0.65 \\
\hline 302 & 3 & $16.77 \pm 0.18$ & $15.70 \pm 0.13$ & $15.04 \pm 0.14$ & $\cdots$ & $13.97 \pm 0.49$ & $\cdots$ & $\ldots$ & $\ldots$ & $\ldots$ \\
\hline 314 & 3 & $14.05 \pm 0.03$ & $13.39 \pm 0.03$ & $13.25 \pm 0.04$ & $13.16 \pm 0.10$ & $13.06 \pm 0.12$ & $13.22 \pm 0.62$ & $\ldots$ & -3.02 & 0.06 \\
\hline 317 & 3 & $11.14 \pm 0.02$ & $10.33 \pm 0.02$ & $9.89 \pm 0.02$ & $9.44 \pm 0.10$ & $9.53 \pm 0.24$ & $\ldots$ & $\ldots$ & -3.24 & 0.06 \\
\hline 318 & 4 & $13.59 \pm 0.03$ & $12.38 \pm 0.03$ & $11.74 \pm 0.03$ & $\ldots$ & $11.25 \pm 0.05$ & $\ldots$ & $11.17 \pm 0.16$ & -2.80 & 0.50 \\
\hline 320 & 3 & $16.31 \pm 0.12$ & $14.77 \pm 0.08$ & $13.85 \pm 0.07$ & $13.38 \pm 0.12$ & $13.29 \pm 0.13$ & $\ldots$ & $\ldots$ & -2.50 & $\ldots$ \\
\hline 327 & 3 & $15.35 \pm 0.06$ & $14.04 \pm 0.04$ & $13.52 \pm 0.04$ & $13.05 \pm 0.10$ & $12.96 \pm 0.14$ & $\ldots$ & $\ldots$ & -2.78 & 0.75 \\
\hline 334 & 3 & $14.64 \pm 0.04$ & $13.30 \pm 0.03$ & $12.66 \pm 0.04$ & $12.43 \pm 0.08$ & $12.36 \pm 0.09$ & $12.25 \pm 0.42$ & $\ldots$ & -2.73 & 0.71 \\
\hline 337 & 3 & $11.51 \pm 0.07$ & $10.52 \pm 0.08$ & $9.32 \pm 0.06$ & $9.76 \pm 0.13$ & $9.22 \pm 0.20$ & $\ldots$ & $\ldots$ & -0.76 & $\ldots$ \\
\hline 345 & 3 & $15.83 \pm 0.06$ & $14.61 \pm 0.06$ & $14.01 \pm 0.07$ & $13.75 \pm 0.21$ & $13.62 \pm 0.19$ & $\cdots$ & $\ldots$ & -2.55 & 0.55 \\
\hline 354 & 4 & . & $15.22 \pm 0.08$ & $14.21 \pm 0.07$ & $13.07 \pm 0.25$ & $13.03 \pm 0.26$ & $\ldots$ & $\ldots$ & -2.67 & $\ldots$ \\
\hline 358 & 3 & $16.08 \pm 0.09$ & $15.09 \pm 0.07$ & $14.51 \pm 0.09$ & $14.16 \pm 0.26$ & $14.19 \pm 0.30$ & $\ldots$ & $\ldots$ & -3.06 & 0.20 \\
\hline 366 & 3 & $16.27 \pm 0.11$ & $15.28 \pm 0.09$ & $14.87 \pm 0.13$ & $14.44 \pm 0.21$ & $14.77 \pm 0.33$ & $\ldots$ & $\cdots$ & -4.15 & $\ldots$ \\
\hline 372 & 3 & $16.34 \pm 0.12$ & $14.65 \pm 0.06$ & $14.02 \pm 0.07$ & $13.60 \pm 0.23$ & $13.54 \pm 0.25$ & $\ldots$ & $\ldots$ & -2.66 & $\cdots$ \\
\hline
\end{tabular}


Table 10-Continued

\begin{tabular}{|c|c|c|c|c|c|c|c|c|c|c|}
\hline $\begin{array}{l}S p . \\
\text { ID }\end{array}$ & $\begin{array}{l}\text { Ep. } \\
\text { ID }\end{array}$ & $\begin{array}{c}\mathrm{J} \\
\text { [mag.] }\end{array}$ & $\begin{array}{c}\mathrm{H} \\
{[\mathrm{mag} .]}\end{array}$ & $\begin{array}{c}\mathrm{K} \\
{[\mathrm{mag} .]}\end{array}$ & $\begin{array}{l}3.6 \mu \mathrm{m} \\
{[\mathrm{mag} .]}\end{array}$ & $\begin{array}{l}4.5 \mu \mathrm{m} \\
{[\mathrm{mag} .]}\end{array}$ & $\begin{array}{l}5.8 \mu \mathrm{m} \\
{[\mathrm{mag} .]}\end{array}$ & $\begin{array}{l}8.0 \mu \mathrm{m} \\
{[\mathrm{mag} .]}\end{array}$ & $\alpha_{I R A C}$ & $\begin{array}{c}A_{K} \\
\text { [mag.] }\end{array}$ \\
\hline 373 & 3 & $14.34 \pm 0.03$ & $13.07 \pm 0.04$ & $12.40 \pm 0.04$ & $11.65 \pm 0.19$ & $11.69 \pm 0.32$ & $\cdots$ & $\cdots$ & -3.25 & 0.58 \\
\hline 375 & 3 & $15.69 \pm 0.09$ & $14.36 \pm 0.05$ & $13.75 \pm 0.06$ & $13.57 \pm 0.13$ & $13.53 \pm 0.16$ & $\cdots$ & $\cdots$ & -3.01 & 0.72 \\
\hline 380 & 4 & $17.16 \pm 0.21$ & $15.81 \pm 0.13$ & $15.48 \pm 0.22$ & $14.78 \pm 0.22$ & $14.93 \pm 0.31$ & $\ldots$ & $\ldots$ & -3.43 & $\cdots$ \\
\hline 398 & 3 & $15.69 \pm 0.09$ & $14.31 \pm 0.05$ & $13.62 \pm 0.05$ & $13.22 \pm 0.12$ & $13.18 \pm 0.15$ & $\ldots$ & $\ldots$ & -2.97 & 0.72 \\
\hline 400 & 3 & $13.15 \pm 0.02$ & $11.74 \pm 0.02$ & $11.14 \pm 0.02$ & $10.76 \pm 0.04$ & $10.76 \pm 0.05$ & $10.41 \pm 0.24$ & $\cdots$ & -2.43 & 0.86 \\
\hline 427 & 3 & $13.24 \pm 0.03$ & $12.68 \pm 0.03$ & $12.59 \pm 0.03$ & $12.65 \pm 0.08$ & $12.57 \pm 0.09$ & $12.52 \pm 0.15$ & $\ldots$ & -2.63 & $\ldots$ \\
\hline 437 & 4 & $10.95 \pm 0.02$ & $10.55 \pm 0.02$ & $10.46 \pm 0.02$ & $10.48 \pm 0.03$ & $\cdots$ & $10.48 \pm 0.10$ & $\ldots$ & -2.89 & $\cdots$ \\
\hline 439 & 4 & $14.90 \pm 0.05$ & $14.19 \pm 0.04$ & $14.06 \pm 0.08$ & $13.86 \pm 0.14$ & $\cdots$ & $\cdots$ & $\cdots$ & $\cdots$ & 0.12 \\
\hline 440 & 3 & $10.93 \pm 0.02$ & $9.87 \pm 0.03$ & $9.41 \pm 0.02$ & $9.14 \pm 0.02$ & $9.09 \pm 0.02$ & $9.06 \pm 0.02$ & $9.03 \pm 0.04$ & -2.83 & 0.42 \\
\hline 447 & 3 & $14.34 \pm 0.03$ & $13.42 \pm 0.03$ & $13.00 \pm 0.03$ & $12.70 \pm 0.08$ & $12.61 \pm 0.09$ & $12.54 \pm 0.16$ & $\ldots$ & -2.63 & 0.25 \\
\hline 454 & 3 & $15.44 \pm 0.06$ & $14.69 \pm 0.06$ & $14.65 \pm 0.11$ & $13.94 \pm 0.15$ & $13.86 \pm 0.17$ & $\cdots$ & $\ldots$ & -2.55 & $\ldots$ \\
\hline 455 & 3 & $15.42 \pm 0.06$ & $14.82 \pm 0.06$ & $14.64 \pm 0.09$ & $14.44 \pm 0.18$ & $14.45 \pm 0.22$ & $\cdots$ & $\cdots$ & -2.93 & $\cdots$ \\
\hline 458 & 3 & $15.65 \pm 0.08$ & $14.37 \pm 0.07$ & $13.75 \pm 0.07$ & $13.65 \pm 0.13$ & $13.64 \pm 0.15$ & $\ldots$ & $\ldots$ & -3.07 & 0.62 \\
\hline 463 & 4 & $15.49 \pm 0.05$ & $14.41 \pm 0.05$ & $13.89 \pm 0.06$ & $13.62 \pm 0.12$ & $\ldots$ & $13.70 \pm 0.37$ & $\cdots$ & -3.14 & 0.40 \\
\hline 468 & 3 & $\cdots$ & $\cdots$ & $\ldots$ & $13.59 \pm 0.32$ & $13.62 \pm 0.32$ & $\cdots$ & $\ldots$ & -2.97 & $\ldots$ \\
\hline 482 & 3 & $\cdots$ & $\cdots$ & $\cdots$ & $14.47 \pm 0.25$ & $14.56 \pm 0.28$ & $\cdots$ & $\cdots$ & -3.21 & $\cdots$ \\
\hline 484 & 3 & $\cdots$ & $\cdots$ & $\cdots$ & $14.96 \pm 0.23$ & $14.96 \pm 0.28$ & $\cdots$ & $\cdots$ & -2.85 & $\cdots$ \\
\hline 511 & 3 & $\cdots$ & $14.55 \pm 0.06$ & $13.90 \pm 0.07$ & $13.59 \pm 0.16$ & $13.68 \pm 0.22$ & $\cdots$ & $\ldots$ & -3.23 & $\cdots$ \\
\hline 517 & 3 & $15.64 \pm 0.08$ & $14.31 \pm 0.04$ & $13.93 \pm 0.06$ & $13.26 \pm 0.13$ & $13.13 \pm 0.17$ & $\ldots$ & $\ldots$ & -2.65 & 0.77 \\
\hline 553 & 3 & $17.07 \pm 0.22$ & $15.48 \pm 0.10$ & $14.37 \pm 0.08$ & $13.41 \pm 0.13$ & $13.39 \pm 0.18$ & $\ldots$ & $\cdots$ & -2.78 & $\cdots$ \\
\hline 554 & 2 & $\cdots$ & $15.00 \pm 0.07$ & $14.00 \pm 0.06$ & $13.83 \pm 0.30$ & $\cdots$ & $\cdots$ & $\cdots$ & $\cdots$ & $\cdots$ \\
\hline 555 & 3 & $\ldots$ & $14.20 \pm 0.17$ & $12.57 \pm 0.15$ & $\ldots$ & $10.70 \pm 0.44$ & $\ldots$ & $\ldots$ & $\ldots$ & $\ldots$ \\
\hline 562 & 4 & $16.81 \pm 0.17$ & $16.00 \pm 0.19$ & $15.09 \pm 0.18$ & $15.09 \pm 0.28$ & $15.09 \pm 0.32$ & $\cdots$ & $\cdots$ & -2.86 & $\cdots$ \\
\hline 576 & 3 & $15.34 \pm 0.06$ & $14.41 \pm 0.05$ & $14.10 \pm 0.09$ & $\ldots$ & $13.98 \pm 0.32$ & $\ldots$ & $\ldots$ & $\ldots$ & 0.35 \\
\hline 578 & 3 & $\cdots$ & $15.31 \pm 0.10$ & $14.43 \pm 0.11$ & $\cdots$ & $13.95 \pm 0.67$ & $\cdots$ & $\cdots$ & $\cdots$ & $\cdots$ \\
\hline 580 & 3 & $16.50 \pm 0.14$ & $14.87 \pm 0.08$ & $\ldots$ & $12.76 \pm 0.32$ & $\ldots$ & $\ldots$ & $\ldots$ & $\cdots$ & $\ldots$ \\
\hline 586 & 3 & $14.55 \pm 0.19$ & $12.99 \pm 0.12$ & $11.96 \pm 0.14$ & $11.37 \pm 0.41$ & $\ldots$ & $\ldots$ & $\cdots$ & $\cdots$ & $\ldots$ \\
\hline 588 & 3 & $15.47 \pm 0.06$ & $14.79 \pm 0.05$ & $14.61 \pm 0.11$ & $14.51 \pm 0.73$ & $\cdots$ & $\cdots$ & $\ldots$ & $\cdots$ & $\ldots$ \\
\hline 591 & 4 & $\ldots$ & $14.93 \pm 0.37$ & $14.51 \pm 0.19$ & & $14.14 \pm 0.28$ & $\cdots$ & $\ldots$ & $\cdots$ & $\ldots$ \\
\hline
\end{tabular}


Table 11. Photometry of the Candidate [3.6] v. [3.6-5.8] YSOs in RCW 38.

\begin{tabular}{|c|c|c|c|c|c|c|c|c|c|c|}
\hline $\begin{array}{l}S p . \\
\text { ID }\end{array}$ & $\begin{array}{l}\text { Ep. } \\
\text { ID }\end{array}$ & $\begin{array}{c}\text { RA } \\
\text { J2000 }\end{array}$ & $\begin{array}{c}\text { Dec } \\
\text { J2000 }\end{array}$ & $\begin{array}{c}\mathrm{J} \\
\text { [mag.] }\end{array}$ & $\begin{array}{c}\mathrm{H} \\
\text { [mag.] }\end{array}$ & $\begin{array}{c}\mathrm{K} \\
\text { [mag.] }\end{array}$ & $\begin{array}{l}3.6 \mu \mathrm{m} \\
\text { [mag.] }\end{array}$ & $\begin{array}{l}4.5 \mu \mathrm{m} \\
\text { [mag.] }\end{array}$ & $\begin{array}{l}5.8 \mu \mathrm{m} \\
\text { [mag.] }\end{array}$ & $\begin{array}{l}8.0 \mu \mathrm{m} \\
\text { [mag.] }\end{array}$ \\
\hline 605 & 4 & & & & & & & & & \\
\hline & & & & & & & & & & \\
\hline 607 & 4 & & $-47: 36: 43.69$ & $.72 \pm 0.15$ & $.73 \pm 0.06$ & $14.18 \pm 0.07$ & $13.52 \pm 0.12$ & 0.13 & 19 & \\
\hline 609 & 4 & 8:59: & $-47: 37: 8.86$ & $65 \pm 0.17$ & 0.11 & $14.76 \pm 0.11$ & $13.82 \pm 0.14$ & $13.53 \pm 0.14$ & $=0.22$ & \pm 0.35 \\
\hline 610 & 4 & $: 27.16$ & $-47: 34: 4$ & & $14.83 \pm$ & $14.41=$ & & & & \\
\hline 611 & 2 & & $-47: 41: 39$ & $15.59 \pm$ & $14.14=$ & $13.57 \pm$ & 13.16 & 13.17 & & \\
\hline 612 & 4 & 20 & $-47: 36: 1$ & 0.17 & 07 & $14.16=$ & 0.12 & .13 & .12 & \\
\hline 613 & 4 & $58: 22.97$ & $-47: 34: 43.75$ & $\ldots$ & 0.05 & $12.92 \pm 0.04$ & 12.54 & 12.47 & & \\
\hline 614 & 4 & $8: 58: 33.15$ & $-47: 38: 3.43$ & $\cdots$ & $16 \pm 0.18$ & $14.76 \pm 0.13$ & $13.81 \pm 0.14$ & 13.26 & 13.07 & 0.34 \\
\hline 615 & 4 & $8: 59: 18.39$ & $-47: 35: 26.73$ & $14.28 \pm 0.04$ & $12.49 \pm 0$ & $11.77 \pm$ & 11.29 & 11.23 & 10.7 & \\
\hline 616 & 4 & & $-47: 34: 4$ & & & & & & & . \\
\hline 617 & 4 & & $-47: 3$ & 0.03 & & 11.7 & & & & \pm 0.22 \\
\hline 618 & 4 & & $-47: 35: 3$ & $15.10 \pm 0.05$ & 12.9 & $12.01=$ & & & 10.2 & \\
\hline 619 & 2 & 3 & $-47:$ & & 15 & & & & & $=0.43$ \\
\hline 620 & 2 & 7 & $-47: 39: 2$ & 15 & 14.9 & 14.39 & 13. & 13. & & \\
\hline 621 & 3 & 7 & $-47: 36:$ & 15.82 & 14.0 & 13.0 & & 12. & & \\
\hline 622 & 2 & 9 & $-47: 36$ & $15.88 \pm 0.07$ & 14.7 & 07 & & & & \\
\hline 623 & 4 & & $-47: 3$ & & & & & & & . \\
\hline 624 & 4 & $\varepsilon$ & $-47: 3$ & 13 & 12.7 & 12. & 12 . & 12 . & & \\
\hline 626 & 2 & & -47 & & & & & & & 0.15 \\
\hline 627 & 4 & 3 & $-47: 3$ & 15.6 & 14.3 & 13. & & & 12 . & \\
\hline 628 & 2 & & $-47: 4$ & 16.9 & 15.4 & 14. & 14. & & & \\
\hline 629 & 4 & rat & $-47: 3$ & $16.82 \pm 0.17$ & 14.5 & & & 12. & & \\
\hline 63 & 4 & & $-47: 3$ & & & & & & & \\
\hline 631 & 4 & 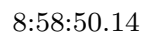 & $-47: 3$ & . & 15.8 & 14.6 & 13. & 13. & 12 . & 0.31 \\
\hline 632 & 3 & 72 & $-47:$ & & 14.8 & 13.8 & & & .16 & $=0.30$ \\
\hline 633 & 2 & $8: 59$ & $-47: 39: 19.30$ & $16.84 \pm 0.19$ & 15.3 & 14.6 & 13. & & & \pm 0.12 \\
\hline 634 & 4 & & $-47: 3$ & 16.0 & & & & & & \\
\hline 635 & 1 & & $-47:$ & 15 . & & 12 & 12 . & & 11. & $=0.18$ \\
\hline 636 & 4 & & $-47:$ & 15 . & & & & & & \\
\hline 637 & 1 & .70 & $-47: 35: 09.27$ & $14.35 \pm 0.04$ & & $13.73 \pm 0.05$ & & & & \\
\hline 638 & 2 & & $-47: 24: 24$ & & 13. & $13.72 \pm 0.05$ & & & & \\
\hline 63 & 1 & & $-47:$ & & & & & & & \\
\hline 640 & 1 & $\varepsilon$ & $-47: 3$ & 16 . & 15 . & 14 & 14 & 14 & 13 & \\
\hline 642 & 1 & & $-47: 18: 24.80$ & $15.07 \pm 0.05$ & $13.52 \pm 0.03$ & $12.99 \pm 0.03$ & $12.60 \pm$ & 12.5 & & \\
\hline 643 & 4 & 8:59: & $-47: 29: 4$ & $15.19 \pm 0.06$ & 13.04 & 12.08 & 11. & 11. & 10 & \\
\hline 644 & 1 & & -47 & & & & & & & \\
\hline 645 & 1 & .22 & $-47: 2$ & $13.32 \pm 0.03$ & 12.40 & 12.03 & 11. & 11.2 & $=0.06$ & 0.20 \\
\hline 646 & 2 & $8: 58$ & $-47: 22: 54.97$ & $13.84 \pm 0.03$ & $13.34 \pm 0.03$ & $13.10 \pm 0.04$ & 13.0 & 12.97 & \pm 0.14 & \\
\hline 647 & 4 & 0.51 & $-47: 29: 8.46$ & $15.45 \pm 0.06$ & 13.89 & 13.17 & 12.5 & 12.5 & 11. & \\
\hline 648 & 1 & $8: 5$ & $-47: 17: 14.87$ & $14.70 \pm 0.06$ & $14.02=$ & 13.91 & 13.6 & 13.5 & 12 & \\
\hline 649 & 1 & & $-47: 2$ & & & & & & & \\
\hline 650 & 1 & 1.56 & $-47: 25: 55.79$ & $16.21 \pm 0.11$ & $14.79 \pm 0.08$ & $14.16 \pm 0.09$ & $13.53 \pm 0.12$ & $13.17 \pm 0.12$ & $12.80 \pm 0.16$ & $12.08 \pm 0.29$ \\
\hline 652 & 2 & $8: 58: 18.05$ & $-47: 30: 22.96$ & & $15.45 \pm 0.10$ & $13.93 \pm 0.05$ & $13.15 \pm 0.10$ & $12.96 \pm 0.11$ & $12.71 \pm 0.16$ & $11.59 \pm 0.17$ \\
\hline 653 & 1 & $8: 58: 43.45$ & $-47: 18: 19.13$ & $13.14 \pm 0.02$ & $12.61 \pm 0.03$ & $12.24 \pm 0.03$ & $12.11 \pm 0.06$ & $12.03 \pm 0.07$ & $11.50 \pm 0.10$ & $10.44 \pm 0.14$ \\
\hline
\end{tabular}


Table 11-Continued

\begin{tabular}{|c|c|c|c|c|c|c|c|c|c|c|}
\hline $\begin{array}{l}S p . \\
\text { ID }\end{array}$ & $\begin{array}{c}\text { Ep. } \\
\text { ID }\end{array}$ & $\begin{array}{c}\text { RA } \\
\text { J2000 }\end{array}$ & $\begin{array}{c}\text { Dec } \\
\text { J2000 }\end{array}$ & $\begin{array}{c}\mathrm{J} \\
{[\mathrm{mag} .]}\end{array}$ & $\begin{array}{c}\mathrm{H} \\
{[\mathrm{mag} .]}\end{array}$ & $\begin{array}{c}\mathrm{K} \\
{[\mathrm{mag} .]}\end{array}$ & $\begin{array}{l}3.6 \mu \mathrm{m} \\
\text { [mag.] }\end{array}$ & $\begin{array}{l}4.5 \mu \mathrm{m} \\
\text { [mag.] }\end{array}$ & $\begin{array}{l}5.8 \mu \mathrm{m} \\
\text { [mag.] }\end{array}$ & $\begin{array}{l}8.0 \mu \mathrm{m} \\
\text { [mag.] }\end{array}$ \\
\hline 654 & 4 & .65 & $-47: 25$ & $14.31 \pm 0.03$ & 0.02 & 13.5 & 13.2 & 13. & 12. & \\
\hline 655 & 4 & $8: 58: 2$ & $-47: 2$ & $17.12 \pm 0.20$ & $14.14 \pm 0.05$ & & & & & \\
\hline 656 & 4 & 8:59:18.19 & $-47: 30: 20.83$ & & & $14.78 \pm 0.13$ & $12.04 \pm 0.07$ & $11.32 \pm 0.06$ & $9.88 \pm 0.08$ & .. \\
\hline 657 & 4 & 8:59:37.70 & $-47: 33: 57.50$ & $15.32 \pm 0.06$ & $14.12 \pm 0.04$ & $13.46 \pm 0.06$ & $12.76 \pm 0.08$ & $12.55 \pm 0.09$ & $12.42 \pm 0.18$ & \\
\hline 658 & 1 & 8:58:18.86 & $-47: 15: 19.57$ & $15.77 \pm 0.08$ & $14.93 \pm 0.07$ & $14.30 \pm 0.08$ & $13.98 \pm 0.15$ & $13.85 \pm 0.16$ & $12.97 \pm 0.19$ & \\
\hline 659 & 1 & $8: 58: 4.58$ & $-47: 25: 5.91$ & & $14.94 \pm 0.14$ & & $13.00 \pm 0.09$ & $12.53=$ & 12.23 & $12.09 \pm 0.29$ \\
\hline 660 & 2 & 8:58:16.90 & $-47: 32: 13.77$ & $15.29 \pm 0.05$ & $14.34 \pm 0.05$ & $14.11 \pm 0.05$ & $14.09 \pm 0.15$ & $14.17 \pm 0.19$ & $12.96 \pm 0.19$ & $\ldots$ \\
\hline 661 & 4 & 8:58:44.27 & $-47: 25: 6.50$ & $\ldots$ & $14.81 \pm 0.06$ & $13.43 \pm 0.04$ & $12.54 \pm 0.08$ & 12.40 & 11.38 & $\ldots$ \\
\hline 663 & 2 & 8:58:12.88 & $-47: 27: 24.48$ & & $15.37 \pm 0.09$ & $14.68 \pm 0.11$ & $13.93 \pm 0.14$ & 13.84 & 12.98 & \\
\hline 664 & 1 & 8:58:06.52 & $-47: 23: 52.66$ & & $14.60 \exists$ & 13.7 & 12.97 & 12.53 & 11.92 & $=0.26$ \\
\hline 665 & 4 & $8: 58: 2$ & $-47: 24: 49.27$ & $14.63 \pm 0.03$ & $13.83 \pm 0.03$ & $13.46 \pm 0.04$ & $13.26 \pm 0.10$ & 13.18 & 13.0 & \\
\hline 667 & 3 & $8: 58: 3$ & $-47: 29: 0.77$ & $16.15 \pm 0.08$ & $14.34 \pm 0.03$ & 13.56 & 12.77 & 12.56 & 11. & $\ldots$ \\
\hline 668 & 4 & 8:58:28.29 & $-47: 31: 20.53$ & $16.07 \pm 0.08$ & $13.54 \pm 0.02$ & $12.34 \pm 0.03$ & $11.59 \pm 0.05$ & 11.44 & 10.9 & \\
\hline 669 & 1 & 8:58:7.87 & $-47: 27: 20.48$ & & $15.93 \pm 0.15$ & $15.12 \pm 0.17$ & $14.10 \pm 0.15$ & 13.64 & 13.30 & $=0.20$ \\
\hline 671 & 4 & $8: 58:$ & $-47: 25: 25.68$ & $16.77 \pm 0.16$ & $14.90 \pm 0.07$ & 13.8 & 12.87 & 12.5 & 11.4 & \\
\hline 674 & 2 & $8: 5$ & $-47: 2$ & $\ldots$ & 15.7 & 14. & 14. & 13. & 12 & $=0.30$ \\
\hline 676 & 2 & $8: 58$ & $-47: 2$ & $\ldots$ & 15. & 14. & 13. & 12 & 12 & $=0.71$ \\
\hline 677 & 4 & 8:59:18.00 & $-47: 24: 5.53$ & \pm 0.03 & $=0.03$ & 13.5 & 13.28 & 13.17 & 12.0 & $\ldots$ \\
\hline 678 & 4 & $8: 59$ & $-47: 23: 36.97$ & & 15.5 & 14. & 13. & 13. & 11. & \\
\hline 679 & 2 & $8: 58:$ & $-47: 32$ & & 15.6 & 14.4 & 13.47 & 13. & 12 . & \\
\hline 680 & 4 & 8:59: & $-47: 3$ & 13.50 & 12.7 & 12.5 & 12.2 & 12 . & 10 & \\
\hline 681 & 2 & $8:$ & $-47: 29$ & $16.56 \pm 0.17$ & 14.7 & 13.6 & 13.1 & 12. & 12. & \\
\hline 682 & 2 & $8: 58$ & $-47: 25: 47.10$ & & 14.7 & 14. & 13. & 12. & 11 & \\
\hline 683 & 1 & $8: 57$ & $-47: 19: 31.05$ & 0.03 & 0.02 & 13.6 & 13. & 13. & 12 & \\
\hline 84 & 1 & 8 & $-47: 2$ & 16.2 & 14 & 14 . & 13 & 13 & 12 & \\
\hline 685 & 2 & $8:$ & $-47: 19$ & 0.05 & 14. & 13.6 & 13. & 13. & 12 . & $\ldots$ \\
\hline 686 & 2 & $8: 58: 15.28$ & $-47: 24: 6.54$ & 0.22 & & & & & & \\
\hline 687 & 2 & 8:59:5.40 & $-47: 19: 31.73$ & $15.80 \pm 0.08$ & 14.3 & 13. & 13. & 13 & 12 . & \\
\hline 688 & 2 & & $-47: 2$ & 0.06 & & & & & & . \\
\hline 689 & 2 & $8: 58$ & $-47: 21$ & $16.47 \pm 0.12$ & 15.01 & 14.3 & 13.69 & 13.34 & $12.99 \pm 0.17$ & 13.0 \\
\hline 690 & 2 & 8:58:44.67 & $-47: 20: 34.00$ & & $15.90 \pm 0.14$ & 14.86 & 14.12 & 13.60 & $12.75 \pm 0.17$ & \\
\hline 691 & 1 & 8:58:3.71 & $-47: 29: 52.98$ & & $15.34 \pm 0.10$ & 14.35 & 13.7 & 13 & 13. & \\
\hline 692 & 2 & $8: 58: 52.66$ & $-47: 23: 24.41$ & $14.09 \pm 0.03$ & $13.40 \pm 0.03$ & 13.12 & 13. & 13. & 12 . & $\cdots$ \\
\hline 694 & 4 & $8: 59:$ & $-47: 26: 41.34$ & & & 14.68 & 13.3 & 12.7 & 11 . & \\
\hline 695 & 4 & 8:58:24.42 & $-47: 31: 16.95$ & $15.74 \pm 0.08$ & $=0.05$ & $12.86 \pm 0.04$ & $12.26 \pm 0.07$ & 12.1 & 11.4 & \\
\hline 696 & 1 & 8:13.13 & $-47: 31: 23.74$ & $17.14 \pm 0.22$ & $14.55 \pm 0.04$ & $13.52 \pm 0.04$ & $12.91 \pm 0.09$ & 12.7 & 11. & \\
\hline 697 & 4 & & $-47: 30: 41.58$ & $12.38 \pm 0.03$ & $11.98 \pm 0.02$ & & 11. & & 11 & \\
\hline 698 & 2 & & $-47: 23: 5.36$ & $13.41 \pm 0.03$ & $12.64 \pm 0.03$ & $12.14 \pm 0.03$ & & & & \\
\hline 699 & 4 & 8:59:27.05 & $-47: 27: 14.62$ & $15.27 \pm 0.05$ & $13.67 \pm 0.03$ & $13.02 \pm 0.04$ & $12.57 \pm 0.08$ & $12.52 \pm 0.09$ & $11.65 \pm 0.19$ & \\
\hline 700 & 2 & $8: 58: 15.81$ & $-47: 24: 13.15$ & & $15.46 \pm 0.10$ & & 13. & 13.1 & 13.0 & 0.30 \\
\hline 701 & 1 & 8:57:55.15 & $-47: 23: 45.19$ & $16.09 \pm 0.09$ & $14.72 \pm 0.06$ & 0.07 & 13.92 & 13.7 & $13.2^{\prime}$ & $12.80 \pm 0.25$ \\
\hline 702 & 4 & & $-47: 23$ & $14.65 \pm 0.03$ & & 12.5 & & & 11. & \\
\hline 703 & 4 & 8:59:24.30 & $-47: 32: 41.46$ & $15.32 \pm 0.06$ & $12.79 \pm 0.03$ & $11.65 \pm 0.03$ & $10.83 \pm 0.04$ & $10.69 \pm 0.05$ & $9.94 \pm 0.20$ & \\
\hline 704 & 2 & 8:58:18.80 & $-47: 30: 59.35$ & $\ldots$ & $16.05 \pm 0.15$ & $14.30 \pm 0.08$ & $13.33 \pm 0.11$ & $13.16 \pm 0.12$ & $12.67 \pm 0.18$ & \\
\hline 705 & 1 & 8:58:6.99 & $-47: 31: 10.52$ & $\cdots$ & $16.25 \pm 0.19$ & $14.31 \pm 0.08$ & $13.35 \pm 0.11$ & $12.99 \pm 0.11$ & $12.73 \pm 0.13$ & $12.78 \pm 0.25$ \\
\hline
\end{tabular}


Table 11-Continued

\begin{tabular}{|c|c|c|c|c|c|c|c|c|c|}
\hline $\begin{array}{lc}S p . & \text { Ep. } \\
\text { ID } & \text { ID }\end{array}$ & $\begin{array}{c}\mathrm{RA} \\
\mathrm{J} 2000\end{array}$ & $\begin{array}{c}\text { Dec } \\
\text { J2000 }\end{array}$ & $\begin{array}{c}\mathrm{J} \\
{[\mathrm{mag} .]}\end{array}$ & $\begin{array}{c}\mathrm{H} \\
{[\mathrm{mag} .]}\end{array}$ & $\begin{array}{c}\mathrm{K} \\
{[\mathrm{mag} .]}\end{array}$ & $\begin{array}{l}3.6 \mu m \\
\text { [mag.] }\end{array}$ & $\begin{array}{l}4.5 \mu m \\
\text { [mag.] }\end{array}$ & $\begin{array}{l}5.8 \mu \mathrm{m} \\
\text { [mag.] }\end{array}$ & $\begin{array}{l}8.0 \mu \mathrm{m} \\
\text { [mag.] }\end{array}$ \\
\hline
\end{tabular}

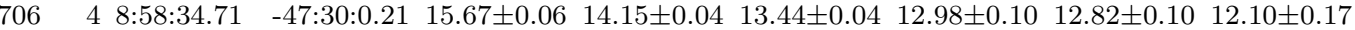

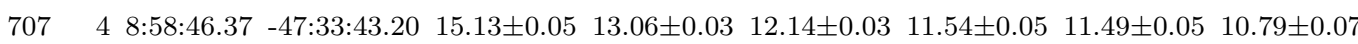

$\ldots$

$\begin{array}{llllllllll}708 & 1 & 9: 0: 8.19 & -47: 32: 34.14 & 14.79 \pm 0.04 & 13.73 \pm 0.03 & 13.21 \pm 0.04 & 12.85 \pm 0.09 & 12.72 \pm 0.10 & 12.15 \pm 0.18\end{array}$

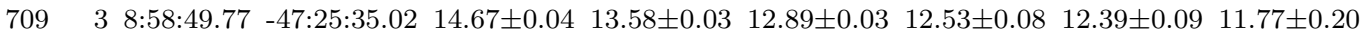

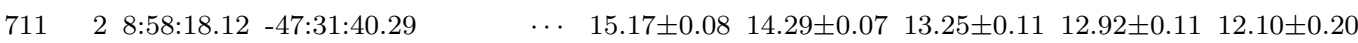

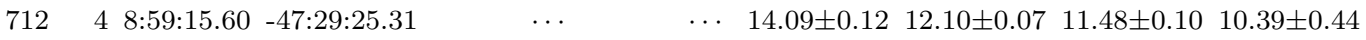

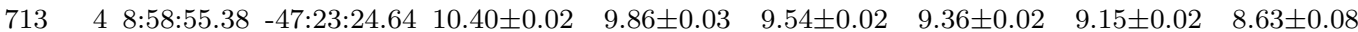

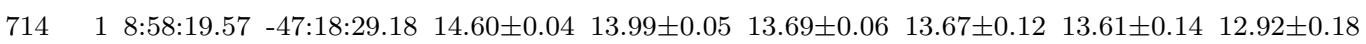

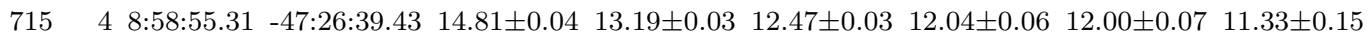

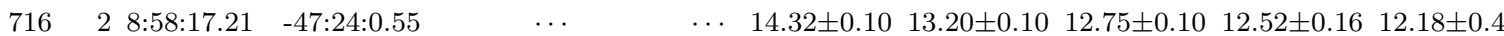

$\begin{array}{lllllllllll}717 & 2 & 8: 59: 4.82 & -47: 22: 36.47 & \ldots & \ldots & 14.70 \pm 0.10 & 12.79 \pm 0.08 & 12.29 \pm 0.08 & 12.03 \pm 0.19 & \ldots\end{array}$

$\begin{array}{llllllllllll}718 & 3 & 8: 58: 20.61 & -47: 31: 26.81 & \cdots & 15.68 \pm 0.12 & 14.61 \pm 0.09 & 13.86 \pm 0.14 & 13.66 \pm 0.15 & 12.43 \pm 0.18 & \cdots\end{array}$

$\begin{array}{lllllllllll}719 & 1 & 8: 58: 4.46 & -47: 22: 52.42 & 10.34 \pm 0.03 & 9.54 \pm 0.03 & 9.07 \pm 0.02 & 9.03 \pm 0.05 & 8.64 \pm 0.04 & 7.72 \pm 0.17 & \ldots\end{array}$

$\begin{array}{llllllllllll}720 & 4 & 8: 58: 30.34 & -47: 32: 15.63 & \ldots & \cdots & 14.31 \pm 0.07 & 12.51 \pm 0.07 & 12.04 \pm 0.07 & 11.86 \pm 0.19 & \ldots\end{array}$

$\begin{array}{lllllllllll}721 & 1 & 8: 58: 3.37 & -47: 25: 15.68 & 15.61 \pm 0.07 & 14.35 \pm 0.05 & 13.90 \pm 0.06 & 13.18 \pm 0.10 & 12.80 \pm 0.10 & 12.46 \pm 0.12 & 12.09 \pm 0.46\end{array}$

$\begin{array}{llllllllllll}722 & 1 & 8: 58: 4.80 & -47: 16: 19.31 & 15.35 \pm 0.06 & 14.60 \pm 0.05 & 14.36 \pm 0.08 & 14.17 \pm 0.16 & 14.17 \pm 0.19 & 13.41 \pm 0.19 & \ldots\end{array}$

$\begin{array}{lllllllllll}723 & 4 & 8: 59: 30.69 & -47: 23: 52.14 & 14.05 \pm 0.03 & 12.17 \pm 0.03 & 11.35 \pm 0.02 & 10.84 \pm 0.03 & 10.82 \pm 0.04 & 10.24 \pm 0.06 & \ldots\end{array}$

$\begin{array}{lllllllllll}724 & 2 & 9: 0: 0.30 & -47: 22: 11.17 & 15.66 \pm 0.09 & 14.14 \pm 0.05 & 13.52 \pm 0.05 & 12.92 \pm 0.09 & 12.70 \pm 0.10 & 11.92 \pm 0.13 & \ldots\end{array}$

$\begin{array}{lllllllllll}725 & 2 & 8: 59: 56.91 & -47: 19: 24.36 & 14.99 \pm 0.06 & 14.20 \pm 0.05 & 13.81 \pm 0.06 & 13.45 \pm 0.11 & 13.34 \pm 0.13 & 12.42 \pm 0.17 & \ldots\end{array}$

$\begin{array}{llllllllllll}726 & 1 & 9: 0: 11.27 & -47: 28: 4.77 & 13.70 \pm 0.03 & 12.47 \pm 0.03 & 11.99 \pm 0.03 & 11.62 \pm 0.05 & 11.61 \pm 0.06 & 11.08 \pm 0.06 & \ldots\end{array}$

$\begin{array}{llllllllllll}727 & 1 & 9: 0: 14.76 & -47: 24: 11.22 & 15.29 \pm 0.05 & 14.37 \pm 0.05 & 14.06 \pm 0.08 & 13.67 \pm 0.13 & 13.59 \pm 0.14 & 12.60 \pm 0.16 & \ldots\end{array}$

$\begin{array}{llllllllllll}728 & 1 & 8: 59: 4.84 & -47: 18: 20.54 & 14.71 \pm 0.04 & 13.27 \pm 0.03 & 12.59 \pm 0.03 & 12.27 \pm 0.07 & 12.23 \pm 0.08 & 11.58 \pm 0.13 & \ldots\end{array}$

$\begin{array}{lllllllllll}729 & 1 & 8: 59: 15.76 & -47: 17: 14.76 & \cdots & 15.70 \pm 0.14 & 14.47 \pm 0.09 & 13.80 \pm 0.13 & 13.40 \pm 0.13 & 13.06 \pm 0.15 & \ldots\end{array}$

$\begin{array}{lllllllllll}730 & 2 & 8: 59: 26.91 & -47: 20: 25.36 & 13.23 \pm 0.02 & 12.62 \pm 0.02 & 12.44 \pm 0.03 & 12.25 \pm 0.07 & 12.27 \pm 0.08 & 11.58 \pm 0.16 & \ldots\end{array}$

$\begin{array}{lllllllllll}731 & 1 & 9: 0: 10.03 & -47: 18: 21.31 & 16.02 \pm 0.09 & 13.73 \pm 0.03 & 12.64 \pm 0.03 & 11.82 \pm 0.06 & 11.87 \pm 0.07 & 10.99 \pm 0.09 & \ldots\end{array}$

$\begin{array}{llllllllllll}732 & 1 & 9: 0: 26.47 & -47: 26: 27.64 & 16.05 \pm 0.08 & 14.52 \pm 0.05 & 13.93 \pm 0.05 & 13.50 \pm 0.12 & 13.42 \pm 0.13 & 12.57 \pm 0.19 & \ldots\end{array}$

$\begin{array}{lllllllllll}733 & 2 & 8: 59: 19.02 & -47: 22: 18.25 & 16.62 \pm 0.14 & 13.59 \pm 0.03 & 12.19 \pm 0.03 & 11.17 \pm 0.04 & 10.98 \pm 0.04 & 10.40 \pm 0.08 & \cdots\end{array}$

$\begin{array}{lllllllllll}734 & 1 & 8: 59: 35.46 & -47: 16: 17.62 & 16.39 \pm 0.14 & 15.36 \pm 0.12 & 14.91 \pm 0.15 & 14.48 \pm 0.18 & 14.10 \pm 0.18 & 13.30 \pm 0.20 & \ldots\end{array}$

$\begin{array}{lllllllllll}735 & 4 & 8: 59: 35.23 & -47: 25: 22.69 & 14.41 \pm 0.03 & 13.73 \pm 0.04 & 13.48 \pm 0.05 & 13.16 \pm 0.10 & 13.14 \pm 0.12 & 12.48 \pm 0.15 & \ldots\end{array}$

$\begin{array}{llllllllllll}736 & 1 & 8: 59: 55.07 & -47: 30: 33.31 & 15.32 \pm 0.06 & 14.16 \pm 0.04 & 13.63 \pm 0.05 & 13.05 \pm 0.11 & 13.03 \pm 0.13 & 11.53 \pm 0.20 & \ldots\end{array}$

$\begin{array}{lllllllllll}737 & 4 & 8: 59: 44.53 & -47: 25: 23.90 & \ldots & 16.09 \pm 0.20 & 14.94 \pm 0.13 & 13.77 \pm 0.14 & 13.16 \pm 0.12 & 12.12 \pm 0.18 & \ldots\end{array}$

$\begin{array}{llllllllllll}738 & 2 & 9: 0: 1.14 & -47: 21: 6.24 & 14.79 \pm 0.04 & 14.17 \pm 0.04 & 13.94 \pm 0.06 & 13.60 \pm 0.12 & 13.61 \pm 0.14 & 12.85 \pm 0.18 & \ldots\end{array}$

$\begin{array}{llllllllllll}739 & 1 & 9: 0: 23.53 & -47: 25: 40.13 & 15.71 \pm 0.08 & 14.36 \pm 0.06 & 13.78 \pm 0.06 & 13.33 \pm 0.11 & 13.33 \pm 0.13 & 12.61 \pm 0.15 & \ldots\end{array}$

$\begin{array}{lllllllllll}740 & 2 & 8: 59: 32.78 & -47: 18: 46.69 & 15.75 \pm 0.08 & 14.75 \pm 0.06 & 14.34 \pm 0.08 & 14.10 \pm 0.15 & 13.98 \pm 0.17 & 13.27 \pm 0.18 & \ldots\end{array}$

$\begin{array}{llllllllllll}741 & 1 & 9: 0: 4.63 & -47: 27: 50.81 & 15.24 \pm 0.07 & 14.04 \pm 0.04 & 13.46 \pm 0.04 & 13.08 \pm 0.10 & 13.07 \pm 0.11 & 12.15 \pm 0.13 & \ldots\end{array}$

$\begin{array}{llllllllllll}742 & 3 & 8: 59: 49.89 & -47: 28: 8.35 & 13.00 \pm 0.03 & 12.60 \pm 0.03 & 12.30 \pm 0.03 & 12.07 \pm 0.07 & 11.94 \pm 0.07 & 10.41 \pm 0.08 & \ldots\end{array}$

$\begin{array}{lllllllllll}743 & 1 & 8: 59: 52.77 & -47: 31: 25.31 & 14.06 \pm 0.03 & 13.53 \pm 0.03 & 13.45 \pm 0.04 & 13.35 \pm 0.12 & 13.45 \pm 0.14 & 12.19 \pm 0.18 & \ldots\end{array}$

$\begin{array}{lllllllllll}744 & 2 & 8: 59: 44.22 & -47: 20: 57.65 & 14.39 \pm 0.03 & 13.11 \pm 0.03 & 12.57 \pm 0.03 & 12.33 \pm 0.07 & 12.33 \pm 0.08 & 11.69 \pm 0.15 & \ldots\end{array}$

$\begin{array}{llllllllllll}745 & 1 & 9: 0: 11.85 & -47: 27: 44.52 & 16.24 \pm 0.13 & 14.77 \pm 0.08 & 14.08 \pm 0.07 & 13.44 \pm 0.13 & 13.10 \pm 0.12 & 11.79 \pm 0.16 & \ldots\end{array}$

$\begin{array}{lllllllllll}746 & 1 & 8: 59: 59.36 & -47: 28: 28.39 & 16.63 \pm 0.14 & 15.10 \pm 0.08 & 14.34 \pm 0.08 & 13.70 \pm 0.13 & 13.36 \pm 0.13 & 12.37 \pm 0.17 & \ldots\end{array}$

$\begin{array}{lllllllllll}747 & 2 & 8: 59: 37.95 & -47: 19: 50.73 & 14.31 \pm 0.03 & 13.70 \pm 0.03 & 13.38 \pm 0.03 & 13.25 \pm 0.10 & 13.20 \pm 0.12 & 12.52 \pm 0.16 & \ldots\end{array}$

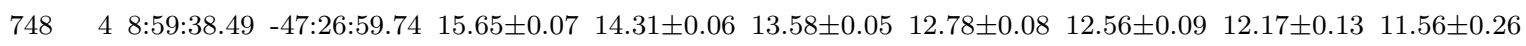

$\begin{array}{llllllllllll}749 & 2 & 9: 0: 3.46 & -47: 20: 6.97 & 15.28 \pm 0.06 & 14.02 \pm 0.05 & 13.44 \pm 0.05 & 13.10 \pm 0.10 & 13.05 \pm 0.11 & 12.47 \pm 0.19 & \ldots\end{array}$

$\begin{array}{llllllllllll}750 & 1 & 9: 0: 14.60 & -47: 21: 39.77 & 14.68 \pm 0.04 & 14.02 \pm 0.04 & 13.86 \pm 0.05 & 13.65 \pm 0.12 & 13.58 \pm 0.14 & 12.99 \pm 0.17 & \ldots\end{array}$

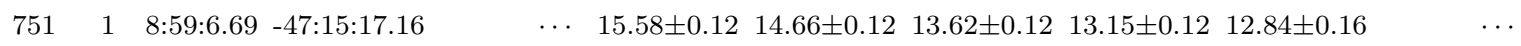


Table 11-Continued

\begin{tabular}{|c|c|c|c|c|c|c|c|c|c|c|}
\hline $\begin{array}{l}S p . \\
\text { ID }\end{array}$ & $\begin{array}{l}\text { Ep. } \\
\text { ID }\end{array}$ & $\begin{array}{c}\text { RA } \\
\text { J2000 }\end{array}$ & $\begin{array}{c}\text { Dec } \\
\text { J2000 }\end{array}$ & $\begin{array}{c}\mathrm{J} \\
\text { [mag.] }\end{array}$ & $\begin{array}{c}\mathrm{H} \\
\text { [mag.] }\end{array}$ & $\begin{array}{c}\mathrm{K} \\
{[\mathrm{mag} .]}\end{array}$ & $\begin{array}{l}3.6 \mu \mathrm{m} \\
\text { [mag.] }\end{array}$ & $\begin{array}{l}4.5 \mu m \\
\text { [mag.] }\end{array}$ & $\begin{array}{l}5.8 \mu \mathrm{m} \\
\text { [mag.] }\end{array}$ & $\begin{array}{l}8.0 \mu m \\
\text { [mag.] }\end{array}$ \\
\hline 752 & 1 & $59: 1.98$ & $-47: 14: 51.33$ & $14.27 \pm 0.03$ & $13.52 \pm 0.03$ & $13.23 \pm 0.03$ & $13.03 \pm 0.10$ & $12.99 \pm 0.11$ & $12.30 \pm 0.19$ & \\
\hline 753 & 1 & $9: 0: 10.46$ & $-47: 29: 53.13$ & $15.22 \pm 0.06$ & $14.17 \pm 0.04$ & $13.63 \pm 0.05$ & $13.20 \pm 0.11$ & $13.09 \pm 0.12$ & $12.11 \pm 0.15$ & 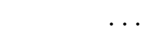 \\
\hline 754 & 1 & $9: 0: 14.39$ & $-47: 23: 45.79$ & $14.96 \pm 0.04$ & $13.74 \pm 0.04$ & $13.25 \pm 0.04$ & $12.98 \pm 0.09$ & $12.97 \pm 0.11$ & $12.38 \pm 0.13$ & \\
\hline 755 & 2 & $8: 59: 40.77$ & $-47: 21: 5.13$ & $14.89 \pm 0.05$ & $13.41 \pm 0.03$ & $12.80 \pm 0.03$ & $12.43 \pm 0.07$ & $12.41 \pm 0.08$ & $11.89 \pm 0.11$ & \\
\hline 758 & 1 & $8: 58: 17.67$ & $-47: 24: 29.88$ & $\cdots$ & $\cdots$ & . & $13.28 \pm 0.10$ & $13.04 \pm 0.11$ & $12.68 \pm 0.15$ & \\
\hline 759 & 2 & $8: 58: 21.70$ & $-47: 22: 56.12$ & $\cdots$ & $\ldots$ & $\cdots$ & $13.70 \pm 0.13$ & $13.47 \pm 0.14$ & $12.75 \pm 0.18$ & . \\
\hline 760 & 4 & $8: 58: 18.76$ & $-47: 24: 22.30$ & $\cdots$ & $\cdots$ & $\cdots$ & $13.94 \pm 0.14$ & $13.25 \pm 0.12$ & $13.12 \pm 0.22$ & $12.65 \pm 0.49$ \\
\hline 761 & 1 & $8: 58: 21.62$ & $-47: 23: 48.73$ & $\ldots$ & $\cdots$ & $\ldots$ & $13.60 \pm 0.12$ & $13.19 \pm 0.13$ & $12.83 \pm 0.17$ & $11.95 \pm 0.26$ \\
\hline 763 & 2 & $8: 58: 15.54$ & $-47: 26: 57.93$ & $\ldots$ & $\ldots$ & $\ldots$ & $14.05 \pm 0.15$ & $13.43 \pm 0.13$ & $12.77 \pm 0.18$ & $11.80 \pm 0.27$ \\
\hline 764 & 2 & $8: 58: 26.74$ & $-47: 23: 23.43$ & $\cdots$ & $\cdots$ & $\cdots$ & $13.80 \pm 0.14$ & $13.15 \pm 0.12$ & $12.29 \pm 0.15$ & $\cdots$ \\
\hline 765 & 1 & $8: 58: 10.94$ & $-47: 30: 37.90$ & $\ldots$ & $\ldots$ & $\ldots$ & $14.44 \pm 0.19$ & $14.13 \pm 0.19$ & $12.84 \pm 0.19$ & $\ldots$ \\
\hline 766 & 2 & $8: 58: 20.55$ & $-47: 30: 49.23$ & $\cdots$ & $\cdots$ & .. & $13.58 \pm 0.12$ & $12.87 \pm 0.10$ & $12.59 \pm 0.22$ & $12.15 \pm 0.66$ \\
\hline 767 & 4 & $8: 58: 26.17$ & $-47: 30: 41.48$ & $\ldots$ & $\ldots$ & . & $14.12 \pm 0.17$ & $12.30 \pm 0.08$ & $11.33 \pm 0.11$ & $10.86 \pm 0.38$ \\
\hline 768 & 2 & $8: 58: 28.20$ & $-47: 37: 20.01$ & $\ldots$ & $\ldots$ & .. & $14.35 \pm 0.17$ & $13.80 \pm 0.16$ & $13.45 \pm 0.20$ & $12.40 \pm 0.22$ \\
\hline 769 & 1 & $8: 59: 16.48$ & $-47: 16: 28.73$ & $\cdots$ & $\ldots$ & $\ldots$ & $13.89 \pm 0.14$ & $13.39 \pm 0.13$ & $13.18 \pm 0.18$ & $12.79 \pm 0.25$ \\
\hline 770 & 2 & $8: 58: 32.91$ & $-47: 38: 50.48$ & $\cdots$ & $\cdots$ & $\cdots$ & $14.30 \pm 0.17$ & $13.82 \pm 0.16$ & $12.27 \pm 0.16$ & $11.01 \pm 0.26$ \\
\hline 771 & 3 & 8:58:40.36 & $-47: 36: 4.87$ & $\cdots$ & $\cdots$ & .. & $14.34 \pm 0.18$ & $14.02 \pm 0.18$ & $12.74 \pm 0.19$ & $\cdots$ \\
\hline 772 & 3 & $8: 58: 41.31$ & $-47: 36: 0.94$ & $\ldots$ & $\ldots$ & .. & $14.02 \pm 0.15$ & $13.53 \pm 0.19$ & $12.78 \pm 0.19$ & $\ldots$ \\
\hline 773 & 2 & $8: 59: 16.10$ & $-47: 22: 58.05$ & $\cdots$ & $\ldots$ & $\ldots$ & $12.78 \pm 0.09$ & $12.57 \pm 0.09$ & $11.66 \pm 0.12$ & $\ldots$ \\
\hline 775 & 4 & $8: 59: 15.31$ & $-47: 27: 19.50$ & $\ldots$ & $\ldots$ & $\ldots$ & $14.47 \pm 0.19$ & $13.30 \pm 0.13$ & 12.10 & $\ldots$ \\
\hline 776 & 4 & $8: 58: 54.64$ & $-47: 38: 2.19$ & $\cdots$ & $\cdots$ & .. & $13.85 \pm 0.14$ & 13.38 & 12.63 & $\ldots$ \\
\hline 777 & 2 & $8: 58: 59.49$ & $-47: 38: 19.84$ & $\ldots$ & $\ldots$ & .. & $13.92 \pm 0.14$ & $13.73 \pm 0.15$ & $13.01 \pm 0.19$ & $\ldots$ \\
\hline 778 & 1 & $8: 59: 46.84$ & $-47: 17: 43.17$ & $\cdots$ & $\cdots$ & $\cdots$ & $14.36 \pm 0.18$ & $13.54 \pm 0.15$ & $13.19 \pm 0.17$ & $\cdots$ \\
\hline 780 & 4 & $8: 59: 4$ & $-47: 31: 23.10$ & $\ldots$ & $\ldots$ & $\ldots$ & $13.24 \pm 0.11$ & $12.55 \pm 0.11$ & $10.75 \pm 0.09$ & . \\
\hline 781 & 2 & $8: 58: 15.30$ & $-47: 22: 17.38$ & $\cdots$ & $\cdots$ & . & $14.31 \pm 0.17$ & $13.73 \pm 0.15$ & $13.08 \pm 0.19$ & $11.78 \pm 0.20$ \\
\hline 782 & 3 & $8: 58: 23.66$ & $-47: 32: 11.95$ & $\ldots$ & $\cdots$ & & $14.25 \pm 0.16$ & $13.87 \pm 0.17$ & $13.00 \pm 0.20$ & $\cdots$ \\
\hline 783 & 4 & $8: 58: 47.58$ & $-47: 29: 26.61$ & $\ldots$ & $\ldots$ & & $13.23 \pm 0.13$ & $12.50 \pm 0.10$ & $11.10 \pm 0.18$ & $\ldots$ \\
\hline 784 & 3 & $8: 58: 48.32$ & $-47: 31: 1.03$ & $\cdots$ & $\cdots$ & $\cdots$ & $13.26 \pm 0.12$ & $12.72 \pm 0.11$ & $11.41 \pm 0.20$ & $\cdots$ \\
\hline 785 & 4 & $8: 58: 39.32$ & $-47: 34: 55.32$ & $\ldots$ & $\ldots$ & $\ldots$ & $13.82 \pm 0.16$ & $13.16 \pm 0.14$ & $12.48 \pm 0.15$ & $\ldots$ \\
\hline 786 & 2 & $9: 0: 13.42$ & $-47: 37: 27.09$ & $15.55 \pm 0.05$ & $14.81 \pm 0.07$ & \pm 0.08 & $13.99 \pm 0.14$ & $13.98 \pm 0.17$ & $13.32 \pm 0.20$ & \\
\hline 787 & 2 & $9: 0: 36.59$ & $-47: 42: 5.82$ & $16.77 \pm 0.18$ & $15.52 \pm 0.13$ & $14.64 \pm 0.12$ & $14.22 \pm 0.16$ & $14.05 \pm 0.18$ & $13.53 \pm 0.19$ & $12.95 \pm 0.22$ \\
\hline 788 & 2 & 9:0:41.01 & $-47: 40: 8.41$ & $\cdots$ & $\cdots$ & $15.04 \pm 0.14$ & $13.84 \pm 0.14$ & $13.52 \pm 0.14$ & $13.18 \pm 0.17$ & $\cdots$ \\
\hline 789 & 2 & $8: 58: 36.19$ & $-47: 41: 13.37$ & $16.87 \pm 0.16$ & $15.41 \pm 0.09$ & $14.94 \pm 0.12$ & $14.23 \pm 0.16$ & $13.90 \pm 0.16$ & $13.49 \pm 0.18$ & $12.92 \pm 0.23$ \\
\hline 790 & 4 & $8: 58: 47.47$ & $-47: 34: 43.87$ & & $15.45 \pm 0.11$ & $14.01 \pm 0.07$ & $12.91 \pm 0.10$ & $12.71 \pm 0.11$ & $12.02 \pm 0.18$ & \\
\hline 791 & 4 & $8: 58: 37.31$ & $-47: 34: 9.69$ & $17.07 \pm 0.19$ & $14.53 \pm 0.03$ & $13.33 \pm 0.03$ & $12.58 \pm 0.08$ & $12.48 \pm 0.09$ & $12.02 \pm 0.13$ & $11.55 \pm 0.35$ \\
\hline 792 & 2 & $8: 58: 34.92$ & $-47: 43: 3.83$ & $\ldots$ & $15.61 \pm 0.16$ & $14.94 \pm 0.13$ & $13.78 \pm 0.13$ & $13.52 \pm 0.14$ & $13.05 \pm 0.16$ & $12.51 \pm 0.20$ \\
\hline 793 & 2 & 8:58:41.69 & $-47: 42: 36.40$ & $16.06 \pm 0.09$ & $14.78 \pm 0.06$ & $14.27 \pm 0.07$ & $13.87 \pm 0.14$ & $13.66 \pm 0.15$ & $13.20 \pm 0.18$ & $\cdots$ \\
\hline 794 & 4 & $8: 58: 34.78$ & $-47: 34: 5.65$ & $\ldots$ & $15.85 \pm 0.12$ & $14.43 \pm 0.08$ & $13.32 \pm 0.11$ & $13.07 \pm 0.12$ & $12.31 \pm 0.13$ & $\ldots$ \\
\hline 795 & 2 & $9: 0: 9.07$ & $-47: 41: 41.67$ & $\cdots$ & $15.71 \pm 0.12$ & $14.84 \pm 0.12$ & $13.85 \pm 0.14$ & $13.26 \pm 0.12$ & $12.93 \pm 0.16$ & $12.54 \pm 0.32$ \\
\hline 799 & 2 & $8: 58: 39.86$ & $-47: 46: 10.51$ & $\ldots$ & $\ldots$ & $\ldots$ & $13.95 \pm 0.15$ & $14.04 \pm 0.18$ & $13.28 \pm 0.18$ & $13.54 \pm 0.27$ \\
\hline 800 & 2 & $8: 58: 50.44$ & $-47: 42: 28.84$ & $\cdots$ & $\cdots$ & $\cdots$ & $14.37 \pm 0.17$ & $13.77 \pm 0.16$ & $13.38 \pm 0.18$ & $12.05 \pm 0.17$ \\
\hline 801 & 2 & 9:0:10.01 & $-47: 36: 10.54$ & $\cdots$ & $\cdots$ & $\ldots$ & $13.72 \pm 0.13$ & $13.11 \pm 0.12$ & $12.88 \pm 0.18$ & $12.22 \pm 0.50$ \\
\hline 802 & 2 & $8: 58: 20.70$ & $-47: 33: 37.89$ & $\cdots$ & $\ldots$ & $\ldots$ & $14.20 \pm 0.16$ & $13.87 \pm 0.16$ & $12.92 \pm 0.19$ & \\
\hline
\end{tabular}


Table 12. Astrometry \& Photometry of Candidate Variable YSOs in RCW 38.

\begin{tabular}{|c|c|c|c|c|c|c|c|c|c|c|c|c|}
\hline $\begin{array}{l}S p . \\
\text { ID }\end{array}$ & $\begin{array}{l}\text { Ep. } \\
\text { ID }\end{array}$ & $\begin{array}{c}\text { RA } \\
\text { J2000 }\end{array}$ & $\begin{array}{c}\text { Dec } \\
\text { J2000 }\end{array}$ & $\begin{array}{c}\mathrm{J} \\
\text { [mag.] }\end{array}$ & $\begin{array}{c}\mathrm{H} \\
\text { [mag.] }\end{array}$ & $\begin{array}{c}\mathrm{K} \\
\text { [mag.] }\end{array}$ & $\begin{array}{l}3.6 \mu \mathrm{m} \\
\text { [mag.] }\end{array}$ & $\begin{array}{l}4.5 \mu m \\
\text { [mag.] }\end{array}$ & $\begin{array}{l}5.8 \mu \mathrm{m} \\
\text { [mag.] }\end{array}$ & $\begin{array}{l}8.0 \mu \mathrm{m} \\
\text { [mag.] }\end{array}$ & $\begin{array}{c}A_{K} \\
{[\mathrm{mag} .]}\end{array}$ & Class \\
\hline oc1-v1 & 2 & $8: 58: 28.56$ & $-47: 39: 00.9$ & $9.991 \pm 0.026$ & $8.205 \pm 0.044$ & $7.413 \pm 0.026$ & & & $6.855 \pm 0.008$ & $6.813 \pm 0.012$ & 1.25 & III \\
\hline oc2-v2 & 3 & $8: 58: 59.40$ & $-47: 34: 16.6$ & $8.654 \pm 0.021$ & $7.784 \pm 0.039$ & $7.489 \pm 0.029$ & $7.385 \pm 0.008$ & $7.471 \pm 0.009$ & $7.365 \pm 0.063$ & $7.234 \pm 0.257$ & 0.28 & III \\
\hline v3 & 2 & 8:58:53.32 & $-47: 41: 02.9$ & $11.533 \pm 0.023$ & $10.446 \pm 0.027$ & $9.989 \pm 0.022$ & $9.784 \pm 0.021$ & $9.793 \pm 0.025$ & $9.747 \pm 0.0319$ & $9.660 \pm 0.045$ & 0.48 & III \\
\hline $\mathrm{v} 4$ & 2 & $8: 59: 44.28$ & $-47: 42: 44.9$ & $11.723 \pm 0.021$ & $10.379 \pm 0.025$ & $9.903 \pm 0.024$ & $9.603 \pm 0.019$ & $9.664 \pm 0.023$ & $9.533 \pm 0.029$ & $9.553 \pm 0.048$ & 0.78 & III \\
\hline v5 & 1 & $8: 59: 55.54$ & $-47: 40: 48.4$ & $11.615 \pm 0.021$ & $10.267 \pm 0.024$ & $9.763 \pm 0.024$ & $9.516 \pm 0.018$ & $9.326 \pm 0.020$ & $9.215 \pm 0.024$ & $9.192 \pm 0.038$ & 0.79 & III \\
\hline oc5-v6 & 1 & $8: 58: 20.07$ & $-47: 23: 48.6$ & $9.094 \pm 0.030$ & $8.372 \pm 0.049$ & $8.012 \pm 0.038$ & $\cdots$ & $8.110 \pm 0.012$ & $7.451 \pm 0.011$ & $7.249 \pm 0.018$ & $\cdots$ & III \\
\hline oc6-v7 & 1 & $8: 57: 46.59$ & $-47: 32: 32.4$ & $9.793 \pm 0.021$ & $8.425 \pm 0.039$ & $7.710 \pm 0.036$ & .. & & $6.802 \pm 0.008$ & $6.723 \pm 0.012$ & 0.68 & III \\
\hline v8 & 1 & $8: 59: 59.74$ & $-47: 37: 51.4$ & $9.094 \pm 0.0223$ & $8.991 \pm 0.025$ & $8.951 \pm 0.023$ & $9.211 \pm 0.016$ & $8.997 \pm 0.017$ & $8.874 \pm 0.021$ & $8.973 \pm 0.034$ & $\cdots$ & III \\
\hline v9 & 1 & $8: 57: 54.23$ & $-47: 25: 54.7$ & & $12.765 \pm 0.070$ & & $11.714 \pm 0.051$ & $11.740 \pm 0.061$ & $11.413 \pm 0.069$ & $10.581 \pm 0.070$ & $\ldots$ & II \\
\hline oc7-v10 & 2 & $8: 58: 15.87$ & $-47: 30: 18.8$ & $11.92 \pm 0.024$ & $9.854 \pm 0.021$ & $8.975 \pm 0.021$ & $8.859 \pm 0.014$ & $8.537 \pm 0.014$ & $8.314 \pm 0.017$ & $8.265 \pm 0.025$ & 1.54 & III \\
\hline oc13-v12 & 3 & $8: 59: 27.44$ & $-47: 25: 36.4$ & $9.682 \pm 0.023$ & $8.037 \pm 0.027$ & $7.353 \pm 0.026$ & $7.143 \pm 0.006$ & $7.086 \pm 0.007$ & $6.921 \pm 0.008$ & $6.844 \pm 0.014$ & 1.10 & III \\
\hline oc15-v13 & 3 & 8:59:31.83 & $-47: 33: 21.5$ & $8.122 \pm 0.021$ & $7.368 \pm 0.034$ & $7.094 \pm 0.017$ & $7.118 \pm 0.007$ & $7.104 \pm 0.007$ & $6.998 \pm 0.010$ & $6.942 \pm 0.042$ & 0.12 & III \\
\hline v14 & 1 & $8: 59: 40.45$ & $-47: 33: 48.6$ & $10.879 \pm 0.023$ & $10.089 \pm 0.024$ & $9.860 \pm 0.024$ & $9.742 \pm 0.020$ & $9.803 \pm 0.025$ & $9.684 \pm 0.032$ & $9.656 \pm 0.071$ & 0.20 & III \\
\hline v15 & 2 & $8: 58: 29.41$ & $-47: 30: 06.4$ & $10.285 \pm 0.023$ & $10.019 \pm 0.021$ & $9.989 \pm 0.019$ & $10.052 \pm 0.024$ & $10.044 \pm 0.027$ & $9.966 \pm 0.049$ & $9.610 \pm 0.147 \mathrm{v}$ & $v \quad \cdots$ & III \\
\hline v16 & 2 & $8: 59: 19.50$ & $-47: 26: 01.3$ & $16.469 \pm 0.123$ & $14.456 \pm 0.057$ & $13.415 \pm 0.055$ & $12.627 \pm 0.079$ & $12.253 \pm 0.078$ & $11.854 \pm 0.174$ & $10.820 \pm 0.373$ & 1.06 & III \\
\hline v17 & 1 & 8:59:37.48 & $-47: 32: 38.8$ & $9.906 \pm 0.021$ & $9.131 \pm 0.024$ & $8.887 \pm 0.023$ & $8.992 \pm 0.015$ & $8.817 \pm 0.016$ & $8.752 \pm 0.023$ & $8.783 \pm 0.077$ & 0.18 & III \\
\hline v18 & 1 & $8: 58: 33.66$ & $-47: 20: 30.9$ & $13.346 \pm 0.027$ & $12.188 \pm 0.023$ & $11.755 \pm 0.024$ & $11.479 \pm 0.045$ & $11.454 \pm 0.053$ & $11.001 \pm 0.059$ & $11.152 \pm 0.140$ & 0.59 & III \\
\hline v19 & 3 & $8: 59: 15.60$ & $-47: 29: 25.3$ & $\ldots$ & .. & $14.090 \pm 0.116$ & $11.748 \pm 0.061$ & $10.986 \pm 0.056$ & $9.845 \pm 0.169$ & . & $\ldots$ & II \\
\hline v20 & 1 & 9: $0: 15.61$ & $-47: 22: 43.7$ & $11.627 \pm 0.022$ & $10.575 \pm 0.025$ & $10.175 \pm 0.023$ & $9.946 \pm 0.022$ & $9.933 \pm 0.026$ & $9.938 \pm 0.036$ & $9.906 \pm 0.071$ & 0.47 & III \\
\hline v21 & 1 & $8: 59: 27.91$ & $-47: 23: 34.6$ & $15.232 \pm 0.041$ & $13.697 \pm 0.041$ & $13.105 \pm 0.031$ & $12.697 \pm 0.082$ & $12.639 \pm 0.095$ & $12.379 \pm 0.327$ & $11.694 \pm 0.909$ & 0.98 & II \\
\hline oc23-v22 & 1 & $8: 59: 45.44$ & $-47: 18: 24.5$ & $7.135 \pm 0.023$ & $6.661 \pm 0.022$ & $6.567 \pm 0.021$ & & & $6.699 \pm 0.008$ & $6.576 \pm 0.011$ & $\ldots$ & III \\
\hline oc26-v23 & 1 & $8: 59: 49.06$ & $-47: 20: 11.1$ & $10.065 \pm 0.021$ & $8.791 \pm 0.032$ & $8.310 \pm 0.025$ & $8.574 \pm 0.0121$ & $8.215 \pm 0.012$ & $7.968 \pm 0.014$ & $7.928 \pm 0.019$ & 0.71 & III \\
\hline v24 & 1 & 8:59:58.96 & $-47: 19: 21.9$ & $11.527 \pm 0.021$ & $10.477 \pm 0.022$ & $10.000 \pm 0.023$ & $9.632 \pm 0.019$ & $9.352 \pm 0.020$ & $9.275 \pm 0.027$ & $9.219 \pm 0.059$ & 0.40 & III \\
\hline v25 & 4 & $8: 58: 24.23$ & $-47: 37: 13.1$ & $9.119 \pm 0.030$ & $7.462 \pm 0.053$ & $6.598 \pm 0.027$ & $6.591 \pm 0.006$ & $6.391 \pm 0.006$ & $6.018 \pm 0.006$ & $5.881 \pm 0.008$ & 1.01 & III \\
\hline
\end{tabular}


Table 13. Astrometry Photometry of candidate O-type stars in RCW 38.

\begin{tabular}{|c|c|c|c|c|c|c|c|c|c|c|c|}
\hline $\begin{array}{l}S p . \\
\text { ID }\end{array}$ & $\begin{array}{l}\text { Ep. } \\
\text { ID }\end{array}$ & $\begin{array}{c}\text { RA } \\
\text { J2000 }\end{array}$ & $\begin{array}{c}\text { Dec } \\
\text { J2000 }\end{array}$ & $\begin{array}{c}\mathrm{J} \\
{[\mathrm{mag} .]}\end{array}$ & $\begin{array}{c}\mathrm{H} \\
{[\mathrm{mag} .]}\end{array}$ & $\begin{array}{c}\mathrm{K} \\
{[\mathrm{mag} .]}\end{array}$ & $\begin{array}{l}3.6 \mu \mathrm{m} \\
\text { [mag.] }\end{array}$ & $\begin{array}{l}4.5 \mu m \\
\text { [mag.] }\end{array}$ & $\begin{array}{l}5.8 \mu \mathrm{m} \\
\text { [mag.] }\end{array}$ & $\begin{array}{l}8.0 \mu m \\
{[\mathrm{mag} .]}\end{array}$ & $\begin{array}{c}A_{K} \\
\text { [mag.] }\end{array}$ \\
\hline & 2 & & & & & & & & & & \\
\hline oc3 & 2 & & & & $10.334 \pm$ & & & & & & 45 \\
\hline 604 & 2 & & $-47: 3$ & & - & 21 & 0.013 & & & & \\
\hline oc5-v6 & 1 & & $-47: 23:$ & & $372 \pm$ & $8.012 \pm 0.038$ & & .012 & & & \\
\hline oc8 & 1 & & $-47:$ & & 9.321 & & & & & 8.10 & 1.16 \\
\hline 158 & 2 & & & & 11.1 & & .016 & & & & 2.73 \\
\hline oc10 & 4 & & & & & & 06 & & & & 0.54 \\
\hline & 1 & & & & & & .014 & & & & 41 \\
\hline c12 & 1 & & -47 & & & & $\ldots$ & & & & .10 \\
\hline $3-\mathrm{v} 12$ & 3 & & $-47:$ & & 7 & & .006 & & & & 1.10 \\
\hline & 4 & & $-47: 25: 08.8$ & .024 & & 23 & .012 & 8.6 & & & 1.10 \\
\hline & 1 & & & & & 9.001 & & 8.371 & & & 23 \\
\hline $0<20$ & 1 & & & & & & $\because \theta$ & & & & 0.28 \\
\hline 806 & 1 & & $-47: 2$ & 11.0 & 22 & 8.713 & $8.741 \pm 0.013$ & 14 & 16 & & 1.05 \\
\hline oc22 & 1 & & $-47: 16: 54.3$ & & $7.771 \pm 0.026$ & $7.479 \pm 0.020$ & & $7.723 \pm 0.010$ & & & \\
\hline oc23-v22 & 1 & & $-47: 18: 24.5$ & & 0.023 & 6.567 & & & & & \\
\hline & 1 & & $-47: 2$ & & & & $8.700 \pm 0.01$ & & & & 0.80 \\
\hline oc25 & 1 & .31 & $-47: 20: 38.7$ & $=0.021$ & $8.979 \pm 0.025$ & $8.305 \pm 0.032$ & & $8.229 \pm 0.012$ & 14 & 037 & 0.89 \\
\hline oc26-v23 & 1 & 9.06 & $-47: 20: 11.1$ & $10.06 \pm 0.021$ & $8.791 \pm 0.032$ & $8.310 \pm 0.024$ & 0.012 & $8.214 \pm 0.012$ & 7.96 & 7.92 & 0.71 \\
\hline 808 & 1 & 9:0 & $-47: 21: 09.3$ & 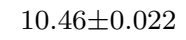 & 4 & & 0.013 & $8.38^{\prime}$ & & 8.16 & 0.80 \\
\hline & 1 & & & & & & & & & & 0.99 \\
\hline 811 & 4 & 1.23 & $-47: 37: 13.1$ & $120 \pm 0.030$ & .050 & $6.600 \pm 0.030$ & $6.590 \pm 0.010$ & $6.390=$ & $6.020 \pm 0.010$ & 5.880 & 1.01 \\
\hline
\end{tabular}

Table 14. Astrometry \& Photometry of stars associated with $24 \mu \mathrm{m}$ emission in RCW 38.

\begin{tabular}{|c|c|c|c|c|c|c|c|c|c|c|c|}
\hline $\begin{array}{l}S p . \\
\text { ID }\end{array}$ & $\begin{array}{l}\text { Ep. } \\
\text { ID }\end{array}$ & $\begin{array}{c}\text { RA } \\
\text { J2000 }\end{array}$ & $\begin{array}{c}\text { Dec } \\
\text { J2000 }\end{array}$ & $\begin{array}{c}\mathrm{J} \\
\text { [mag.] }\end{array}$ & $\begin{array}{c}\mathrm{H} \\
\text { [mag.] }\end{array}$ & $\begin{array}{c}\mathrm{K} \\
{[\mathrm{mag} .]}\end{array}$ & $\begin{array}{l}3.6 \mu \mathrm{m} \\
{[\mathrm{mag} .]}\end{array}$ & $\begin{array}{l}4.5 \mu m \\
\text { [mag.] }\end{array}$ & $\begin{array}{l}5.8 \mu m \\
\text { [mag.] }\end{array}$ & $\begin{array}{l}8.0 \mu m \\
\text { [mag.] }\end{array}$ & $\begin{array}{c}A_{K} \\
{[\mathrm{mag} .]}\end{array}$ \\
\hline $\mathrm{e} 1$ & 3 & $-8: 59: 06.27$ & $-47: 24: 31.0$ & $12.030 \pm 0.022$ & $10.938 \pm 0.026$ & $10.345 \pm 0.023$ & $10.016 \pm 0.025$ & $9.848 \pm 0.030$ & $9.641 \pm 0.108$ & $8.561 \pm 0.108$ & 0.36 \\
\hline $\mathrm{e} 3$ & 3 & $-8: 59: 50.53$ & $-47: 24: 31.3$ & $9.6250 \pm 0.024$ & $9.3570 \pm 0.033$ & $9.0869 \pm 0.027$ & $9.1790 \pm 0.015$ & $9.081 \pm 0.018$ & $9.046 \pm 0.028$ & $8.875 \pm 0.07$ & $\ldots$ \\
\hline
\end{tabular}

Table 15. K-S test probabilities of YSOs in RCW 38 by evolutionary class.

\begin{tabular}{rrrrrr}
\hline \hline & class 0/I & flat spectrum & class II & class III & random \\
\hline Class 0/I & $\ldots$ & 0.31 & $1.2 \times 10^{-5}$ & 0.016 & $6.9 \times 10^{-3}$ \\
Flat Spectrum & 0.31 & $\ldots$ & $1.3 \times 10^{-9}$ & 0.02 & $5.5 \times 10^{-6}$ \\
Class II & $1.2 \times 10^{-5}$ & $1.3 \times 10^{-9}$ & $\ldots$ & $2.6 \times 10^{-3}$ & $2 \times 10^{-7}$ \\
Class III & 0.016 & 0.02 & $2.6 \times 10^{-3}$ & $\ldots$ & 0.26 \\
\hline
\end{tabular}


Table 16. Properties of RCW 38 Subclusters.

\begin{tabular}{|c|c|c|c|c|c|c|c|c|c|c|c|}
\hline cluster & total & $\mathrm{C} 0 / \mathrm{I}^{\mathrm{a}} \mathrm{I}$ & $\mathrm{FS}$ & CII & CIII & Var & OB & $\mathrm{A}_{K}$ & ${ }^{\text {dered }} \alpha_{\text {irac }}$ & Protostars (\%) & Variables (\%) \\
\hline $\mathrm{NE}$ & 11 & 1 & 1 & 9 & & 1 & 1 & 0.684 & $-0.996 \pm 0$ & $18.2=$ & 9.1 \\
\hline NW & 62 & $\cdots$ & 9 & 53 & $\ldots$ & 8 & 1 & $0.608 \pm$ & $-1.078 \pm 0$ & $14.5 \pm 4.8 \%$ & $12.9 \pm 4.5 \%$ \\
\hline SW & 41 & $\ldots$ & 4 & 37 & $\ldots$ & 3 & 1 & $0.530 \pm$ & $-1.146 \pm 0.541$ & $4.9 \%$ & $7.3 \pm 4.2 \%$ \\
\hline $\mathrm{CN}$ & 255 & 15 & 39 & 154 & 47 & 24 & 6 & $0.836 \pm 0.747$ & $-0.957 \pm 1.295$ & $25.9 \pm 3.5 \%$ & $9.4 \pm 1.9 \%$ \\
\hline Dist. & 222 & 7 & 31 & 157 & 27 & 36 & 20 & $0.701 \pm 0.448$ & $-1.056 \pm 0.808$ & $19.5 \pm 3.2 \%$ & $16.2 \pm 2.7 \%$ \\
\hline
\end{tabular}

${ }^{\mathrm{a}} \mathrm{C} 0$ /I: class 0/I, FS: flat spectrum, CII: class II, CIII: class III, Var: variables, OB: OB star candidates, $\mathrm{A}_{K}$ : extinction at K-band, ${ }^{\text {dered }} \alpha_{\text {irac }}$ : slope of IRAC SED. 


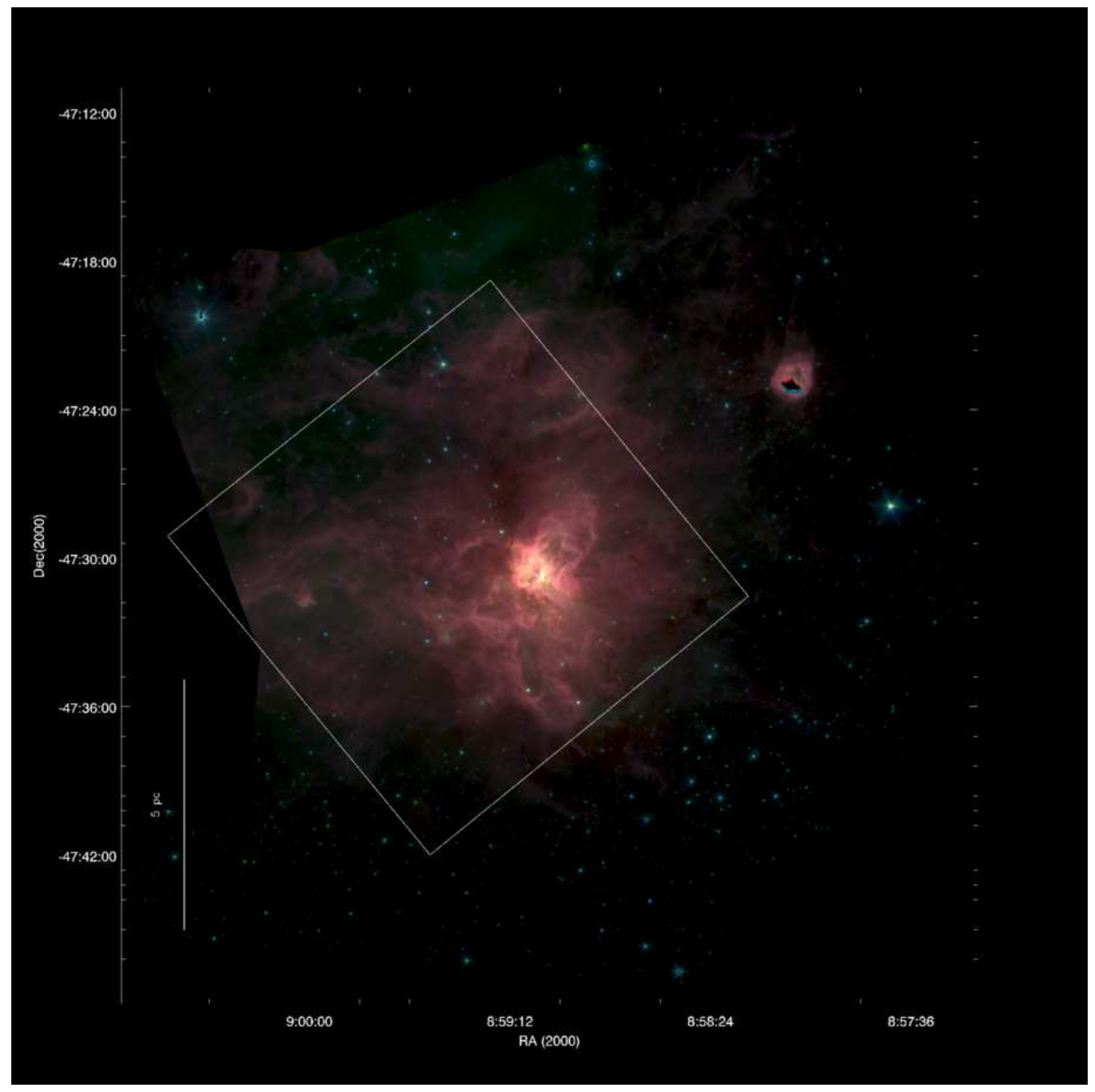

Fig. 1. - The RCW 38 region observed with IRAC on Spitzer. The plot shows a three-band false color image of the cluster, where the mosaic at each wavelength was created from the four epochs of data combined using the Montage mosaicing software. The field shows the overlap region of the four IRAC bands. Blue is $3.6 \mu \mathrm{m}$, green is $4.5 \mu \mathrm{m}$, and red is $8.0 \mu \mathrm{m}$. The reddish hue at $8.0 \mu \mathrm{m}$ is due mainly to diffuse PAH emission. Emission from shocked hydrogen is visible in green. The outline of the Chandra ACIS-I field of view is overlaid as a white square. 


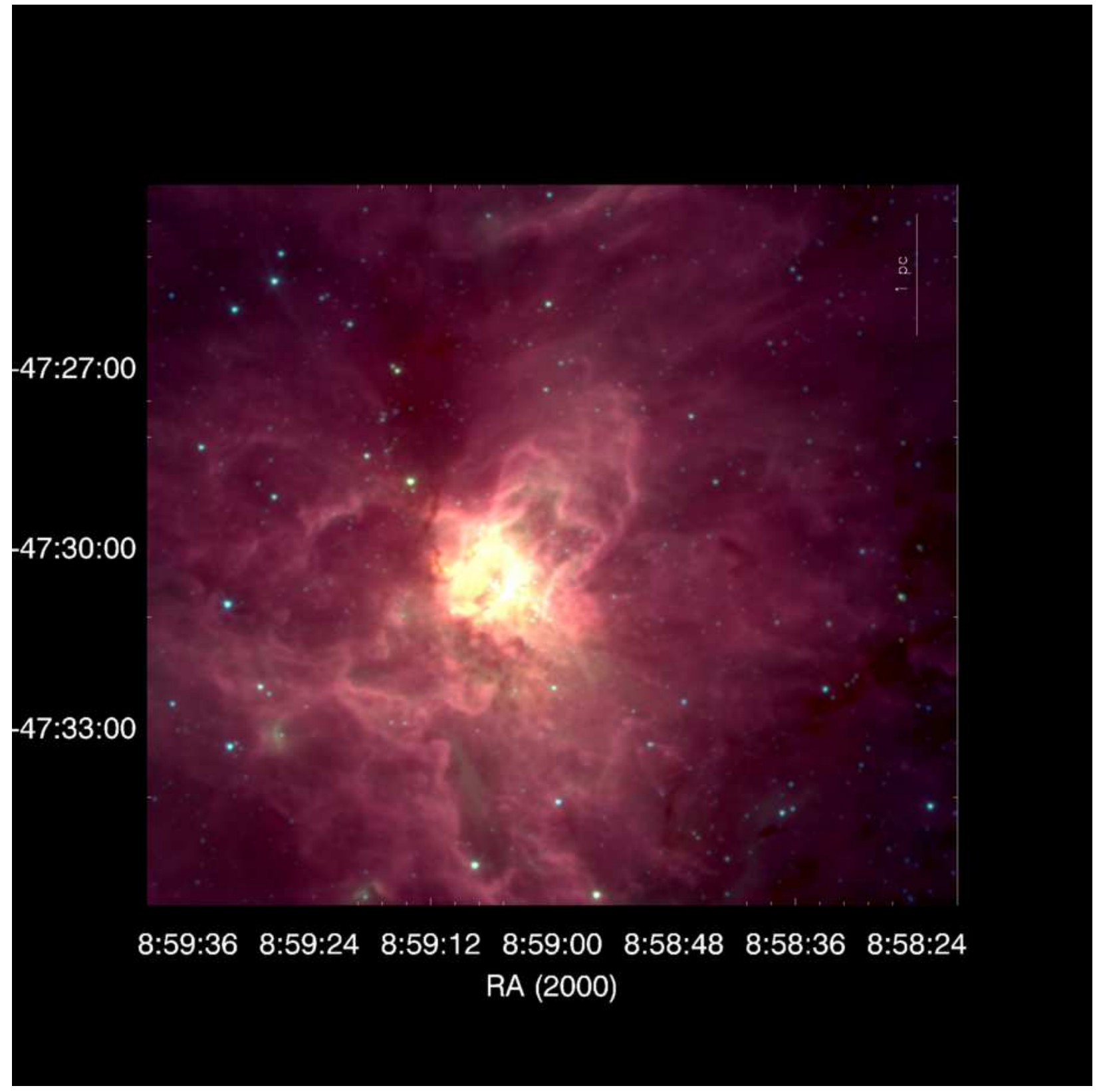

Fig. 2.- The RCW 38 central core, surrounding IRS 2. The plot shows a three-band false color image of the cluster using IRAC on Spitzer. Blue is $3.6 \mu \mathrm{m}$, green is $4.5 \mu \mathrm{m}$, and red is $8.0 \mu \mathrm{m}$. The reddish hue at $8.0 \mu \mathrm{m}$ is due mainly to diffuse PAH emission. Emission from shocked hydrogen and $B r_{\alpha}$ is visible in green. 

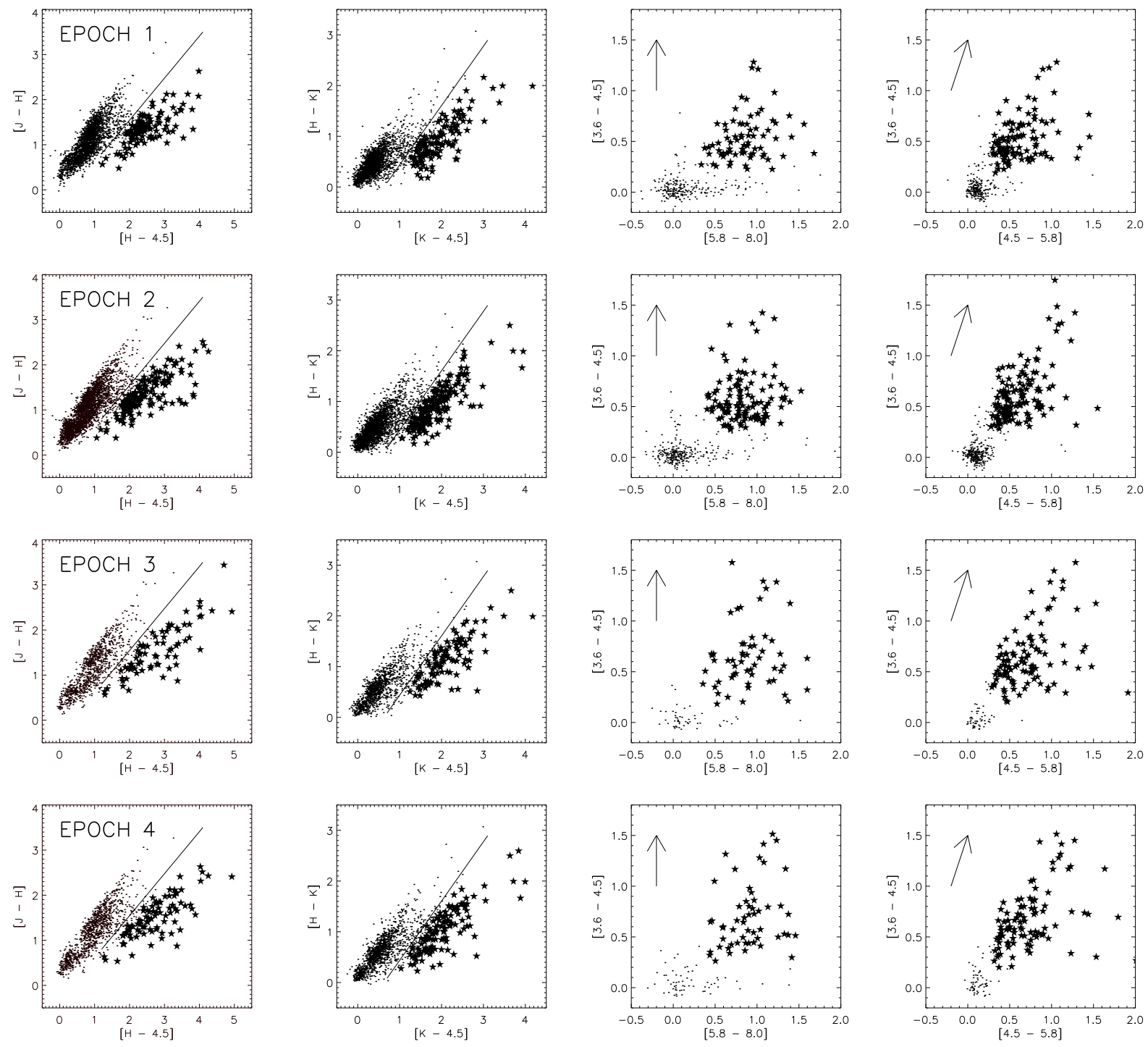

Fig. 3.- Four color-color diagrams used to identify YSOs, top to bottom: Epochs 1 - 4. Left plots: $J-H$ vs. $H-[4.5]$ diagrams. The solid line shows the reddening vector for these wavelengths; objects with $H-[4.5]$ greater than $1 \sigma$ below the reddening vector are considered to have an excess. The black dots indicate those objects classified as having no excess in this diagram, while the stars indicate those sources with an excess. Centre Left plots: $H-K$ vs. $K-[4.5]$ diagrams. Similar to the right plots, with stars indicating those sources with excess in their $K-[4.5]$ colors. Centre Right plots: IRAC color-color diagrams, [3.6] - [4.5] vs. [5.8] - [8.0]. The locus for field stars lies on the origin; the spread in the [5.8] - [8.0] colors is in part due to contamination from nebulosity and contamination from star-forming galaxies with strong PAH emission in the 5.8 and $8.0 \mu \mathrm{m}$ bands. A reddening vector of $A_{K}=5$ is shown. Right plots: IRAC color-color diagrams, [3.6] - [4.5] vs. [4.5] - [5.8]. The locus for field stars lies near the origin; the [4.5] - [5.8] color is in part due to contamination from nebulosity, such contaminants are subsequently filtered. A reddening vector of $A_{K}=5$ is shown. 


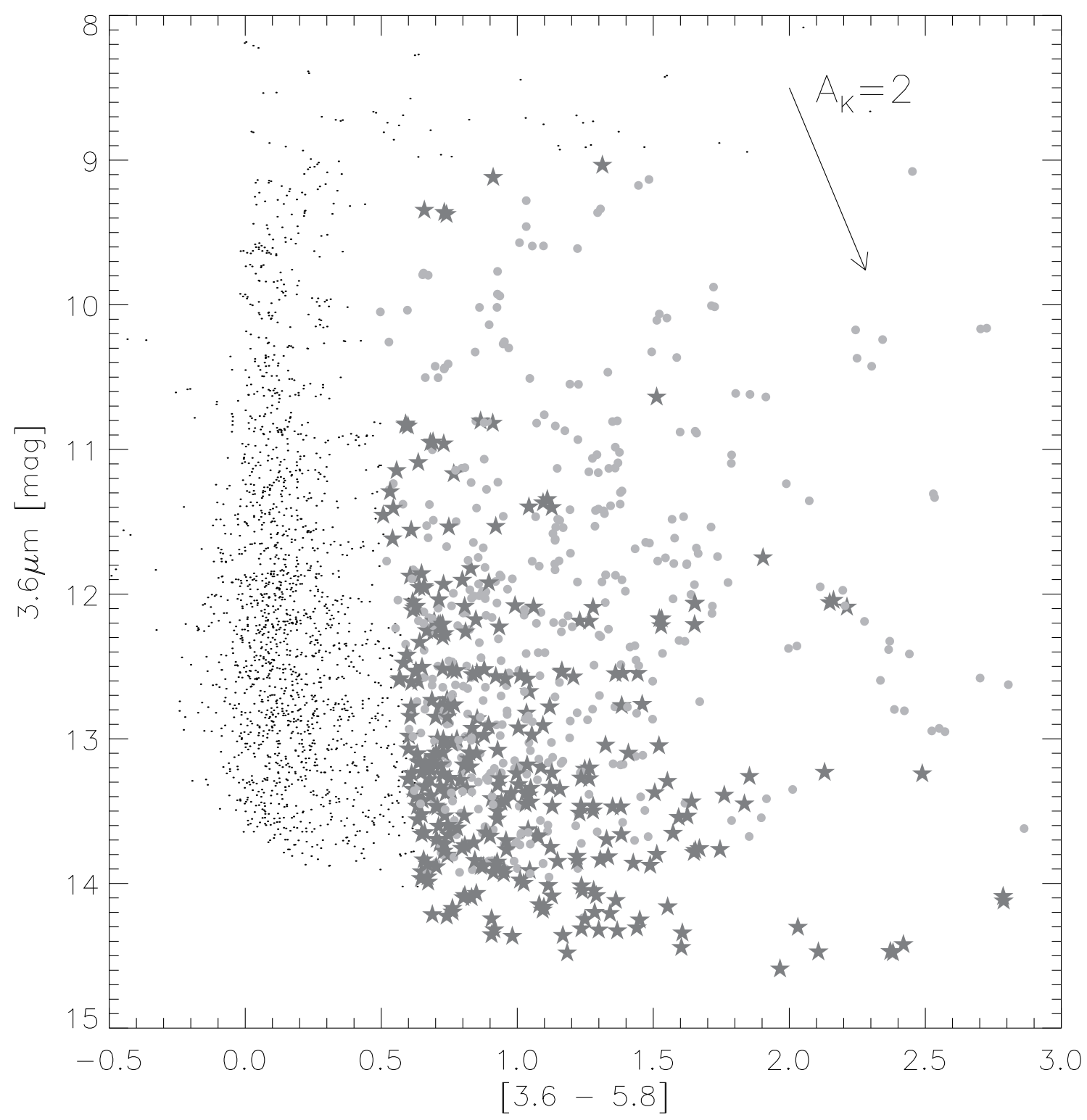

Fig. 4.- [3.6] vs. [3.6] - [5.8] color-magnitude diagram used to identify candidate YSOs. The field stars are shown as black dots, the previously identified YSOs as grey circles, and the newly selected candidates as dark grey stars. Sources with $8.0 \leq m_{3.6} \leq 14.0$ and a color more than $1 \sigma$ greater than $[3.6-5.8]>0.4$ were selected. A further 177 candidate YSOs were identified. 

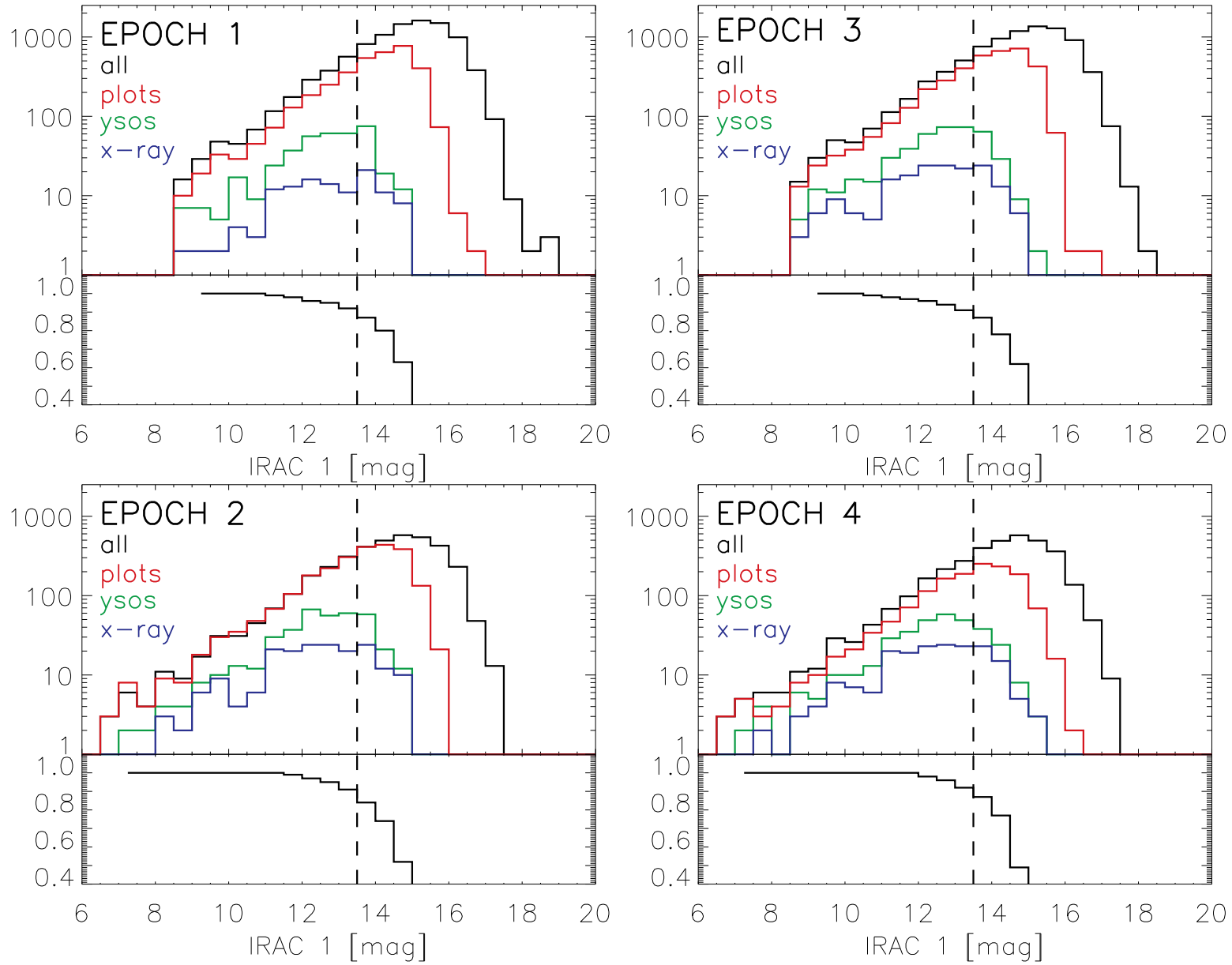

Fig. 5.- Above: Histograms of all photometry with a 3.6 $\mu m$ detection in the IRAC four band overlap region, by magnitude, as a solid black line. The red line shows those sources with photometry in the multiple bands required to place them on the color-color diagrams used to select IR excess sources. The green line shows the final YSO catalog. The blue line plots the subset of YSOs in the final catalog with a Chandra detection; note that the X-ray observations covered a smaller field than the IR observations. Below: Fraction of artificial stars recovered as a function of magnitude for the $3.6 \mu \mathrm{m}$ photometry. The dashed black line indicates $90 \%$ completeness. 

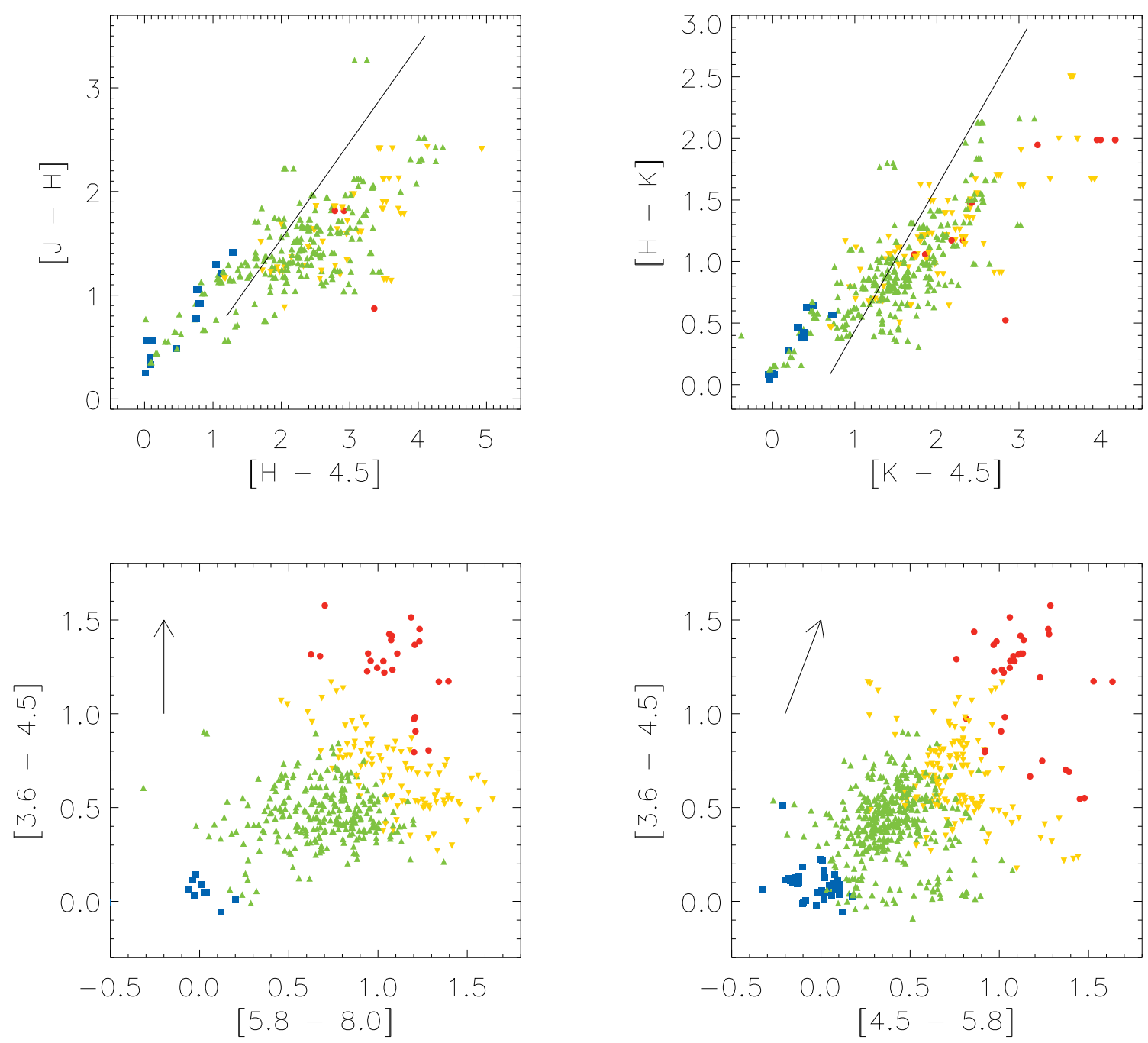

Fig. 6.- The same color-color diagrams as Fig $[3$, in this case indicating the positions of the YSOs identified in our analysis. The Class 0/I objects are marked by red circles, inverted yellow triangles mark the flat spectrum sources, green triangles mark the Class II objects. The X-ray identified Class III are shown by blue squares. On the IRAC color-color diagram some of the Class II stars appear to fall in the wrong region of the diagram for their class. An examination of the dereddened SEDs of these sources shows that their positions on the color-color diagrams have been shifted by reddening. 

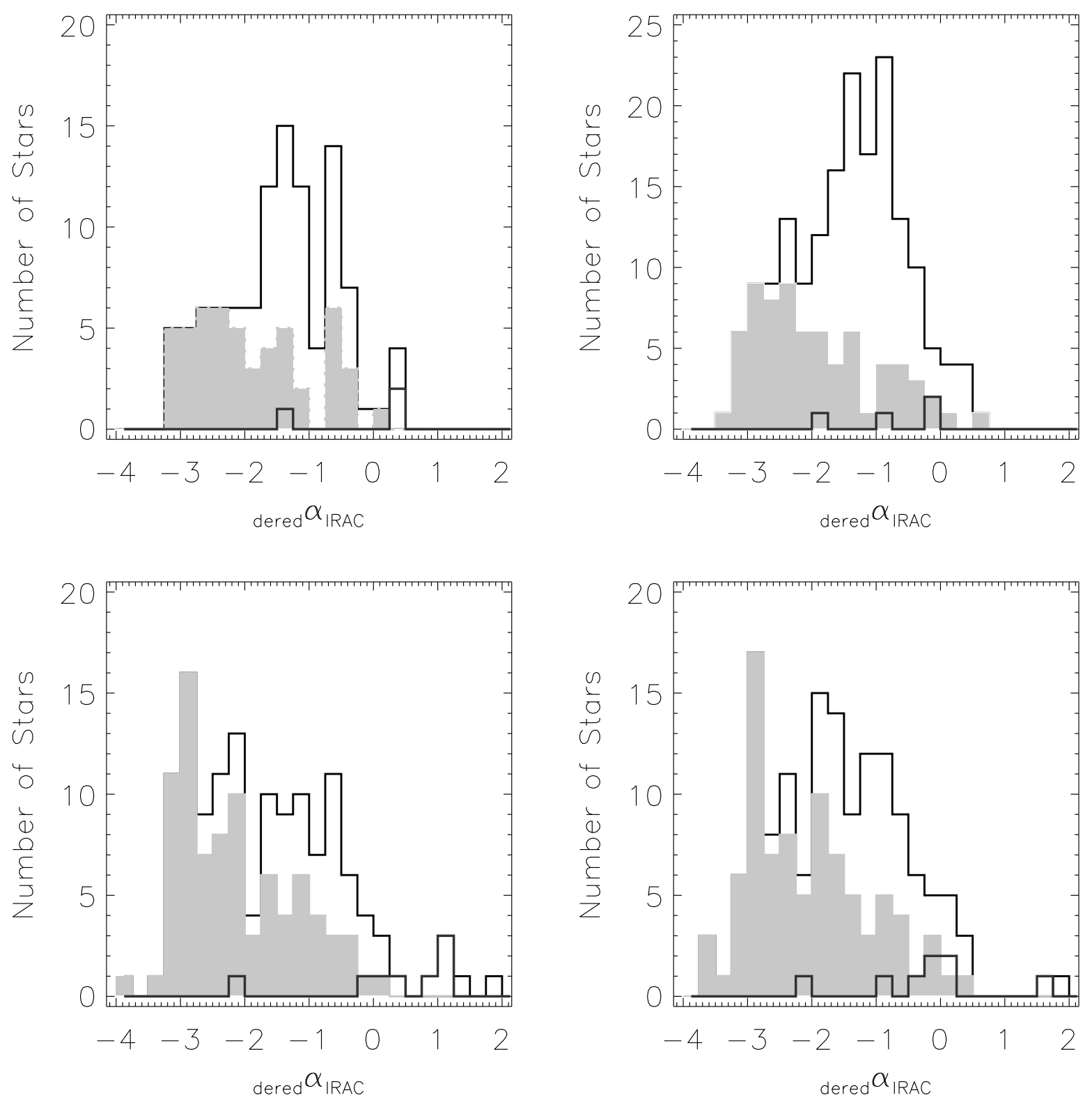

Fig. 7.- Histograms of the dereddened SED slope, dered $\alpha_{I R A C}$, of the identified cluster members of RCW 38. Upper left: The upper black histogram shows the identified YSOs in Epoch 1 with enough detections across the 2MASS and IRAC bandpasses to dereddened the photometry and to calculate ${ }_{d e r e d} \alpha_{I R A C}$. The gray filled histogram shows the subsample of the X-ray selected YSOs in Epoch 1. The lower black histogram shows the candidate variable sources. Lower left: Similarly for Epoch 2. Upper right: Similarly for the Epoch 3 YSOs. Lower right: Similarly for Epoch 4. 

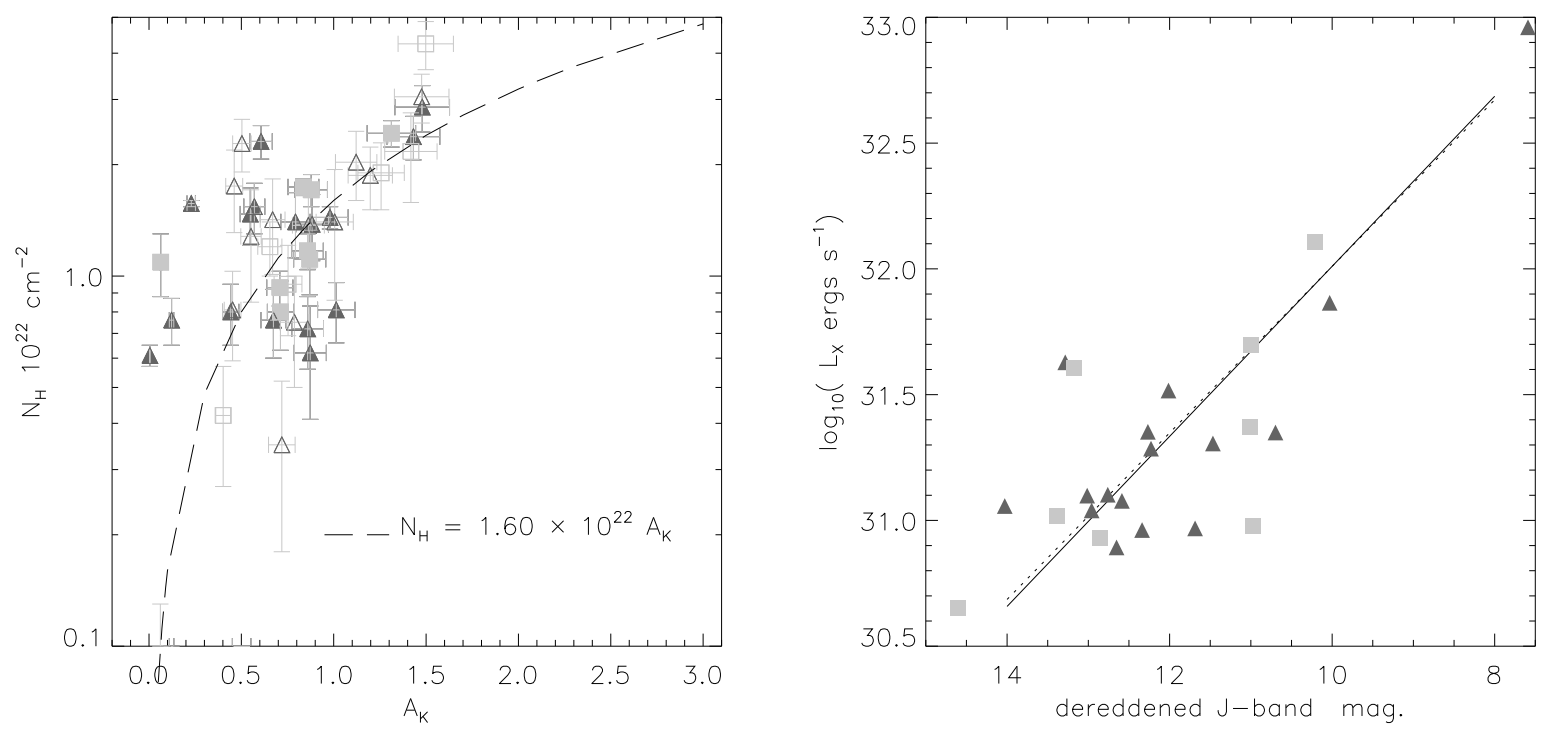

Fig. 8.- Left: The hydrogen column density, $N_{H}$, against extinction at $K$-band, $A_{K}$. The gas density was calculated from the Chandra data. The line indicates the standard $N_{H} / A_{K}$ ratio of $1.6 \times 10^{22}$, showing a statistically good fit to the data. The filled symbols are class II (mid gray triangles), and class III (light gray squares) with >100 counts; the open symbols indicate sources with $>50$ counts. Right: The X-ray luminosity, $L_{X}$, against the dereddened $J$-band magnitude (proxy for the bolometric luminosity). No difference is observed between the X-ray luminosities of the class II (black triangles) and III (grey squares) sources with $J$ magnitude, the solid line indicates the linear fit to the class II objects, the dashed line the fit to the class III. 

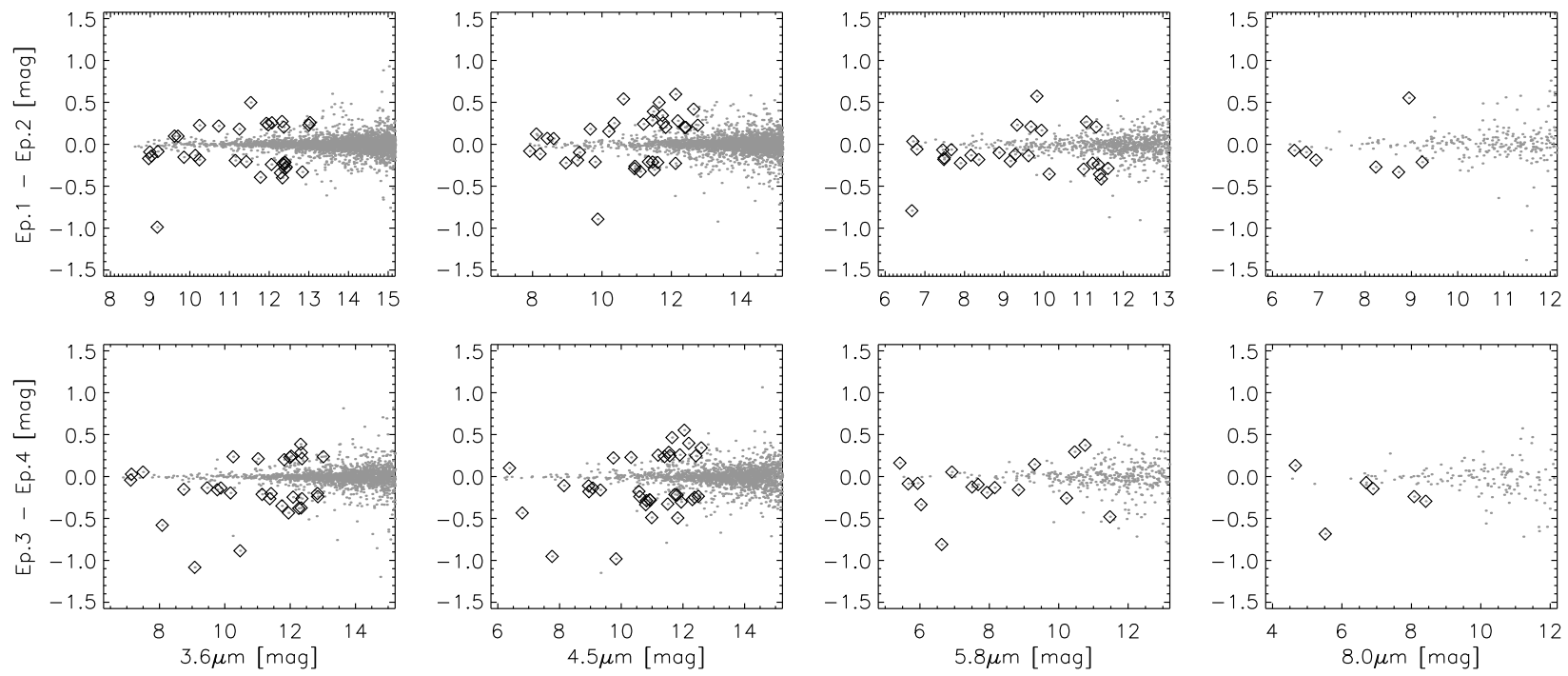

Fig. 9.- Variance with magnitude of the 72 candidate variables in RCW 38 over the four IRAC bandpasses. In the identification of variable candidates, only the comparison of photometry from [Ep.1 - Ep.2] and [Ep.3 - Ep.4] was considered as Epochs $1 \& 2$ are the larger fields surrounding the core and have longer integration times, and Epochs $3 \& 4$ are the exposures of RCW 38 core. Sources were selected as variables if they exhibited a magnitude difference in any band greater than $3 \sigma$. Only sources with uncertainties $<0.1$ mag were considered. 

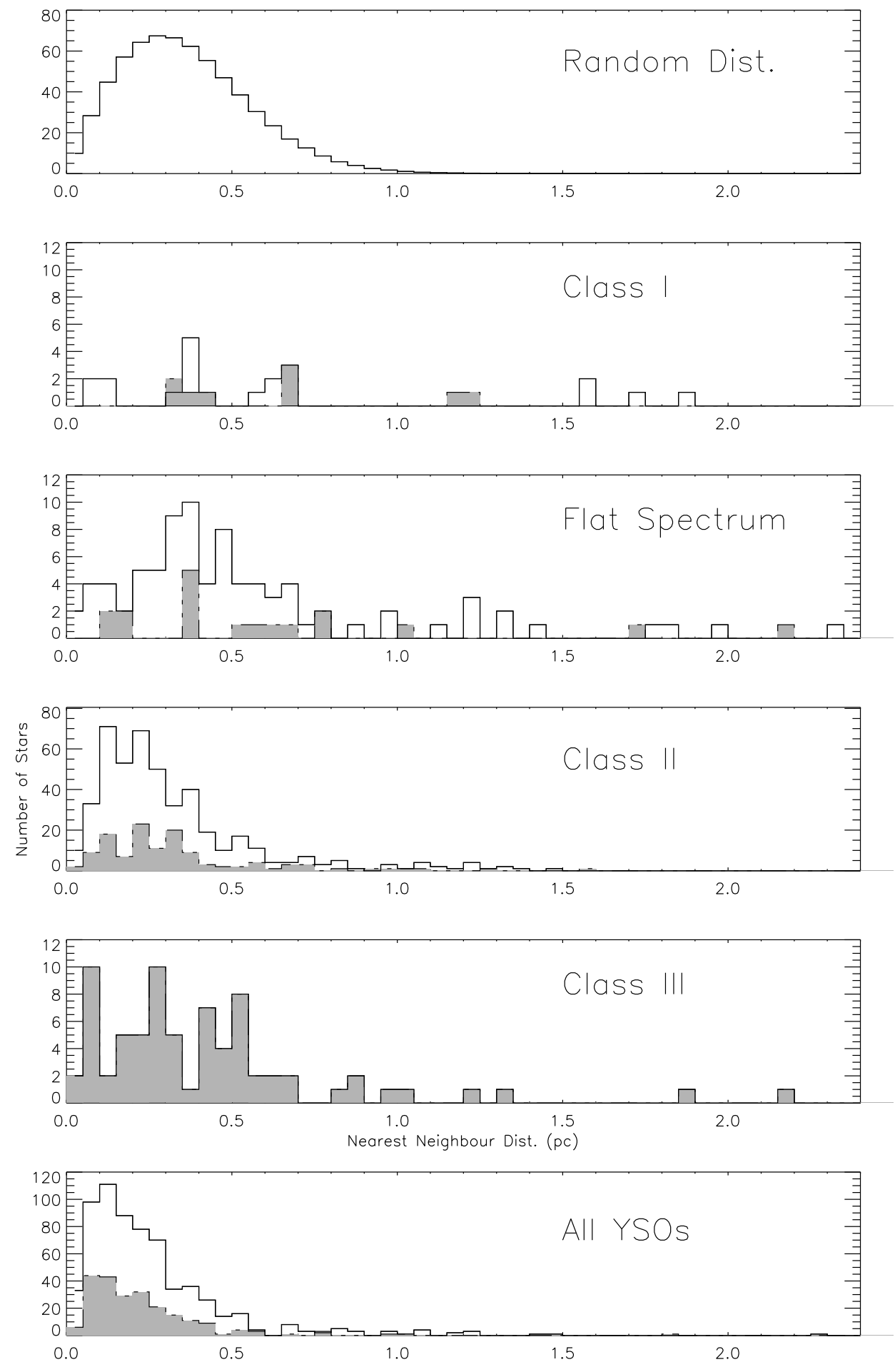

Fig. 10.- Differential distribution of nearest neighbor distances by class for all the identified YSOs, shown by the solid black line; the shaded region gives the differential distribution of nearest neighbor distances by class for the X-ray selected sample of all YSOs. The stellar sources are more densely clustered than the protostellar objects. The top panel gives the nearest neighbor distances for a random distribution. 

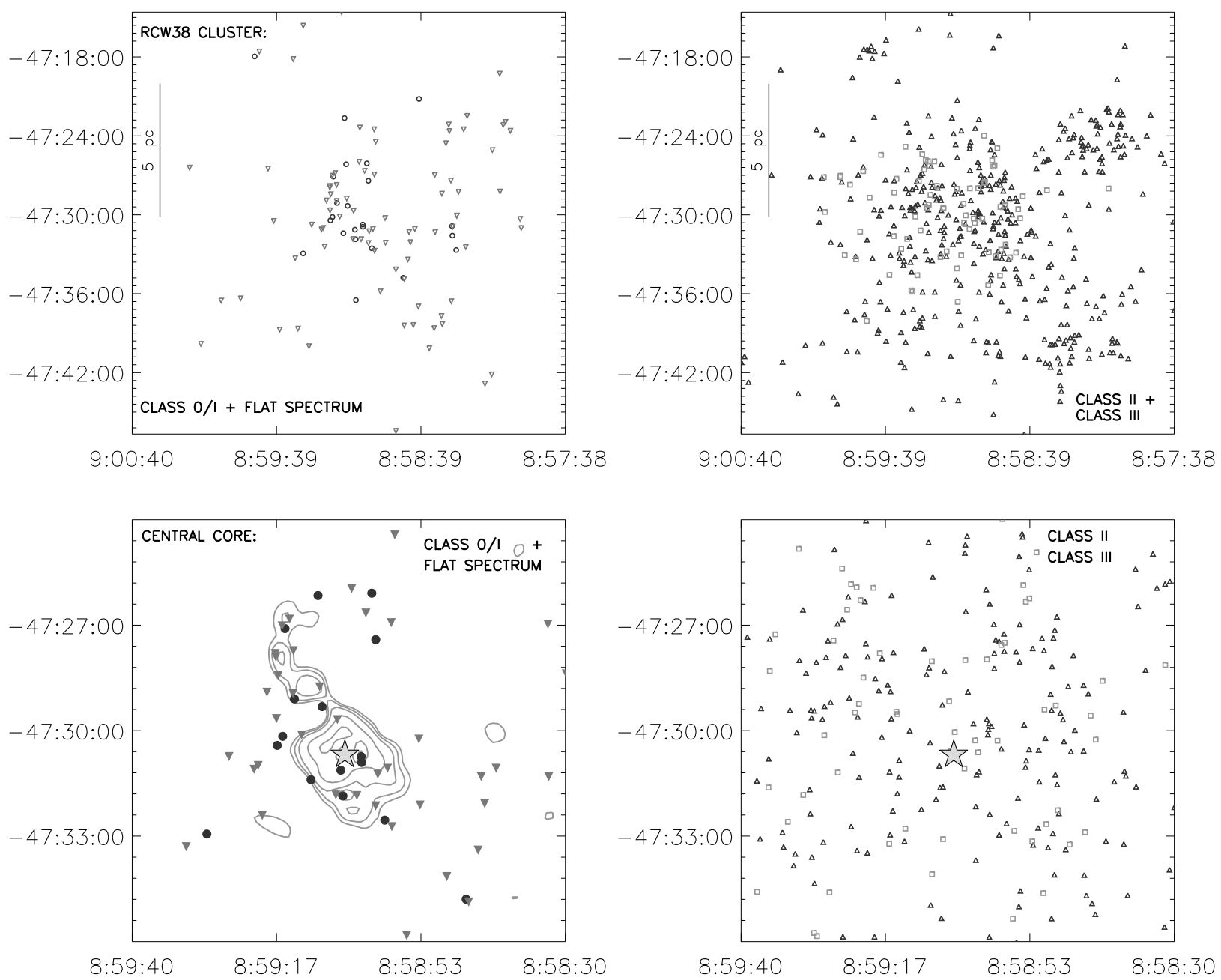

Fig. 11. - Spatial distribution of the various classes of YSOs in the RCW 38 cluster. Above Left: Class 0/I and flat spectrum sources, shown by circles and inverted triangles, respectively. Above Right: Class II objects and class III sources, shown by triangles and squares, respectively. Evidence of subclustering can be seen from the distribution of CII/III sources and the distribution of protostellar objects. Note that the class III distribution is delimited by the Chandra field of view, which is smaller than that of IRAC. Expanded view of the YSOs in the centre of the RCW 38 cluster, in a $\sim 0.2$ deg diameter of the central $\mathrm{O}$ star binary. Below left: Class 0/I and flat spectrum sources, shown by circles and inverted triangles, respectively. The Vigil (2004) SIMBA $1.2 \mathrm{~mm}$ map of the region is shown in contours, with contour levels of $[58 \%, 59 \%, 61 \%, 70 \%, 85 \%, 95 \%]$. Below right: Class II objects, shown by triangles, and class III sources, shown by squares. The stellar members appear to be evenly distributed, while the protostellar objects form an arc roughly centered on the $\mathrm{O}$ star binary, IRS 2. The protostars appear coincident with the denser dust. 


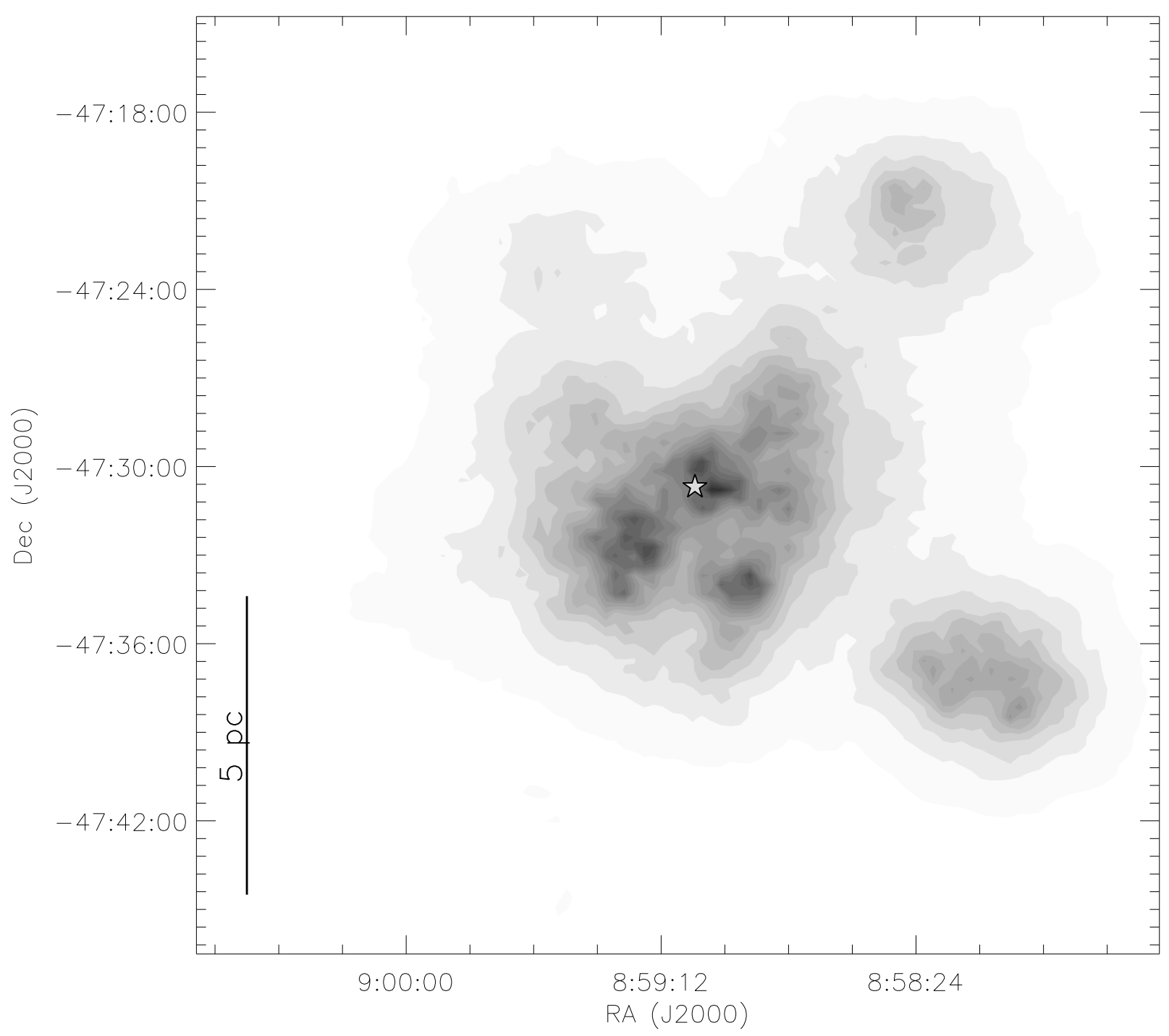

Fig. 12. - Surface density map of the YSOs in the RCW 38 cluster. The stellar surface density was calculated with a grid size of $100 \times 100$ using $N=18$, the distance to the 18 th nearest neighbor, to smooth out smaller scale structure. The density contours range linearly from $0 / p c^{2}$ (white) to $30 / p c^{2}$ (black). The surface density map also indicates the presence of two subclusters to the NW and SW, and a possible third subcluster to the NE, of the central core, which itself shows three density peaks. The position of IRS 2 is marked by a star. 


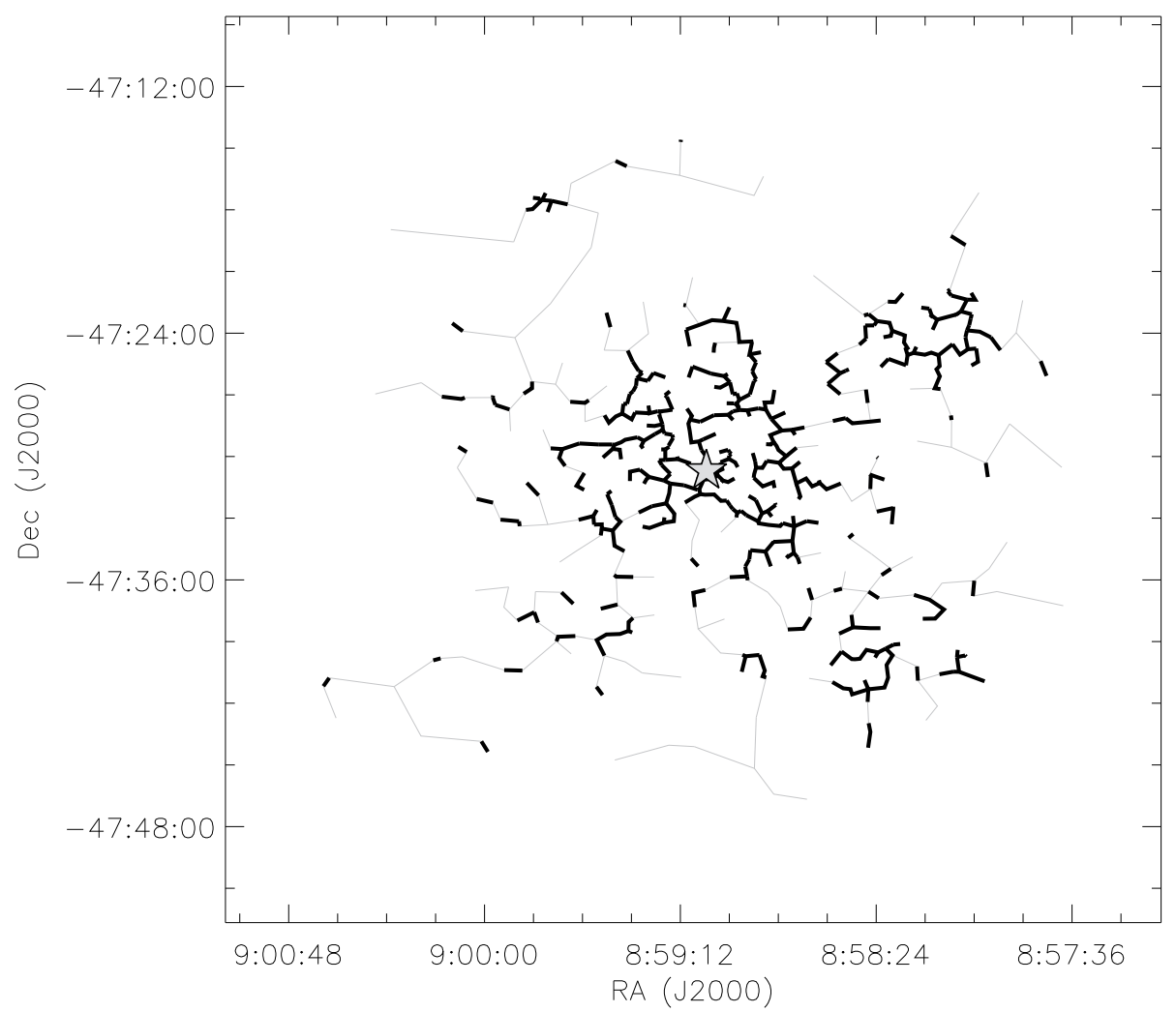

Fig. 13. - Minimum spanning tree (MST) of the YSOs detected in the RCW 38 cluster. The vertices represent the positions of the YSOs. The black branches are those with a length less than the characteristic branch length of 50", c.f. Fig. 14. The grey branches have lengths greater than 50". Those YSOs connected by branch lengths shorter than the characteristic length can be considered to form a cluster. The MST indicates that the RCW 38 cluster can be subdivided into the central core, focused on the O-star binary, and two separate subclusters to the NW and SW. There is also a small grouping of eight stars to the NE, which may be a developing subcluster. The position of IRS 2 is marked by a star. 


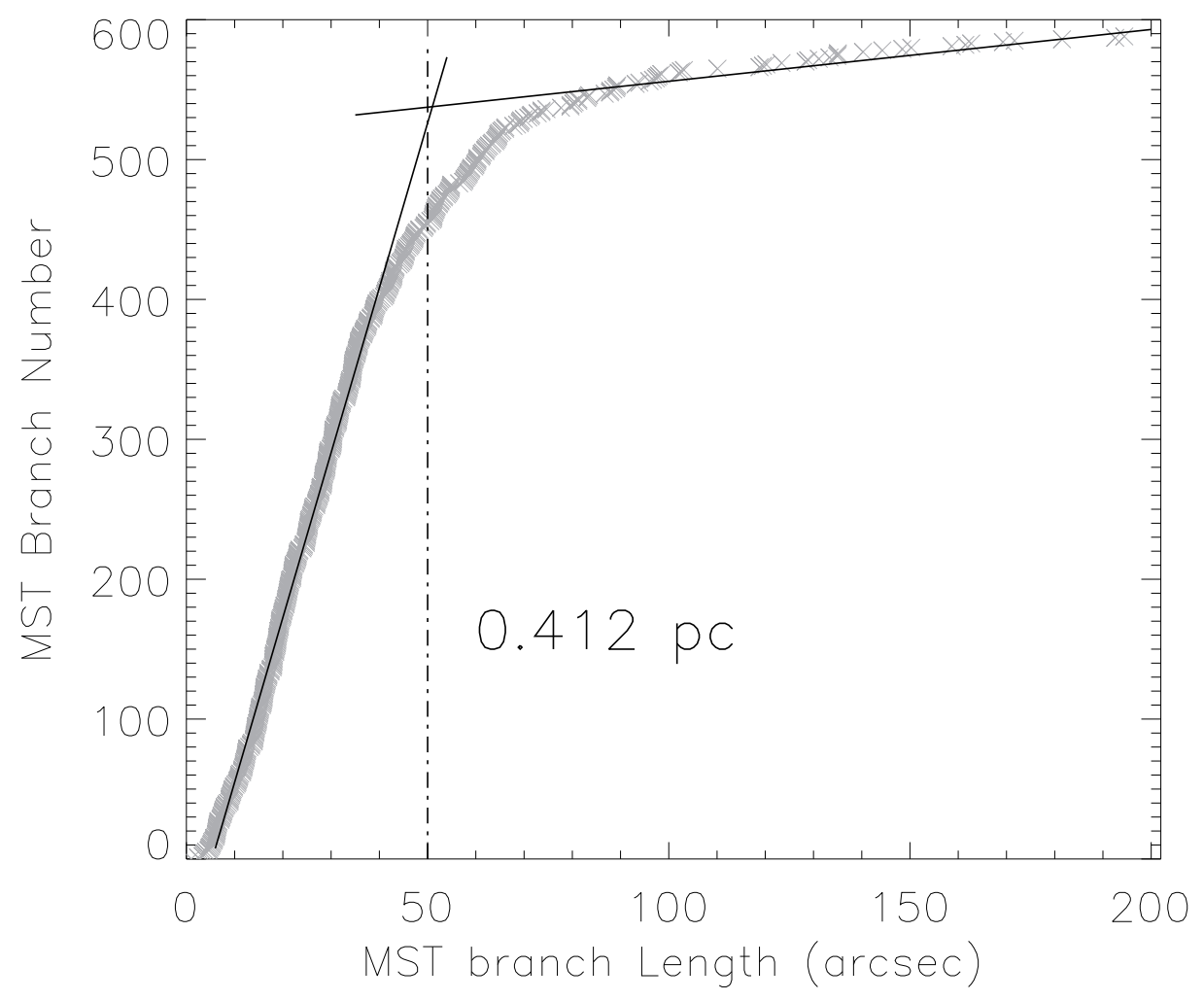

Fig. 14. - Distribution of branch lengths for the minimum spanning tree of the RCW 38 YSOs. The distribution was fitted in two linear regimes. The characteristic branch length for the cluster was found to be 50 " or $0.412 \mathrm{pc}$. 


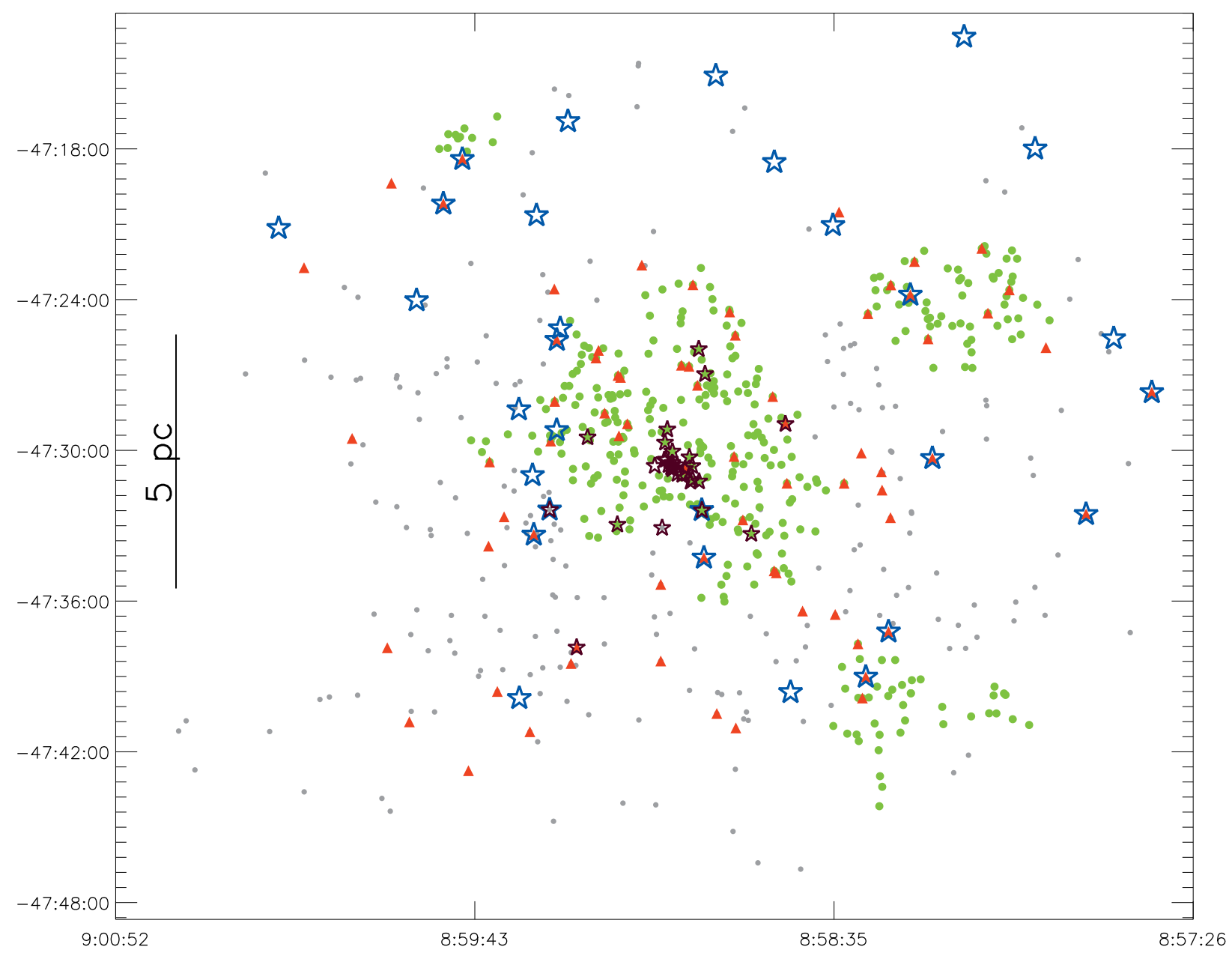

Fig. 15. - Spatial distribution of the identified YSOs in RCW 38 showing the positions of the $\mathrm{O}$ star and variable candidates. The dots indicate the young stars identified via IR excess emission and X-ray detection; clustered YSOs are shown by green dots, distributed YSOs in gray. The triangles show the positions of the variable objects, while the blue open stars show the positions of the candidate $\mathrm{O}$ stars in the field. The black open stars show the positions of the candidate OB stars identified in Wolk et al. (2006). Both the variable and O star candidates are distributed throughout the extended cluster, with candidates of both types appearing in the subclusters. 